\title{
Disgust in specific phobias : a dirt road to anxiety disorders
}

Citation for published version (APA):

van Overveld, W. J. M. (2008). Disgust in specific phobias : a dirt road to anxiety disorders. [Doctoral Thesis, Maastricht University]. Datawyse / Universitaire Pers Maastricht.

https://doi.org/10.26481/dis.20081024wo

Document status and date:

Published: 01/01/2008

DOI:

10.26481/dis.20081024wo

Document Version:

Publisher's PDF, also known as Version of record

\section{Please check the document version of this publication:}

- A submitted manuscript is the version of the article upon submission and before peer-review. There can be important differences between the submitted version and the official published version of record.

People interested in the research are advised to contact the author for the final version of the publication, or visit the DOI to the publisher's website.

- The final author version and the galley proof are versions of the publication after peer review.

- The final published version features the final layout of the paper including the volume, issue and page numbers.

Link to publication

\footnotetext{
General rights rights.

- You may freely distribute the URL identifying the publication in the public portal. please follow below link for the End User Agreement:

www.umlib.nl/taverne-license

Take down policy

If you believe that this document breaches copyright please contact us at:

repository@maastrichtuniversity.nl

providing details and we will investigate your claim.
}

Copyright and moral rights for the publications made accessible in the public portal are retained by the authors and/or other copyright owners and it is a condition of accessing publications that users recognise and abide by the legal requirements associated with these

- Users may download and print one copy of any publication from the public portal for the purpose of private study or research.

- You may not further distribute the material or use it for any profit-making activity or commercial gain

If the publication is distributed under the terms of Article $25 \mathrm{fa}$ of the Dutch Copyright Act, indicated by the "Taverne" license above, 


\section{Disgust in Specific Phobias}

A Dirt Road to Anxiety Disorders 
(c) copyright M. van Overveld, Maastricht 2008 ISBN 9789052787671

Druk: Datawyse I Universitaire Pers Maastricht Omslagontwerp: Tristan Bronkhorst

Een digitale versie van deze uitgave is te vinden op: http://dissertaties.ub.unimaas.nl 


\title{
Disgust in Specific Phobias
}

A Dirt Road to Anxiety Disorders

\author{
Proefschrift \\ ter verkrijging van de graad van doctor aan de Universiteit Maastricht, \\ op gezag van de Rector Magnificus, Prof. mr. G.P.M.F. Mols, \\ volgens het besluit van het College der Decanen, \\ in het openbaar te verdedigen op vrijdag 24 oktober 2008 om 16.00 uur. \\ door
}

Waltherus Johannes Maria van Overveld

$\underbrace{\int_{1}^{\top}}_{\substack{\text { UNIVERSTAIRE } \\ \text { PESS MASTRICHT }}}$ 


\section{Promotores}

Prof. dr. P. J. de Jong (Rijksuniversiteit Groningen)

Prof. dr. M. L. Peters

\section{Beoordelingscommissie}

Prof. dr. A. Arntz (voorzitter)

Prof. dr J. van Lankveld

Prof. dr. P. Muris (Erasmus Universiteit Rotterdam),

Dr. C. Nederkoorn

Prof. dr. B. Olatunji (Vanderbilt University USA) 


\section{Contents}

Chapter 1 A new perspective in psychopathology: The gooy, the bah, and the yucky 9

Chapter 2 The Disgust Scale-R: A valid and reliable index to investigate separate 21 disgust domains?

Chapter 3 Disgust propensity and disgust sensitivity: Separate constructs? 33

Chapter 4 The Disgust Propensity and Sensitivity Scale - Revised: Its predictive 45 value for avoidance behavior and physiological responsivity.

Chapter 5 Differential startle modulation during fear and disgust: Influence of individual differences in disgust propensity and sensitivity.

Chapter 6 Disgust propensity and disgust sensitivity are differentially associated with psychopathological symptoms.

Chapter 7 An internet-based study on the relation between disgust sensitivity and emetophobia.

Chapter 8 Digestive and cardiovascular responses to core and animal-reminder disgust.

Chapter 9 Differential UCS expectancy bias in spider fearful individuals: Evidence towards an association between spiders and disgust-relevant outcomes.

Chapter 10 Disgust and fear related UCS-expectancy bias in blood fearful individuals

Chapter 11 Contamination and harm relevant UCS-expectancy bias in spider phobic individuals: Influence of treatment and its relationship with the return of fear

Chapter 12 Discussing the dirt road

Summary 
'Well I ain't goin' down, that dirt road by myself' Howlin' Wolf 


\section{Chapter 1}

\section{A new perspective in psychopathology: The gooy, the bah, and the yucky}




\section{CHAPTER 1}

Having reached the first page of this dissertation, you may consider yourself a lucky person. You have either proven to: a) be in perfect (mental) health, or b) be able to conquer your greatest fears. Despite the horrific pictures on its outer sleeve, you did not fling this bundle of papers out of the window, nor did you run away in extreme terror, and although several inner sensations may have overcome you upon looking at the sleeve, you apparently did not engage in behavioral withdrawal of this dissertation.

This dissertation will focus mostly on the sensations during your first look at the outer sleeve. The various objects on the sleeve are generally associated with fear. Yet, you may have noticed another emotion, namely, the emotion of disgust, when you gazed upon the spider or the zombies. But what is disgust? And could it be involved in the desire to remove the beforementioned outer sleeve from the dissertation, in other terms, active (and possibly phobic) avoidance behavior for the various disgust-related stimuli?

Initially, the emotion of disgust was largely neglected in emotion research. With regard to the role of disgust in psychopathology, some authors even cynically dubbed it 'the forgotten emotion of psychiatry' (Phillips, Senior, Fahy, \& David, 1998) and wondered whether researchers possibly demonstrated an elaborate aversion towards the study of disgust (Olatunji \& Sawchuk, 2005). Fortunately, a steady increase in the number of papers on the relation between disgust and psychopathological conditions has been noted in the past two decades, and have successfully proven its worth to clinical research by linking disgust to various conditions, like spider phobia (Matchett \& Davey, 1991), blood phobia (Page, 1994), obsessive compulsive disorders (Thorpe, Patel, \& Simonds, 2003), eating disorders (Troop, Murphy, Bramon, \& Treasure, 2000) and sexual dysfunctions (de Jong, van Overveld, Weijmar-Schultz, Peters, \& Buwalda, in press). So, although the emotion of disgust certainly remains an understudied phenomenon in contrast to other negative emotions like fear or anger (see for example Figure 1. for a Psychinfo search on the number of published articles on these three negative emotions in the past five decades using search terms 'anger', 'disgust', and 'fear'), disgust does appear to have arrived as a topic of interest in clinical research (McNally, 2002).

As indicated, there has been a steady increase in research on the function of disgust and its relationship to a variety of psychopathological complaints in the past decade. This first chapter will summarize the main findings and serve to define the concept of disgust, and how it is associated with several forms of psychopathology. Furthermore, it will be outlined what scientific gaps currently still remain, and which will be investigated in this dissertation. 


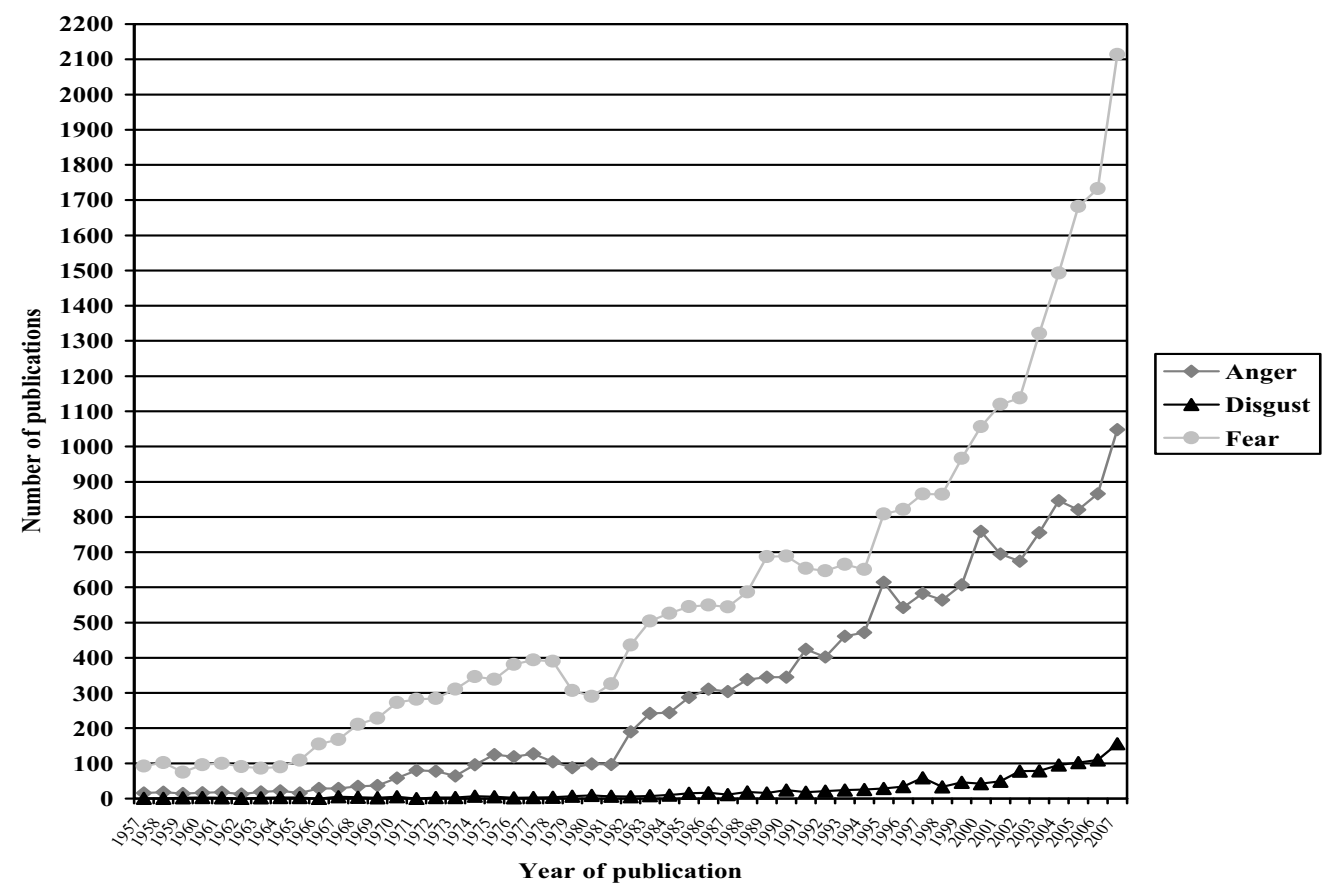

Figure 1. Results of a Psychinfo search on the number of published articles on anger, disgust, and fear in the past five decades (1957 to 2007).

\section{Definition of disgust}

One of the most popular definitions of disgust used in the literature stems from Darwin (1865), and defines disgust as: '..refers to something revolting, primarily in relation to the sense of taste, as actually perceived or vividly imagined; and secondarily to anything which causes a similar feeling, through the sense of smell, touch and even of eyesight' (Rozin, Haidt \& McCauley, 2000). Disgust is usually experienced at the threat of intimate contact with certain substances, such as faeces, sweat or other waste products of the human body (Angyal, 1941). The more intimate the contact, the more intense the disgust response will be (Fallon \& Rozin, 1983; Fessler \& Haley, 2006). Consequently, the most threatening prospect would be oral incorporation. Disgust would thus function as a defense mechanism, shielding humans from pollution by disgusting objects (Olatunji \& McKay, 2007).

Further, disgust is believed to be qualitatively different from similar concepts like distaste as the motivation for food rejection in disgust is not merely motivated by sensory characteristics. Two components appear crucial for an object in order to qualify as a disgusting stimulus (Rozin, Haidt, \& McCauley, 2000). Firstly, the nature of the stimulus itself is important. For example, most people would render a cockroach not fit for consumption, despite its nutritional value. Secondly, the stimulus must have the capability to contaminate. For example, Rozin et al. (1999) dipped a cockroach in someone's drink right before the eyes of the participants. 


\section{CHAPTER 1}

Following the removal of the cockroach, most participants were less willing to take another sip of this beverage or even of a freshly poured but unspoiled glass of the same beverage. Presumably, ingestion of offensive objects transmits the offensiveness of the object onto the person ingesting (Olatunji \& McKay, 2007).

\section{Disgust propensity and specific phobias}

Like any emotion, disgust is proposed to have both a 'state' and 'trait' component. 'State' disgust can be defined as the directly experienced disgust in the presence of a disgusting stimulus, which can be both external (e.g., taking a sip of a cup of coffee in which a fly is floating) and internal (e.g., thinking of that time you took a sip of a cup of coffee in which a fly was floating). For example, it is the emotion that may have befallen you at the first gaze of this dissertation's outer sleeve. 'Trait' disgust refers to a relatively stable personality trait that determines how readily people respond with the emotion of disgust to different situations. This is called disgust propensity or disgust disposition. A certain level of disgust propensity characterizes each person, and individual variation in this trait explains why some will experience extreme disgust upon looking at the sleeve, whereas others may probably wonder why the author did not use more potent stimuli.

Recent studies have implicated that disgust propensity could be related to the etiology of small animal phobias; particularly for small animals for which feelings of fear should not really be necessary such as spiders. Spiders, and most certainly the species that are known in Western Europe, usually do not attack people. Moreover, only a small fraction of all spider species actually pose a realistic threat to humans (Renner, 1990). Therefore, in search of an explanation for such fear of small, harmless animals the disease-avoidance model was postulated (Matchett \& Davey, 1991). According to this model, people try to defend themselves from physical contact with such animals (e.g., spiders, rats), because of their (assumed) potential to spread diseases. Disgust could function as a motivator, because by being disgusted of certain animals, one will actively try to avoid them. A strong disgust propensity could then even be evolutionary functional, as it may shield people from the contaminating pathogens that these animals potentially carry (Matchett \& Davey, 1991). However, it may similarly be argued that the disease avoidance model reflects a post hoc rationalization for an ill-understood phenomenon rather than actual fact, given that most spiders are not usually associated with the spread of diseases (McNally, 2002). So, although disgust may drive avoidance behavior for spiders, it may be that this tendency is not necessarily an evolved adaptation that promoted Darwinian fitness amongst our ancestors. Nevertheless, a series of research findings confirmed that disgust propensity was indeed heightened in spider phobic individuals (de Jong \& Merckelbach, 1998; de Jong et al., 2002; Mulkens et al., 1996; Sawchuk et al., 2000). Furthermore, heightened (general) disgust propensity appeared paralleled by increased disgust responding in the sense that spiders evoked disgust-related physiological reactivity in spider phobic individuals (i.e., disgust-specific facial EMG; de Jong, Peters, \& Vanderhallen, 2002). Furthermore, disgust propensity was closely associated with behavioral avoidance of spiders 
(Olatunji \& Deacon, 2008) as well as state disgust (Woody \& Tolin, 2002). Moreover, spider phobic individuals reported spiders to be the most 'disgusting' object known to them (Thorpe \& Salkovskis, 1998) and this disgust response appeared enhanced specifically for spiders (Olatunji, 2006). Moreover, state disgust even proved a better predictor than fear for the tendency to avoid spiders (Woody, McLean, \& Klassen, 2005).

Further, inflated levels of disgust propensity are not just observed in individuals with a phobia for small animals (e.g., spiders, snakes) but also in blood phobic individuals (Sawchuk et al., 2002). The finding that disgust propensity may also be inflated in blood phobic individuals has been repeatedly confirmed (Page, 1994; 2003; Schienle et al., 2005, Olatunji et al., 2004). These findings are further substantiated by the observations that upon confrontation with phobic material, blood phobic individuals respond primarily with the emotion of disgust (Tolin, Lohr, Sawchuk, \& Lee, 1997) and that disgust-specific facial EMG becomes activated more pronounced in blood fearful individuals compared to low fearful individuals (Schienle, Stark, \& Vaitl, 2001).

\section{Disgust propensity and other psychopathological conditions}

Apart from the involvement of disgust propensity in specific phobias, there have been indications that disgust propensity contributes significantly to other mental disorders as well, such as obsessive-compulsive disorders (OCD). OCD patients frequently report obsessive thoughts regarding dirt and contamination. Accordingly, OCD clients with strong contamination fears and washing compulsions displayed higher levels of disgust propensity compared to OCD clients with other fears, or patients with other anxiety disorders and non-anxious persons (Woody \& Tolin, 2002). In non-clinical volunteers, disgust propensity was shown to be the best predictor of control and washing behaviors (Mancini, Gragnani \& D'Olimpio, 2001; Olatunji, Sawchuk, Lohr \& de Jong, 2004). Moreover, disgust propensity was significantly associated with behavioral avoidance tendencies on a series of contamination-related behavioral avoidance tasks, involving exposure to a used comb, a cookie on the floor and a bedpan filled with toilet water (Deacon \& Olatunji, 2007). Hence, the possibility arises that disgust propensity is a vulnerability factor in the development of OCD.

Another group of disorders with which disgust propensity can be related are eating disorders. Here, it could be that patients are disgusted of food-items or their own body (Power \& Dalgleish, 1997). Research shows a strong correlation between symptoms of eating disorders and disgust propensity (Troop, Murphy, Bramon \& Treasure, 2000). Patients with eating disorders displayed significantly enhanced levels of disgust propensity. Heightened disgust propensity was most pronounced on subscales relating to disgust of food, body and bodily products (Davey, Buckland, Tantow \& Dallos, 1998). The notion that disgust may be of importance in eating disorders is further underlined by the finding that disgust propensity was particularly lower on subscales concerning one's own body and bodily products in people that had been treated successfully for eating disorders compared to (untreated) patients with an eating disorder (Troop, Treasure \& Serpell, 2002). Additionally, recent findings linked disgust 


\section{CHAPTER 1}

for high-energy foods and overweight body shapes with dieting concerns (Griffiths \& Troop, 2006).

Disgust propensity may further contribute to emetophobia (i.e., fear of vomiting). Not only is vomit is an universally accepted disgust stimulus, vomiting is associated with the expulsion of hazardous pathogens from the body. Thus, it seems likely that disgust propensity could be involved in the etiology of emetophobic complaints. So far, no studies have been conducted to determine whether such a correlation exists between disgust propensity and emetophobic complaints.

\section{Core and animal-reminder disgust}

Initially, disgust was defined in terms of food rejection tendencies. Disgust was defined as the reaction that occurs at the prospect of consuming a disgusting stimulus (Rozin \& Fallon, 1987). This type of disgust is labeled core disgust (Power \& Dalgleish, 1997). However, people are not only disgusted at the threat of consuming something aversive (Miller, 1997). For example, people have displayed disgust at the sight of severed corpses and body limbs scattered over a battlefield (Achard, 2002), for cannibalism (Montaigne, 1995), or even for more abstract affairs like the meaninglessness of our existence (Sartre, 1976) or even old age (Gilleard, 2007). Moreover, cultural influences are presumed to exert a great influence on what is deemed to be disgusting or acceptable (e.g., cannibalism) or even a source of amusement (e.g., throwing people in front of lions in ancient Roman arenas) (McNally, 2002). In accordance with these observations, Rozin et al. (1999) stated in their two-stage model of disgust that because of (cultural) evolution, disgust responses might have extended towards multiple domains, such as animal-reminder disgust, interpersonal disgust, and sociomoral disgust (Rozin et al., 1999).

Animal-reminder (A-R) disgust can be defined as the response that occurs when confronted with stimuli that emphasize the similarities between humans and animals (Haidt, McCauley, \& Rozin, 1994). These similarities remind people of the fact that they too are of animal origin, a thought that most people consider unacceptable (Rozin et al., 2000). Man wants to rise above the animal kingdom, so generally, humans do not want to be reminded that they too are mortal creatures of flesh and blood, committing beastly acts, such as sex or excretion. Consistent with the two-stage model of disgust, recent investigations have indicated that these separate domains of disgust elicitors could be reliably distinguished as separate constructs (Olatunji et al., 2007).

Interpersonal disgust is the disgust at the prospect of (in)direct contact or intimacy with certain people. The purpose of experiencing people as disgusting is to be able to select persons as possible partners for intimate social contact (such as the sharing of food, brief touches but also sex). For example, people are less willing to put on a sweater when told that a complete stranger has worn it (Rozin et al., 2000). Further, the more aversive the associations that are connected with this stranger, the more disgusting it will become. For example, more people refused to put on the sweater when it had belonged to a man with only one leg or to a murderer (Rozin, Markwith \& McCauley, 1994). 
The fourth category of disgust is socio-moral disgust. This category is the least well defined, but seems to contribute to the conservation of social values. Stimuli in this group are moral violations that are not necessarily disgusting themselves, but are so because the violation breaks certain group norms that apply to a (sub) culture (e.g., pedophilia or racism). Most of the violations in this category have a great deal of overlap with the other disgust categories. Of these four domains, core and animal-reminder disgust are the most widely established forms of disgust. Therefore, this dissertation will focus on these types of disgust.

\section{Differential relations between disgust and psychopathology}

Accumulating evidence suggests that core and A-R disgust may be differentially associated with psychopathology. For example, spider phobia has been associated with heightened levels of a propensity for core disgust, whereas blood phobia has been related most strongly with a propensity for A-R disgust (de Jong \& Merckelbach, 1998; Olatunji et al., 2006). Sawchuk et al. (2000) corroborated these findings by showing that blood-injection phobic individuals displayed a stronger disgust propensity for A-R stimuli than spider phobic individuals or non-phobic individuals, whereas no differences were noted between these groups for core disgust stimuli. It has recently been emphasized that since disgust represents a multi-dimensional construct, proper assessment would require the inclusion of self-report, physiological, behavioral, and cognitive indices (Olatunji \& Sawchuk, 2005). Germane to this, one could speculate that core and animal-reminder disgust are characterized by a differential physiological pattern.

This speculation may be particularly relevant, as it may provide an explanation for disorderspecific symptomatology. For example, a unique characteristic of blood phobia is vasovagal fainting in the presence of phobic stimuli, symptoms that tend to be absent in other specific phobias. Vasovagal fainting is generally associated with the diphasic response, which is defined as an initial rise in blood pressure, followed by an abrupt drop. The initial rise would be the result of sympathetic activity, whereas the sudden drop is associated with sympathetic withdrawal and / or parasympathetic activation of the cardiovascular branch of the ANS, which in turn may give rise to feelings of dizziness. As experiencing disgust is associated with activity in the parasympathetic branch of the autonomic nervous system (Levenson, 1992), it may be that propensity for (A-R) disgust is associated with fainting in blood phobia (Page, 1994; 2003). In line with this view, it was found that diphasic response patterns in response to A-R disgust stimuli were most pronounced in blood fearful persons with relatively high levels of disgust propensity (Page, 2003). However, it should be acknowledged that findings regarding the relation between disgust propensity and the diphasic response in blood phobia are far from consistent in the current literature (e.g, Connolly, O'Neill, Flessner, \& Olatunji, 2006; Gerlach et al., 2006; Olatunji, Williams, Sawchuk, \& Lohr, 2006; Ritz et al., 2005).

Core disgust is presumed to originate from a withdrawal response that occurs during the prospect of oral incorporation of potential contaminants (Rozin et al., 1999). As the digestive branch of the autonomic nervous system is associated with expelling and preventing contaminants from entering the body, core disgust may activate this digestive branch rather 


\section{CHAPTER 1}

than the cardiovascular branch of the ANS (e.g., increases in saliva production, tendency to vomit). A first study confirmed that exposure to core disgust stimuli (both a film clip and mental imagery) evoked an increase in saliva production (de Jong, Peters \& Vanderhallen, 2002). This is not only indicative of parasympathetic nervous system activity, but also suggests that core disgust related activity in the digestive system may indeed be involved in spider phobia (Boon, 2002). Unfortunately, only few studies have been conducted on the physiological reactivity during disgust and on the physiological components of these two disgust types in particular.

\section{Disgust sensitivity}

In addition to disgust propensity, another component that may be of particular importance in the etiology of certain forms of psychopathology is disgust sensitivity. In the context of psychopathology, it may not only be important how readily people respond with the emotion of disgust (disgust propensity), but also how unpleasant people consider the experience of disgust (disgust sensitivity).

It has already been shown in anxiety research that anxiety sensitivity interacts with anxiety expectancies to predict fear behavior (Reiss, 1991; Taylor, 1993). Disgust propensity and sensitivity may interact in a similar way to predict psychopathological complaints. Therefore, it is important to examine both constructs in relation to psychopathology. Disgust propensity is usually assessed via questionnaires, of which the Disgust Questionnaire (DQ; Rozin, Fallon, \& Mandell, 1984), Disgust Emotion Scale (DES; Walls \& Kleinknecht, 1996), and the Disgust Scale (DS; Haidt et al, 1994) are the most widely used in disgust research. Yet, psychometric evaluations of these indices are scarce. Meanwhile, it is known in the literature that the DS, for example, is an internally consistent measure for global disgust propensity whereas internal consistency is low for its subscales which are proposed to represent various disgust domains (Haidt et al., 1994). Since these disgust domains may be differentially associated with various disorders (de Jong \& Merckelbach, 1998), it is important to assess whether these indices can indeed be used to examine separate disgust domains (e.g., core and A-R disgust). Recently, Olatunji et al. (2007) revised the DS. To examine whether the DS-R indeed displays superior psychometric qualities, further validation studies are essential.

Further, although these indices appear valid and reliable indices for measuring global disgust propensity, an index to measure disgust sensitivity is not yet available. Lastly, as the current indices all examine disgust in relation to specific disgust elicitors, inflated correlations may occur between these questionnaires and indices of psychopathology due to items on similar objects in both questionnaires.

Therefore, an instrument that measures both disgust propensity and disgust sensitivity irrespective of disgust elicitors may provide a valuable addition to the present arsenal of available instruments. Hereto, the Disgust Propensity and Sensitivity Scale (DPSS) has recently been devised (Cavanagh \& Davey, 2000). Although preliminary findings indicate that the DPSS indeed measures two separate factors (Cavanagh \& Davey, 2000), further validation is needed in order to be able to draw more definite conclusions. 


\section{Disgust sensitivity and psychopathology}

Both factors disgust propensity and disgust sensitivity may be differentially related to various conditions. High levels of disgust propensity may enlarge the probability of particular stimuli to acquire a disgust-evoking status. Certain stimuli may more readily acquire a disgust-evoking status in people with a high propensity compared to people with low disgust propensity. Hence, this could explain the often reported relationship between disgust propensity and fear of specific objects (e.g., spiders) since, in line with the Disease Avoidance Model, these heightened levels of experienced disgust for these stimuli could signal the individual that these stimuli are potentially hazardous to our health. In addition to this, elevated levels of disgust sensitivity may motivate individuals to avoid specific situations at the prospect of experiencing the unpleasantly evaluated emotion of disgust.

As discussed above, disgust propensity has been found to be important in the etiology of a wide range of psychopathological complaints. Given the potential theoretical importance of disgust sensitivity, a logical next step would be to examine the relative contribution of both factors to psychopathology. It could be that disgust propensity and disgust sensitivity are differentially related to psychopathology. Disgust sensitivity could be especially involved in psychopathologies that are related to intrinsically and universally disgusting stimuli (e.g., vomit, faeces). Although these stimuli are universally disgusting, most people are not characterized by a phobia. It may be that disgust sensitivity could be crucial for an active avoidance of these objects and all situations associated with them. In line with this idea, recent reports indeed suggest that individuals with emetophobia (i.e., fear of vomiting) avoid being disgusted in an attempt to avoid related feelings of nausea (Lipsitz et al., 2001; Veale \& Lambrou, 2006). Thus, emetophobia may present a disorder where disgust sensitivity is more important to psychopathological complaints than disgust propensity.

Disgust propensity could be relatively more important in disorders pertaining to stimuli that are not intrinsically disgusting (e.g., spiders, snakes, sex). Here, heightened levels of disgust propensity may increase the likelihood that, for example, a spider will represent a potential contaminant to the individual such that the unwanted approach of a spider evokes fear more rapidly.

Following on from this, disgust sensitivity would be more critically involved in emetophobia or blood phobia, while disgust propensity would be relatively more important in small animal phobias, obsessive compulsive disorders with cleaning compulsions and sexual dysfunctions. Although the concept of disgust propensity has been studied in relation to various disorders, the concept of disgust sensitivity in relation to psychopathological concerns has rarely been explored at all. Currently, to our knowledge, only one study has been conducted and found disgust sensitivity correlated with health anxiety (Davey \& Bond, 2005). Further investigation on differential associations of disgust propensity and disgust sensitivity with psychopathological symptoms is required, since this could lead to a more accurate conceptualization of these mental disorders. 


\section{CHAPTER 1}

\section{UCS-expectancy bias}

Lastly, cognitive models have placed emphasis on the role of cognitive biases in the genesis and maintenance of psychopathological complaints (Davey, 1997). Accordingly, recent findings showed that in high disgust prone individuals, an attentional bias could be found toward disgust-related words on both the explicit level (Charash \& McKay, 2002) and the implicit level (Charash, McKay, \& DiPaolo, 2006). Another cognitive bias is the UCS-expectancy bias, which can be defined as the tendency to over-associate fear-relevant conditioned stimuli (CS) with the occurrence of aversive unconditioned outcomes (UCS) (e.g., Davey, 1992; Tomarken, Mineka \& Cook, 1989). Certain stimuli may become representations of unwanted, aversive and catastrophic consequences. Previous studies already demonstrated that UCS-expectancy biases are involved in snake phobia (McNally \& Heatherton, 1993), flight phobia (Pauli, Wiedemann, Montoya, 1998), and panic disorder (Wiedemann, Pauli \& Dengler, 2001). UCS-expectancy biases may maintain phobic complaints by initiating a pattern of behavioral avoidance for specific fear-relevant stimuli (e.g., Whittal \& Goetsch, 1997; Jones \& Menzies, 2000), which will logically reduce the opportunity for the extinction of fear.

Most studies on UCS-expectancy biases have only used harm-related outcomes to represent the unwanted aversive outcome (UCS). If disgust propensity and disgust sensitivity are involved in the etiology of disorders (e.g., spider phobia and blood phobia) representations of a disgusting outcome may be more relevant. A first exploration on the relevance of studying disgust-related outcomes indicated that certain animal types were selectively associated with specific outcomes, more specifically, fear-relevant high predatory animals (e.g., sharks) were selectively associated with a harm-related consequence (shock), fear-relevant but low predatory animals (e.g., maggots) with a disgust consequence (nauseating drink), and neutral animals (e.g., rabbits) with neither aversive consequence (Davey et al., 2003). The inclusion of a disgust-related outcome appeared relevant and may more accurately reflect the type of expectancies that individuals hold for various types of stimuli.

Cognitive biases are assumed to contribute to causation and maintenance of phobic complaints (Davey, 1997). Given the importance of contamination-based preoccupations in several forms of psychopathology, it is reasonable to assume that disgust-related expectancy biases rather than fear-related expectancy biases also contribute to specific types of psychopathology. In accordance with this, recent laboratory work showed that disgust-related outcome expectancies following OCD-relevant stimuli were enhanced in participants with high levels of obsessive compulsive complaints (Olatunji, Lohr, Williams, \& Sawchuk (2006).

Previous research already indicated that cognitive biases could be successfully influenced via treatment (de Jong et al., 1992). It has also been shown that residual cognitive biases after successful treatment have predictive validity for the return of complaints at two-years follow-up (de Jong et al., 1995). It remains to be determined whether different types of residual UCSexpectancy biases exert a separate influence on the risk of relapse. If disgust-related expectancy biases rather than fear-related expectancy biases are crucially involved in certain pathologies, then it would be vital to investigate the relative contribution of residual UCS- 
expectancy biases after successful treatment for both harm- and disgust-related outcomes in the return of psychopathological complaints.

\section{Dissertation outline}

In the discussion above, several important issues have been postulated that remain to be investigated. Hence, the current dissertation will address these present gaps in disgust research. First, a series of validation studies will be conducted on the recently revised version of the Disgust Scale (DS-R), as well as on a relatively new scale in disgust research, the Disgust Propensity and Sensitivity Scale (DPSS). In Chapter 2, the recently proposed three-factor model of the DS-R will be examined using confirmatory factor analyses. In Chapter 3, the DPSS will be examined with regard to its factor structure. Next, it will be explored whether individual variation in disgust propensity and disgust sensitivity can predict physiological reactivity during a disgust-evoking film clip as well as behavioral withdrawal for disgusting stimuli in a series of behavioral experiments (Chapter 4). In chapter 5, it will be explored whether individual differences in disgust propensity and disgust sensitivity can predict reactivity on an index for behavioral withdrawal, the eye blink startle response. Following this, it will be investigated whether the construct of disgust sensitivity has additional value next to the already known factor of disgust propensity in relation to specific types of psychopathology, such as blood phobia, spider phobia, eating disorders, obsessive compulsive disorder (Chapter 6), and emetophobia (Chapter 7). Additionally, the current dissertation will focus on whether core- and animal-disgust can be distinguished by separate physiological response patterns, such as the previously hypothesized distinct patterns of activity in the digestive and cardiac branches of the autonomic nervous systems and whether the cardiovascular activity is relatively more pronounced in blood phobic participants (Chapter 8).

In line with the idea that cognitive biases may be important to psychopathology, it will be explored whether participants with elevated levels of spider and blood phobia are indeed characterized by disgust-related expectancy biases. In Chapter 9, it will be investigated whether spider fearful individuals display a disgust-related UCS-expectancy bias, while in Chapter 10, it will be examined whether blood fearful individuals demonstrate a disgust-related UCSexpectancy bias. Moreover, it will be examined whether UCS-expectancy biases for both harmand disgust-related outcomes can effectively be reduced via treatment. Additionally, the relative contribution of harm- and disgust-related expectancy biases to a relapse of phobic complaints later in life will be investigated (Chapter 11).

In line with the view that disgust presents a multidimensional construct, most of the studies in this dissertation will adopt a multi-modal approach (Olatunji \& Sawchuk, 2005; Olatunji, Lohr, Sawchuk, \& Tolin, 2006), which implicates that various parameters should be measured for a reliable assessment (i.e., self-report, physiology, behavioral tests and cognitions) as these together form the multidimensional construct of disgust. For example, upon confrontation with the outer sleeve of this dissertation, you may have cried out in disgust (i.e., self-report), felt your stomach turn and felt faint (i.e., physiology), flung it in the corner of your room 


\section{CHAPTER 1}

(i.e., behavior), and expected gruesome studies to be presented inside (i.e., cognitions). Accordingly, similar assessments will be used throughout this dissertation.

Based on the sleeve and this introductory text, you will by now most certainly have developed specific expectations of your own for the upcoming chapters. Our own expectations were: a) the DS-R and the DPSS are both valid and reliable instruments, b) disgust propensity and sensitivity differentially contribute to the before mentioned psychopathological complaints, c) core and animal-reminder disgust will prove distinct categories of disgust with separate physiological reactivity, d) disgust-related UCS outcome expectancies are involved in spider phobia and blood phobia, e) these UCS-expectancy biases can be successfully influenced via treatment, $\mathrm{f}$ ) there is a reciprocal relationship between UCS-expectancy biases and certain types of psychopathology. In the final section of this dissertation (Chapter 12), the major findings regarding these hypotheses will be integrated and discussed. 


\section{Chapter 2}

The Disgust Scale-R: A valid and reliable index to investigate separate disgust domains? 


\section{CHAPTER 2}

\section{Abstract}

The Disgust Scale (DS; Haidt et al., 1994) is the most widely used instrument for assessing disgust propensity (i.e., individual tendency to experience disgust). Yet, psychometric evaluations of the DS are scarce while the literature shows that the reliability of its subscales are unacceptably low. Therefore, a recent study revised the DS and suggested to reduce the number of subscales from eight to three (DS-R; Olatunji et al., 2007-a). This study is a first exploration of this three-factor solution in an independent sample. A large group of students from Maastricht University $(n=535)$ and the University of Groningen $(n=432)$ completed the DS-R and two other disgust propensity indices, the Disgust Questionnaire (Rozin, Fallon, \& Mandell, 1984) and the Disgust Emotion Scale (Walls \& Kleinknecht, 1996). The results indicated that none of the models demonstrated good fit, and all multiple factor solutions suffered low internal consistencies. Only a one-factor model proved internally consistent. Several suggestions were made for further refinement of the DS-R. 
Disgust propensity (i.e., the individual tendency to respond with the emotion of disgust) has been frequently studied in relation to psychopathology. Accumulating evidence suggests that disgust propensity may be involved in the etiology of a wide range of disgust-relevant psychopathology, like specific fears (de Jong \& Merckelbach, 1998; Page, 1994), obsessive compulsive disorder (Olatunji, Williams, Lohr, \& Sawchuk, 2005), eating disorders (Troop, Murphy, Bramon, \& Treasure, 2000; Troop, Treasure, \& Serpell, 2002), and sexual dysfunctions (de Jong, van Overveld, Weijmar-Schulz, Peters, \& Buwalda, in press).

Although several disgust types have been identified in the literature, only two are wellexamined, in specific, core disgust and animal-reminder disgust (Rozin, Haidt, \& McCauley, 2000). Core disgust involves disgust that is experienced at the prospect of oral incorporation of disgusting stimuli (e.g., rotting foods), whereas animal-reminder disgust (A-R) involves an aversion for stimuli that remind humans of their animal nature. Previous studies showed that these two disgust types can be empirically established (de Jong \& Merckelbach, 1998; Olatunji, Sawchuk, de Jong, \& Lohr, 2005) and that a disgust propensity for core and A-R disgust may be related differentially to psychopathology (de Jong \& Merckelbach, 1998; Koch, O’Neill, Sawchuk, \& Connolly, 2002).

However, current indices generally do not measure these latent concepts (core and A-R disgust) but rather measure disgust propensity in a more context-bound manner. The Disgust Scale (Haidt, McCauley, \& Rozin, 1994) is the most widely used instrument on disgust propensity. Yet, despite its widespread use, psychometric evaluations of the Disgust Scale are scarce. This may be particularly troublesome, as studies consistently report that although internal consistency for the total index is good ( $a=.87$; Druschel, \& Sherman, 1999; .84; Haidt et al., 1994; .74; Olatunji, Smits, Connolly, Willems, \& Lohr, 2007-b), it is consistently within the low to moderate range for its subscales (a's = .34 - .64; Haidt et al., 1994; .40-.68; Olatunji, Williams, Lohr, \& Sawchuk, 2005). Further, although the DS measures disgust for various elicitors, it does not examine core or A-R disgust. Consequently, it is important to examine whether the DS and its subscales could not better be reduced to less subscales, covering larger domains of disgust (core/A-R) rather than eight distinct and less reliable domains.

A first exploration of this provided preliminary support. A recent study investigated correlations between the DS and blood-injury symptoms (Olatunji, Sawchuk, de Jong, \& Lohr, 2006). Most important for the present context, six of the eight DS-subscales were assigned into two subscales measuring core (i.e., Food, Animals, Body Products) and A-R disgust (i.e., Death, Envelope Violations, Sex). Structural equation modeling showed that domain-specific correlations were observed between these two subscales and blood-injury symptoms, so dividing the DS into two large factors appeared valid. Moreover, the model containing these two factors fitted the data well, with the exception of the DS-Sex subscale (Olatunji et al., 2006). Consequently, in a series of studies, Olatunji et al. (2007-a) recently revised the DS and suggested several adjustments. First, seven items were removed from the Disgust Scale due to redundancy or troublesome psychometric aspects (e.g., the Sex subscale). Second, using confirmatory factor analyses, a two-factor solution (Core, A-R) fitted the data significantly better than a one-factor model but a three factor solution (Core, A-R, Contamination) proved 


\section{CHAPTER 2}

even superior. As contamination potency is an important characteristic of disgust stimuli, particularly in the context of psychopathology (e.g., de Jong \& Muris, 2002), this three-factor model could be potentially important.

Thus, the aim of the present study was to determine whether assigning the items of the DS into three subscales investigating Core, A-R, and Contamination fits the data significantly better than the original subscale distribution and a two-factor solution (i.e., Core and A-R) in an independent sample. To investigate concurrent validity of the DS-R and its subscales, the Disgust Emotion Scale (DES; Walls \& Kleinknecht, 1996) and the Disgust Questionnaire (DQ; Rozin, Fallon, \& Mandell, 1984) were also administered.

\section{Methods}

\section{Participants}

Students from the faculties of Psychology, Health Sciences, and Medicine at Maastricht University $(n=535 ; 443$ women, 92 men) and the University of Groningen $(n=432 ; 316$ women, 116 men) were recruited. Their mean age was 20.4 years $(S D=2.4$; range: $17-39$ years).

\section{Materials}

Disgust Scale (DS; Haidt et al., 1994). The DS consists of 32 items that assess an individual's disgust propensity across eight domains: Animals, Bodily Products, Body-Envelope Violations, Death, Food, Hygiene, Sex, and Sympathetic Magic. On the first sixteen items, participants indicate using a dichotomous scoring format $(0=$ no, $1=$ yes $)$ whether a series of statements applies to them. On the second part, participants rate a three-points Likert-type scale $(0=$ 'not disgusting at all', .5 = 'slightly disgusting', $1=$ 'very disgusting') how disgusted they would be when confronted with a series of stimuli. Next, a total score (range: 0-32) and eight subscales (range: $0-4)$ can be calculated. Alpha is high for the total score (.84; Haidt et al., 1994), but is poor for the subscales (.34 - .64; Haidt et al., 1994; .40 - .68; Olatunji, Williams, Lohr, \& Sawchuk, 2005). Recent work suggested to shorten the DS to 25 items with only three subscales. The three subscales and a total score of this 25-item DS-R (Core, A-R, Contamination) can be derived from this 32-item version. The currently used version was translated into Dutch by the authors and cross-translated into English by a professional translator.

Disgust Emotion Scale (DES; Walls \& Kleinknecht, 1996); This 30-item questionnaire measures disgust propensity for five types of stimuli: Animals (DES-An), Rotting Foods (DESRot), Smells (DES-Sm), Mutilation and Death (DES-Mut), and Injections and Blood Draws (DESInj). The first three may be qualified as core disgust, while the latter two appear conceptually associated with Animal-Reminder disgust. On a scale from 0 ( $=$ 'no disgust or repugnance at all') to 4 ('extreme disgust or repugnance'), participants rate how much disgust they would 
experience when confronted with a specific object. It is an internally consistent questionnaire (all a's > .73; Sawchuk, Lohr, Westendorf, Meunier, \& Tolin, 2002).

Disgust and Contamination Sensitivity Questionnaire (DQ; Rozin, Fallon, \& Mandell, 1984); The DQ assesses disgust propensity for core disgust. The participants rate the tendency to reject 24 desirable and perfectly edible food items upon contamination with disgusting objects. On a scale from 1-9 (1 = 'not at all', 9 = 'very much'), participants indicate to what extent they are still willing to eat the items after contamination. Next, a total score can be obtained (range 24-216; high scores reflect low disgust propensity). It is internally consistent ( $a=.72-.86$, Davey, 1994).

\section{Procedure}

For three consecutive years, a large sample of students from Maastricht University was invited to participate in a questionnaire study. During the third administration, a similar study was performed at the University of Groningen. In Maastricht, administration of the Dutch version of the DS occurred in groups (max. 25 participants per group) in one of the conference rooms at our department. In Groningen, participants completed the DS in a lecture theatre setting. All participants were recruited via advertisements in the university papers and posters throughout the university buildings, and completed a series of questionnaires*. The DS and DQ were completed by all participants, while the DES was only administered in two of the Maastricht studies $(n=487)$. Participants were informed that they could win 50 euros through a lottery. The study was performed as part of another project (i.e., van Overveld et al., 2006).

\section{Data reduction and analysis}

The data from four data sets were pooled into one large sample. Following recommendations of Schafer and Graham (2002), regression analyses were used to compute the missing values using the Statistical Package for Social Sciences (SPSS; version 12.0.1. Next, the DS-R subscales were calculated from the DS scores. For the one-factor model, all 25 DS-R items were summed into a total score. The two- and three-factor models were calculated as proposed by Olatunji et al. (2007-a) with one exception: as all models should be nested, item 16 ("It would bother me to sleep in a hotel room if I knew that a man had died of a heart attack in that room the night before") was included in the A-R subscale instead of in the Core subscale. Thus, the A-R scale was identical for the two- and three-factor solution. For the seven-factor model, items were included in the original subscales by Haidt et al. (1994). Thus, compared with the original 32-item distribution, the Sex-subscale was not calculated and DS-R subscales Food, Body and Envelope Violations all contained one item less. First, the descriptives of all questionnaires were calculated for the entire research population $(N=967)$. Second, a confirmatory factor analyses (CFA) was performed to determine whether the proposed three factor model would fit the data

\footnotetext{
* On behalf of another study presented elsewhere (van Overveld, de Jong, Peters, Cavanagh, \& Davey, 2006), Dutch versions of the following questionnaires were also administered: the Disgust Propensity and Sensitivity Scale-Revised, Blood Injury Phobia Questionnaire, and the Fear of Spiders Questionnaire.
} 


\section{CHAPTER 2}

significantly better than a one- and a two-factor solution, and whether the various DS-R models demonstrate good model fit. Hereto, polychoric correlation matrices and asymptotic covariance matrices were obtained by PRELIS. Using LISREL 8.54 (Jöreskog \& Sörbom, 2002), the various factor models were examined with confirmatory factor analysis. The Weighted Least-Squares estimation was used due to the discrete scoring format of the DS-R (see also Olatunji et al., 2007-a). Further, latent variables were free to correlate and secondary loadings of items were not permitted. Additionally, item residuals were allowed to correlate only if items belonged to identical subscales. Third, bivariate correlations between all trait disgust indices and their subscales were calculated to establish whether the DS-R and its (suggested) subscales correlate meaningfully with the other stimulus-bound disgust propensity indices. Thus, DS-R Core was expected to display stronger associations with DQ-total, DES-Food, and DES-Animals, while the DS-R A-R was expected to demonstrate stronger associations with DES-Mut and DES-Inj.

Table 1.

Summary of the means, standard deviations and reliability coefficients for all trait disgust indices.

\begin{tabular}{|c|c|c|c|c|}
\hline & Mean & SD & Range & Cronbach's alpha \\
\hline DS-R total & 17.57 & 4.77 & $3-32$ & .70 \\
\hline - DS-R-Ani & 3.72 & 1.26 & $0-6$ & .30 \\
\hline - DS-R-Food & 2.24 & .13 & $0-5$ & .14 \\
\hline - DS-R-Body & 2.15 & 1.03 & $0-4$ & .31 \\
\hline - DS-R-Hyg & 2.36 & 1.08 & $0-5$ & .21 \\
\hline - DS-R-Env & 2.75 & .91 & $0-4$ & .12 \\
\hline - DS-R-Death & 2.59 & 1.53 & $0-6$ & .43 \\
\hline - DS-R-Magic & 1.76 & 1.10 & $0-6$ & .28 \\
\hline \multicolumn{5}{|c|}{ DS-R two factor solution } \\
\hline - DS-R-Core & 11.56 & 3.40 & $1-22$ & .61 \\
\hline - DS-R-AR & 6.01 & 2.18 & $0-11$ & .52 \\
\hline \multicolumn{5}{|c|}{ DS-R three factor solution } \\
\hline - DS-R-Core & 10.12 & 2.90 & $0-18$ & .59 \\
\hline - DS-R-AR & 6.01 & 2.18 & $0-11$ & .52 \\
\hline - DS-R-Cont & 1.44 & 1.17 & $0-7$ & .27 \\
\hline DES-total & 35.01 & 15.10 & $5-86$ & .91 \\
\hline - DES-Ani & 3.84 & 3.19 & $0-15$ & .64 \\
\hline - DES-Rot & 8.05 & 4.49 & $0-21$ & .91 \\
\hline - DES-Sm & 9.41 & 4.14 & $0-23$ & .84 \\
\hline - DES-Mut & 9.59 & 4.75 & $0-23$ & .84 \\
\hline - DES-Inj & 4.13 & 4.74 & $0-24$ & .87 \\
\hline $\mathrm{DQ}$ & 134.76 & 36.08 & $33-214$ & .94 \\
\hline
\end{tabular}

SD = Standard Deviation, DES = Disgust Emotion Scale, DQ = Disgust Questionnaire, DS-R = Disgust Scale-Revised, Ani $=$ Animals, Body $=$ Body Products, Hyg $=$ Hygien, Env $=$ Body-Envelope Violations, Magic $=$ Sympathetic magic, Cont $=$ Contamination, Rot $=$ Rotting Food, $\mathrm{Sm}=$ Smells, Mut $=$ Mutilation and Death, Inj $=$ Injection and Blood Draws. 


\section{Results}

\section{Descriptives}

Table 1 summarizes the means, standard deviations, and the actual range for all questionnaires in the present sample (original DS-R, the two-factor distribution of the DS-R, the proposed three-factor solution, DES, and DQ). In accordance with the literature (Haidt et al., 1994), internal consistency was acceptable for the total score of the DS-R (alpha $=.70$ ), while low for the original subscales. For the two and three factor models, the internal consistency of the respective subscales was also low. Additionally, a series of independent $t$-tests revealed that women generally endorsed significantly higher scores with regard to the total scores of the DES $(t(485)=-3.20 ; p<.001)$, DQ $(t(963)=6.61 ; p<.001)$, and the DS-R (and all its subscales; all $p$ s $<.001)$ with the exception of the Contamination subscale $(t(964)=-1.00 ; p=.32)$.

\section{Confirmatory factor analysis}

Model fit of the various models was examined using absolute and incremental fit indices (see also Kline, 1998). The chi-square ratio ( $\left.x^{2} / d f\right)$ is an absolute fit index. In general, a ratio below 3 indicates that a model has good fit. In the present study, the ratios for all possible models indicate that they do not fit the data well. For the incremental fit indices, the cut-off criteria for good fit as recommended by Hu and Bentler (1999) were used: 1. Comparative Fit Index (CFI) $>.95,2$. Tucker-Lewis Index (TLI) > .95, and 3. Root Mean Square Error of Approximation (RMSEA) <.06. As can be seen in Table 2, model fit was poor for all models.

The chi-squares difference test is the standard test for a comparison of nested models. It was used here to assess whether compared to the original or previous model, improvements to the current model were significant. Compared to the one-factor solution, the two-factor model fitted the data significantly better $\left(X_{\text {diff }}^{2}(1)=7.80 ; p<.01\right)$. The three-factor model fitted the data significantly better than the two-factor model $\left(x^{2}\right.$ diff $\left.(2)=45.66 ; p<.01\right)$. Lastly, the original distribution (seven-factor model) fitted the data significantly better than the threefactor model $\left(X_{\text {diff }}^{2}(18)=79.40 ; p<.01\right)$. Additionally, the Expected Cross-Validation Index (ECVI) indicates a descriptive evaluation of competing models where the model with the lowest value generally fits best. Here, the seven-factor model displayed the lowest ECVI value. So, in line with earlier research (Olatunji et al., 2007-a), the three-factor model was superior to a one and two-factor solution, although it must be noted that none fitted the data significantly better than the original distribution and all models actually showed poor fit. 
CHAPTER 2

Table 2.

Goodness of Fit indices for all DS-R models.

\begin{tabular}{llllllll}
\hline & $\mathrm{X}^{2}$ & d.f. & $\mathrm{X}^{2} /$ d.f. & CFI & TLI & RMSEA & ECVI \\
\hline One factor & 1796.14 & 275 & 6.53 & .66 & .63 & .07 & 1.96 \\
Two factors & 1788.34 & 274 & 6.53 & .66 & .63 & .08 & 1.96 \\
Three factors & 1742.68 & 272 & 6.41 & .67 & .64 & .07 & 1.92 \\
Seven factors & 1663.28 & 254 & 6.55 & .68 & .62 & .08 & 1.87 \\
\hline Valid $N$ & & & & & & & 966
\end{tabular}

Note: CFI = Comparative Fit Index, TLI = Tucker-Lewis Index, RMSEA = Root Mean

Square Error of Approximation, ECVI = Expected Cross-Validation Index

\section{Convergent validity}

To assess convergent validity of the DS-R and its subscales, a series of bivariate correlations were computed between the DS-R and the other disgust propensity indices (DES, DQ). Table 3 shows that the DS-R correlated moderately with the total scores on the DES $(r=.47)$ and the DQ $(r=-.41)$. A similar correlation was observed between the DQ and DES $(r=-.38)$. More interestingly, for the two and the three-factor distributions, the Core subscales of the DS-R correlated best with the DQ total score (measuring core disgust) and with various DESsubscales that may be associated with core disgust (e.g., Smells, Rotting Foods, Animals) while the A-R subscale correlated best with the Mutilation subscale of the DES and with the Injections-subscale.

Table 3.

Bivariate correlations between the original and revised DS with the other trait disgust indices (DES, DQ).

\begin{tabular}{|c|c|c|c|c|c|c|c|}
\hline & $\begin{array}{l}\text { DES } \\
\text { total }\end{array}$ & Ani & Rot & $\mathrm{Sm}$ & Mut & Inj & $\begin{array}{l}\mathrm{DQ} \\
\text { total }\end{array}$ \\
\hline DS-R total & $.47 * *$ & $.35^{* *}$ & $.32 * *$ & $.37 * *$ & $.41 * *$ & $.24 * *$ & $-.41 * *$ \\
\hline DS-R Ani & $.38 * *$ & $.29 * *$ & $.31 * *$ & $.30 * *$ & $.32 * *$ & $.14 * *$ & $-.30 * *$ \\
\hline DS-R Food & $.33 * *$ & $.23 * *$ & $.34 * *$ & $.30 * *$ & $.19 * *$ & $.12^{* *}$ & $-.23 * *$ \\
\hline DS-R Body & $.13^{* *}$ & .05 & .09 & $.27 * *$ & .05 & $.03 * *$ & $-.21 * *$ \\
\hline DS-R Hyg & .07 & $.13 * *$ & .03 & $.10 *$ & .01 & .01 & $-.19 * *$ \\
\hline DS-R Env & $.16^{* *}$ & .04 & -.01 & .03 & $.31 * *$ & $.16^{* *}$ & $-.10 * *$ \\
\hline DS-R Death & $.33 * *$ & $.28^{* *}$ & $.14 * *$ & $.15^{* *}$ & $.35^{* *}$ & $.25 * *$ & $-.25 * *$ \\
\hline DS-R Magic & $.20 * *$ & $.13 * *$ & $.16^{* *}$ & $.14 * *$ & $.18^{* *}$ & $.10 * *$ & $-.37 * *$ \\
\hline \multicolumn{8}{|c|}{ DS-Rtwo factors } \\
\hline DS-Core & $.43 * *$ & $.32 * *$ & $.35 * *$ & $.42 * *$ & $.29 * *$ & $.15^{* *}$ & $-.42 * *$ \\
\hline DS-A-R & $.32 * *$ & $.22 * *$ & $.11^{*}$ & $.12 * *$ & $.40 * *$ & $.26 * *$ & $-.24 * *$ \\
\hline \multicolumn{8}{|c|}{ DS-R three factors } \\
\hline DS-R Core & $.41^{* *}$ & $.28^{* *}$ & $.37 * *$ & $.44 * *$ & $.26 * *$ & $.13^{* *}$ & $-.38 * *$ \\
\hline DS-R AR & $.32 * *$ & $.22 * *$ & $.11^{*}$ & $.12^{* *}$ & $.40 * *$ & $.26 * *$ & $-.24 * *$ \\
\hline DS-R Cont & $.17^{* *}$ & $.19 * *$ & .06 & .08 & $.17^{* *}$ & $.10 *$ & $-.27 * *$ \\
\hline Valid $N$ & 487 & & & & & & 966 \\
\hline
\end{tabular}

$\mathrm{SD}=$ Standard Deviation, DES = Disgust Emotion Scale, DQ = Disgust Questionnaire, DS = Disgust Scale, Ani = Animals, Body = Body Products, Hyg = Hygien, Env = Body-Envelope Violations, Magic = Sympathetic magic, Cont = Contamination, Rot $=$ Rotting Food, Sm = Smells, Mut = Mutilation and Death, Inj = Injection and Blood Draws.

$*$ = significant at $\mathrm{p}<.05 ; * *=$ significant at $\mathrm{p}<.01$ 


\section{Discussion}

The main findings were: i) model fit was poor for all models, ii) only the one-factor model demonstrated acceptable internal consistency, iii) the two-factor solution correlated differentially with related disgust indices which could indicate that a two-factor solution may also be meaningful.

Previous research showed that the psychometric aspects of the DS, the most widely used instrument to measure disgust propensity, were not very satisfactory. In particular, its subscales lack internal consistency (Haidt et al., 1994; Olatunji, Williams, Lohr, \& Sawchuk, 2005). Recently, Olatunji et al. (2007-a) showed that several adjustments critically improved its psychometric properties. First, a number of items was deleted as they were either redundant or detracted performance of the DS. Second, factor analyses revealed that two items displayed too much item overlap with other items, so these items were also removed. Third, using factor analyses, a three-factor solution was constructed, which proved superior to a one- and a twofactor model, but was not compared to the original DS-subscales.

In the current study, the proposed three-factor model fitted the data significantly better than a one-factor model or a two-factor model. This may support earlier work that indicated that a subscale on contamination ideation appears a valid addition reflecting an essential component of disgust stimuli, namely contamination potency (Olatunji, Sawchuk, Williams, \& Lohr, 2005; Olatunji et al., 2007-a). However, internal consistency for the two- and three-factor solutions was in fact unacceptably low. Moreover, model fit of all models was poor. In line with earlier reports (Haidt et al., 1994; Olatunji et al., 2006), only the one-factor model demonstrated acceptable internal consistency. Given the overall poor model fit, it may be recommendable to use only the one-factor solution that measures global disgust propensity since this model can be easily interpreted, requires the least assumptions, and is the only one that appears reliable.

Yet, the present study also replicates earlier work (Olatunji et al., 2005) that the DS may measure core and A-R disgust. In line with that study, DS-R Core was associated most strongly with DES-Smells and DES-Food. The A-R subscale was most strongly associated with DES-Inj and DES-Mut. Contrary to earlier work (Olatunji et al., 2005), however, the A-R scale was also associated with DES-Smells. Given the close association of A-R disgust with death and decay (Rozin \& Fallon, 1987), this may not be surprising. Further, DS-R core was associated most strongly with an index on core disgust (DQ), while DS-R A-R was not.

These indications that the DS-R may indeed measure distinct disgust types (core, A-R) is important since these two disgust types may be differentially involved in psychopathology (for example, core disgust in spider phobia, A-R disgust in blood phobia; de Jong \& Merckelbach, 1998). While for core disgust, an adequate index is already available (DQ; Arrindell, Mulkens, Kok, \& Volkenbroek, 1999), there is currently no such index for A-R disgust. Although the DS-R is assumed to reliably index both core and A-R disgust (Olatunji et al., 2007-a), the present data do not confirm this. However, by implementing several adjustments to the DS-R, a twofactor solution may eventually prove superior to the currently advised one-factor model. 


\section{CHAPTER 2}

Several adjustments might improve the psychometric properties of the DS-R. First, the items of the Contamination-subscale may be removed to ensure a more straightforward interpretation of disgust propensity as it displays low internal consistency and remains difficult too interpret. For example, is an individual's global disgust propensity determined by both disgust type (e.g., core, A-R) and disgust characteristics (contamination)? If so, can a global disgust propensity score still be calculated when an individual indicates a greater disgust for core/A-R stimuli while these stimuli do not necessarily have great contamination potency? It may be useful to restrict the DS-R to measuring either disgust elicitors (core and A-R disgust) or disgust characteristics (contamination potency).

Second, akin to other vulnerability factors, the concept of disgust propensity may exist on a continuum (Olatunji, Sawchuk, de Jong, \& Lohr, 2007-c). If so, then a dichotomous scoring format does not accurately represent reality, which could explain the poor model fit. Therefore, an answering format using a Likert-type scale, which is more in line with a representation of a continuum, would be more appropriate (Olatunji et al., 2007-a). Third, the first half of the DS-R contains a series of 'true / false' statements. Yet, whether the corresponding answer is due to disgust or another emotion remains unclear. It may be recommendable to either include answering categories for both disgust and fear (Olatunji et al., 2007-a), or adapt the DS-R in such a way that one unambiguous question is used for all items (e.g., 'How disgusted would you be in the following situation').

Although the DS-R appears a reliable instrument for measuring global disgust propensity, the present data suggest that it remains uncertain whether the DS-R measures multiple consistent dimensions. Thus, a more suitable index to measure core and A-R disgust may be the DES. A recent validation study in two independent samples in the Netherlands and the United States showed that the DES demonstrated good internal consistency for both the total score and the subscales. Moreover, its factor structure was supported using factor analyses. Further, convergent validity was also good (Olatunji et al., 2007-c).

Several comments are in order with respect to the present study. First, convergent validity but not divergent validity was studied. To attest the validity of the DS-R, it may be important to assess whether its subscales are only associated with related constructs. For example, it may be studied whether DS-R Core disgust is associated with other core disgust scales while it should not be associated with unrelated aspects of other basic emotions such as anger types (for example, the Clinical Anger Scale; Snell, Gum, Shuck, Mosley, \& Hite, 1995).

Second, the present study investigated construct validity but not any other validity types (e.g., predictive validity). To further establish the validity of the DS-R, researchers should investigate whether higher scores on the DS-R subscales (Core, A-R, Contamination) can predict actual behavioral avoidance for that particular type of stimuli using behavioral experiments (e.g., Koch et al., 2002). This may be particularly important given the assumption that elevated levels of a propensity for specific disgust types may be related to clinical avoidance patterns for various stimuli (e.g., spiders; de Jong \& Muris, 2002).

So, at this stage, it appears that a one-factor solution of the DS-R measuring global disgust propensity may be preferable to a two- or three-factor solution. Nevertheless, the possibility arises that two factors can indeed be distinguished using the DS-R since subscales on core and 
A-R disgust correlated differentially with other core and A-R indices. Yet, it should be noted that their internal consistency was low. It is not unlikely that several adjustments to the DS-R may improve its psychometric properties (e.g., a continuous scoring format, and using one question for the entire questionnaire). Such revisions could allow the DS-R to meaningfully distinguish core and A-R disgust. 


\section{Chapter 3}

\section{Disgust propensity and disgust sensitivity: Separate constructs?}




\section{CHAPTER 3}

\section{Abstract $^{+}$}

Studies concentrating on interindividual differences in experiencing disgust have indicated that disgust propensity is associated with certain disorders, such as fear of blood and fear of spiders (de Jong \& Merckelbach, 1998). However, current indices of disgust propensity suffer from conceptual overlap with measures of psychopathology. Moreover, in addition to high levels of disgust propensity, a relatively negative appreciation of experiencing the emotion of disgust (disgust sensitivity) may also be critically involved in psychopathology. To address these issues, the Disgust Propensity and Sensitivity Scale (DPSS) was devised (Cavanagh \& Davey, 2000). This study examined its psychometric qualities. Students $(N=967)$ completed the DPSS, the Disgust Questionnaire (DQ), the Disgust Scale (DS), the Blood-Injury Phobia Questionnaire (BIQ), and the Fear of Spiders Questionnaire (FSQ). The DPSS meaningfully differentiated between disgust propensity and sensitivity. These factors were differentially related to blood and spider fear. The present findings sustain the importance of differentiating between individual differences in disgust propensity and sensitivity as factors that may be independently involved in psychopathology. The DPSS appears a valuable addition to the arsenal of indices presently available in disgust research.

\footnotetext{
+ Published as: van Overveld, W. J. M. , de Jong, P. J., Peters, M. L., Cavanagh, K., \& Davey, G. C. L. (2006). Disgust propensity and disgust sensitivity: separate constructs that are differentially related to specific fears. Personality and Individual Differences, 41, 1241-1252.
} 
There is increasing attention for the potential role of disgust propensity (defined as a general tendency to respond with the emotion of disgust to any given situation) in psychopathology. Using instruments like the Disgust and Contamination Sensitivity Questionnaire (DQ; Rozin, Fallon \& Mandell, 1984), and the Disgust Scale (DS; Haidt, McCauley, \& Rozin, 1994), inflated levels of disgust propensity were found in spider fearful individuals (Tolin, Lohr, Sawchuk, \& Lee, 1997; Sawchuk, Lohr, Tolin, Lee, \& Kleinknecht, 2000), in blood-fearful individuals (de Jong \& Merckelbach, 1998; Sawchuk, Lohr, Westendorf, Meunier, \& Tolin, 2002; Schienle et al., 2003), and in patients with obsessive-compulsive disorder (Muris et al., 2000; Schienle et al., 2003).

In support of their validity, these self-report measures correspond well with actual disgustinduced avoidance behavior in behavioral experiments (Klieger \& Siejak, 1997; Rozin et al., 1999). However, two major drawbacks can be noted. First, they measure disgust for specific elicitors. Hence, inflated correlations may arise between disgust propensity and psychopathology due to items that enquire about similar objects in both questionnaires (Thorpe \& Salkovskis, 1998). For an adequate examination of the relationship between disgust propensity and psychopathology, an instrument that does not suffer from such confounding would be helpful.

Second, the instruments all enquire to what extent people are disgusted by certain stimuli, but not whether they appraise these experiences more negatively. Thus, they measure propensity, but not sensitivity. Yet, for the development of psychopathology, it may not only be relevant how easily people are disgusted (propensity), but also how unpleasant the experience of disgust is to the individual (sensitivity). Anxiety research revealed that anxiety sensitivity interacts with anxiety expectancies to predict fear behavior (Reiss, 1991; Taylor, 1993). In a similar vein, disgust propensity and sensitivity may interact and predict disgust-related psychopathological complaints. Consequently, an instrument measuring both factors would provide a valuable addition to presently available instruments.

Based on these considerations, the Disgust Propensity and Sensitivity Scale (DPSS) was designed in an attempt to cover disgust propensity and sensitivity (Cavanagh \& Davey, 2000), and to measure these characteristics irrespective of particular elicitors (avoiding conceptual overlap with measures of psychopathology). The major goal of this study is to establish its psychometric properties (i.e., factor structure, reliability, convergent validity) and to explore whether propensity and sensitivity may be differentially involved in fear of spiders and fear of blood.

Additionally, fainting is a specific symptom of blood phobia. As disgust appears predominantly involved in blood phobia (Page, 1994, 2003), this may explain why blood fearful individuals faint, while people with other types of specific fears do not. Therefore, it will be examined whether people with a fainting history for blood-injection-injury (BII) stimuli endorse the DPSS differently than non-fainters. 


\section{CHAPTER 3}

\section{Methods}

\section{Participants}

Students at two Dutch universities participated in this study. They were recruited from the faculties of Psychology and Medicine at Maastricht University ( $n=535 ; 443$ women, 92 men) and the University of Groningen ( $n=432 ; 316$ women, 116 men). Mean age was 20.4 years $(S D=2.4$; range: $17-39$ years).

\section{Materials}

Disgust Propensity and Sensitivity Scale (DPSS; Cavanagh \& Davey, 2000). The DPSS measures disgust propensity and sensitivity ${ }^{1}$. Participants are instructed to read 32 posits and mark the answer which is most appropriate to them. The items enquire about the frequency of experiencing certain (bodily) symptoms and their emotional impact, and have to be rated on a scale from 1 (= 'never') to 5 (= 'always'; range: 32-160) (see Table 1 for the complete DPSS). Previous research found the DPSS internally consistent, with alpha coefficients of .89 (Propensity ) and .87 (Sensitivity) (Cavanagh \& Davey, 2000).

Disgust Scale (DS; Haidt et al., 1994). This 32-item questionnaire measures disgust propensity across eight domains: Body-Envelope Violations, Animals, Bodily Products, Hygiene, Sex, Food, Death and Sympathetic Magic. Each domain consists of two true/false type questions, and two items using a scale from 0 (= 'not disgusting at all') to 2 (= 'extremely disgusting'). A total score (range: $0-32$ ) and subscale scores can be calculated. Although alphas for the subscales are poor (.34 to .64; Haidt et al., 1994), alpha is high for the total score (.84; Haidt et al., 1994).

The Disgust and Contamination Sensitivity Questionnaire (DQ; Rozin, Fallon, \& Mandell, 1984); This 24-item questionnaire measures the tendency to reject desirable food items, based on their degree of contamination with disgusting stimuli. Participants rate on a scale from 1 to 9 ( 1 = 'not at all', $9=$ 'very much') whether they would still like to eat a food item after contamination (range 24-216). Its reliability is good (alpha = .72 to .86, Davey, 1994; .80, Mulkens, de Jong, \& Merckelbach, 1996).

Fear of Spiders Questionnaire (FSQ); This questionnaire examines fear of spiders. Participants rate their fear of spiders on 18 items using a scale from 0 (= 'strongly disagree') to 7 (= 'strongly agree'; range: 0-126). The FSQ is an internally consistent index (alpha = .92; Szymanski \& O'Donohue, 1995).

Blood-Injury Phobia Questionnaire (BIQ); This 20-item questionnaire consists of two separate 10-item subscales, BIQ-Fear and BIQ-Fainting. On BIQ-Fear, participants rate on a scale from 0 (= 'no fear') to 4 (= 'maximal fear'; range $=0-40$ ) their fear of 10 BII-stimuli. It appears internally consistent (.82-.87; Merckelbach, Muris, de Jong \& de Jongh, 1999). On BIQFainting, participants rate on a scale from 0 (= 'never') to 2 (= 'often'; range: $0-20$ ) if they have ever fainted in the presence of these BII-stimuli. Internal consistency is satisfactory (.73-.78; Merckelbach et al., 1999). 


\section{DISGUST PROPENSITY AND DISGUST SENSITIVITY}

\section{Procedure}

Three consecutive years, a large sample of students at Maastricht University completed a series of questionnaires. The third time, a parallel study was conducted in Groningen. Administration in Maastricht took place in groups (max. 25 participants) in a conference room. In Groningen, questionnaires were completed in a lecture theatre setting. Participants were recruited via posters and advertisements at the University buildings. They completed Dutch versions of the DPSS, DS, DQ, FSQ, and BIQ. Previous studies indicate that psychometric properties of these translated versions are acceptable (de Jong \& Merckelbach, 1998; Muris et al., 2000). Participants were informed that via a lottery, ten people could win 50 euros. Sixty randomly selected individuals from the last Maastricht study completed the DPSS again after two months. This sample comprised of 9 men, and 51 women. Mean age was 21.6 years $(S D=2.9$; range: 18-39 years)

\section{Results}

\section{Data reduction and analysis}

The data from these studies was pooled into one large sample. Using the Statistical Package for Social Sciences (SPSS), missing values were estimated using regression analyses following recommendations of Schafer and Graham (2002). Only complete questionnaires were included in this analysis $(N=967)$. The dataset was then randomly divided into two separate sets. One set $(n=487)$ was used for exploratory factor analysis, while on the other dataset $(n=480)$ a confirmatory factor analysis was performed. Exploratory factor analysis was used to guide selection of items into two homogenous subscales, equal in length (eight items). Confirmatory factor analysis was used to determine whether these two subscales could be confirmed in an independent sample. For establishing convergent validity, correlations between the DPSS, DS, and DQ were studied in the entire dataset. Regression analyses examined whether scores on DPSS-subscales are predictive of scores on the BIQ-Fear / FSQ. Finally, test-retest reliability was examined.

\section{Construction of subscales}

Cavanagh \& Davey (2000) originally proposed that the DPSS measures two constructsł, therefore, a forced two-factor exploratory factor analysis using principal component analysis with Oblimin rotation was performed. Based on the eigenvalues (which had to be larger than one for a factor to be considered a separate factor) and the scree-plot, these two factors were established, accounting for $35.6 \%$ of total variance. These factors correlated moderately (.54).

₹ The following items were originally assigned to Propensity $(1,2,7,12,13,14,18,20,22,23,26,28,31)$ and Sensitivity $(3,4,5,6,8,9,10,11,15,16,17,19,21,24,25,27,29,30,32)$. 


\section{CHAPTER 3}

Table 1 shows that most items loaded on one of these factors. Next, two homogenous subscales of equal length were constructed. Following the recommendations by Clark and Watson (1995), selection of items occurred on both theory- and data-driven considerations. Subscales were designed to measure constructs propensity and sensitivity broadly, so, not merely items with highest loadings were eligible for selection. Table 1 displays which items were included in the subscales.

\section{Confirmatory factor analysis}

First, polychoric correlation matrices were obtained by PRELIS. Using LISREL 8.54 (Jöreskog and Sörbom, 2002), the two subscales were examined with confirmatory factor analysis using weighted least-squares estimation. Latent variables were free to correlate. Secondary loadings of items were not permitted.

Additionally, item residuals were allowed to correlate only if items belonged to identical subscales. Next, model fit was examined using absolute and incremental fit indices. Both are equally important in evaluating model fit (Kline, 1998). The chi-square test ( $\mathrm{x}^{2} / \mathrm{df}$ ) is an absolute fit index. Generally, a ratio below 3 is considered good. Here, the ratio is 2.7, indicating that the model has a good fit.

For incremental fit indices, cut-off criteria for good fit as recommended by $\mathrm{Hu}$ and Bentler (1999) were used: Comparative Fit Index (CFI) > .95, Tucker-Lewis Index (TLI) > .95, and Root Mean Square Error of Approximation (RMSEA) < .06. According to these criteria, the model consisting of the two subscales has a reasonable fit (see Table 2). Furthermore, the latent factor correlation was high (.78), but did significantly differ from 1 (95\% Confidence Interval: .72-.84).

To test whether the two-subscales structure has additional value compared to a one-factor model, an additional confirmatory factor analysis was conducted (see Table 2). For this onefactor model, all sixteen items were regarded as one large factor. To examine which model fits the data best, a likelihood ratio test was performed, in which the difference in chi-square values of the models was compared. 
DISGUST PROPENSITY AND DISGUST SENSITIVITY

Table 1.

Factor loadings and eigenvalues for the complete DPSS.

\begin{tabular}{|c|c|c|c|}
\hline Item & Description & Propensity & Sensitivity \\
\hline \multicolumn{4}{|c|}{ Included items } \\
\hline 26 & I screw up my face in disgust & .71 & -.22 \\
\hline 20 & Disgusting things make my stomach turn & .68 & .11 \\
\hline 14 & I experience disgust & .66 & -.06 \\
\hline 12 & I feel repulsed & .64 & -.06 \\
\hline 28 & I find something disgusting & .65 & -.09 \\
\hline 31 & When I experience disgust, it is an intense feeling & .52 & .22 \\
\hline 1 & I become disgusted more easily than other people & .49 & -.06 \\
\hline 2 & I avoid disgusting things & .45 & -.05 \\
\hline \multicolumn{4}{|c|}{ Excluded items } \\
\hline 30 & It scares me when I feel faint & -.02 & .68 \\
\hline 19 & It scares me when I feel nauseous & .10 & .68 \\
\hline 11 & When I feel disgusted, I worry that I might pass out & .04 & .62 \\
\hline 15 & I think feeling disgust is bad for me & -.01 & .57 \\
\hline 5 & I think disgusting items could cause me illness / infection. & .01 & .53 \\
\hline 17 & I worry that I might swallow a disgusting thing & .07 & .50 \\
\hline 32 & It embarrasses me when I feel disgusted & .06 & .50 \\
\hline 8 & When I notice that I feel nauseous, I worry about vomiting & .25 & .34 \\
\hline 22 & When I am disgusted, my stomach turns & .65 & .11 \\
\hline 18 & Disgusting things make my skin crawl & .49 & .19 \\
\hline 13 & When I am disgusted, I feel nauseous & .48 & .26 \\
\hline 21 & When I am disgusted, I feel sweaty & .45 & .28 \\
\hline 25 & I find it easy to ignore feelings of disgust & .44 & .14 \\
\hline 27 & I cannot think clearly when I am disgusted & .39 & .26 \\
\hline 24 & Rancid smells distress me. & .37 & .14 \\
\hline 29 & $\begin{array}{l}\text { I worry that I might harm myself trying to avoid something which } \\
\text { disgusts me }\end{array}$ & -.15 & .67 \\
\hline 3 & When I feel disgusted, my bodily sensations frighten me & .02 & .65 \\
\hline 16 & It scares me when I vomit & .01 & .64 \\
\hline 10 & When I am disgusted I feel like I might be contaminated & -.07 & .62 \\
\hline 9 & It scares me when I salivate excessively & .01 & .57 \\
\hline 6 & Disgust can really take me over & .25 & .38 \\
\hline 4 & When I am disgusted, I feel faint & .31 & .35 \\
\hline 7 & I am comfortable handling things others find disgusting & .27 & -.05 \\
\hline 23 & When I feel disgusted, it passes quickly & .21 & .23 \\
\hline \multicolumn{2}{|c|}{ Eigen values } & 9.60 & 1.80 \\
\hline
\end{tabular}

Note: items loaded on a factor if their loading was $>.30$ 


\section{CHAPTER 3}

Table 2.

Goodness of Fit indices for all factor models

\begin{tabular}{llllllll}
\hline & $\mathrm{X}^{2}$ & d.f. & $\mathrm{X}^{2} /$ d.f. & CFI & TLI & RMSEA & ECVI \\
\hline One factor & 345.35 & 103 & 3.35 & .88 & .86 & .070 & .86 \\
Two factors & 278.93 & 102 & 2.74 & .91 & .89 & .060 & .72 \\
\hline
\end{tabular}

Note: CFI = Comparative Fit Index, TLI = Tucker-Lewis Index, RMSEA = Root Mean Square Error of Approximation, ECVI $=$ Expected Cross-Validation Index

The two-subscales model fitted significantly better than the one-factor model $(p<.001)$. Further, the Expected Cross-Validation Index (ECVI) provides a descriptive evaluation of competing models. For the ECVI, the model with the lowest value fits best. Table 2 depicts that the ECVI was lowest for the two-subscales model, indicating that this model fits best.

\section{Reliability analysis}

For the DPSS-subscales, Cronbach's alphas were adequate to good (all > .71; see Table 3). Test-retest reliability was examined using Intra-Class Correlation Coefficients (ICC), and was good for both Propensity (.69) and Sensitivity (.77). For the DQ, Cronbach's alpha was good (.72). Alphas were poor for the DS-subscales (all < .43). Therefore, only DS total scores were used for further analyses (alpha $=.68$ ). For the BIQ and FSQ, alphas were good: .83 (BIQFear), .81 (BIQ-Faint), .82 (BIQ total score) and .97 (FSQ total scores).

Table 3.

Correlation matrix for DPSS and the disgust propensity indices.

\begin{tabular}{|c|c|c|c|c|}
\hline & DPSS Propensity & DPSS Sensitivity & DQ & DS \\
\hline DPSS Propensity & $a=.78$ & & & \\
\hline DPSS Sensitivity & $.59 * *$ & .77 & & \\
\hline DQ & $-.21 * *$ & $-.16 * *$ & .72 & \\
\hline DS & $.37 * *$ & $.29 * *$ & $-.37 * *$ & $.94 * *$ \\
\hline Valid N & & & & 963 \\
\hline
\end{tabular}

** Significant at $p<.01$

On the diagonal axis, Cronbach's alphas are presented

\section{Validity}

Table 3 shows that the DPSS correlated moderately with DQ and DS. As expected, the highest correlations were observed between DPSS-Propensity and the other propensity measures (DS and $\mathrm{DQ})$.

\section{Correlations with psychopathology}

Table 4 shows the DPSS correlated moderately with BIQ-Fear. A low correlation was found between DQ and BIQ-Fear, while the correlation between DS and BIQ-Fear was moderate. 


\section{DISGUST PROPENSITY AND DISGUST SENSITIVITY}

Correlations of DPSS, DQ, and DS with the FSQ were all comparable. To prevent artificially inflated correlations between DS and BIQ-Fear / FSQ, a DS total score without subscales Animals, Death and Body-Envelope Violations was used. Additionally, a separate DS total score containing only these subscales was calculated. Table 4 displays that correlations between the fear questionnaires and the DS containing overlapping items are indeed more pronounced compared to the DS without those subscales.

Table 4.

Correlations between disgust indices and fear questionnaires.

\begin{tabular}{lll}
\hline & FSQ & BIQ-Fear \\
\hline DPSS Propensity & $-.20^{* *}$ & $-.35^{* *}$ \\
DPSS Sensitivity & $-.16^{* *}$ & $-.35^{* *}$ \\
DQ & $-.24^{* *}$ & $-.26^{* *}$ \\
DS total & $-.17^{* *}$ & $-.42^{* *}$ \\
$\quad$ Overlap & $-.21^{* *}$ & $-.48^{* *}$ \\
$\quad$ No overlap & $-.10^{* *}$ & $-.26^{* *}$ \\
\hline Valid N & & 967 \\
\hline$* *$ Significant at $p<.01$ & &
\end{tabular}

To correct correlations between DPSS and FSQ / BIQ for variance already explained by the DQ and DS, partial correlations were calculated. Table 5 depicts that this leads to slightly lower, yet still significant correlations between DPSS and BIQ-Fear / FSQ. Thus, the DPSS has additional independent predictive value compared to the $\mathrm{DS}$ and $\mathrm{DQ}$.

Table 5.

Partial correlations, controlling for DQ and DS

\begin{tabular}{lll}
\hline & DPSS-Propensity & DPSS-Sensitivity \\
\hline FSQ & $.13^{* *}$ & $.10^{* *}$ \\
BIQ Fear & $.23^{* *}$ & $.26^{* *}$ \\
\hline Valid N & & 967 \\
\hline
\end{tabular}

** Significant at $p<.01$

\section{Contribution to psychopathology}

Regression analyses were used to examine whether propensity and sensitivity contribute independently to the variance in fear of blood and fear of spiders. Scores on the FSQ and BIQ were used as dependent variables, whereas scores on DPSS subscales were included as predictor variables. Of the variance in FSQ scores, only $4 \%$ was accounted for by the DPSS scores $\left(R=.20, R^{2}=.04 ; F(2,966)=20,71 ; p<.01\right)$. FSQ scores were predicted significantly by Propensity $(\beta=.16 ; t=4.09 ; p<.01)$ but not by Sensitivity $(\beta=.06 ; t=1.62 ; p=.10)$. A total of $16 \%$ of the variance of BIQ-Fear scores was accounted for by the scores on the DPSS $\left(R=.39, R^{2}=.16 ; F(2,962)=88.39 ; p<.01\right)$. Scores on BIQ-Fear were significantly predicted by Propensity $(\beta=.22 ; t=6.00 ; p<.01)$ as well as by Sensitivity $(\beta=.22 ; t=6.04$; $p<.01)$. 


\section{CHAPTER 3}

Next, it was examined if people with a fainting history endorsed the DPSS differently compared to non-fainters. Based on the scores on BIQ-Fainting, the dataset was divided into two groups: people who never fainted $(n=797)$, and people who have fainted at least once in response to blood-injection stimuli $(n=164)$. A 2 (Propensity, Sensitivity) $\times 2$ (fainters, non-fainters) ANOVA was performed. The between-groups factor Fainting history was significant $(F(1,959)$ $=9.55 ; p<.01)$, thus, fainters scored significantly higher on the DPSS compared to nonfainters. Interaction term DPSS-subscale $\times$ Fainting history was not significant $(F(1,959)=.45$; $p=.50$ ), so the overall response pattern did not differ for fainters and non-fainters. Subsequently, a series of contrast-specific one-way ANOVAs revealed that fainters scored significantly higher than non-fainters on propensity $(F(1,960)=4.86 ; p=.03)$ and sensitivity $(F(1,960)=11.86 ; p<.01)$.

\section{Discussion}

The main findings are: i) supporting its content validity, exploratory factor analysis revealed that the DPSS consists of two factors: propensity and sensitivity, ii) confirmatory factor analysis confirmed this two-factor model in an independent dataset, iii) the DPSS subscales have high internal consistency and acceptable test-retest reliability, iv) correlations between DPSS and other disgust indices (DS, DQ) were moderate, but comparable to correlations between DS and $D Q, v)$ disgust propensity and sensitivity correlated in the expected direction with spider and blood fear, vi) these correlations remain when correcting for variance already explained by DS and $D Q$, vii) disgust propensity was a significant predictor of spider fear, whereas both disgust propensity and sensitivity were significant predictors of fear of blood, viii) participants with a fainting history for BII-stimuli endorsed the DPSS more strongly than those without.

Supporting content validity of the DPSS, exploratory factor analysis revealed that the DPSS meaningfully differentiated between disgust propensity (the tendency to react with the emotion of disgust) and disgust sensitivity (the tendency to experience disgust as something 'horrid'). Moreover, confirmatory factor analysis confirmed the two subscales in an independent sample and showed it fitted the data significantly better than a one-factor solution.

Further, the DPSS correlated moderately with other disgust propensity questionnaires (DS, DQ). The propensity measures (DPSS-Propensity, DS, DQ) correlated low to moderate, suggesting that each measures different aspects of disgust propensity. These findings are similar to previously reported correlations between DS and DQ $(r=-.33$; de Jong, Peters, \& Vanderhallen, 2002; $r=-.54$; de Jong \& Merckelbach, 1998), and are in accordance with studies showing that whilst the DQ refers to core disgust (de Jong \& Merckelbach, 1998), the DS taps into several disgust domains (Haidt et al, 1994). Additionally, these findings support the idea that DPSS-Propensity measures domain-independent disgust experiences.

Correlations between disgust questionnaires (DPSS, DS, DQ) and measures of psychopathology (FSQ, BIQ) were similar to earlier studies on the relationship between disgust propensity and spider fear (e.g., DQ and Spider Phobia Questionnaire (SPQ): $r=-.35$, de Jong, Andrea, Muris, 1997; $r=-.27$, de Jong \& Merckelbach, 1998; DS and SPQ: $r=.18$, Sawchuk et 
al., 2000) or between disgust propensity and fear of BII-stimuli (DQ and five Fear Survey Schedule items: $r=.33$, Matchett \& Davey, 1991; DS and BIQ: $r=.42$; de Jong \& Merckelbach, 1998; DS and Injection Phobia Scale-Anxiety: $r=.32$, Sawchuk et al., 2000). In line with previous research (de Jong \& Merckelbach, 1998), the FSQ displayed the strongest association with the DQ (measuring core disgust), whereas the BIQ was more strongly associated with the DS (measuring core and animal-reminder disgust). Compared to spider fear, fear of blood (BIQ) was relatively strongly associated with disgust propensity (DS, DQ). These findings confirm studies that found disgust to be more strongly involved in blood phobia than in spider phobia (Sawchuk et al., 2000; 2002; Tolin et al., 1997).

For the DPSS, the same pattern of results was observed. Both subscales correlated more strongly with BIQ-Fear, yet comparably to the DQ with the FSQ. The finding that the correlation between disgust propensity and indices of psychopathology still holds when a questionnaire is used that does not refer to specific disgust elicitors is an important finding, because it indicates that the previously observed relationship between disgust propensity and psychopathology is not merely an artefact of conceptual overlap between questionnaires (e.g., Thorpe \& Salkovskis, 1998).

Interestingly, the DPSS-subscales appear differently associated with psychopathology: fear of spiders was most strongly associated with propensity, whereas fear of blood was related to both propensity and sensitivity. Therefore, the interplay between disgust propensity and sensitivity may differ across disorders. For example, although spider phobics consider spiders disgusting, feelings of fear tend to predominate (Tolin et al., 1997; Sawchuk et al., 2000; 2002). If fear is the dominant response, then high levels of disgust sensitivity may not be critically involved. In blood phobia, however, disgust appears the dominant emotion (Page, 1994, 2003; Tolin et al., 1997). Accordingly, high levels of disgust sensitivity are likely to play a more prominent role in fear of blood, since disgust sensitivity determines to what extent confrontation with BII- stimuli is being experienced as unpleasant.

Additionally, BII-fearful individuals are characterized by a unique symptom, the vasovagal syncope. Previous work indicates that disgust may be involved in BII-related fainting (Page, 1994, 2003). In accordance with this idea, people with a fainting history for BII-stimuli displayed significantly elevated levels of both disgust propensity and sensitivity, compared to non-fainters in the present study.

Several points should be addressed in future research. First, it seems warranted to establish validity of the DPSS in terms of predictive validity for individuals' actual behavioral tendency to avoid disgusting objects. To corroborate the relevance of differentiating between sensitivity and propensity, it would be important to explore the differential predictive validity of these factors. Second, divergent validity should be examined. For example, it would be recommendable to determine if the DPSS differs from related indices, such as the Anxiety Sensitivity Index (Reiss et al., 1986). Third, test-retest reliability was lower than expected. One testable explanation may be that the time interval between measurements was quite long (two months). Further, although confirmatory factor analysis revealed that the two-factor structure was superior to the one-factor model, correlations between propensity and sensitivity were high. More studies are needed to establish if propensity and sensitivity are indeed to be regarded as separate traits. 


\section{CHAPTER 3}

Additionally, the majority of participants in this study were women. Therefore, it remains to be determined whether psychometric properties of the DPSS are similar in samples in which the numbers of men and women are balanced. Finally, although the present study indicates that disgust propensity and sensitivity are differentially related to fear of spiders and fear of blood, it needs to be examined further whether these findings are similar when a treatment-seeking sample is used. 


\section{Chapter 4}

The Disgust Propensity and Sensitivity Scale - Revised:

Its predictive value for avoidance behavior and physiological responsivity

'Can't believe, disgust, disgust Can't believe, it scares me, Can't believe, can't believe' Slowdive 


\title{
CHAPTER 4
}

\begin{abstract}
There is ample evidence that disgust propensity is involved in various forms of psychopathology. However, current disgust propensity indices may display inflated correlations with indices of psychopathology due to conceptual overlap. The recently developed Disgust Propensity and Sensitivity Scale-Revised (DPSS-R) is unique in that it measures disgust propensity irrespective of specific elicitors. Although psychometric research has confirmed its factor structure, its predictive validity remains to be established. The major aim of the present study is therefore to test its predictive validity for disgust-induced avoidance behavior and disgust-induced psychophysiological reactivity. Hereto, participants $(N=60)$ completed the DPSS-R (van Overveld, de Jong, Peters, Cavanagh, \& Davey, 2006), engaged in seventeen behavioral experiments, and viewed a disgust-inducing video. Attesting to its predictive validity, disgust propensity as indexed by DPSS-RP was associated with completing fewer steps on the BATs. The video elicited self-reported disgust, disgust-specific muscular activity of the levator, and an increase in HF power indicating an increase in parasympathic activity. Yet, disgust propensity and disgust sensitivity did not correlate with psychophysiological reactivity during the disgust video. Thus, the DPSS-R was found to be a valid instrument for predicting disgustinduced avoidance behavior but not for physiological responding towards disgusting stimuli.
\end{abstract}


Accumulating evidence suggests that disgust propensity, as defined by the tendency to experience disgust more readily, may be involved in various psychopathological disorders, such as spider phobia (Matchett \& Davey, 1991), blood phobia (Page, 1994), eating disorders (Troop, Murphy, Bramon, \& Treasure, 2000), obsessive compulsive disorders (Olatunji, Sawchuk, Lohr, \& de Jong, 2004) and sexual dysfunctions (de Jong, van Overveld, WeijmarSchultz, Peters, \& Buwalda, in press).

However, the association between disgust propensity and specific clinical complaints may present an artifact in the sense that the current indices of disgust propensity contain conceptual overlap with indices of psychopathology. All of the most widely used disgust propensity scales such as the Disgust Scale (DS; Haidt et al., 1994), the Disgust Questionnaire (DQ; Rozin, Fallon, \& Mandell, 1984), and the Disgust Emotion Scale (DES; Walls \& Kleinknecht, 1996) assess disgust propensity in relation to specific objects, thereby possibly inflating the relationship between these scales and scales measuring psychopathology as both may refer to similar items (e.g., small animals).

To overcome this potential confound, the Disgust Propensity and Sensitivity Scale (DPSS; Cavanagh \& Davey, 2000) was designed. The DPSS assesses disgust propensity irrespective of specific disgust elicitors. Moreover, the DPSS was not only designed to measure disgust propensity but also to measure disgust sensitivity. In the context of psychopathology, it may not only be important how readily people respond with the emotion of disgust (disgust propensity), but also how unpleasant people consider the experience of disgust (disgust sensitivity). High levels of disgust propensity are assumed to enlarge the probability of particular stimuli to acquire a disgust-evoking status, whereas elevated levels of disgust sensitivity are assumed to motivate people to avoid certain situations at the prospect of experiencing the unpleasantly evaluated emotion of disgust. Thus, for disorders in which the emotion of disgust appears critically involved when confronted with phobic stimuli (e.g., blood phobia; Sawchuk, Lohr, Tolin, Lee, \& Kleinknecht, 2000), it might well be that in addition to disgust propensity, elevated levels of disgust sensitivity contribute to the phobic complaints (see e.g., van Overveld, de Jong, Peters, van Hout, Bouman, 2008). For example, previous research found that emetophobic individuals report to avoid aversive feelings of nausea (Boschen, 2007). In a recent study, the DPSS was validated and revised to a shorter version. This revised DPSS-R demonstrated good psychometric qualities in terms of internal consistency and factor structure (van Overveld, de Jong, Peters, Cavanagh, \& Davey, 2006). A validation study in an independent sample confirmed that the DPSS-R indeed contained two factors measuring disgust propensity and disgust sensitivity, and appeared psychometrically sound (Olatunji, Cisler, Deacon, Connolly, \& Lohr, 2007). An important next step would be to test the predictive validity of the DPSS-R. Therefore, the main aim of the present experiment was to investigate the predictive validity of the DPSS-R for both actual avoidance behavior (cf. Rozin, Haidt, McCauley, Dunlop, \& Ashmore, 1999) and disgust-induced psychophysiological responsivity.

The present study consists of two parts. In the first part, the DPSS-R was studied in relation to psychophysiological responsitivity during a disgust-inducing film. The emotion of disgust has been associated with heightened activity in the parasympathetic branch of the autonomic nervous system (Levenson, 1992). Accordingly, enhanced levels of disgust propensity were 


\section{CHAPTER 4}

accompanied by greater saliva production upon confrontation with disgusting stimuli (van Overveld, de Jong, \& Peters, submitted). Research also noted that experimentally induced disgust was accompanied by heightened reactivity of the disgust-specific $\mathrm{m}$. levator labii (de Jong, Peters, \& Vanderhallen, 2002; Yartz \& Hawk, 2002) as well as increases on more general arousal parameters like m. corrugator activity (de Jong, Peters, \& Vanderhallen, 2002), heart rate (Alaoui-Ismaïli, Robin, Rada, Dittmar, \& Vernet-Maury, 1997), and skin conductance level (Palomba, Sarlo, Angrilli, Mini, \& Stegagno, 2000). Following on from this, we anticipated that high levels of disgust propensity as indexed by DPSS-RP would be predictive of the intensity of disgust-related physiological responding during the disgust video. Since disgust sensitivity is intrinsically subjective, no straightforward predictions were made with respect to the relationship between disgust sensitivity and disgust-related physiological responding.

In the second part, the DPSS-R subscales were studied in relation to the willingness to engage in a series of disgust-inducing BATs. Additionally, self-reported state disgust was examined in relation to trait disgust. Earlier studies showed that high disgust propensity as indexed by the DS was strongly associated with the performance on a series of contaminationbased disgust BATs (Deacon \& Olatunji, 2007). In a similar vein, blood phobic individuals with enhanced levels of disgust propensity (DES) were less compliant to fully complete a series of disgusting behavioral experiments (e.g., touching a bloody gauze; Koch, O'Neill, Sawchuk, \& Connolly, 2002) than non-phobic participant. Accordingly, we anticipated that elevated levels of disgust propensity (as indexed by the DPSS-RP) would be associated with stronger behavioral avoidance as well as with increased levels of state disgust. Finally, because people who consider the experience of disgust as highly unpleasant will be less likely to engage in potentially disgust-evoking behaviors (e.g., Koch et al., 2002) we anticipated that not only high levels of disgust propensity but also of disgust sensitivity (DPSS-RS) would be predictive of less approach behavior in the context of disgust-inducing BATs.

\section{Methods}

\section{Participants}

A large sample of students from the faculties of Health Sciences, Medicine and Psychology at Maastricht University were asked to complete the DPSS-R $(N=216)$ and to indicate whether they would be willing to participate in a follow-up experiment. From this sample, sixty participants were approached and invited to the lab based on their scores of the DPSS-R in order to ensure that scores across a broad range of the DPSS-R would be included. Hereto, the initial research population ( $N=216)$ was divided into deciles, and it was attempted to include an equal number of participants per decile. As female students are overrepresented at these faculties, the current research population consists mostly of female participants (52 women; $86.7 \%$ ). The mean age of the research population was 21.58 years $(S D=2.95$; range: $18-39$ years). 


\section{Instruments}

Disgust Propensity and Sensitivity Scale-Revised (DPSS-R; van Overveld et al, 2006). This 16item questionnaire measures the constructs of disgust propensity and sensitivity. Participants read a series of propositions on the frequency of experiencing bodily sensations and their emotional impact, and have to indicate which applies best to them on a scale from 1 (= 'never') to 5 (= 'always'; range: 16-80). Previous work showed that the DPSS-R was internally consistent, with alpha coefficients of .78 (Propensity) and .79 (Sensitivity) (van Overveld, de Jong, Peters, Cavanagh \& Davey, 2006).

Disgust Emotion Scale (DES; Walls \& Kleinknecht, 1996); The DES examines disgust propensity for five types of disgust elicitors: Animals (DES-An), Mutilation and Death (DES-Mut), Injections and Blood Draws (DES-Inj), Rotting Foods (DES-Rot), and Smells (DES-Sm). The DES consists of 30 items on which participants rate how much disgust they would experience if they were to be confronted with a certain object (e.g., the smell of vomit) on a five point Likert-type scale (from $0=$ 'No disgust or repugnance at all' to $4=$ 'Extreme disgust or repugnance'). The DES and its subscales are internally consistent (all a's > .73; Sawchuk, Lohr, Westendorf, Meunier, \& Tolin, 2002).

Disgust and Contamination Sensitivity Questionnaire (DQ; Rozin, Fallon, \& Mandell, 1984); This instrument measures disgust propensity for core disgust. It assesses the tendency to reject desirable and edible food items that have become contaminated by certain disgusting stimuli. Participants indicate on a scale from 1-9 (1 = 'not at all', 9 = 'very much') how much they would prefer to eat these contaminated food items. The scores can then be summed up to calculate a total score (range 24-216). Internal consistency is good (alpha $=.72$ to .86 , Davey, 1994; .80, Mulkens, de Jong, \& Merckelbach, 1996).

Disgust Scale (DS; Haidt et al., 1994). The DS is a 32-item questionnaire, measuring disgust propensity for eight subscales of disgust elicitors: Body-Envelope Violations, Animals, Bodily Products, Hygiene, Sex, Food, Death and Sympathetic Magic. The DS consists of two parts. On the first part, participants rate if they agree with a series of 16 propositions using a dichotomous scoring format ( $0=$ 'false', $1=$ 'true'). On the second part, participants indicate how much they are disgusted by 16 objects or situations on a scale from 0 (= 'not disgusting at all') to 2 (= 'extremely disgusting'). The total score on the DS is a reliable index for disgust propensity (alpha $=.84$; Haidt et al., 1994).

Visual Analog Scale (VAS). Participants rated on three $100 \mathrm{~mm}$ VAS's the state levels of disgust (how much disgust did you experience during this last step?), horridness (how horrid did you find this last step?), and happiness (how happy were you during this last step?) that they had experienced during each step of each BAT.

\section{Apparatus}

Swallowing; The amount of excreted saliva can be interpreted as an index of parasympathetic activity (Proctor \& Carpenter, 2007). In the present study, saliva excretion was measured by continuous measurement of the EMG activity of the $\mathrm{m}$. digastricus by using two pairs of $\mathrm{Ag} / \mathrm{AgCl}$ 


\section{CHAPTER 4}

Beckman electrodes (diameter $2.5 \mathrm{~mm}$ ) filled with Spectra 360 electrode gel. The indirect measurement of saliva production via EMG has been shown to be a sensitive and valid instrument (Nederkoorn, Smulders, \& Jansen, 1999).

Facial ElectroMyography (EMG); In addition to the m. digastricus, EMG activity was measured for two facial muscles, i.e., the m. corrugator (as an index for general arousal) and the $\mathrm{m}$. levator labii (as an index for disgust). Measurements were obtained using two pairs of $\mathrm{Ag} / \mathrm{AgCl}$ Beckman electrodes (diameter $2.5 \mathrm{~mm}$ ) that were filled with Spectra 360 electrode gel. Following recommendations by Fridlund and Cacioppo (1986), all electrodes were placed on the left side of the face.

ElectroCardioGram (ECG); The ECG recording were made by placing pre-gelled single-use disposable ECG surface electrodes (Ambu Bluesensor). Two electrodes were placed on the participant's lower ribs, and one on the sternum. With three unipolar leads, the electrodes were connected directly with a Picker/Schwarzer Coupler (ED14/12). The ECG signals were monitored continuously and saved on the lab server for off-line processing. Respiration rate was not included in the present study.

Skin conductance level (SCL); Using a Schwarzer EDA48 Amplifier, skin conductance was measured using the method of constant voltage (.5 volts). Two $\mathrm{Ag} / \mathrm{AgCl}$ electrodes (diameter $7.0 \mathrm{~mm}$ ) were placed at the palmar side of the middle phalanges of the second and third fingers of the participant's non-dominant hand. The electrodes were filled with isotonic gel.

All ECG and EMG signals were fed through a Picker/Schwarzer Coupler (ED14/12) to a PXI data-acquisition system (PXI 4472; National Instruments) with a sample frequency of $1000 \mathrm{~Hz}$ with a time constant of $0.3 \mathrm{~s}$. Additionally, a notch filter was used to eliminate any $50 \mathrm{~Hz}$ interference. Further, a low pass filter of $1.60 \mathrm{~Hz}$ and a high pass filter of $300 \mathrm{~Hz}$ were used. For skin conductance, signals were fed to the PXI-data acquisition system through a $500 \mathrm{~Hz}$ anti-aliasing filter. Data was stored on the lab server and processed off-line.

\section{Materials}

Film clips, prior to the start of the behavioral experiments, three five-minute film clips were presented: a neutral film (a documentary by Bert Haanstra on making glass), a disgusting movie (excerpts from MTV's Jackass, containing an egg-eating contest and a milk-drinking contest, during which participants vomit excessively) and a second neutral film (the second part of the documentary of making glass). The movies were presented on a 17 inch computer screen, approximately two meters from the individual. The movies were stored as avi files on the lab computer (Dell GX270, Pentium 4A, 3GHz, Windows 2000). A comparison between the first baseline measurement and the disgust inducing films was used to determine disgustrelated physiological responsivity, the last baseline was used to wear off the effects of the disgust induction.

Behavioral Approach Tests; A total of 17 separate BATs were devised, more specifically, one neutral BAT (0. Stirring water with a finger) and sixteen disgust-related experiments. Four tests were designed for the four domains of disgust which are generally accepted in disgust research (Rozin, Haidt, \& McCauley, 1999; Woody \& Teachman, 2000): Core disgust (1. Drinking from a 
dog's bowl, 2. Taking a bite from a cookie placed in a cookie jar that contained dead grasshoppers, 3. Touching a live worm with one's lips, 4. Drinking juice from a glass in which an ice cube containing a dead grasshopper has been dipped several times.), Animal-reminder disgust (A-R; 5. Touching a dead, stuffed spider crab, 6. Sticking a needle in an actual pig's head, 7. Touching a band aid that contained a spot that looked like blood and of which participants were told it was sterilized blood, but which was actually iodine, 8. Sticking a needle in a severed cow's leg), Interpersonal disgust (IP; 9. Wiping participant's face with clearly used and unwashed towels, 10. Having to comb one's hair with a clearly used hair brush, 11. Sticking an ECG-electrode of the previous participants on one's arm, 12. Stirring a finger through a glass of water that, as participants were told, contained spit of the previous participant. It actually did not contain anything), and Socio-moral disgust (SM; 13. Viewing a movie scene from American History $X$, where a group of neo-Nazi's rob a supermarket, as well as taunt and humiliate the African American shopkeepers, 14. Viewing a movie scene from Happiness, where a man harasses a woman via an obscene phone call. At first, the woman is unaware, but she soon discovers that his questions and requests are very explicit. Meanwhile, the man masturbates and ejaculates at the end of the movie clip, 15. Eating a slice of horse meat, 16. Sticking a needle through a doll's eye). Although some tests were derived from previous research (Rozin et al., 1999), most were devised by the current authors. The order in which the tests were administered were randomized and then given a fixed order. Thus, the order was the same for all participants $(0,16,7,13,4,15,1,6,11,12,10,14,3,5,9,2,8)$.

\section{Procedure}

Upon arrival at the lab, participants were asked to complete a packet of questionnaires containing Dutch versions of the DPSS-R, DS, DQ, and DES. Next, they received a short introduction on the two parts of the experiment and informed consent was obtained. Following this, electrodes were placed and the physiological signals were tested. In the first part of the experiment, participants viewed a neutral film, a disgusting film, and a second neutral film. After each film, participants completed the three VASs. In the second part of the experiment, participants engaged in a series of disgust-inducing behavioral experiments. Following each step of the BATs, the participants completed the VAS-scales. At the end of the experiment that lasted about an hour, participants were debriefed and received ten euros for their participation.

\section{Data reduction}

For the EMG data, specifically designed software was used to digitally rectify and integrate the signals with a time constant of $0.3 \mathrm{~s}$. Following this, a root transformation was applied to normalize the skewed distribution of the facial EMG data. Then, mean EMG activity for the facial EMG (i.e., the m. corrugator, m. levator, and m. digastricus) was calculated. For heart rate, specifically designed software was used to determine the R-tops in the ECG data. Using the interval between these successive R-tops, the heart rate was then calculated. 


\section{CHAPTER 4}

Heart rate variability was defined as the variability of the intervals between successive heart beats (R-tops in the ECG). Generally, three components can be established: low frequency, middle frequency and high frequency. As the high frequency component is under the influence of the nervus vagus, the power in this High Frequency band (HF: $0.15-.40 \mathrm{~Hz}$ ) is assumed to indicate parasympathetic activity (Task Force of the European Society of Cardiology and the North American Society of Pacing Electrophysiology, 1996). Consequently, only the power in the High Frequency band was calculated in this experiment. To calculate the power in the HF-band, the CARSPAN software package (IEC ProGamma, Groningen, the Netherlands) was used, which conducted a spectral analysis on the Inter-Beat Intervals according to a discrete Fourier Transform algorithm. As suggested by the Task Force of the European Society of Cardiology and the North American Society of Pacing Electrophysiology (1996), the normalized units were then calculated for the power in the HF-band for each film clip by using the following formula: HF power / (total power - VLF) x 100.

Next, the T-wave amplitude was obtained by calculating the difference in $\mu \mathrm{V}$ between the maximum value included in a 100-300 ms window following the R-wave and the mean value of the 40-ms isoelectric line between the P-wave and Q-wave (Rau, 1991). Following this, the mean T-wave amplitude during the films was calculated. The T-wave amplitude can be used as an indicator for withdrawal of sympathetic activity (Kline et al., 1998).

For skin conductance level, the mean activity during each test was calculated. For the BATs, the number of total steps completed was calculated (range 0-51) as well as the mean number of steps completed during the four BATs that comprise each disgust domain (range 0-3). For state disgust, horridness, and happiness, mean VAS-scores of the first step were calculated per domain (Core, A-R, IP, SM) and overall. The first steps of each BAT were used since all participants engaged in these steps. Further, not only was the data complete for these steps, scoring on the first step was independent of the total steps that participants completed during the BATs.

Then, using the Statistical Package for Social Sciences (SPSS version 11.0), missing value analyses were conducted for the questionnaire data, using SPSS' missing value analysis. This analysis computes missing data via regression analyses.

\section{Results}

\section{Descriptives}

In Table 1, the means and standard deviations for all questionnaires and the BAT scores are presented for all participants. Additionally, the Cronbach's alpha of all questionnaires and the BAT-scores are presented. With the exception of the DS subscales and total score, all questionnaires and the BATs appeared internally consistent. 
Table 1.

Descriptive statistics for all indices of disgust propensity (DPSS-RP, DES, DS, and DQ) and disgust sensitivity (DPSSRS) and BAT-scores for all participants.

\begin{tabular}{lcccl}
\hline Indices & Means $(n=60)$ & SD & range (min - max $)$ & a \\
\hline DPSS-RP & 20.61 & 3.70 & $12-28(8-40)$ & .69 \\
DPSS-RS & 16.10 & 4.16 & $9-26(8-40)$ & .79 \\
DES total & 36.95 & 17.56 & $8-86(0-120)$ & .92 \\
DS total & 16.37 & 2.40 & $12-23(0-32)$ & .61 \\
DQ total & 140.68 & 30.48 & $69-207(24-216)$ & .94 \\
BAT steps taken & 45.53 & 5.06 & $26-51(0-51)$ & .82 \\
Core & 2.58 & .33 & $1.25-3(0-3)$ & .65 \\
A-R & 2.70 & .57 & $.5-3(0-3)$ & .78 \\
IP & 2.60 & .44 & $1.25-3(0-3)$ & .73 \\
SM & 2.76 & .40 & $1-3(0-3)$ & .60 \\
\hline Valid $N$ & & & & 60
\end{tabular}

Abbreviations: DPSS-RP = Disgust Propensity and Sensitivity Scale - Revised Propensity, DPSS-RS = Disgust Propensity and Sensitivity Scale - Revised Sensitivity, DES = Disgust Emotion Scale, DS =Disgust Scale, DQ = Disgust Questionnaire, BAT = Behavioral Approach Task, AR = Animal-reminder disgust, IP = InterPersonal disgust, $\mathrm{SM}=$ SocioMoral disgust. * Significant at $\mathrm{p}<.05 ; * *$ Significant at $\mathrm{p}<.01$

\section{Subjective and psychophysiological responding during disgust video}

The physiological activity during the disgust film was compared to the activity during the first baseline. Table 2 shows the differences in physiological and subjective reactivity between the neutral and the disgust film. Attesting to the validity of the present manipulation, the levels of state disgust (VASs) were significantly higher during the disgust film compared to the neutral film. In addition, state happiness was significantly lower during the disgust film.

Table 2.

Physiological and subjective reactivity during neutral film and disgusting film

\begin{tabular}{|c|c|c|c|c|}
\hline Parameters & Neutral film & Disgust film & $t(60)$ & $p$ \\
\hline & $7.25(3.91)$ & $7.61(4.30)$ & -2.40 & .02 \\
\hline \multicolumn{5}{|l|}{ Facial EMG } \\
\hline Corr & $8.11(4.50)$ & $7.58(4.29)$ & 1.72 & .09 \\
\hline Lev & $6.23(3.18)$ & $8.51(5.46)$ & -5.21 & $<.01$ \\
\hline \multicolumn{5}{|c|}{ Cardiovascular parameters } \\
\hline $\mathrm{HR}$ & $78.45(11.14)$ & $76.35(11.80)$ & 2.10 & .04 \\
\hline $\mathrm{HF}$ & $56.21(49.29)$ & $81.45(79.46)$ & -1.92 & .06 \\
\hline TWA & $275.18(134.53)$ & $267.84(128.48)$ & 2.14 & .04 \\
\hline $\mathrm{SCL}$ & $1.50(.86)$ & $2.01(1.01)$ & -8.23 & $<.01$ \\
\hline \multicolumn{5}{|c|}{ Self report indices } \\
\hline ED & $1.63(5.51)$ & $49.85(30.21)$ & -12.35 & $<.01$ \\
\hline $\mathrm{EH}$ & $1.87(4.00)$ & $27.80(28.18)$ & -6.93 & $<.01$ \\
\hline EHA & $53.20(18.61)$ & $24.55(27.77)$ & 2.11 & .04 \\
\hline
\end{tabular}

Abbreviations: Dig = Digastricus, Corr $=$ Corrugator, $\mathrm{Lev}=$ Levator, $\mathrm{HR}=$ Heart rate, $\mathrm{HF}=$ Power in High Frequency band of heart rate variability, TWA = T-wave amplitude, $\mathrm{SCL}=$ Skin conductance level, ED = Experienced Disgust, $\mathrm{EH}=$ Experienced Horridness, $\mathrm{EHA}=$ Experienced Happiness. 


\section{CHAPTER 4}

As expected, a significant increase in activity was observed in the digastricus and the levator labii. Moreover, a border-significant decrease in activity of the $\mathrm{m}$. corrugator suggests that the reactivity in facial EMG was disgust-specific. Heart rate decreased and there was a bordersignificant increase of the power in the HF band. Both responses are consistent with an increase in parasympathetic activity. Additionally, there was a significant decrease in T-wave amplitude indicating that there was (also) an increase in sympathetic activity during the disgust video. As expected, a significant increase in SCL suggests that the disgust video elicited generally more arousal than the neutral video.

\section{Correlations between state and trait disgust and physiological reactivity}

To test whether trait disgust propensity (DPSS-RP) and disgust sensitivity (DPSS-RS) correlated with state disgust (mean VAS-scores during disgust-film minus VAS-scores during neutral film) as well as with physiological reactivity (mean physiological activity during disgust-film minus mean activity during neutral film), a series of bivariate Pearson correlations were conducted. To test the predictive value of the DPSS-R compared to other indices for disgust propensity, correlations were examined for all questionnaires (DES, DS, DQ). As Table 3 shows, the trait indices DPSS-RP and DPSS-RS did not correlate significantly with physiological reactivity. Both scales did not correlate significantly with experienced disgust (both $p$ 's $>.14$ ), whereas unexpectedly both scales did correlate with experienced horridness $(p<.05)$. Unexpectedly, experienced disgust and horridness did not correlate significantly with physiological reactivity, with only two exceptions. Experienced horridness correlated significantly with corrugator activity $(r=.33 ; p<.05)$ and HF power $(r=.26 ; p<.05)$.

\section{Table 3.}

Bivariate correlations between the disgust indices (DPSS-R, DES, DS, DQ, and physiological responsivity (mean activity during disgust-film minus mean activity during baseline film).

\begin{tabular}{llllll}
\hline Indices & DPSS-RP & DPSS-RS & DES total & DS total & DQ \\
\hline Dig & -.18 & -.09 & -.21 & .05 & .15 \\
Corr & -.19 & -.11 & -.08 & -.18 & -.12 \\
Lev & .11 & .03 & .17 & $.34 * *$ & -.13 \\
HR & .05 & -.12 & .12 & -.00 & .08 \\
HF & -.08 & .04 & $.27 *$ & $.28 *$ & -.08 \\
TWA & -.20 & .20 & -.08 & .05 & -.22 \\
SCL & .19 & .17 & -.04 & .01 & -.07 \\
ED & .19 & .19 & $.39 * *$ & $.43 * *$ & -.20 \\
EH & $.31^{*}$ & $.33 *$ & $.33^{*}$ & $.39 * *$ & -.04 \\
\hline Valid $N$ & & & & & 60
\end{tabular}

Abbreviations: DPSS-R = Disgust Propensity and Sensitivity Scale - Revised, DPSS-RP = Disgust Propensity, DPSS-RS $=$ Disgust Sensitivity, DES = Disgust Emotion Scale, DS =Disgust Scale, DQ = Disgust Questionnaire, ED = Experienced Disgust, $\mathrm{EH}=$ Experienced Horridness, $\mathrm{Dig}=$ Digastricus, Corr $=$ Corrugator, $\mathrm{Lev}=$ Levator, $\mathrm{HR}=\mathrm{Heart}$ rate, $\mathrm{HF}=$ Power in High Frequency band of heart rate variability, TWA = T-wave amplitude, SCL $=\mathrm{Skin}$ conductance level. * Significant at $\mathrm{p}<.05, * *$ Significant at $\mathrm{p}<.01$ 
Nevertheless, the correlations were relatively small. Thus, it appears that physiological reactivity and subjective experience present two largely dissociated phenomena. Linear regression analysis with experienced horridness as dependent variable and the two DPSS-R subscales as predictors showed that neither disgust propensity $(\beta=1.36 ; t=1.12 ; p=.27)$, nor disgust sensitivity $(\beta=1.58 ; t=1.47 ; p=.15)$ did contribute independently to state horridness.

In contrast to the DPSS-R, the DS and the DES correlated significantly with the power in the HF band. The DS scores were also associated with the levator activity during the disgust film. Finally, the DS and DES also correlated with the subjective indices of state disgust.

\section{Correlations between state and trait disgust and disgust avoidance}

As can be seen in Table 4, DPSS-RP had predictive value for the number of steps completed during the BATs. The predictive value of the DPSS-RP was comparable to that of the more widely used instruments for disgust propensity (i.e., DS, DQ, DES). Disgust sensitivity (as indexed by DPSS-RS) did not correlate significantly with the number of completed steps on the BATs $(p=.14)$, but it did correlate significantly with performance on the core BATs $(p<.05)$ and border-significantly with the A-R BATs $(p=.07)$. As to be expected, the DQ (index for Core disgust propensity) correlated most strongly with the number of steps taken during the Core BATs, together with DPSS-RP. Next, the number of steps during A-R BATs correlated best with the DPSS-R, DES and DS. The number of steps during IP BATs correlated most strongly with all disgust propensity indices with the exception of the DPSS-R, although the correlation was in the expected direction for DPSS-RP.

Moreover, none of the disgust indices correlated with the number of steps during the SM BATs. Since the internal consistency of these BATs was also low (alpha $=.60$ ), this suggests that these BATs may not have been as successful in inducing (sociomoral) disgust as the other disgust BATs.

Table 4.

Bivariate correlations between disgust propensity (DPSS-RP, DES, DS, DQ), disgust sensitivity (DPSS-RS), and the number of BAT-steps taken.

\begin{tabular}{llllll}
\hline Index & Core & A-R & IP & SM & Total steps \\
\hline DPSS-RP & $-.39^{*}$ & $-.30^{*}$ & -.21 & .01 & $-.31^{*}$ \\
DPSS-RS & $-.26^{*}$ & $-.24^{\mathrm{a}}$ & -.13 & .09 & -.19 \\
DES total & -.07 & $-.25^{*}$ & $-.34^{* *}$ & -.16 & $-.30^{*}$ \\
DS total & -.10 & $-.25^{*}$ & $-.36^{* *}$ & -.18 & $-.32^{*}$ \\
DQ & $.42^{* *}$ & .13 & $.34^{* *}$ & -.04 & $.28^{*}$ \\
\hline Valid N & & & & 60
\end{tabular}

Abbreviations: Core $=$ Core disgust BATs, AR = Animal-reminder disgust BATs, IP = InterPersonal disgust BATs, SM $=$ SocioMoral disgust BATs, DPSS-RP = Disgust Propensity and Sensitivity Scale-Revised Propensity, DPSS-RS = Disgust Propensity and Sensitivity Scale-Revised Sensitivity, DS-total = Disgust Scale, DES = Disgust Emotion Scale, $\mathrm{DQ}=$ Disgust Questionnaire. ${ }^{*}$ Significant at $\mathrm{p}<.05 ; * *$ Significant at $\mathrm{p}<.01$; ${ }^{\text {a }}$ Significant at $\mathrm{p}<.10$ 


\section{CHAPTER 4}

\section{Additional predictive value of the DPSS- $R$}

To determine the relative strength of the predictive value of the DPSS-R subscales compared to the other indices for disgust propensity (DES, DQ, DS), a backward regression analysis was conducted with the number of steps taken as independent variable. As Table 5 shows, only disgust propensity indices DS $(\beta=-.62 ; t=-2.42 ; p=.02)$ and DPSS-RP $(\beta=-.33 ; t=-1.98$; $p=.05$ ) remained in the final equation indicating that the DS and DPSS-R have independent predictive value for the number of total BAT-steps taken.

Table 5.

Backward regression analyses to examine the relative predictive value of the DPSS-R subscales for total number of steps taken during the BATs.

\begin{tabular}{llccc}
\hline Questionnaire & $B$ & $S E B$ & $B$ & $p$ \\
\hline & & & & \\
Total steps & Step 1 & $R^{2}=0.22, F=2.93, p=0.02$ & & .08 \\
DPSS-RP & -.36 & .21 & -.27 & .09 \\
DPSS-RS & .11 & .19 & -.09 & .55 \\
DES total & -.02 & .04 & -.24 & .08 \\
DS total & -.51 & .29 & .17 & .19 \\
DQ & .03 & .02 & & \\
& & & & .05 \\
DPSS-RP & Final & $R^{2}=0.18, F=6.14, p<0.01$ & -.25 & .02 \\
DS total & -.33 & .17 & -.30 & 60 \\
\hline Valid N & -.62 & .26 & &
\end{tabular}

Abbreviations: DPSS-RP = Disgust Propensity and Sensitivity Scale-Revised Propensity, DPSS-RS = Disgust Propensity and Sensitivity Scale-Revised Sensitivity, DES = Disgust Emotion Scale, DS total = Disgust Scale, DQ = Disgust Questionnaire.

\section{State disgust during BATs}

To ensure that the BATs were successful in eliciting disgust, participants' mean levels of state disgust and horridness were calculated for both the BATs overall and per domain. Table 6 presents the means of experienced disgust, horridness, and happiness of the first step of each BAT, as well as the mean score per domain (Core, A-R, IP, SM).

A series of paired sample t-tests revealed that compared to the first step of the neutral BAT, a significant increase was observed during the first step of all BATs for experienced disgust (all $p$ 's $<.01$ except the needle doll test: $t=-1.78 ; p=.08)$ and horridness $(p<.03)$. Additionally, participants' happiness decreased compared to the neutral test (all $p$ 's $<.01$ ) with the exception of the needle doll test $(t=.78 ; p=.43)$, the dog's bowl $(t=.53 ; p=.59)$ and the electrode $(t=1.68 ; p=.09)$. 
Table 6.

Mean levels of state disgust, horridness, and happiness during the first step of each disgust-BAT and the means per domain.

\begin{tabular}{|c|c|c|c|c|c|c|}
\hline & ED & Actual Range & $\mathrm{EH}$ & Actual Range & EHA & Actual Range \\
\hline Neutral (water) & $.55(1.33)$ & $0-7$ & $.72(1.71)$ & $0-8$ & $48.75(22.70)$ & $0-100$ \\
\hline Core & 7.54 (9.47) & $0-43$ & $6.97(8.23)$ & $0-45$ & $43.42(22.73)$ & $0-100$ \\
\hline Ice cube & $8.17(13.87)$ & $0-65$ & $7.80(11.25)$ & $0-55$ & $41.88(22.77)$ & $0-100$ \\
\hline Dog's bowl & $1.42(3.42)$ & $0-19$ & $1.73(3.76)$ & $0-18$ & $47.88(23.66)$ & $0-100$ \\
\hline Worm & $10.85(17.20)$ & $0-80$ & 11.35 (16.44) & $0-84$ & $41.98(23.76)$ & $0-100$ \\
\hline Cookie & $9.75(15.20)$ & $0-64$ & $7.00(11.07)$ & $0-52$ & $41.93(25.68)$ & $0-100$ \\
\hline$A-R$ & $14.15(14.54)$ & $0-68$ & $15.24(15.64)$ & $0-70$ & $40.74(22.74)$ & $0-100$ \\
\hline Band aid & $7.33(10.22)$ & $0-53$ & $6.87(9.62)$ & $0-42$ & $42.23(23.84)$ & $0-100$ \\
\hline Pig's head & $21.57(21.90)$ & $0-85$ & $23.70(23.12)$ & $0-90$ & $38.42(24.50)$ & $0-100$ \\
\hline Spider crab & $9.05(16.23)$ & $0-84$ & $12.73(18.84)$ & $0-83$ & $43.23(23.31)$ & $0-100$ \\
\hline Horse's leg & $18.65(22.93)$ & $0-87$ & $17.67(21.70)$ & $0-88$ & 39.08 (24.93) & $0-100$ \\
\hline IP & $7.24(8.41)$ & $0-51$ & 6.47 (9.45) & $0-49$ & 43.58 (23.59) & $0-100$ \\
\hline Electrode & $2.68(5.10)$ & $0-27$ & 2.98 (5.75) & $0-26$ & $45.90(23.34)$ & $0-100$ \\
\hline Saliva & $6.27(10.38)$ & $0-66$ & $5.13(9.97)$ & $0-58$ & $43.20(23.94)$ & $0-100$ \\
\hline Hair brush & $7.52(12.46)$ & $0-48$ & $6.22(12.62)$ & $0-76$ & $42.67(24.62)$ & $0-100$ \\
\hline Dirty towel & $12.50(15.23)$ & $0-65$ & $11.53(16.20)$ & $0-66$ & $42.57(24.75)$ & $0-100$ \\
\hline SM & $11.77(11.16)$ & $0-41$ & $11.44(11.72)$ & $0-41$ & 39.99 (22.76) & $0-100$ \\
\hline Needle doll & $.78(1.54)$ & $0-6$ & $1.47(3.53)$ & $0-20$ & $48.03(22.53)$ & $0-100$ \\
\hline Robbery film & $17.92(21.52)$ & $0-81$ & $22.33(25.29)$ & $0-91$ & 32.65 (25.38) & $0-100$ \\
\hline Horse meat & $4.33(8.60)$ & $0-55$ & $5.20(9.20)$ & $0-37$ & $43.25(24.07)$ & $0-100$ \\
\hline Phonecall film & $24.05(24.98)$ & $0-93$ & $16.77(20.87)$ & $0-73$ & $36.03(25.62)$ & $0-100$ \\
\hline Total overall & $9.61(8.33)$ & $0-38$ & 9.48 (9.09) & $0-40$ & $42.33(22.31)$ & $0-100$ \\
\hline Valid $\mathrm{N}$ & & & & & & 60 \\
\hline
\end{tabular}

Abbreviations: Core $=$ Core disgust BATs, AR $=$ Animal-reminder disgust BATs, IP $=$ InterPersonal disgust BATs, SM $=$ SocioMoral disgust BATs; $\mathrm{ED}=$ Experienced Disgust, $\mathrm{EH}=$ Experienced Horridness, $\mathrm{EHA}=$ Experienced Happiness. Note: Standard deviations are presented in parentheses.

\section{Correlation between trait and state disgust indices during BATS}

Additionally, bivariate correlations were computed between the two DPSS-R subscales (DPSSRP, DPSS-RS) and the mean score of the state emotions (experienced disgust, horridness, and happiness) during the first step of each BAT. For the purpose of examining the convergent validity, total scores of the other disgust propensity indices (DES, DQ, DS) were also included in the analyses.

Table 7 shows that for experienced disgust, all disgust propensity indices (DPSS-RP, DES, DS, DQ) were significantly and comparably correlated with mean experienced disgust for the overall mean of the first steps of all BATs. Thus, higher disgust propensity before the start of the experiment was associated with a greater experience of disgust during the BATs. 


\section{CHAPTER 4}

All disgust propensity indices displayed similar correlations with the experienced disgust for the first step of the domain-specific BATs (Core, A-R, IP), with the exception that the DPSS-RP correlated only border-significantly with the experienced disgust for the Core BATs.

Table 7.

Bivariate correlations between disgust indices (DPSS-R, DES, DS, DQ) and the mean of the VAS-scores (Experienced Disgust, Horridness and Happiness) during the first step of each BAT (Core, AR, IP, SM, overall mean total).

\begin{tabular}{|c|c|c|c|}
\hline Questionnaires & ED & $\mathrm{EH}$ & EHA \\
\hline \multicolumn{4}{|l|}{ Core } \\
\hline DPSS-RP & .24 & .24 & -.02 \\
\hline DPSS-RS & .19 & .23 & -.17 \\
\hline DES total & $.35 * *$ & $.26 *$ & .23 \\
\hline DS total & .23 & $.38 * *$ & .09 \\
\hline DQ & $-.32 *$ & $-.27 *$ & .04 \\
\hline \multicolumn{4}{|l|}{$A-R$} \\
\hline DPSS-RP & $.36^{* *}$ & $.30 *$ & -.02 \\
\hline DPSS-RS & $.26 *$ & .17 & -.16 \\
\hline DES total & $.37 * *$ & $.33 *$ & .18 \\
\hline DS total & $.34 * *$ & $.30 *$ & .07 \\
\hline $\mathrm{DQ}$ & $-.37 * *$ & -.21 & .03 \\
\hline \multicolumn{4}{|l|}{$\underline{\mathrm{IP}}$} \\
\hline DPSS-RP & $.32 *$ & $.27 *$ & .02 \\
\hline DPSS-RS & $.27 *$ & $.27 *$ & -.16 \\
\hline DES total & $.33 *$ & .15 & $.30 *$ \\
\hline DS total & $.39 * *$ & $.38^{* *}$ & .09 \\
\hline $\mathrm{DQ}$ & $-.27 *$ & -.03 & .01 \\
\hline \multicolumn{4}{|l|}{ SM } \\
\hline DPSS-RP & .11 & .14 & -.01 \\
\hline DPSS-RS & .04 & -.01 & -.11 \\
\hline DES total & .18 & .12 & $.26 *$ \\
\hline DS total & .18 & .25 & .11 \\
\hline DQ & .04 & .11 & .01 \\
\hline \multicolumn{4}{|l|}{ Mean total } \\
\hline DPSS-RP & $.33 *$ & $.27 *$ & -.01 \\
\hline DPSS-RS & .22 & .18 & -.15 \\
\hline DES total & $.38^{* *}$ & $.26 *$ & .25 \\
\hline DS total & $.35 * *$ & $.37 * *$ & .09 \\
\hline $\mathrm{DQ}$ & $-.29 *$ & -.11 & .01 \\
\hline
\end{tabular}

Abbreviations: Core $=$ Core disgust BATs, A-R $=$ Animal-reminder disgust BATs, IP = InterPersonal disgust BATs, SM $=$ SocioMoral disgust BATs, ED $=$ Experienced Disgust, $\mathrm{EH}=$ Experienced Horridness, $\mathrm{EHA}=$ Experienced Happiness, DPSS-RP = Disgust Propensity and Sensitivity Scale-Revised Propensity, DPSS-RS $=$ Disgust Propensity and Sensitivity Scale-Revised Sensitivity, DS-total $=$ Disgust Scale, DES $=$ Disgust Emotion Scale, DQ $=$ Disgust Questionnaire. * Significant at $\mathrm{p}<.05 ; * *$ Significant at $\mathrm{p}<.01$ 
Overall, no correlations were observed between the propensity indices and the sociomoral BATs. For experienced horridness, the propensity indices DPSS-RP, DES, and DS were significantly correlated with mean experienced horridness during the first step of the BATs. The DQ only correlated significantly with horridness during the Core-BATs. In general, disgust sensitivity did not correlate significantly with the state emotions. Further, overall, none of the disgust indices correlated with the unrelated control emotion happiness.

\section{Discussion}

The major aim of the present study was to investigate the predictive value of the DPSS-R with respect to disgust-induced psychophysiological reactivity and avoidance behavior. The main findings are: i) the DPSS-R propensity and sensitivity subscales showed no predictive value for physiological reactivity during a disgust-inducing film, yet ii) higher scores on disgust propensity were associated with stronger avoidance behavior and state disgust on a series of disgust-BATs, and iii) higher scores on disgust sensitivity were associated only with performance on the most prototypical disgust-BATs (Core).

The DPSS-R is the only instrument to date that measures both disgust propensity and disgust sensitivity. Earlier research using factor analyses showed that these two factors can indeed be reliably established (Olatunji et al., 2007; van Overveld et al., 2006). To further examine the usefulness of the DPSS-R as a measure of disgust propensity, the major aim of the present study was to examine its predictive validity. More specifically, we predicted that elevated levels of disgust propensity would be associated with increased physiological reactivity during a disgust film, whereas both disgust propensity and sensitivity would be predictive of less approach behavior in a series of disgust-inducing behavioral experiments.

In the first part of the study, parasympathetic activity and facial EMG were measured while participants viewed a disgusting film. Attesting to its validity as a disgust elicitor, the film gave rise to an increase in experienced disgust as well as to disgust-specific muscular activity of the levator. In support of the view that disgust is characterized by increased parasympathetic activity (e.g., Levenson, 1992), there was an increase in HF power together with a decrease in heart rate frequency during the disgust-film. Further, there was an increase in activity of the $\mathrm{m}$. digastricus and in physiological arousal (as indicated by a decrease in T-wave amplitude and an increase in skin conductance level). Yet, neither the disgust sensitivity nor propensity subscale of the DPSS-R showed predictive validity for experienced disgust or physiological responsivity.

In apparent contrast with this, the DES did display predictive validity for the reactivity in the HF power band of heart rate variability while the DS also correlated with two disgust-relevant physiological parameters (i.e., levator activity and HF power band). In addition, both DES and DS correlated with experienced disgust, whereas the DPSS-R did not. This could be due to content-overlap between specific items on the DES / DS and the movie (all included 'vomit'). In line with this, no association was evident between scores on the DQ (a measure of core disgust without reference to 'vomit') and physiological and/or subjective disgust-relevant reactivity. This suggests that enquiring about specific items may render it more easy for participants to vividly 


\section{CHAPTER 4}

imagine their response and thus to provide more accurate ratings. Given that the DPSS-R did not correlate with physiological reactivity, the data suggest that physiological reactivity may be bound to specific items. This implicates that if researchers are interested in disgust-reactivity for specific items, the DPSS-R may not be the best suitable option. However, it may be the most suitable index when interested in general disgust propensity rather than disgust propensity for specific objects.

The second part of the study examined the predictive validity of the DPSS-R in relation to disgust-induced avoidance behavior. Supporting the validity of the DPSS-R as an index of trait disgust propensity, the present data confirmed that disgust propensity (as indexed by DPSS-RP) was associated with a decreased willingness to complete a series of disgusting behavioral experiments. This supports earlier studies that showed that higher levels of disgust propensity (as measured by the traditional indices) were associated with the individuals' performance on various behavioral experiments (Deacon \& Olatunji, 2007; Tsao \& McKay, 2004). Further, disgust propensity as indexed by the DPSS-RP and DS proved the best predictors for the total number of steps taken on the BATs. This is particularly noteworthy since the DPSS-R measures disgust propensity irrespective of specific elicitors. So, associations between disgust propensity and behavioral avoidance tendencies for specific objects can not be attributed to content overlap.

Further, in accordance with an earlier validation study of the DS (Rozin et al., 1999), there appeared congruency between the level of disgust that people claim to experience when confronted with specific objects (as indexed by DES / DS) and what they would actually do upon confrontation with them in a series of behavioral experiments. Moreover, this relationship was strengthened by the observation that levels of self-reported disgust propensity (as indexed by DES and DS) appeared associated with disgust-induced parasympathetic physiological reactivity (i.e., HF-power). In other words, what people say and what people do matched considerably. With respect to the DPSS-R, most notably is the observation that although the DPSS-R does not enquire about specific elicitors, similar associations were observed between experiencing disgust and the decision to fully complete the behavioral experiments.

The prediction that disgust sensitivity would also be associated with behavioral avoidance was not confirmed by the regression analyses. However, disgust sensitivity did correlate significantly with the number of steps taken in the core and border-significantly with the number of steps in the A-R disgust BATs. Perhaps disgust sensitivity is only relevant for prototypical disgust elicitors and not for elicitors from the more abstract domain of SM disgust. Earlier research already noted that $A-R, I P$, and SM disgust may have originally evolved from core disgust (Rozin, Haidt, \& McCauley, 1999). If so, then the further these concepts have evolved from the original construct (e.g., in case of IP or SM disgust), the less clear the association with disgust might be to the participants. It is not unlikely that even when measuring disgust without reference to specific elicitors, the strongest associations should be found for the more central forms of disgust (i.e., core and AR disgust). Accordingly, the DPSS-R subscales showed relatively strong correlations with avoidance behavior during the core and the A-R BATs, slightly less for the IP BATs, and even weaker for the SM BATs. Although the data may indicate that the pattern differs somewhat for disgust propensity and sensitivity with the 
separate BAT domains (e.g., DPSS-RP correlated significantly with IP BAT performance, while DPSS-RS did not), it is not unlikely that since correlations between propensity and BAT performance were somewhat higher in general, this may reflect a quantitative rather than a qualitative difference. Yet, a similar pattern was absent for the DS and DES that measure disgust for specific elicitors across multiple disgust domains. Moreover, none of the state or trait disgust indices was associated with behavioral withdrawal during the SM BATs. Future research should take several issues into account. First, it might be that the range of the DPSS-RS was not sufficiently extreme in the present sample to appropriately test the idea that elevated levels of disgust sensitivity influence behavioral withdrawal for disgust-inducing stimuli. The finding that the mean as well as the maximum scores (26 in a range of $8-40$ ) for disgust sensitivity were relatively low is consistent with such a view.

Second, researchers should consider their research goals closely in order to determine whether a stimulus-dependent (i.e., DES, DS) or stimulus-independent (i.e., DPSS-R) index of disgust propensity is the most appropriate. Not all participants who are disgusted more readily will be disgusted more readily of all the items that are incorporated in the DES and DS. Yet, those who do report disgust more readily for stimuli of the DES and DS, will most likely also display stronger physiological reactivity for these items. This is particularly likely since disgustinduced reactivity may depend heavily on the type of stimulus used. This may explain why the DES and DS correlated moderately with physiological reactivity while the DPSS-R did not. Additionally, it suggests that the DPSS-R indeed measures a general form of trait disgust propensity and sensitivity, while the DES and DS appear stimulus-dependent.

Third, the majority of the participants experienced relatively low disgust during the BATs. This could indicate that not all BATs were equally effective in inducing disgust. However, in general, participants did report significant increases of disgust and horridness during the BATs and the film. The low levels of state disgust during the BATs could be the result of our selection of participants since the participants' DPSS-R scores did not represent the full scoring range. In addition, deciles were used to obtain an adequate reflection of the variability of scores on disgust sensitivity. Thus, only a small minority of the participants scored in the high range of the DPSS-RS. Future work may therefore consider selecting two contrast groups based on their DPSS-R scores.

In conclusion, the present study investigated the predictive validity of the DPSS-R for disgust-induced physiological reactivity and avoidance behavior. The predictive validity of disgust propensity (as indexed by DPSS-RP) was good, while for disgust sensitivity (as indexed by the DPSS-RS), this remains to be established. Thus, the present study confirms earlier research (Rozin et al., 1999) that what people report to experience for various items (DES and DS) corresponds with actual behavioral tendencies. Since the DPSS-R only assesses bodily experiences associated with disgust, it extends these findings by showing that the experience of disgust is related to behavioral withdrawal as well. All in all, the present findings indicate that the DPSS-R provides complementary information compared to the presently available indices since it may measure a more broad and general construct of disgust propensity than the stimulus-dependent indices. 
The following pictures display various of the behavioral experiments.

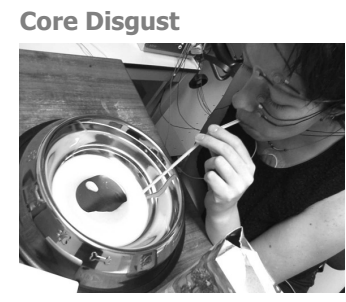

Drinking from a dog bowl

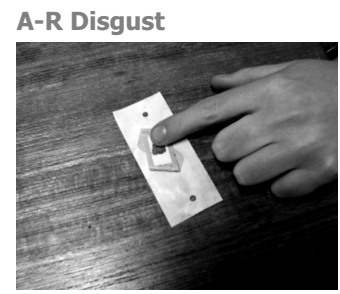

Touching bloody gauze

IP-Disgust

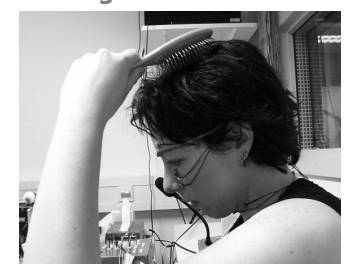

Combing hair with used brush

SM-Disgust

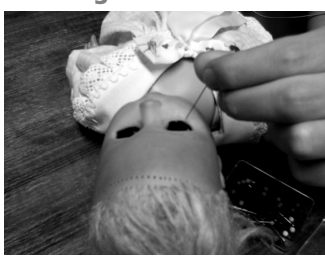

Pinning a needle through a doll's eye Agreeing to see disgusting film

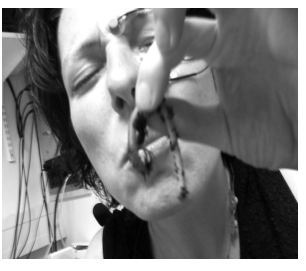

Kissing a worm

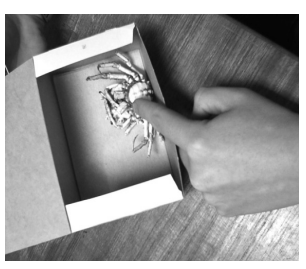

Touching a dead spider crab

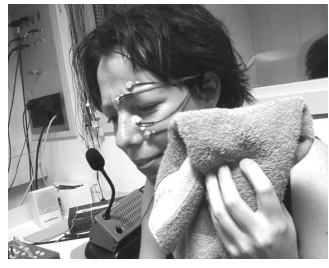

Wiping face with a used towl

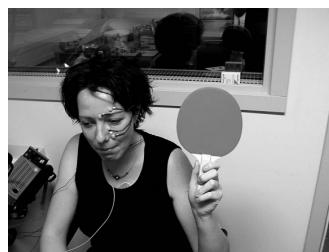

Note that these photographs were taken during a test session. 


\section{Chapter 5}

Differential startle modulation during fear and disgust: influence of individual differences in disgust propensity and sensitivity 


\section{CHAPTER 5}

\section{Abstract}

The eyeblink startle response is potentiated when probes are presented in the presence of fearful stimuli and possibly also during disgust. The strength of fear-potentiated startle has been found to vary as a function of individual differences in trait anxiety (Caseras et al., 2006). To explain inconsistent findings regarding startle potentiation during exposure to disgusting stimuli, this experiment tested whether individual differences in disgust propensity and sensitivity similarly affect disgust-induced startle modulation. Therefore, 48 women viewed a series of slides (positive, neutral, fear, disgust); during 2/3 of the slides startle probes were presented. Facial EMG ( $m$. corrugator and $\mathrm{m}$. levator labii) was assessed during unprobed slides. Levator activity increased specifically during disgust-slides. Supporting the view that disgust gives rise to startle potentiation, the magnitude of the startle response during disgust slides correlated with activity of the $\mathrm{m}$. levator labii during unprobed disgust slides. Yet, the startle reflex was not generally enhanced during fear and disgust slides. Individual differences in self-reported disgust propensity or sensitivity were not associated with startle potentiation during disgust slides. 
The eyeblink startle response is a protective response that occurs when an unexpected probe is presented (e.g., a loud tone). This defensive reflex has been found to be modulated by the individual's emotional state (Lang, Bradley, \& Cuthbert, 1990) and provides an index for measuring behavioral tendencies. Accordingly, it has repeatedly been shown that the startle response is potentiated when elicited during negative emotional states and inhibited when participants are probed in the context of positive emotional states (Filion, Dawson, \& Schell, 1998; Lang et al., 1990; Vrana, Spence, \& Lang, 1988). Thus, potentiated startles have been associated with enhanced levels of fear and anxiety (Cook, Hawk, Davis, \& Stevenson, 1991). Since disgust shares the critical features of fear as being a negative emotion that motivates avoidance behavior (e.g., Woody \& Teachman, 2000), it seems reasonable to assume that disgust will similarly give rise to startle potentiation.

However, only few studies specifically examined potentiation during disgust and the results of these studies are not very consistent. In a first study addressing this issue, it was found that startles were potentiated during fear slides (e.g., a bicyclist in flames) but not during disgust slides (e.g., mutilated faces, boy licking a cow) (Balaban \& Taussig, 1994). Yet, subsequent research provided evidence to suggest that the startle reflex nevertheless does increase during the presentation of disgusting foreground stimuli (e.g., mutilated bodies), although startle potentiation appeared most pronounced during fear-related slides (e.g., attacking animals) (Bradley, Cuthbert, \& Lang, 1999). In apparent conflict with this, a more recent study revealed an opposite pattern showing that startle potentiation was most pronounced for disgusting slides (e.g., mutilation scenes) (Yartz \& Hawk, 2002).

Recent work indicated that part of these inconsistent findings may perhaps be attributed to individual differences in personality traits such as trait anxiety (Caseras, Fullana, Riba, Barbanoj, Aluja, \& Torrubia, 2006). Accordingly, Caseras et al. (2006) found that individual variation in trait anxiety was related to startle potentiation during the presentation of fear images. However, no differences were observed between high and low trait anxious groups in startle reflex modulation during disgust slides. This shows that the interaction between individual differences in personality (i.e., trait anxiety) and image content (i.e., fear or disgust) is potentially important for understanding startle reflex modulation.

Following on from this, it may well be that startle modulation during disgusting foreground stimuli similarly varies as a function of individual differences in trait disgust. Trait disgust has recently been shown to consist of two factors, disgust propensity and disgust sensitivity (van Overveld, de Jong, Peters, Cavanagh, \& Davey, 2006). Disgust propensity reflects the tendency of the individual to respond with the emotion of disgust, whereas disgust sensitivity can be defined as the negative evaluation of the experience of disgust. Individual variation in disgust propensity and disgust sensitivity may contribute to a further understanding of the apparently inconsistent reports with respect to disgust-induced startle modulation (e.g., Balaban \& Taussig, 1994; Bradley et al, 1999; Vrana et al., 1988). Therefore, the aim of the present study was to examine if startle potentiation also occurs during disgust, and whether enhanced startle reflex modulation during presentation of disgusting images is related to individual differences in disgust propensity and sensitivity. 


\section{CHAPTER 5}

To verify the role of disgust in enhancing startle probe responses, we also measured EMG responsivity of the $\mathrm{m}$. levator labii that has been shown to be a specific marker of disgust (Vrana, 1993).

\section{Methods}

\section{Participants.}

Following classes and lectures, students from the faculty of Health Sciences at Maastricht University were randomly invited to participate in the present study $(n=48)$. As the majority of students at the faculty of Health Sciences consists of females, finding sufficient male volunteers for a reliable study of gender effects would be extremely difficult. Therefore, we preferred a homogeneous sample of only female participants. All participants were undergraduate students, with a mean age of 21.6 years $(S D=2.9)$.

\section{Questionnaires}

Disgust Propensity and Sensitivity Scale-Revised (DPSS-R; van Overveld et al., 2006). The DPSS-R is a 16-item questionnaire that contains two subscales measuring disgust propensity (the tendency to experience disgust more rapidly) and disgust sensitivity (the tendency to evaluate the disgust experience more negatively). Participants rate if they agree with a series of statements on how often they experience certain symptoms on a 5-point scale from 'never' to 'always' (range 16-80). The Dutch version of the DPSS-R is internally consistent (Cronbach's alpha ranging between .87 and .92; van Overveld et al., 2006).

Disgust Scale (DS; Haidt, McCauley, \& Rozin, 1994). The DS examines disgust propensity for a broad range of disgust elicitors. The first part contains only true/false type questions on which the person rates if they agree with a series of statements, the second part consists of 0 to 2 ratings of how disgusting they would feel during specific experiences (range 0-32). Recent research indicates that next to the total score, two subscales can be reliably established, measuring core disgust (disgust towards the prospect of oral ingestion of possible contaminants) and animal-reminder disgust (A-R) (disgust towards objects that remind humans of their animal nature) (Olatunji et al., 2007). In the literature, the A-R subscale of the DS is associated with fear of blood (e.g., de Jong \& Merckelbach, 1998), and thus with blood and blood-related stimuli.

\section{Apparatus}

EMG recordings. EMG activity of the $\mathrm{m}$. levator labii, $\mathrm{m}$. corrugator supercilii and the $\mathrm{m}$. orbicularis oculi were measured using Beckman $\mathrm{Ag} / \mathrm{AgCl}$ electrodes (diameter: $2 \mathrm{~mm}$ ). The muscle activity was measured by filling the electrodes with Spectra Electrode Gel. Before 
attaching the electrodes, the skin was cleaned with alcohol. After this, a pair of electrodes was used for each muscle at the following designated places as recommended by Fridlund \& Cacioppo (1986): at the left side of the nose ( $\mathrm{m}$. levator labii), under the left eye ( $\mathrm{m}$. orbicularis oculi) and above the left eye brow ( $\mathrm{m}$. corrugator supercilii). All EMG-signals were led through Picker Schwarzer couplings (ED 14/12) to a PXI data-acquisition system (PXI 4472; National Instruments) using a low pass filter of $1.60 \mathrm{~Hz}$ and a high pass filter of $300 \mathrm{~Hz}$ with a sample rate of $1000 \mathrm{~Hz}$ frequency. A notch filter was used to exclude $50 \mathrm{~Hz}$ interference.

\section{Materials}

Stimulus material; Participants were presented with slides depicting the following categories: positive, neutral, fear, and disgust. Recent investigations indicated that separate domains of disgust elicitors can be reliably distinguished (Olatunji et al., 2007). Consequently, in line with earlier work by Yartz and Hawk (2002), we included slides of stimuli from both categories of disgust (disgust-blood and disgust-other) in the present design.

The positive slides and the neutral slides examined whether startle augmentation would indeed only occur during negatively valenced slides (it was expected that startle magnitudes would be attenuated during positive slides with intermediate startle responding during neutral slides). The fear and disgust slides were added to test whether startle magnitudes were increased during those slide categories compared to neutral slides. The slides depicting the emotional scenes were selected from the International Affective Picture System (IAPS; Lang, Bradley \& Cuthbert, 1996) and have been used in previous studies at Maastricht University§ (e.g., Huijding \& de Jong, 2007). Each affective category consisted of twelve slides. Twelve different versions were made with regards to the sequence of presenting the slides, and each version was presented to four participants. In these versions, twelve clusters were made consisting of four slides, containing one slide of each category. Also, the following cluster always started with a slide of a different category than the last slide of the previous cluster to prevent cross-over effects. The slides were displayed using a Kodak caroussel slide projector. For the presentation of both slides and startle probes, a Compaq deskpro 486 PC was used. As acoustic probes, short $105 \mathrm{~dB}$ bursts of 50 ms 'pink noise' with an almost instantaneous rise time of 300 ns were used. While a cd containing pink noise played during the entire experiment, short signals of pink noise were delivered to the headphones by means of computer-driven gating (CMOS analog switch AD 7511). For each affective category, the probes were presented 8 times in combination with a slide and 4 times without. To prevent habituation effects, probes have also been administered 10 times without the presentation of a slide. The probes were presented at random between 3 to 5 seconds after initiation of a slide. Each slide was presented for 6 seconds. The time interval between slides varied from 10 to 20 seconds.

$\S$ The selected slides in each category had the following IAPS-numbers: positive $(146,161,171,175,192,204,205$, 207, 208, 216, 254, and 266); neutral (615, 700, 701, 703, 704, 705, 708, 709, 710, 715, 719, and 749); fear (105, $222,269,623,625,626,635,637,651,654,900$, and 981); disgust-blood (300, 301, 306, 313, 315, and 340); and disgust-other $(273,736,738,930,932$, and 957). 


\section{CHAPTER 5}

Also, if a probe was presented here, it was done in the interval of two seconds following the presentation of the previous slide to two seconds before the presentation of a new slide.

\section{Procedure}

On arrival in the laboratory, participants signed an informed consent form, after which the electrodes were attached. Next, participants viewed the slide show, during which the affective startle modulation took place. Also, physiological measurements of both EMG and skin conductance response were recorded throughout the slide show. After the slide presentation, the participants had to complete a series of questionnaires. Finally, the electrodes were removed and the participants received a small, financial reward.

\section{Data reduction and analysis}

Startle magnitudes were analyzed off-line, using a specifically designed software programme. The startle magnitude was defined as the highest level in EMG in the $150 \mathrm{~ms}$ following the initiation of a probe and was corrected for baseline-levels (mean EMG level in the $50 \mathrm{~ms}$ preprobe interval) (see also de Jong, Visser, \& Merckelbach, 1996). Approximately $2 \%$ of the trials were excluded from analyses, due to an unstable baseline EMG level. Mean magnitude for the eight startle probes per affective category (i.e., neutral, positive, fear, disgust) was calculated. The inter-slide startle responses have been excluded from the analyses, as they were only used to prevent habituation effects. Due to technical difficulties, the data of the startle magnitudes were lost for one person and for three persons for the first slides ( $0.27 \%$ total loss of data). Startle magnitudes were derived for each slide. Then, in accordance with Caseras et al. (2006), z-scores for the startle magnitudes were derived by deviating the raw scores from the individual's mean startle magnitude, and dividing this by the individual's standard deviation. From these z-scores, a mean score for each affective category (positive, neutral, fear, disgust) were obtained. The facial EMG during the un-probed slides was used to calculate mean corrugator and levator activity, and to determine whether startle reactivity during disgust slides would correspond with disgust-specific EMG activity (i.e., activity in the m. levator). Corrugator data were lost for one person. Signals were digitally rectified off-line and integrated with a time constant of 0.3 seconds via specifically designed software. To correct for the skewed normal distribution of the EMG data, a root transformation was applied. Next, mean activity for the $\mathrm{m}$. corrugator supercilli and $\mathrm{m}$. levator labii were calculated for both the 2 second period prior to each affective slide, and the 6 second period during which the slides were displayed. Mean activity during the neutral, positive, fear and disgust slides was then calculated by subtracting the 2-s prestimulus level from the activity during the slides. 


\section{Results}

\section{Descriptives}

In Table 1, the means and standard deviations are presented for the questionnaires on disgust propensity and sensitivity, as well as for disgust propensity for specific domains (i.e., core disgust and animal-reminder disgust).

Table 1.

Mean scores and standard deviations on the disgust indices for all participants.

\begin{tabular}{llll}
\hline Indices & Mean & SD & Actual Range (Potential Range) \\
\hline DPSS-RP & 22.42 & 3.09 & $16-29(8-40)$ \\
DPSS-RS & 16.27 & 3.87 & $9-27(8-40)$ \\
DS-total & 15.00 & 3.46 & $7-23.5(0-32)$ \\
DS-Core & 10.50 & 2.63 & $4-15(0-16)$ \\
DS-A-R & 8.88 & 2.56 & $3-12(0-16)$ \\
\hline Valid N & & 48
\end{tabular}

Abbreviations: Disgust Propensity and Sensitivity Scale-Revised Propensity (DPSS-RP), Disgust Propensity and Sensitivity Scale-Revised Sensitivity (DPSS-RS), Disgust Scale-Core, Disgust (DS-Core), Disgust Scale - AnimalReminder (DS-A-R).

\section{Facial EMG}

First, we examined whether specifically the disgust slides would evoke disgust-specific EMG activity (i.e., the m. levator labii). A 2 (EMG: Corrugator, Levator) x 4 (Slide Category: mean activity during neutral, positive, fear, and disgust slides) ANOVA revealed a significant interaction term EMG x Slide Category $\left(F(3,44)=6.65 ; p<.01 ; \eta_{p}^{2}=.31\right)$ suggesting that the corrugator and levator reacted differently during the various slide categories.

Next, a series of post-hoc paired sample t-tests revealed that corrugator activity was significantly higher during positive slides $(t(46)=3.90 ; p<.01 ; d=.58)$ and the disgust slides $(t(46)=-3.83 ; p<.01 ; d=.65)$ compared to neutral slides. Corrugator activity did not differ between the fear and neutral slides $(t(46)=1.37 ; p=.18 ; d=.22)$.

For the $\mathrm{m}$. levator, post-hoc paired sample t-tests showed that activity in the $\mathrm{m}$. levator was significantly higher during disgust slides compared to the neutral slides $(t(47)=-3.45 ; p<.01$; $d=.67)$, whereas it was significantly reduced during the positive slides compared to neutral slides $(t(47)=3.38 ; p<.01 ; d=.53)$. Activity in the $\mathrm{m}$. levator during fear slides did not differ from activity during neutral slides $(t(47)=.79 ; p=.44 ; d=.10)$. So, although both facial muscles were activated during disgust slides, the $\mathrm{m}$. levator was activated specifically during the disgust slides.

\section{Startle magnitudes}

Results for the affective startle blink magnitude in the different slide categories have been studied using repeated measures ANOVA analyses. First, to test whether we could replicate the 


\section{CHAPTER 5}

often reported affect-modulated startle pattern, a linear trend analysis was performed to study whether a linear trend could be observed in mean startle magnitude during the different emotionally valent slides (positive, neutral, and the mean of all negatively valent slide categories: fear and disgust). As can be seen in Figure 1, tests of the general estimated functions revealed a linear trend in the expected direction $\left(f(1,46)=4.28 ; p<.05 ; \eta_{\mathrm{p}}{ }^{2}=.09\right)$. Next, a repeated measures ANOVA was conducted for the startle reactivity during the negatively valent slide categories (Slides: mean startle during fear minus mean magnitude during neutral, mean magnitude during disgust minus mean startle magnitude during neutral). The intercept indicated that compared to the startle reactivity during the neutral category, overall, no increases in startle activity were noted during fear or disgust slides $(f(1,46)=2.32$; $\left.p=.14 ; \eta_{p}^{2}=.05\right)$. Also, the main effect of Slide was not significant $(F(2,45)=.88 ; p=.42$; $\left.\eta_{p}{ }^{2}=.04\right)$ indicating that the different negatively valent slide categories did not evoke different magnitudes of startle reactivity.

Next, a series of a priori contrasts were tested to determine whether startle reactivity (startle magnitudes during the affective slides minus the startle magnitudes during neutral slides) was significantly different from zero. For the startle magnitude during the fear slides, a border-significant increase $(t(46)=1.85 ; p=.07 ; d=.27)$ was found. For the disgust slides, reactivity did not significantly differ from the reactivity during the neutral slides $(t(46)=1.20$; $p=.24 ; d=.18)$.

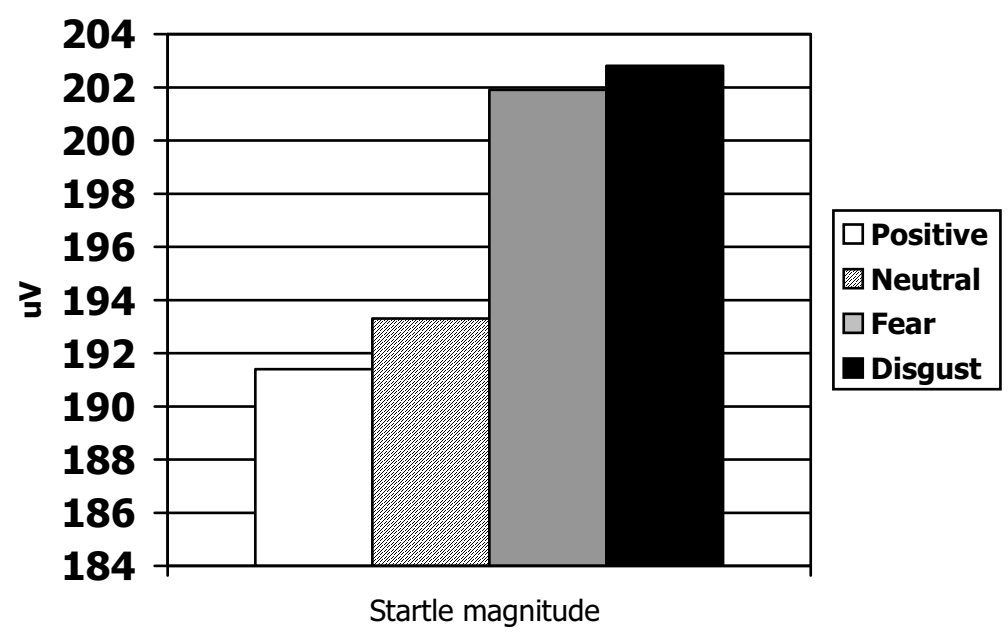

Figure 1. Startle reactivity (mean startle magnitude during affective slides) for all slides (positive, neutral, fear, disgust). 


\section{Correlations between facial EMG and startle reactivity}

Table 2 displays correlations between facial EMG reactivity (mean reactivity in facial EMG during affective slides minus activity during neutral slides) and the startle reactivity (startle magnitude during the affective slides minus the activity during neutral slides) during the presentation of the emotionally valent slides. Levator activity was correlated with startle potentiation during disgust slides but not during fear slides. Corrugator activity was not associated with startle potentiation during disgust or fear slides.

Table 2.

Bivariate Pearson correlations between facial EMG reactivity and startle reactivity during negatively valent slides.

\begin{tabular}{lll}
\hline Startle magnitude during & Fear & Disgust \\
\hline Corrugator & -.14 & .19 \\
Levator & -.02 & $.38^{* *}$ \\
\hline Valid N & & 47 \\
\hline$*=p<.05 ; * *=p<.01$ & &
\end{tabular}

Correlation between the DPSS- $R$ and reactivity in facial EMG

Bivariate Pearson correlations were calculated between the DPSS-R subscales (disgust propensity and disgust sensitivity) and reactivity in facial EMG (activity during emotionally valent slides minus activity during neutral slides). Table 3 shows that no correlations were observed between the self-report questionnaire and facial EMG reactivity during the negatively valent slides (fear and disgust).

Table 3.

Bivariate Pearson correlations between the DPSS-R subscales and facial EMG reactivity during the negatively valenced slides (activity during fear / disgust slides minus activity during neutral slides).

\begin{tabular}{lllll}
\hline & Fear & \multicolumn{2}{l}{ Disgust } \\
\cline { 2 - 5 } & Corrugator & Levator & Corrugator & Levator \\
\hline Propensity (DPSS-RP) & .21 & -.11 & .18 & -.10 \\
Sensitivity (DPSS-RS) & .09 & .04 & .05 & .02 \\
\hline Valid N & & & 48
\end{tabular}

\section{Contribution of disgust propensity and sensitivity to startle reactivity}

Regression analyses examined whether disgust propensity and sensitivity contributed independently to the variance in startle reactivity during disgust slides. Startle reactivity (startle magnitude during disgust minus startle magnitudes during neutral slides) was taken as dependent variable and scores on DPSS-R subscales were included as predictors. Startle reactivity during disgust was not predicted significantly by disgust propensity $(\beta=.00 ; t=.02$; $p=.98)$ or sensitivity $(\beta=.02 ; t=.80 ; p=.43)$.

Similarly, regression analyses were conducted using startle reactivity (startle magnitude during disgust minus startle magnitudes during neutral slides) as dependent variable and both 


\section{CHAPTER 5}

DS-Core and DS-A-R as predictor variables. Startle reactivity during disgust was not predicted significantly by propensity for core disgust $(\beta=-.02 ; t=-.60 ; p=.55)$ or animal-reminder disgust ( $\beta=.04 ; t=1.06 ; p=.29$ ). Thus, neither individual differences in self-reported disgust propensity and disgust sensitivity nor self-reported disgust propensity for separate domains of disgust, influenced the modulation of the affective eye blink startle reflex.

Lastly, regression analyses with startle reactivity (startle magnitude during fear minus startle magnitudes during neutral slides) as dependent variable and DPSS-R subscales as predictor variables showed that startle reactivity during fear slides was not significantly predicted by disgust propensity $(\beta=.02 ; t=.64 ; p=.53)$ or sensitivity $(\beta=.01 ; t=.06 ; p=.95)$. Similarly, regression analyses investigated whether core and animal-reminder disgust propensity contributed to startle reactivity (startle magnitude during fear slides minus startle magnitudes during neutral slides). Startle reactivity was used as dependent variable and the DS-Core and DS-A-R scales were included as predictors. Startle reactivity during fear was not significantly predicted by propensity for core $(\beta=-.03 ; t=-1.10 ; p=.28)$ or A-R disgust $(\beta=.02 ; t=.73$; $p=.47)$. Thus, individual differences in disgust propensity for specific domains did not influence startle modulation.

\section{Discussion}

The main findings are: i) eye blink startle probe reflexes were not generally enhanced during disgust and fear slides compared to the neutral condition, ii) individual differences in selfreported disgust propensity and disgust sensitivity were not related to differences in startle potentiation during disgust (or fear) evoking slides, iii) specifically during disgust slides, startle potentiation was significantly correlated with activity of the disgust-specific $\mathrm{m}$. levator labii.

The goal of the present study was to examine the role of individual differences in disgust propensity and sensitivity in the modulation of the eye blink startle reflex. In line with earlier findings and attesting to the validity of the present approach (Hamm, Greenwald, Bradley, \& Lang, 1993; Cook et al., 1991), startle magnitude was lowest during positive slides and highest during negatively valenced slides. Yet, although a borderline-significant increase was observed in startle potentiation during fear slides compared to neutral slides, no differences in startle potentiation was observed during disgust slides compared to neutral slides. So, in contrast to earlier reports (Yartz \& Hawk, 2002), no evidence was found that the startle reflex is generally enhanced during the presentation of disgust-relevant stimuli. Interestingly, however, the strength of startle potentiation during disgust-slides was found to be correlated with the responsivity of the (disgust-specific) $\mathrm{m}$. levator labii. This finding supports the notion that disgust may give rise to startle potentiation, but that this modulatory effect is only evident in individuals who respond with relatively strong spontaneous facial expressions of disgust. Such relatively strong facial responsivity can be taken to reflect individual differences in the extent to which the presently used stimuli were able to elicit disgust in the present sample of participants. This may be due to more general individual differences in disgust propensity and/or sensitivity. 
In the current study, no correlation was found between startle modulation during disgusting foreground slides and individual differences in either disgust propensity (as indexed by DPSS-RP and DS) or disgust sensitivity (indexed by DPSS-RS). This may be the result of individual differences in response stereotype. It is not unlikely that given a similar subjective experience on self-report, individual differences may still be observed in spontaneous, physiological responses. Thus, individual differences in physiological response stereotype could have influenced startle potentiation in the sense that the stronger participants' physiological expression of disgust, the larger the disgust-induced startle potentiation. In accordance with this, although in general startle potentiation did not occur during the disgust slides, startle reactivity during the disgust slides did correlate with the reactivity of the $\mathrm{m}$. levator labii during the unprobed disgust slides.

Since no valence ratings for the slides were obtained, the absence of a correlation between self-report and physiological indices could reflect either that individual differences in physiological response stereotype are more important to disgust-induced startle potentiation than experienced disgust, or merely that objective and subjective indices represent dissociated phenomena. Relatedly, previous research on stress showed that subjectively experienced stress and objective stress-induced responsivity of the immune response system are generally both observed, but also do not correlate well (Segerstrom \& Miller, 2004).

Although the concept of disgust sensitivity is by nature subjective, disgust propensity can be assessed relatively more objectively. Disgust propensity can be reliably established at an explicit level using self-report measures such as the DPSS-R. However, it may also be measured at a more implicit level by measuring either physiological responsivity (e.g., activity of disgustspecific facial EMG of the levator labii; de Jong, Peters, \& Vanderhallen, 2002) or implicit memory associations (e.g., using the Extrinsic Affective Simon Task; Huijding \& de Jong, 2007). The strength of physiological responsivity or of the implicit affective memory associations towards a variety of disgust-stimuli could provide an alternative and implicit index for disgust propensity. Interestingly, earlier research already demonstrated that such implicit selfassociations of trait anxiety proved to have superior predictive validity for automatic fearresponses (e.g., Egloff \& Schmulke, 2002). In a similar vein, implicit associations of disgust propensity may hold superior qualities in predicting automatic disgust behavior (cf., Gawronski, Lebel, \& Peters, 2007; Huijding \& de Jong, in press), such as disgust-induced startle potentiation. Consistent with this, recent work in our lab showed that implicit disgust-spider associations showed superior predictive value for levator activity during the presentation of a spider video clip (Hiemstra, 2007). Therefore, the present lack of a convincing relation between trait disgust and disgust-induced startle potentiation might be (partly) attributed to the fact that the DPSS-R measures disgust propensity at an explicit level. It is not unlikely that automatically activated memory associations (rather than explicit, deliberate self-reported associations) may be most important for eliciting reflective, uncontrollable fear responses such as the startle reflex (e.g., Huijding \& de Jong, 2006). To arrive at more solid conclusions in this respect, it would be important for future research to complement self-report measures with more implicit measurements of disgust propensity. 


\section{CHAPTER 5}

The present study has several limitations. First, because no generally enhanced startle reactivity was evident during the presentation of the disgust and fear slides one may question whether the present slides were intense enough to evoke meaningful levels of disgust and fear. However, this study included mostly slides that proved effective in prior research (e.g., Yartz \& Hawk, 2002). Moreover, the disgust-specific responses in facial EMG together with the association between levator activity and startle potentiation seem to attest to the validity of the present stimulus material. Furthermore, it might be that the previous study by Yartz and Hawk (2002) overestimated the strength of disgust induced startle potentiation as the category of disgusting objects was over-represented in that study. Disgusting slides were viewed twice as often as other emotional categories. This may have induced this emotion more strongly, sensitizing individuals for disgusting stimuli and leading to greater physiological responses in that earlier study. Additionally, Yartz and Hawk (2002) used the same picture presentation order for all participants. Thus, it can not be ruled out that the sequence of the slides may have confounded differential startle potentiation during the experiment. For example, if disgust stimuli are over-represented in the first half of the experiment, responses toward those stimuli may be artificially enhanced.

Second, it may be argued that the range in disgust propensity and sensitivity levels in our research population could have influenced our findings. Although the present sample included participants scoring over a broad range of the DPSS-R, the research population did not include the full range of the DPSS-R. Therefore, future studies should consider using groups with even more extreme levels of disgust propensity and sensitivity, to provide a sharper contrast between individuals.

Third, recently, state disgust was found to predict behavioral avoidance for disgusting stimuli (i.e., spiders; Woody \& Tolin, 2002). In the present study, state disgust as indexed by disgust specific facial EMG correlated with an index of behavioral avoidance (i.e., startle potentiation during disgust foreground slides). This is in line with previous research that state rather than trait anxiety influences affective startle modulation (Grillon, Ameli, Foot, \& Davis, 1993). In a similar vein, it may be that individual differences in state disgust are more important than differences in disgust propensity and sensitivity. Thus, more studies are needed to determine the relative contribution of both trait and state variables on certain behavioral avoidance tendencies. Relatedly, valence ratings should be obtained to be able to establish whether those who rate certain slides more disgusting or fear-evoking, are those who respond with greater physiological (startle) reactivity.

Fourth, previous research indicated that individual differences in trait anxiety influenced affective modulation of the startle reflex (Corr, Kumari, Wilson, Checkley, \& Gray, 1997). Based on the present findings, it can not be ruled out that startle potentiation would indeed be more closely linked with general negative affectivity (e.g., neuroticism) rather than with differential emotional states. Therefore, it would be recommendable to include an index of neuroticism in future research. Moreover, using indices of both trait disgust and trait anxiety would allow investigating their relative contribution to startle potentiation in response to disgust and fear evoking stimuli. 
In sum, the present findings indicate that self-reported individual differences in disgust propensity and disgust sensitivity do not influence startle potentiation in the presence of disgusting foreground stimuli. However, startle potentiation during disgust slides did correlate with activity in disgust specific facial EMG suggesting that state disgust influenced startle potentiation, in the sense that the stronger participants' expression of disgust the larger the increase in startle potentiation. So individual differences in physiological responsivity to disgust stimuli (in terms of levator activity) may be linked to individual differences in disgust induced startle potentiation. 
Chapter 6

Disgust propensity and disgust sensitivity are differentially associated with psychopathological symptoms 


\title{
CHAPTER 6
}

\begin{abstract}
Current indices on disgust propensity (i.e., how easily an individual experiences disgust) may inflate relationships with psychopathology indices due to conceptual overlap. Therefore, the Disgust Propensity and Sensitivity Scale-Revised (DPSS-R; van Overveld et al., 2006) was recently introduced, which measures disgust propensity irrespective of specific referents. Moreover, it is the only instrument that differentiates between disgust propensity and disgust sensitivity (i.e., how horrid does the individual consider being disgusted). To test the specificity of the relationship between disgust propensity/sensitivity and psychopathological symptoms, the present study administered the DPSS-R, together with indices of supposedly disgust-related psychopathology (e.g., fear of contamination), of a supposedly disgust-unrelated psychopathological disorder (depression), and neuroticism in a student sample $(N=216)$. Independent of neuroticism, the disgust traits displayed differential associations with psychopathological complaints. Disgust propensity was most strongly associated with fear of spiders, disgust sensitivity was most strongly associated with obsessive compulsive complaints and eating styles, while both were associated with fear of blood. This is consistent with the notion that disgust propensity and sensitivity may differentially contribute to particular psychopathological complaints.
\end{abstract}


High levels of trait disgust may set people at risk for developing certain psychopathological symptoms involving disgust or contamination-related preoccupations. Accordingly, elevated levels of disgust propensity (i.e., tendency to respond with the emotion of disgust) are observed in people with spider phobia (Mulkens, de Jong, \& Merckelbach, 1996), blood phobia (de Jong \& Merckelbach, 1998), obsessive compulsive disorder (Olatunji, Lohr, Sawchuk, \& Tolin, 2007), eating disorders (Davey, Buckland, Tantow, \& Dallos, 1998), and sexual dysfunctions (de Jong, van Overveld, Weimar-Schultz, Peters, \& Buwalda, in press).

Disgust propensity** is generally assessed using questionnaires such as the Disgust Scale (DS; Haidt, McCauley, \& Rozin, 1994) and the Disgust Questionnaire (DQ, Rozin, Fallon, \& Mandell, 1984). Unfortunately, correlations between disgust propensity and psychopathology may be inflated due to conceptual overlap between the respective assessment instruments (van Overveld, de Jong, Peters, Cavanagh, \& Davey, 2006). For example, the DS and the Vancouver Obsessional Compulsive Inventory (Thordarson, Radomsky, Rachman, Shafran, Sawchuk, \& Hakstian, 2004) both contain items on 'public toilets'. Therefore, the first aim of this study is to establish whether the association between disgust propensity and psychopathology symptoms is substantiated when a disgust index without conceptual overlap is used.

Furthermore, not only disgust propensity but also disgust sensitivity (i.e. the tendency to evaluate the experience of disgust highly negatively; van Overveld et al., 2006) may be important in the etiology and/or maintenance of psychopathological complaints. Therefore, the present study used the Disgust Propensity and Sensitivity Scale - Revised (DPSS-R; van Overveld, de Jong, Peters, Cavanagh, \& Davey, 2006), the only instrument that measures both disgust propensity and disgust sensitivity. Moreover, we propose that disgust propensity and sensitivity may be differentially related to various psychopathological disorders. High levels of disgust propensity increase the likelihood to find specific stimuli disgusting. So the often reported relationship between disgust propensity and specific fears (e.g., spiders) may be explained by assuming that spiders are more likely to acquire a disgust-evoking status in people with high levels of disgust propensity. Disgust sensitivity may motivate individuals to avoid situations at the prospect of experiencing disgust. So, for disorders in which experiencing disgust appears critically involved (e.g., blood phobia; Page, 1994; obsessive compulsive disorders; Olatunji et al., 2007), enhanced disgust sensitivity may (also) contribute to phobic complaints. Previous research already showed that disgust sensitivity was associated with blood phobia (van Overveld et al., 2006) and emetophobia (van Overveld, de Jong, Peters, van Hout, \& Bouman, 2008).

Thus, disgust sensitivity may be particularly involved in conditions involving intrinsically and universally disgusting stimuli (e.g., vomit), whereas disgust propensity could be relatively more important in disorders that are related to stimuli that are not intrinsically disgusting (such as spiders). Additionally, disgust sensitivity may be associated with contamination-related preoccupations in obsessive compulsive disorders and blood phobia. Moreover, experiencing

\footnotetext{
** Earlier research used the term 'disgust sensitivity' to describe the tendency to experience disgust more readily. In line with research on anxiety sensitivity, the present authors argued recently that the original "disgust sensitivity" questionnaires actually measure disgust propensity (see van Overveld et al., 2006). Disgust sensitivity is defined here as the tendency to evaluate the experience of disgust as an extremely unpleasant sensation.
} 


\section{CHAPTER 6}

disgust may be associated with food aversion, negative body image, and self-loathing in eating disorders.

Davey (2003) has argued that most questionnaire studies in disgust research tend to neglect two important issues. First, an index of negative affectivity is often not included, so it remains unclear whether elevated levels of disgust propensity are not merely the epiphenomenon of generally heightened negative affect. Second, most studies do not include questionnaires on psychopathological complaints that are supposedly unrelated to disgust. Thus, it is unclear whether disgust propensity and sensitivity are related to a specific class of disorders or that they are generally enhanced in all psychopathological conditions. Studies that did include indices of unrelated complaints found that these were correlated with disgust propensity (e.g., separation anxiety: Muris, Merckelbach, Schmidt, \& Tierney, 1999; acrophobia and claustrophobia: Davey \& Bond, 2006). Therefore, we included a scale on depression, which seems unrelated to disgust to test the specificity of the relationship between disgust sensitivity/propensity and psychopathology.

Thus, the present study examined the specificity of the associations between disgust propensity and sensitivity with various disorders. Disgust propensity was presumed to be associated most strongly with fear of spiders, whereas disgust sensitivity was presumed to be more important in disorders where the experience of disgust seems predominantly involved (i.e., fear of blood, obsessive compulsive disorders, and eating disorders). Both factors were expected to be unrelated to depression. Lastly, a scale on neuroticism was used to ensure that associations between trait disgust and psychopathology were independent of trait negative affectivity.

\section{Methods}

\section{Participants}

Students $(N=216)$ at the faculties of Health Sciences, Psychology, and Medicine from Maastricht University were recruited via posters and advertisements in the university paper. Since the student population at these faculties consists predominantly of women, most participants in the present study were female (78\%; 169 women, 47 men) with a mean age of 21.2 years $(S D=2.5$, range: $18-39$ years $)$.

\section{Materials}

Disgust Propensity and Sensitivity Scale-Revised (DPSS-R; van Overveld et al., 2006). This scale assesses disgust propensity and disgust sensitivity. Participants rate whether they agree to 16 propositions on how often they experience various bodily symptoms on a scale from 1 ('never') to 5 ('always'; range: 16-90). Two subscales on disgust propensity and disgust sensitivity can be calculated that are internally consistent ( $a=.75$; for both subscales in the present study; all a's > .71; van Overveld et al., 2006). 
Fear of Spiders Questionnaire (FSQ; Szymanski \& Donohue, 1995); The FSQ is an 18-item scale on fear of spiders. Ratings are given on a scale from 0 ('strongly disagree') to 7 ('strongly agree'; range: 0-126). Internal consistency is high ( $a=.97$; present study; .92; Szymanski \& Donohue, 1995).

Blood-Injury Phobia Questionnaire (BIQ; de Jong \& Merckelbach, 1998); The BIQ examines whether people are afraid of blood-injury stimuli. On the first ten items, participants rate their fear of 10 blood-injury items on a scale from 0 ('no fear') to 4 ('maximal fear'; range $=0-40$ ). Next, participants rate their fainting history for these stimuli on a scale from 0 ('never') to 2 ('often'; range: 0-20). Both subscales are internally consistent ( $a=.86$ for BIQ-Fear, $a=.63$ for BIQ-Fainting in the present study; both a's > .73; Merckelbach, Muris, de Jong, \& de Jongh, 1999).

Vancouver Obsessional Compulsive Inventory (VOCI; Thordarson et al., 2004); A subscale of this questionnaire was used on contamination fear. It consists of 11 items scored on a scale from 0 ('Not at all') to 4 ('Very much so'). Internal consistency is high ( $a=.86$ in present study; .87 - .92; Thordarson et al., 2004).

Dutch Eating Behaviour Questionnaire (DEBQ; van Strien, Frijters, Bergers, \& Defares, 1986). This questionnaire examines three eating styles: Emotional Eating (DEBQ-Emo), External Eating (DEBQ-Ext), and Restrained Eating (DEBQ-R). The DEBQ can successfully identify patients with eating disorders. Participants rate whether various eating behaviors apply to them on a scale from 1 ('never') to 5 ('always'). Internal consistency is high (all a's $>.73$ in present study; all a 's > .79; van Strien et al., 1986).

Zung Self-rating Depression Scale (ZSDR; Zung, 1965). This 20-item scale examines an individual's level of depression. Respondents indicate whether a series of propositions on the presence of depressive symptomatology applies to them on a scale from 1 ('Rarely to never') to 4 ('Often to always'). The ZSDR appears internally consistent ( $a=.82$; present study; .84; Dugan, McDonald, Passik, Rosenfeld, Theobald, \& Edgerton, 1998).

Eysenck Personality Questionnaire (EPQ-RSS-N; Eysenck \& Eysenck, 1975). This subscale of the EPQ consists of 12 items on neuroticism. Participants rate on a dichotomous format ('no'/'yes') whether a series of statements describes them well. Internal consistency is good

\section{Procedure}

Participants were invited to the department and completed a package of questionnaires in 


\section{CHAPTER 6}

\section{Results}

\section{Data reduction.}

The data were analyzed using the Statistical Package for Social Sciences (SPSS 11.0). Due to experimenter error, the DEBQ was not administered to seven participants. For all other variables, less than $10 \%$ missing values were observed on single questionnaires. Regression analyses were used to estimate missing values following recommendations by Schafer \& Graham (2002).

\section{Descriptive statistics.}

Table 1 presents the descriptives for each questionnaire. Overall, women scored higher on most indices. Nevertheless, a series of independent sample t-tests revealed that women only scored significantly higher on FSQ, $t(214)=-3.33, p<.001$, DEBQ-Emo, $t(207)=-3.99, p<.001$, and DEBQ-R, $t(207)=-4.28 ; p<.001$.

Table 1.

Means, standard deviations, ranges, and scales of all questionnaires.

\begin{tabular}{lcllcc}
\hline Questionnaires & Men $(N=47)$ & Women $(N=169)$ & Total group $(N=216)$ & range & scale \\
\hline DPSS-RP & $18.66(3.82)$ & $19.10(4.34)$ & $19.00(4.23)$ & $8-29$ & $8-40$ \\
DPSS-RS & $12.94(3.48)$ & $13.65(4.15)$ & $13.49(4.01)$ & $8-30$ & $8-40$ \\
FSQ & $7.21(17.42)$ & $21.12(27.14)$ & $18.09(25.95)$ & $0-119$ & $0-126$ \\
BIQ-Fear & $7.04(5.71)$ & $8.17(6.25)$ & $7.92(6.14)$ & $0-31$ & $0-40$ \\
BIQ-Faint & $.43(.83)$ & $.41(1.04)$ & $.42(1.00)$ & $0-7$ & $0-20$ \\
VOCI & $3.11(4.34)$ & $3.27(4.56)$ & $3.23(4.51)$ & $0-32$ & $0-44$ \\
DEBQ-Emo & $29.70(7.13)$ & $34.89(8.88)$ & $33.84(8.79)$ & $13-65$ & $13-65$ \\
DEBQ-Ext & $30.63(4.19)$ & $30.98(4.60)$ & $30.90(4.50)$ & $18-41$ & $10-50$ \\
DEBQ-R & $21.26(9.49)$ & $27.64(8.75)$ & $26.24(9.28)$ & $10-50$ & $10-50$ \\
ZSDR & $33.66(6.03)$ & $34.35(6.98)$ & $34.19(6.78)$ & $22-56$ & $20-80$ \\
EPQ-RSS-N & $3.66(2.43)$ & $4.28(2.99)$ & $4.15(2.89)$ & $0-12$ & $0-12$ \\
\hline Valid $N$ & 47 & 169 & 216 & &
\end{tabular}

Abbreviations: DPSS-RP = Disgust Propensity and Sensitivity Scale-Revised Propensity; FSQ = Fear of Spider Questionnaire; BIQ = Blood-Injury Phobia Questionnaire; VOCI = Vancouver Obsessive Compulsive Inventory; DEBQ-Emo = Dutch Eating Behaviour Questionnaire-Emotional Eating; DEBQ-Ext = Dutch Eating Behaviour Questionnaire-External Eating; DEBQ-R = Dutch Eating Behaviour Questionnaire-Restrained Eating; ZSDR = Zung Self-rating Depression; EPQ-RSS-N = Eysenck Personality Questionnaire-Neuroticism Scale.

\section{Bivariate correlations.}

Table 2 displays the Pearson correlations between disgust propensity/sensitivity and neuroticism with all psychopathology indices. Disgust propensity correlated moderately with blood phobia (BIQ-Fear) and obsessive compulsive disorders (VOCI) and low with fear of spiders (FSQ), External Eating (DEBQ-Ext). Disgust sensitivity correlated high with blood phobia (BIQ-Fear), 
moderately with obsessive compulsive disorders (VOCI), and low with External Eating (DEBQExt) and Emotional Eating (DEBQ-Emo). Unexpectedly, both disgust traits correlated low to moderately with a supposedly unrelated index of depression (ZSDR).

Table 2.

Correlations between disgust indices (DPSS-RP, DPSS-RS), neuroticism (EPQ-RSS-N), and psychopathology (FSQ, BIQ, VOCI, DEBQ, ZSDR).

\begin{tabular}{llll}
\hline Indices & DPSS-RP & DPSS-RS & EPQ-RSS-N \\
\hline FSQ & $.17^{*}$ & .11 & .12 \\
BIQ-Fear & $.29 * *$ & $.32^{* *}$ & $.15^{*}$ \\
BIQ-Faint & .02 & .11 & -.01 \\
VOCI & $.31^{* *}$ & $.49 * *$ & $.18^{* *}$ \\
DEBQ-Emo & .10 & $.21^{* *}$ & $.26^{* *}$ \\
DEBQ-Ext & $.20^{* *}$ & $.26^{* *}$ & .11 \\
DEBQ-R & .01 & .10 & .08 \\
ZSDR & $.22^{* *}$ & $.36^{* *}$ & $.65^{* *}$ \\
EPQ-RSS-N & $.23^{* *}$ & $.31^{* *}$ & \\
\hline Valid $N$ & & 216 &
\end{tabular}

Abbreviations: DPSS-RP = Disgust Propensity and Sensitivity Scale-Revised Propensity; FSQ = Fear of Spiders Questionnaire; BIQ = Blood-Injury Phobia Questionnaire; VOCI = Vancouver Obsessive Compulsive Inventory; DEBQ-Emo = Dutch Eating Behaviour Questionnaire - Emotional Eating; DEBQ-Ext = Dutch Eating Behaviour Questionnaire - External Eating; DEBQ-R = Dutch Eating Behaviour Questionnaire - Restrained Eating; ZSDR = Zung Self-rating Depression; EPQ-RSS-N = Eysenck Personality Questionnaire - Neuroticism Scale.

* Significant at $p<.05 ; * *$ Significant at $p<.01$

\section{Regression analyses.}

To examine how disgust propensity and sensitivity respectively contribute to psychopathology indices, hierarchical regression analyses were performed. The scores on disgust propensity (DPSS-RP) and sensitivity (DPSS-RS) were used as predictors, and indices of psychopathology (FSQ, BIQ-Fear, VOCI, DEBQ, ZSDR) were included as dependent variables. To control for possible gender-related differences, gender was entered in the first step. To determine whether disgust propensity and sensitivity control for neuroticism, the EPQ-RSS-N was entered in the second step. Next, to determine whether the DPSS-R scales explain additional variance, disgust propensity (DPSS-RP) was added in the third step and disgust sensitivity (DPSS-RS) in the fourth step. In this paragraph, only the final models are described. A summary of the main findings is reported in Table 3.

The gender of the participants significantly predicted scores on the FSQ, DEBQ-Emo and DEBQ-R (all $p$ 's $<.01$ ) so women displayed higher scores on fear of spiders and eating disorders compared to men. After controlling for gender, neuroticism, as well as for the other disgust trait, disgust propensity significantly predicted only blood phobia $(p=.01)$, and was a border-significant predictor for fear of spiders $(p=.06)$ and for obsessive compulsive disorders ( $p=.08)$. Disgust sensitivity significantly predicted fear of blood ( $p=.01$ ), obsessive compulsive disorders $(p<.001)$, emotional eating $(p=.04)$, external eating $(p=.01)$, and depression $(p<.01)$. Thus, disgust sensitivity was related to all disgust-related 


\section{CHAPTER 6}

psychopathology indices (except spider fear) but also with a non-disgust-related disorder. Moreover, the disgust traits demonstrated additional value to each other as after controlling for disgust propensity, sensitivity still significantly predicted various complaints.

Table 3.

Hierarchical regression analyses for gender (male / female), disgust propensity (DPSS-R-P), sensitivity (DPSS-R-S), and neuroticism (EPQ-RSS-N) as predictors of psychopathology (FSQ, BIQ, VOCI, DEBQ, ZSDR).

\begin{tabular}{|c|c|c|c|c|c|}
\hline Index & & $B$ & SE B & B & $p$ \\
\hline Analysis 1 & Step $1 R^{2}=.05, F=11.0$ & .01 & & & \\
\hline \multirow[t]{13}{*}{ FSQ } & Gender & 13.91 & 4.18 & .22 & .001 \\
\hline & \multicolumn{5}{|c|}{ Step $2 \Delta R^{2}=.01, F_{\text {change }}=2.28, p=.13$} \\
\hline & Gender & 13.34 & 4.18 & .21 & .002 \\
\hline & EPQ-N & .91 & .60 & .10 & .13 \\
\hline & \multicolumn{5}{|c|}{ Step $3 \Delta R^{2}=.02, F_{\text {change }}=4.70, p=.03$} \\
\hline & Gender & 13.13 & 4.15 & .21 & .002 \\
\hline & EPQ-N & .60 & .61 & .07 & .32 \\
\hline & DPSS-RP & .90 & .42 & .15 & .03 \\
\hline & \multicolumn{5}{|c|}{ Step $4 \Delta R^{2}<.01, F_{\text {change }}=.05, p=.83$} \\
\hline & Gender & 13.09 & 4.17 & .21 & .002 \\
\hline & EPQ-N & .57 & .63 & .06 & .37 \\
\hline & DPSS-RP & .87 & .45 & .14 & .06 \\
\hline & DPSS-RS & .10 & .49 & .02 & .83 \\
\hline Analysis 2 & \multicolumn{5}{|c|}{ Step $1 R^{2}=.01, F=1.23, p=.27$} \\
\hline \multirow[t]{13}{*}{ BIQ-Fear } & Gender & 1.12 & 1.01 & .08 & .27 \\
\hline & \multicolumn{5}{|c|}{ Step $2 \Delta R^{2}=.02, F_{\text {change }}=4.59, p=.03$} \\
\hline & Gender & .93 & 1.01 & .06 & .36 \\
\hline & EPQ-N & .31 & .14 & .15 & .03 \\
\hline & \multicolumn{5}{|c|}{ Step $3 \Delta R^{2}=.08, F_{\text {change }}=15.78, p<.001$} \\
\hline & Gender & .84 & .98 & .06 & .39 \\
\hline & EPQ-N & .18 & .14 & .08 & .22 \\
\hline & DPSS-RP & .39 & .10 & .27 & $<.001$ \\
\hline & \multicolumn{5}{|c|}{ Step $4 \Delta R^{2}=.12, F_{\text {change }}=10.39, p<.01$} \\
\hline & Gender & .71 & .96 & .05 & .46 \\
\hline & EPQ-N & .07 & .15 & .05 & .65 \\
\hline & DPSS-RP & .26 & .10 & .18 & .01 \\
\hline & DPSS-RS & .36 & .11 & .24 & .01 \\
\hline Analysis 3 & \multicolumn{5}{|c|}{ Step $1 R^{2}=.00, F=.05, p=0.83$} \\
\hline \multirow[t]{13}{*}{ VOCI } & Gender & .16 & .75 & .02 & .83 \\
\hline & \multicolumn{5}{|c|}{ Step $2 \Delta R^{2}=.03, F_{\text {change }}=7.21, p=.01$} \\
\hline & Gender & -.02 & .74 & -.02 & .98 \\
\hline & EPQ-N & .28 & .11 & .18 & .01 \\
\hline & \multicolumn{5}{|c|}{ Step $3 \Delta R^{2}=.07, F_{\text {change }}=17.27, p<.001$} \\
\hline & Gender & -.09 & .71 & -.01 & .91 \\
\hline & EPQ-N & .18 & .11 & .12 & .08 \\
\hline & DPSS-RP & .30 & .07 & .28 & $<.001$ \\
\hline & \multicolumn{5}{|c|}{ Step $4 \Delta R^{2}=.15, F_{\text {change }}=41.13, p<.001$} \\
\hline & Gender & -.26 & .65 & -.02 & .69 \\
\hline & EPQ-N & .03 & .10 & .02 & .76 \\
\hline & DPSS-RP & .13 & .07 & .12 & .08 \\
\hline & DPSS-RS & .49 & .08 & .44 & $<.001$ \\
\hline
\end{tabular}


DISGUST IN VARIOUS CLINICAL CONDITIONS

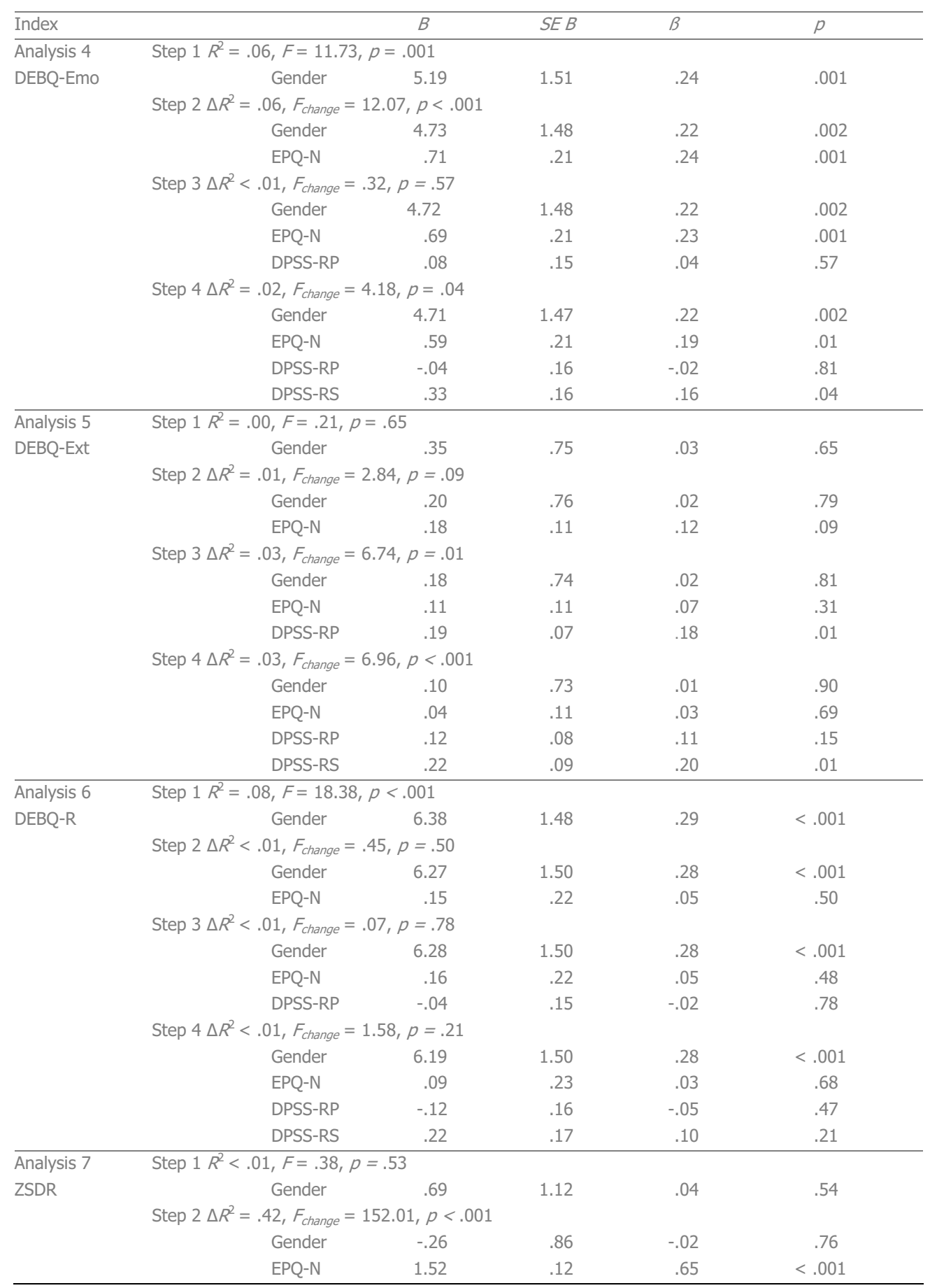




\begin{tabular}{|c|c|c|c|c|c|}
\hline Index & & $B$ & SE B & $B$ & $p$ \\
\hline \multicolumn{6}{|c|}{ Step $3 \Delta R^{2}<.01, F_{\text {change }}=1.63, p=.20$} \\
\hline & Gender & -.29 & .86 & -.02 & .74 \\
\hline & EPQ-N & 1.48 & .13 & .63 & $<.001$ \\
\hline & DPSS-RP & .11 & .09 & .07 & .20 \\
\hline \multicolumn{6}{|c|}{ Step $4 \Delta R^{2}=.02, F_{\text {change }}=8.82, p<.01$} \\
\hline & Gender & -.39 & .85 & -.02 & .65 \\
\hline & EPQ-N & 1.39 & .13 & .59 & $<.001$ \\
\hline & DPSS-RP & .01 & .09 & .01 & .94 \\
\hline & DPSS-RS & .29 & .10 & .17 & $<.01$ \\
\hline
\end{tabular}

Abbreviations: DPSS-RP = Disgust Propensity and Sensitivity Scale-Revised Propensity; FSQ = Fear of Spiders Questionnaire; BIQ = Blood-Injury Phobia Questionnaire; VOCI = Vancouver Obsessive Compulsive Inventory; DEBQ-Emo = Dutch Eating Behaviour Questionnaire - Emotional Eating; DEBQ-Ext = Dutch Eating Behaviour Questionnaire - External Eating; DEBQ-R = Dutch Eating Behaviour Questionnaire - Restrained Eating; ZSDR = Zung Self-rating Depression; EPQ-RSS-N = Eysenck Personality Questionnaire - Neuroticism Scale.

\section{Discussion}

The main findings are: i) Disgust propensity and disgust sensitivity appeared differentially related to various conditions in which disgust is assumed to be involved, ii) Associations between disgust propensity/sensitivity and psychopathology indices were independent of neuroticism, iii) Unexpectedly, both disgust traits were associated with seemingly disgustunrelated symptoms of depression.

The major goal was to test whether previously reported associations between disgustpreoccupations and psychopathology still hold when an index is used that examines this relationship irrespective of specific referents, namely, the Disgust Propensity Sensitivity ScaleRevised (DPSS-R, van Overveld et al., 2006). The finding that disgust propensity (as indexed by DPSS-RP) correlated with various psychopathology indices confirms that correlations between disgust propensity and psychopathology are not merely the result of conceptual overlap.

The second aim was to determine whether disgust propensity was associated most strongly with spider fear, while disgust sensitivity would be relatively more important in disorders where disgust-related preoccupations are predominant (i.e., obsessive compulsive disorders, blood phobia, eating disorders). Corroborating previous research (van Overveld et al., 2006), disgust propensity was associated with spider fear, while disgust sensitivity was associated with obsessive compulsive disorders and eating disorders. Both disgust traits were associated with fear of blood.

When entering the disgust traits simultaneously in the regression analysis, neither trait predicted fear of spiders, although the $p$-value for disgust propensity fell just outside the range of statistical significance. This is in line with earlier research indicating low correlations between disgust propensity and spider fear (de Jong \& Merckelbach, 1998). Disgust sensitivity did not correlate with spider fear, which confirms earlier work that fear is the dominant emotion in 
spider phobia (Tolin, Lohr, Sawchuk, \& Lee, 1997). So, a generally enhanced readiness to experience disgust does not seem to contribute to the etiology of spider distress.

In line with earlier studies, disgust propensity appeared related to fear of blood (de Jong \& Merckelbach, 1998; Olatunji, Sawchuk, de Jong, \& Lohr, 2006). Underlining the importance of measuring both disgust traits in phobic complaints, disgust sensitivity was independently associated with fear of blood. Extending previous reports, disgust sensitivity was also associated with obsessive compulsive complaints and eating styles Emotional Eating and External Eating. Further, a border-significant effect for disgust propensity to predict scores on the VOCI supports earlier work that found elevated disgust propensity in people with obsessive compulsive symptoms (Thorpe, Patel, \& Simonds, 2003). The data also revealed that disgust sensitivity may be even more important in obsessive compulsive disorders.

Earlier work showed that patients with eating disorders reported higher levels of disgust towards specific stimuli, such as food of animal origin (Davey et al., 1998; Troop, Treasure, \& Serpell, 2002). Moreover, eating disorder patients displayed elevated levels of disgust propensity compared to a clinical control group (Davey et al., 1998). Contrary to these findings, the present data indicated no relationship between eating disorders and disgust propensity. It could be that conceptual overlap in earlier research (e.g., Davey et al., 1998) may have confounded results since indices on disgust propensity (i.e., DS) and the Eating Attitudes Test (EAT; Garner \& Garfinkel, 1979) both contain items on food. Disgust sensitivity did correlate with eating styles, which sustains the idea that disgust sensitivity may be more strongly associated with some disorders than disgust propensity (van Overveld et al., 2006).

Additionally, as suggested by Davey (2003), scales were included on neuroticism and a seemingly disgust-unrelated disorder (depression). In line with earlier work (Davey \& Bond, 2006; Olatunji, 2006), the association between disgust propensity / sensitivity and symptoms of psychopathology was independent of trait negative affectivity. Although associations with disgust propensity and psychopathological symptoms were restricted to disgust-related forms of psychopathology, disgust sensitivity also appeared associated with a disgust-unrelated condition (i.e., depression). However, as the ZSDR and DPSS-RS both emphasize negative and catastrophic thinking, it is conceivable that this may present a shared characteristic. Future research should investigate the specificity of the DPSS-R further by including indices on psychopathology that do not contain such overlap.

Several issues remain open for further investigation. First, the research population was restricted to "healthy" students. Given the potential importance of the traits to psychopathology, the alleged differential role of disgust propensity and sensitivity should also be tested within clinical samples.

Second, a cross-sectional design was used. Thus, it remains to be established whether disgust propensity and sensitivity pose risk factors for developing psychopathology or are mere epiphenomena of these conditions. Third, the DPSS-R does not contain conceptual overlap with psychopathology indices, but does share items with the Anxiety Sensitivity Index (ASI; Reiss et al., 1986). For example, both contain items on 'fear of feeling nauseous'. To ensure that disgust and anxiety sensitivity present separate constructs, future research should investigate the relationship between these indices. 


\section{CHAPTER 6}

To conclude, disgust propensity and sensitivity appeared differentially related to psychopathology. As expected, disgust propensity was associated most strongly with spider fear whereas disgust sensitivity was most strongly associated with obsessive compulsive disorders and eating disorders. Both traits appeared associated with fear of blood. Next, it should be determined whether elevated levels of disgust propensity and sensitivity are risk factors for developing certain clinical complaints and whether disgust sensitivity could be more important in the etiology of certain disorders (e.g., blood phobia, obsessive compulsive disorders, eating disorders). 


\section{Chapter 7}

\section{An internet-based study on the relation between disgust sensitivity and emetophobia}




\section{CHAPTER 7}

\section{Abstract $^{\dagger+}$}

In the etiology of disgust-relevant psychopathology, such as emetophobia (fear of vomiting), two factors may be important: disgust propensity, how quickly the individual experiences disgust, and disgust sensitivity, how negatively does the individual evaluate this disgust experience (van Overveld et al., 2006). Hence, the current study examines whether emetophobic participants display elevated levels of disgust propensity and sensitivity, and whether these factors are differentially related to emetophobia. A group of emetophobic members of a Dutch website on emetophobia $(n=172)$, and a control group $(n=39)$ completed an internet survey containing the Emetophobia Questionnaire, Disgust Propensity and Sensitivity Scale-Revised, Disgust Scale, and Disgust Questionnaire. Results showed that the emetophobic group displayed significantly elevated levels of both disgust propensity and disgust sensitivity compared to the control group. Most importantly, disgust sensitivity consistently was the best predictor of emetophobic complaints.

\footnotetext{
${ }^{{ }^{\dagger}}$ Published as: van Overveld, M., de Jong, P. J., Peters, M. L., van Hout, W. J. P. J., \& Bouman, T. K. (2008). An internet-based study on the relation between disgust sensitivity and emetophobia. Journal of Anxiety Disorders, 22, 524-531.
} 
Although estimates on the prevalence of emetophobia (fear of vomiting) range between 1.7 $3.1 \%$ for men, and $6-7 \%$ for women (Philips, 1985; van Hout \& Bouman, 2006), only few empirical data are available regarding this peculiar disorder (Boschen, 2007). Emetophobia is defined as fear of vomiting and is frequently interpreted as a comorbid disorder in several forms of psychopathology, like social phobia (Marks, 1987), agoraphobia (Pollard, Tait, Meldrum, Dubinksi, \& Gall, 1996), or panic disorder (Lydiard, Laraia, Howell, \& Ballenger, 1986). However, it has been observed that emetophobia does not fit easily into any of these categories (Lelliott, McNammee, \& Marks, 1991). This observation is further sustained by case studies in which emetophobia appears the primary, rather than secondary diagnosis (e.g., Dattilio, 2003; Moran, \& Brien, 2005; Ritow, 1979), as well as the observation that the appearance of comorbid disorders starts only after the onset of emetophobia (Lipsitz et al., 2001).

The three most common central themes relevant to people characterized by emetophobia are fear of vomiting themselves, fear of seeing others vomit, or fear of vomiting in the presence of other people (Veale \& Lambrou, 2006; van Hout, \& Bouman, 2006; van Hout, Lansink, \& Bouman, 2005). People with emetophobia tend to avoid all stimuli that may even be slightly related to vomiting. This may range from avoiding the use of the word 'vomit' to eating in public, or even pregnancy out of fear of becoming nauseous (Massop, 2005).

As vomit itself is one of the few universally accepted disgust stimuli (Rozin, Haidt, McCauley, 2000), it seems reasonable to suspect that disgust and fear of contamination are somehow involved in emetophobia. Disgust may be involved in the etiology and maintenance of psychopathology in various ways. First, it has been argued that certain stimuli are characterized by high levels of contamination potency. Objects that are regarded highly disgusting, and that are capable of engaging physical contact with the individual, tend to be evaluated as having high contamination potency. For example, spider phobics consider spiders to be highly disgusting objects with a high contamination potency (Thorpe \& Salkovskis, 1998; Mulkens, de Jong, \& Merckelbach, 1996). Consequently, research indicated that indeed spider phobia may be better understood as a fear of physical contact and contamination with a disgusting object (de Jong \& Muris, 2002).

A second pathway in which disgust may be involved in psychopathology may be that some people are extremely sensitive to the experience of disgust and contamination. Recent work showed that for the development of psychopathology, it may not only be relevant how often people experience disgust (disgust propensity), but also whether they evaluate this experience negatively (i.e., disgust sensitivity; van Overveld, de Jong, Peters, Cavanagh, \& Davey, 2006). Recent reports indeed suggest that emetophobic individuals tend to avoid being disgusted, mostly in an attempt to avoid related feelings of nausea (Boschen, 2007; Lipsitz et al., 2001; Veale \& Lambrou, 2006).

The primary aim of the present study was to determine whether individuals with emetophobia display elevated levels of both disgust propensity and disgust sensitivity. The present study will therefore not only include more traditional disgust indices, namely the Disgust Questionnaire (DQ; Rozin et al., 1984) and Disgust Scale (DS; Haidt et al., 1994), but also the newly devised Disgust Propensity and Sensitivity Scale-Revised (DPSS-R; van Overveld et al., 2006). The latter is the only instrument to date that covers disgust sensitivity in addition 


\section{CHAPTER 7}

to disgust propensity. Similar to other disorders in which disgust is presumed to be involved, such as spider fear and blood fear (van Overveld et al., 2006), it was hypothesized that both levels of disgust propensity and disgust sensitivity would be elevated in emetophobic individuals compared to control participants. Additionally, recent findings indicated that disgust propensity and disgust sensitivity may be differentially involved in various types of psychopathology (van Overveld et al., 2006). Disgust sensitivity was found to be most strongly involved in disorders in which disgust rather than fear is the dominant emotion (e.g., blood phobia; van Overveld et al., 2006). On the basis of evidence suggesting that emetophobic individuals tend to avoid being disgusted, we hypothesized that enhanced disgust sensitivity would be more strongly involved in emetophobia than heightened levels of disgust propensity.

\section{Methods}

\section{Participants.}

All members $(N=348)$ of a website for people with emetophobic complaints were invited to participate in the present internet study through a newsletter via e-mail and via advertisements on the website www.emetofobie.nl and in newsletters to its members. However, not all people on the member list were still active members of the site. Furthermore, although most members of this particular website are characterized by emetophobic complaints, successfully treated emetophobics also tend to remain a member of the site. Therefore, it was stressed in the advertisements that in order to participate, one should still have emetophobic complaints. Additionally, all emetophobic participants were encouraged to invite someone in their close personal surroundings (e.g., relatives, friends, partner) to complete the survey as well. Participants were instructed that this person should preferably match the emetophobic participant in age and gender. Furthermore, it was emphasized that these people should be free of any emetophobic complaints. Of the emetophobic group, $32 \%$ were able to recruit a nonemetophobic participant. These control participants had to contact the experimenter themselves via email, to be able to participate. Consequently, both experimenter and participants were generally aware to which group the participants were assigned. Further, prior to the administration of the questionnaires, participants indicated whether they had a fear of vomiting (yes / no). However, although everybody was allowed to participate, participants did not know whether they genuinely qualified as emetophobic or as control, as this was generally determined only after completion of all questionnaires by comparing the self-diagnosis with group membership. Since $40 \%$ of all the members of the website participated, this resulted in a self-diagnosed emetophobic group ( $n=138 ; 16$ men, 122 women) with a mean age of 25.4 years ( $S D=8.2$; range: $15-68$ years). Of the emetophobic individuals, $4.5 \%$ completed only primary education (i.e., elementary school; approximately 6-8 years of education), $67.7 \%$ completed secondary education (i.e., high school; approximately 10-14 years of education), and $27.8 \%$ completed tertiary educational levels (i.e., college or university; approximately 14-20 years of education). Participants in the control group ( $n=43 ; 3$ men, 40 women) had a mean 
age of 24.7 years ( $S D=5.9$; range: $15-43$ years). Furthermore, $5.1 \%$ of the control group completed primary education, $59 \%$ completed secondary education, and $35.9 \%$ completed tertiary educational levels.

\section{Materials}

Disgust Propensity and Sensitivity Scale-Revised (DPSS-R; van Overveld et al., 2006). The 16item DPSS-R measures disgust propensity (i.e., to what extent do participants experience disgust in any given situation) and sensitivity (i.e., how horrid do participants consider this disgust experience). On a scale from 1 (= 'never') to 5 (= 'always'; range: 16-80), participants rate a series of positions on both how often they experience specific (bodily) symptoms related to disgust (e.g., 'I screw up my face in disgust.'), and to what extent these symptoms are upsetting to them (e.g., 'When I feel disgusted, I worry that I might pass out.'). The DPSS-R and its subscales appear internally consistent (alpha coefficients $>.71$; van Overveld et al., 2006). In the present study, internal consistency was high for both propensity and sensitivity (both alphas: .88).

Disgust and Contamination Sensitivity Questionnaire (DQ; Rozin, Fallon, \& Mandell, 1984); This 24-item questionnaire examines the tendency of the participants to reject desirable and perfectly edible food items when they have been contaminated with disgusting stimuli. On a scale from 1-9 (1 = 'not at all', 9 = 'very much'; range 24-216, where high scores reflect low disgust propensity), participants indicate their willingness to eat certain food items after it has been contaminated (e.g., 'Imagine a bowl of a particular type of soup that you like very much. How much would you like to eat that soup from a thoroughly washed used dog bowl?'). The DQ is internally consistent (alpha $=.72$ to .86 , Davey, 1994; .80, Mulkens et al., 1996).

Disgust Scale (DS; Haidt et al., 1994). The DS contains 32 items and examines the individual's disgust propensity, the tendency to experience disgust for eight types of disgust elicitors: Animals, Bodily Products, Body-Envelope Violations, Death, Food, Hygiene, Sex, and Sympathetic Magic. The first half of the DS has true/false type questions (e.g., ' I might be willing to try eating monkey meat under some circumstances.'), the second half of the DS has to be rated on a 3-point Likert-scale from 0 (= 'not disgusting at all') to 2 (= 'extremely disgusting') (e.g., 'You hear about an adult woman who has sex with her father.'). Thus, a total score (range: 0-32) as well as eight subscale scores can be obtained. Previous research indicates that although alphas for the subscales are low (.34-.64; Haidt et al., 1994), alpha is high for the total score (.84; Haidt et al., 1994).

Emetophobia Questionnaire (EQ; Bouman \& van Hout, in preparation). The EQ is a 115 -item scale developed within a larger research project in which the University of Groningen and Maastricht University participate (Bouman \& van Hout, in preparation). The questionnaire contains items on various aspects of emetophobia, such as worrying about vomiting, bodily sensations, fear of vomiting (e.g., 'I am afraid of becoming nauseous.'), the avoidance of vomitrelated situations (e.g., 'I avoid being around people who look as if they may be sick.'), and the consequences of emetophobia in daily life (e.g., 'Because of my fear of vomiting, I have lost weight.'). Items have to be rated on a Likert-scale from 1 (= 'not at all') to 5 (= 'very much'). 


\section{CHAPTER 7}

For the 16 items referring to the consequences of emetophobia, an additional answer category was added $(0=$ 'not applicable') for participants without emetophobic fears. In this study, four scales of this questionnaire were used: a total score (98 items, range: $98-575$; alpha $=.97$ ) and three a priori subscales on Emetophobic Complaints (68 items; range: 68-340; alpha $=.97$ ), Emetophobic Avoidance (30 items; scoring range: $30-150$; alpha $=.94$ ), and Emetophobia Consequences (16 items; scoring range: 0-80; alpha $=.96$ ).

\section{Procedure}

People who indicated to be willing to participate received an individual login number and password, with which they could log in to the University server. Once logged in, all participants completed a few biographical questions on age, sex, and educational level. In addition, they were asked to indicate whether they had a fear of vomiting. After this, participants received Dutch versions of the DPSS-R, EQ, DQ, and DS. Questionnaires were always administered in that particular order. The questionnaires were completed and analyzed anonymously. No reward was given upon completion of the questionnaires.

\section{Results}

\section{Descriptive statistics}

The results of two members of the website were excluded based on their self-diagnosis, on which they indicated that they did not have a fear of vomiting. The results of four participants from the control group were also excluded from further analyses, as they indicated that they had (some) fear of vomiting. Further, data of one person in the emetophobic group and two people in the control group were removed because they dropped out before completing $10 \%$ on the first two questionnaires, the EQ or the DPSS. Thus, a sample of 133 people in the emetophobia group and 39 people in the control group remained.

As the inclusion criterion to be assigned to either of the two groups relied solely on selfdiagnosis, it is important to establish whether the classification appears to be valid. This was done by studying the difference in scores on the EQ of the emetophobic group compared to the control group. Mean scores on the EQ indicated that indeed the emetophobic and the control groups scored very differently with respect to emetophobic complaints. Only the two highest scoring individuals of the control group (mean EQ-scores 228 and 261 respectively) had overlapping scores with the emetophobic group, and even they both fell within the first decile of the scores of the emetophobic group. Furthermore, one person in the emetophobic group scored considerably lower than the rest of the participants in the emetophobic group (EQ-score: 173, while the second lowest person scored 239), and it therefore could be questioned whether that person can be considered emetophobic on the basis of the EQ-scores. Nevertheless, the results of these persons were included in the group to which they assigned themselves. Additionally, the ROC-Curve provides a trade-off between true positive rate (sensitivity) and 
false positive rate (1 - specificity) of classified individuals. Based on this curve, a cut-off score can be abstracted for the EQ. Here, results showed that when placing the cut-off score at a score greater than or equal to 233.50, a sensitivity of .99 and a 1-specificity of .03 would be obtained. This would be very acceptable, and it confirms that our two groups appear distinct with regard to emetophobic complaints.

Next, mean scores on all questionnaires were calculated for both the emetophobic and the control group. Then, one-way ANOVAs were used to determine whether these scores differed significantly between groups. Table 1 shows that, as expected, the emetophobic participants were indeed characterized by significantly more emetophobic complaints and vomit-related avoidance behavior (as indexed by EQ-Complaints and EQ-Avoidance and EQ-Consequences). Furthermore, compared to the control group, the emetophobic group displayed significantly elevated levels of disgust propensity (as indexed by the DQ, DS, and DPSS-R-Propensity), as well as disgust sensitivity (as indexed by DPSS-R-Sensitivity).

Table 1.

Mean scores and standard deviations for the emetophobic and the control group

\begin{tabular}{lccccc}
\hline Indices & Emetophobic group & Control group & $F(1,171)$ & $P$ & $\eta^{2}$ \\
\hline EQ total & $360.21(64.23)$ & $176.54(29.24)$ & 299.27 & $<.001^{* *}$ & .64 \\
Complaints & $206.95(36.89)$ & $104.48(24.48)$ & 265.25 & $<.001^{* *}$ & .61 \\
Avoidance & $87.55(18.21)$ & $49.97(8.89)$ & 154.54 & $<.001^{* *}$ & .48 \\
Consequence & $47.75(14.19)$ & $.77(3.40)$ & 418.77 & $<.001^{* *}$ & .72 \\
DS total & $18.44(4.93)$ & $15.49(4.37)$ & 11.09 & $<.01^{* *}$ & .06 \\
DQ total & $107.76(38.87)$ & $138.00(35.08)$ & 18.86 & $<.001^{* *}$ & .12 \\
DPSS-R-Propensity & $25.18(4.98)$ & $18.54(4.01)$ & 58.27 & $<.001^{* *}$ & .25 \\
DPSS-R-Sensitivity & $25.44(5.28)$ & $13.41(3.64)$ & 176.99 & $<.001^{* *}$ & .51 \\
\hline Valid n & 133 & 39 & & &
\end{tabular}

Note. Standard deviations are described in parentheses; Abbreviations: Emetophobic Questionnaire (EQ), Disgust Scale (DS), Disgust Questionnaire (DQ), Disgust Propensity and Sensitivity Scale-Revised (DPSS-R).

** Significant at $p<.01$

\section{Correlations between disgust and emetophobia}

Table 2 displays the Bivariate Pearson's $p$ - $m$ correlations between the subscales of the EQ (EQComplaints and EQ-Avoidance) and the disgust indices (DPSS-R, DS, DQ) in the entire sample. All correlations between the level of emetophobic complaints (EQ-Complaints) and either disgust propensity (DQ, DS, DPSS-R-Propensity) or disgust sensitivity (DPSS-R-Sensitivity) were moderate to high and statistically significant. The correlations between EQ-Avoidance and all disgust indices revealed an identical pattern. The correlation between the DQ and the EQ was negative, yet, this is due to the scaling of the DQ (which has an inverse scoring format where low total scores reflect higher disgust propensity). 


\section{CHAPTER 7}

Table 2.

Correlations between the Emetophobia Questionnaire and the disgust indices.

Note: Abbreviations: Emetophobic Questionnaire (EQ), Disgust Scale (DS), Disgust Questionnaire (DQ), Disgust Propensity and Sensitivity Scale-Revised (DPSS-R)

** Significant at $p<.01$

\section{Contribution of disgust to emetophobia}

Hierarchical regression analyses (method = Enter) were performed to determine the relative contributions of disgust propensity and disgust sensitivity to emetophobia, and whether the disgust sensitivity component explains additional variance compared to disgust propensity. For these analyses, the scores on the EQ-Complaints or EQ-Avoidance were used as dependent variables. As predictor variables, scores on DPSS-R-Propensity, DPSS-R-Sensitivity, DQ-total scores and DS-total scores were used. All participants were included in these analyses. In order to investigate whether both disgust propensity and disgust sensitivity contribute uniquely to emetophobia, the disgust propensity indices (DQ, DS, and DPSS-R-Propensity) would be entered in the first step in each analysis, while the disgust sensitivity index (DPSS-R-Sensitivity) 
DISGUST IN EMETOPHOBIA

Table 3.

Hierarchical regression analyses for the disgust indices as predictors of the Emetophobia Questionnaire (EQ) and its subscales.

\begin{tabular}{|c|c|c|c|c|c|}
\hline & Questionnaire & $B$ & $S E B$ & $B$ & $p$ \\
\hline Analysis 1 & \multicolumn{5}{|c|}{ Step $1 R^{2}=0.55, F=63.38, p<0.01$} \\
\hline \multirow[t]{8}{*}{ EQ-total } & DQ & -.46 & .16 & -.19 & $<.01 * *$ \\
\hline & DPSS-RP & 10.66 & 1.15 & 61 & $<.01^{* *}$ \\
\hline & DS & .50 & 1.35 & .03 & .71 \\
\hline & \multicolumn{5}{|c|}{ Step $2 R^{2}=0.77, F=151.83, p<0.01$} \\
\hline & DQ & -.22 & .12 & -.09 & .07 \\
\hline & DPSS-RP & 1.50 & 1.11 & .09 & .18 \\
\hline & DS & -.78 & .98 & -.04 & .42 \\
\hline & DPSS-RS & 10.50 & .85 & .78 & $<.01 * *$ \\
\hline Analysis 2 & \multicolumn{5}{|c|}{ Step $1 R^{2}=0.55, F=66.03, p<0.01$} \\
\hline EQ- & DQ & -.26 & .09 & -.19 & $<.01 * *$ \\
\hline \multirow[t]{7}{*}{ Complaints } & DPSS-RP & 6.05 & .65 & .61 & $<.01^{* *}$ \\
\hline & DS & .54 & .76 & .05 & .48 \\
\hline & \multicolumn{5}{|c|}{ Step $2 R^{2}=0.76, F=138.89, p<0.01$} \\
\hline & DQ & -.12 & .07 & -.09 & .08 \\
\hline & DPSS-RP & 1.00 & .64 & .10 & .12 \\
\hline & DS & -.17 & .56 & -.02 & .77 \\
\hline & DPSS-RS & 5.80 & .49 & .75 & $<.01 * *$ \\
\hline Analysis 3 & \multicolumn{5}{|c|}{ Step $1 R^{2}=0.51, F=54.64, p<0.01$} \\
\hline EQ- & DQ & -1.03 & .04 & -.18 & $.01^{*}$ \\
\hline \multirow[t]{7}{*}{ Avoidance } & DPSS-RP & 2.24 & .28 & .54 & $<.01^{* *}$ \\
\hline & DS & .43 & .33 & .09 & .20 \\
\hline & \multicolumn{5}{|c|}{ Step $2 R^{2}=0.66, F=73.43, p<0.01$} \\
\hline & DQ & -.06 & .03 & -.10 & .10 \\
\hline & DPSS-RP & .44 & .31 & .11 & .17 \\
\hline & DS & .17 & .28 & .04 & .53 \\
\hline & DPSS-RS & 2.07 & .24 & .65 & $<.01^{* *}$ \\
\hline Analysis 4 & \multicolumn{5}{|c|}{ Step $1 R^{2}=0.40, F=35.22, p<0.01$} \\
\hline EQ- & DQ & -.12 & .05 & -.21 & $<.01 * *$ \\
\hline \multirow[t]{7}{*}{ Consequences } & DPSS-RP & 2.38 & .32 & .56 & $<.01^{* *}$ \\
\hline & DS & -.47 & .38 & -.10 & .22 \\
\hline & \multicolumn{5}{|c|}{ Step $2 R^{2}=0.66, F=117.77, p<0.01$} \\
\hline & DQ & -.06 & .04 & -.10 & .10 \\
\hline & DPSS-RP & -.01 & .33 & -.00 & .98 \\
\hline & DS & -.80 & .29 & -.17 & $<.01^{* *}$ \\
\hline & DPSS-RS & 2.74 & .25 & -.83 & $<.01^{* *}$ \\
\hline
\end{tabular}

Note: Abbreviations: Emetophobic Questionnaire (EQ), Disgust Scale (DS), Disgust Questionnaire (DQ), Disgust Propensity and Sensitivity Scale-Revised Propensity (DPSS-RP), Disgust Propensity and Sensitivity Scale-Revised Sensitivity(DPSS-RS). Significant at $p<.05 ; * *$ Significant at $p<.01$ 


\section{CHAPTER 7}

\section{Discussion}

The main findings can be summarized as follows: i) the emetophobic group demonstrated elevated levels of both disgust propensity and disgust sensitivity, compared to the control group, ii) there was a strong interrelationship between the intensity of emetophobic complaints on the one hand and the levels of disgust propensity and disgust sensitivity on the other, and iii) most important, disgust sensitivity proved consistently to be the single best predictor of the variance in scores on the EQ.

As expected, it was found that emetophobic individuals demonstrate more symptoms of vomit-related concerns than the control group. The emetophobic individuals were characterized by significantly more emetophobic complaints (e.g., worrying about vomiting or seeing others vomit), avoid vomit-related stimuli (e.g., the use of the word 'vomit' or standing near a drunk person) significantly stronger, and reported that fear of vomiting has a stronger impact on their daily life (e.g., experiencing guilt or shame) compared to the control group.

Furthermore, it was hypothesized that disgust may be involved in emetophobia. Recently, it was shown that for disgust-relevant complaints to emerge, it may not only be important how quickly and how often people experience disgust (disgust propensity), but more so whether they evaluate this experience negatively (i.e., disgust sensitivity; van Overveld et al., 2006). The present findings confirm the view that disgust sensitivity appears involved in disgustrelevant complaints. Most importantly, upon studying the relation between disgust sensitivity, disgust propensity and the Emetophobia Questionnaire, disgust sensitivity proved the single best predictor of the variance in scores on the EQ. Thus, from the data from the present research, it can be concluded that disgust sensitivity contributes more strongly than disgust propensity to emetophobia. This strengthens the previous postulation that the differential role of disgust propensity and disgust sensitivity may vary across disorders (van Overveld et al., 2006).

Future research should take several issues into account. First, research on emetophobia is presently lacking a standardized instrument to establish emetophobia. Thus, most studies use self-developed questionnaires (e.g., Bouman \& van Hout, 2006; Lipsitz et al., 2001; Veale, 2006). Consequently, the psychometric properties of these indices remain to be determined. This is particularly problematic, as this renders a comparison between studies more difficult. Future research should focus on developing a valid and reliable instrument for establishing emetophobic concerns.

Secondly, the present study did not use clinically established patients, but relied on selfdiagnosis. However, most members of the site are treatment-seeking individuals, persons who have been in treatment or who are currently embarking in treatment. Moreover, results confirmed that the emetophobic group were indeed characterized by significantly elevated levels on a broad range of emetophobic concerns. This adds validity to the claim that these groups are indeed different with regard to emetophobic complaints. Nevertheless, it remains to be determined whether clinical populations would display similar elevated levels. Thus, future research should investigate whether similar results can be obtained in a sample comprised of patients with an established clinical diagnosis of emetophobia, compared to a non-clinical 
control group. Germane to this issue, the present study compared only non-clinical individuals to a self-diagnosed clinical group. Since the present study did not include a clinical comparison group with disgust-unrelated psychopathology, it remains to be seen whether disgust sensitivity has a unique relationship with vomiting fears. An important next step would be to test the specificity of the relationship between emetophobia and disgust sensitivity, for example, by including clinical comparison groups (e.g., patients with disgust-unrelated psychopathology such as depression or chronic pain patients).

Thirdly, in line with earlier studies, the gender distribution of emetophobic complaints was extremely skewed. Previously, an internet survey by Lipsitz et al. (2001) revealed that $89 \%$ of the emetophobic individuals were women, Veale \& Lambrou (2006) found $97 \%$ of the emetophobic sample in his study to be women. Emetophobia appears to be found predominantly in young women who are relatively high educated. One explanation for this could be that disgust propensity is crucially involved. Research indicates that women are characterized by elevated levels of disgust propensity (Matchett \& Davey, 1991; Ware et al., 1994), and that this may explain why disgust-relevant psychopathology is observed predominantly in women (Olatunji, Arrindell, \& Lohr, 2005; Olatunji, Sawchuk, Arrindell, \& Lohr, 2005). Even so, compared to contamination-related OCD, or other disgust-relevant disorders, such as blood phobia, the gender distribution of emetophobia appears extremely skewed. Alternatively, women may just be more interested in websites on health issues than men (Buchanan, \& Smith, 1999), and search for them more actively. Additionally, since women in this study were young, they may simply have better accessibility to the internet. For example, $70 \%$ of people between 20 - 50 years use the internet, whereas this number decreases dramatically in older people (Hayslett, \& Wildemuth, 2004). Yet, using non-web-based studies, it was still reported that people with vomit phobias are young women (Himle, McPhee, Cameron, \& Curtis, 1989; Veale \& Lambrou, 2006). However, as the number of studies is scarce at present, future studies should determine whether the skewed age and gender distributions are consistently observed in emetophobia.

Fourth, if disgust sensitivity indeed uniquely contributes to emetophobia, it would be interesting to investigate whether inflated levels of disgust sensitivity are merely an epiphenomenon of emetophobia or whether there may be a causal relationship. One way to investigate this issue would be to conduct a longitudinal study, in which participants who are free of any disgust-relevant psychopathological complaints but who are characterized by either high or low levels of disgust sensitivity at the start of the study will be measured for a number of years. As the age range of 13-17 years appears the critical period in which emetophobic complaints start for most emetophobics (Lelliot et al., 1991; van Hout \& Bouman, 2006; van Hout, Lansink, \& Bouman, 2005), it would be advisable to study participants during this vulnerability period to examine whether increased levels of disgust sensitivity render emetophobic or other disgust-relevant complaints (such as spider phobia, blood phobia, contamination-related $\mathrm{OCD}$ ) more likely to develop.

Additionally, the current study does have certain apparent limitations. Firstly, the current study relied fully on self-report indices. Moreover, as participants were generally aware of their group membership, the observed relationship between disgust propensity, disgust sensitivity 


\section{CHAPTER 7}

and emetophobia may thus have become inflated. Therefore, it should be investigated whether similar relationships can be found when another type of research methodology is applied, for example, whether emetophobic individuals display disgust-relevant physiological reactivity upon confrontation with vomit-related stimuli compared to healthy controls (e.g., activity of the disgust specific $\mathrm{m}$. levator labii; de Jong, Peters, \& Vanderhallen, 2002) or are characterized by stronger disgust and contamination related associations for vomit-related situations and stimuli (e.g., on the Implicit Association Test). Secondly, as the present study is cross-sectional, no causal inferences can be made. Thus, although it was concluded that disgust sensitivity contributes stronger to emetophobia than disgust propensity, it remains to be determined whether inflated levels of disgust sensitivity are causally related to emetophobia, or are merely an epiphenomenon of fear of vomiting.

In sum, the present findings further sustain the notion that there may be a distinct subgroup of patients with an intense fear of vomiting (e.g., Lipsitz et al., 2001; Veale \& Lambrou, 2006). Most importantly, the present findings extended earlier work by demonstrating that disgust sensitivity, rather than disgust propensity, may be involved in emetophobia. Nevertheless, future studies could benefit significantly from both the development of a valid and reliable index for emetophobia, and the inclusion of patients with emetophobia and a clinical comparison groups. 


\section{Chapter 8}

Digestive and cardiovascular responses to core and animal-reminder disgust 


\section{CHAPTER 8}

\section{Abstract}

The two-stage model of disgust differentiates between core and animal-reminder (A-R) disgust (Rozin \& Fallon, 1987). This study investigates whether core and A-R disgust elicit distinct physiological reaction patterns. Further, in line with the idea that A-R disgust is critically involved in blood phobia and may explain typical phenomenology of psychopathological symptoms (e.g., dizziness), we investigated whether physiological patterns (if present) would differ specifically for A-R disgust between high and low blood fearful participants. Therefore, high $(n=30)$ and low $(n=30)$ blood fearful individuals engaged in guided imagery of core disgust, A-R disgust, and neutral stimuli. Overall, both disgust scripts lead to increased activity in the digestive component of the autonomic nervous system (ANS). For cardiac components, sympathetic activity decreased, whereas no parasympathetic reactivity was observed compared to the neutral script. No differences were observed in physiological reactivity between the A-R and core disgust scripts. Meanwhile, in line with the idea that disgust is involved in blood phobia, subjective symptoms of vomit and dizziness did differentiate between high and low blood fearful participants, as subjective symptoms were most pronounced in the high blood fearful group. Contrary to our expectations, increases in subjective symptoms were apparent for both disgust types and not specifically for A-R disgust. So, physiological reactivity appeared relatively independent of type of disgust elicitor which, in turn, may reflect a general hard-wired protective mechanism to prevent contamination with pathogens. 
From an evolutionary perspective, disgust is seen as a defensive mechanism protecting the organism from contamination by pathogens (Rozin \& Fallon, 1987). Accordingly, disgust is focused on the intersection between the body and the environment and concentrates on the skin and body apertures (Rozin et al., 1995; Fessler \& Haley, 2006). The strength of the disgust response increases as a function of proximity of the potential contaminant and the sense of inclusion. Thus, the closer the stimulus and/or the more intense the physical contact, the stronger is the disgust response. Core disgust is exemplified by food-rejection responses, reactions that occur at the threat of consuming a disgusting stimulus (Rozin \& Fallon, 1987; Power \& Dalgleish, 1997).

In his two-stage model of disgust, Rozin and colleagues argued that the defensive mechanism of disgust originally evolved to prevent the body from contamination by pathogens and toxins from the outside environment (core disgust) is extended to stimuli and/or behaviors that remind us of our animal nature (Rozin et al., 1999). Accordingly, people are not only disgusted at the prospect of having to consume something disgusting, they also tend to be disgusted by stimuli that emphasize the similarities between humans and animals. This reminder that people are of animal origin is a thought that most people consider unacceptable (Rozin et al., 1999), presumably because humans wish to rise above the animal kingdom, and not be reminded that they too are mortal creatures of flesh and blood. Therefore, disgust for these stimuli is labeled animal-reminder disgust (A-R; Haidt et al., 1994).

Consistent with the two-stage disgust model of Rozin et al. (1999), factor analytical studies have shown that core disgust and animal reminder disgust elicitors can be systematically distinguished as separate constructs (Olatunji et al., 2007). Moreover, there is accumulating evidence that disgust is involved in various psychopathological complaints (Olatunji \& McKay 2007). Adding to the validity to differentiate between A-R and core disgust, a series of studies provided evidence that $A-R$ and core disgust are differentially involved in these various types of psychopathological symptoms. For example, fear of spiders is typically associated with heightened levels of core disgust propensity (i.e., tendency to experience core disgust more rapidly), whereas fear of blood-injury stimuli has been found to be specifically associated with a heightened disgust propensity for A-R stimuli (de Jong \& Merckelbach, 1998). More recently, Sawchuk et al. (2000) corroborated these earlier findings showing that blood-injection phobics displayed a stronger disgust propensity for A-R stimuli than spider phobics or non phobics, whereas no such difference was evident for core disgust stimuli.

The major aim of the present study was to establish whether core and animal-reminder disgust may not only vary in their reference to different classes of disgust elicitors, but perhaps also in their concomitant response patterns of the autonomic nervous system (ANS). If so, this would not only further substantiate the validity of distinguishing between different types of disgust, it may also help explaining the typical phenomenology of psychopathological symptoms that are related to each of these types of disgust.

For example, blood phobia is typically characterized by dizziness and feeling faint (Page, 1994), whereas these symptoms are usually absent in other disorders in which disgust is also assumed to be critically involved such as spider phobia. One explanation might be that specifically confrontation with A-R disgust stimuli (e.g., blood) results in a sympathetic 


\section{CHAPTER 8}

withdrawal and/or parasympathetic activation of cardiovascular components of the ANS resulting in a drop in blood pressure which, in turn, may give rise to feelings of dizziness. Indeed, blood- and injection stimuli have been found to lead to the so called diphasic response (i.e., initial increase in sympathetic nervous system (SNS) activity, followed by a sudden and possibly parasympathetically mediated drop below baseline levels). Most importantly, this response was most pronounced in blood fearful persons with relatively high levels of disgust propensity (Page, 2003).

Since core disgust originates from a withdrawal response regarding the oral incorporation of potential contaminants (Rozin et al., 1999), it would make sense that core disgust typically activates digestive rather than cardiovascular components of the ANS (e.g., saliva production, tendency to vomit). This may explain why feelings of dizziness are generally absent in psychopathological symptoms in which core disgust rather than A-R disgust is assumed to be critically involved (e.g., spider phobia; de Jong \& Merckelbach, 1998). Germane to the alleged role of the digestive nervous system in food aversion responses, it has been shown that consuming aversive bitter tasting foods increases saliva production (Norris et al., 1984). Such increased saliva production is closely associated with a gaping response or vomiting (Hornby, 2001) and is thus likely to be the result of experienced disgust or nausea.

We decided to use guided imagery, because it might well be that using emotionally more intense movie clips or behavioral experiments may elicit 'fight / flight'-responses that interfere with the generation of straightforward disgust responses. That is, the 'fight / flight'-response is associated with an increase in sympathetic and parallel decrease in parasympathetic reactivity (Barron \& van Loon, 1989; McDougall, Widdop, \& Lawrence, 2005). It has been proposed that disgust is associated mainly with parasympathetic activity (Levenson, 1992). Since increases in sympathetic activity are generally associated with fear and anxiety, it is reasonable to assume that emotionally intense stimuli that evoke stronger sympathetic activity will reduce the sensitivity of the procedure to detect genuine disgust-induced (para)sympathetic response patterns. Imagination procedures seem less susceptible to such undesirable influences as participants remain in control over their emotional regulation, yet, they are nevertheless effective in evoking emotions (Lang, 1979; Prkachin, Williams-Avery, Zwaal, \& Mills, 1999, Vrana, 1993). Moreover, confrontation with a stressor may activate particularly sympathetically mediated activity in the cardiovascular system (Barron \& van Loon, 1989). Since we were interested in parasympathetic influences on the cardiovascular system, imagery was chosen as it may limit the effects of sympathetically mediated activity on this system.

To examine whether distinct disgust types evoke separate physiological response patterns, the study focused on core and A-R disgust elicitors. Core disgust imagery was hypothesized to induce activity of the digestive component of the ANS (increase in saliva production), whereas A-R disgust imagery was hypothesized to elicit reactivity of the vascular component (characterized by SNS withdrawal and PNS activation as indexed by increased T-wave amplitude, increased activity in the high frequency power band of heart rate variability, and a decrease in mean arterial pressure).

Further, given the predominant role of disgust in blood phobia (e.g., Page, 1994), we tested whether blood fearful participants and non blood fearful participants would differ in their 
subjective and physiological responding to core and $A-R$ disgust elicitors. On the basis of previous findings that blood fearful individuals typically display inflated levels of $A-R$ disgust propensity (e.g., de Jong \& Merckelbach, 1998), we anticipated that high blood fearful individuals would report more intense feelings of disgust and heightened physiological reactivity in response to A-R imagery than to core imagery compared to low blood fearful individuals (in particular, increased T-wave amplitude, increased activity in the high frequency power band of heart rate variability, and a decrease in mean arterial pressure). There are only a few studies in the literature that examined the peripheral physiological reactivity toward disgusting stimuli, and all of these studies used blood-related stimuli such as cardiac surgery clips (e.g., Palomba et al., 2000). It is therefore difficult to decide on the basis of these earlier findings whether these responses are specific for blood-related stimuli or are also representative for A-R disgust or disgust in general. Especially, since blood fearful individuals report to experience both fear and disgust for blood stimuli (e.g., Lumley \& Melamed, 1992; Tolin et al., 1997; Ritz et al., 2005). Therefore, an A-R script was used without references to blood stimuli to avoid confounding by fear-induced physiological reactivity. Reactivity during core disgust imagery was assumed to be similar for both groups (in particular, increased production of saliva and increased activity in the $\mathrm{m}$. levator).

\section{Methods}

\section{Participants.}

The participants were students at Maastricht University, from the faculties of Health Sciences, Medicine, and Psychology. The participants were recruited via posters, and advertisements in the university buildings and asked to complete the Blood Subscale of the Medical Fear Survey (MFS; Kleinknecht et al., 1996). From a total of 246 respondents, the thirty lowest and thirty highest blood-fearful individuals were invited to participate in the present experiment. Since most of the students at these faculties are women, the research population also consists predominantly of women $(86.7 \%)$. The mean age was 22.9 years $(S D=6.86)$. Participants were assigned to the high blood fearful group, if two of the following criteria were met: a) they rated themselves at least 7 on a scale from 1 (= not blood fearful at all) to 10 (=extremely blood fearful), b) MFS-Blood > 5, and c) Blood-Injury Phobia Questionnaire-Fear subscale (BIQFear; de Jong \& Merckelbach, 1998) > 20. Participants were considered low blood fearful if they: a) rated themselves non-blood fearful $(<5)$, b) MFS-Blood $<5$, c) BIQ-Fear $<18$. In the high blood fearful group, $90 \%$ were women ( 27 women and 3 men) whereas in the low blood fearful group, $83 \%$ were women ( 25 women and 5 men). 


\section{CHAPTER 8}

\section{Materials}

\section{Questionnaires on fear of blood}

Multidimensional Blood / Injury Phobia Inventory (MBPI; Wenzel, \& Holt, 2003): This questionnaire was devised to measure the full range of phobic fears in blood phobia. The MBPI assesses four types of stimuli (injections, injury, hospitals, blood) and five types of responses (fear, avoidance, worry, fainting and disgust), and a self versus other focus. It consists of 40 items on a 5 -point scale ( $0=$ very slightly or not at all; $4=$ extremely). Based on factor analysis, six subscales have been identified in the MBPI: Injections, Hospitals, Fainting, BloodSelf, Injury and Blood-Injury-Others (Wenzel \& Holt, 2003). Internal consistency is good (Cronbach's alpha $=.91)$.

Medical Fear Survey (MFS; Kleinknecht et al., 1996); This questionnaire assesses fear of medical stimuli using five 10-item subscales (Mutilation, Sharp Objects, Examinations and Symptoms as Intimation of Illness, Injections and Blood Draws, Blood). Participants rate on a scale from 0 (= very slightly) to 4 (= extremely) whether a series of statements are typical of them. The subscales are reliable $(a=.84-.94$, Kleinknecht et al., 1999; $.87-.97$, present study).

Blood-Injury Phobia Questionnaire (BIQ); This is a 20-item questionnaire on which participants rate their fear of blood-injury items on a five-point Likert scale from 1 ('no fear') to 5 ('extreme fear'). The first ten items are derived from the Fear Questionnaire (Marks \& Mathews, 1979) and the Fear Survey Schedule (Wolpe \& Lang, 1964). The second part of the BIQ assesses if participants have fainted in the presence of the stimuli which were mentioned in the first ten items. Participants have to rate if they have fainted on a scale from 1 (= never) to 3 (= often). The internal consistency of the BIQ is good (.84; Merckelbach et al., 1999).

\section{Disgust Questionnaires}

Disgust Scale (DS; Haidt et al., 1994). The Disgust Scale measures disgust propensity across a broad domain of disgust elicitors. The 32 items can be divided into two parts. The first part of the DS has only true/false type questions on which the person has to fill in if they agree to a series of statements, the second part consists of 0-2 ratings of how disgusting specific experiences would be to them. While Cronbach's alpha for the total score is high (.84; Haidt et al., 1994), internal consistency for the original eight subscales is low (alphas ranging between .34-.64; Haidt et al., 1994). Therefore, the present study will use the two subscales that were recently recommended measuring only core disgust and A-R disgust (Olatunji et al., 2006).

The Disgust and Contamination Sensitivity Questionnaire (DQ; Rozin et al., 1984); This 24item questionnaire measures disgust propensity for core disgust. On a scale from 1-9 (1 = not at all, 9 = very much) participants have to rate how much they would like to eat a certain food item, after it has been contaminated with a specific item. A total score can be calculated (range 24 - 216) with an inverse scoring format, so higher scores reflect lower levels of disgust 
propensity. The DQ appears a reliable instrument (Cronbach's alpha = 80; Mulkens et al., 1996; .84, Merckelbach et al., 1993). Internal consistency ranges from .72 to .86 (Davey, 1994).

Disgust Propensity and Sensitivity Scale-Revised (DPSS-R; van Overveld et al., 2006). The DPSS-R is a measure of disgust propensity (i.e., the tendency to experience disgust more readily), and disgust sensitivity (i.e., how negatively does the individual evaluate the disgust experience). It measures these constructs irrespective of specific disgust elicitors. Participants have to rate on 16 items how often they experience certain bodily symptoms and their emotional impact on a scale from 1 (= 'never') to 5 (= 'always'; range: 32 - 160) (e.g., 'When I notice that I feel nauseous, I worry about vomiting'). A recent validation indicates that the DPSS-R is a valid and reliable instrument (alpha's for both subscales $>.71$; van Overveld et al., 2006).

\section{Apparatus}

Saliva production; The production of saliva was used as an index of parasympathetic activity related to the digestive system (Proctor \& Carpenter, 2007) and was measured using dental cotton rolls. One roll was placed sublingually and two rolls buccally, which is known as the Strongin, Hinsie \& Peck (SHP) technique (White \& Sher, 1977). The cotton rolls were inserted prior to the start of each script and replaced immediately after the presentation of each imagination script. The weight of the rolls was then determined before and directly after presentation of the imagination scripts. This method has been shown to be valid and reliable (Nederkoorn et al., 1999).

Facial ElectroMyoGraphy (EMG); As a physiological index of disgust, facial EMG at the levator labii and $\mathrm{m}$. corrugator supercilii were recorded. The levator muscle was selected because previous studies indicated that relative to other negative emotions, levator activity is specific to disgust (e.g., Vrana, 1993). The corrugator was selected because it was found to increase during negative emotions in general. Hereto, two pairs of $\mathrm{Ag} / \mathrm{AgCl}$ Beckman electrodes with a diameter of $0.25 \mathrm{~cm}$ and filled with Spectra 360 electrode gel were attached to the left side of the face, in accordance with recommendations by Fridlund and Cacioppo (1986).

ElectroCardioGram (ECG); the ECG-signals were recorded using pre-gelled single-use disposable $\mathrm{Ag} / \mathrm{AgCl}$ ECG surface electrodes (Ambu Bluesensor) with a diameter of $.55 \mathrm{~cm}$. Electrodes were placed on the participant's chest, two attached to the left lower ribs, and one electrode on the participant's sternum. Three unipolar leads connected the electrodes directly with a Picker/Schwarzer Coupler (ED14/12). Heart rhythm was continuously monitored and the data was stored on the lab server. From the ECG, HRV, and T-wave amplitude per script were derived off-line. Respiration rate was not taken into account in the present study.

Blood pressure; the blood pressure was recorded continuously with the vascular unloading method by using a Fin-a-press tonometer (TNO Biomedical Instruments). This device derives the systolic and diastolic blood pressure non-invasively from a pulse wave on a beat-by-beat basis by placing a cuff around the first finger (pointing finger) of the non-dominant hand. Arterial volume is measured using a servo-controlled photoplethysmograph. Prior to the start of the experiment, the Fin-a-press tonometer was calibrated for each participant. To prevent 


\section{CHAPTER 8}

confounding effects of skin temperature and movement, participants were asked to place the hand with the fin-a-press cuff on a towel and were instructed not to move their arm during the imagery.

Skin conductance level (SCL); the skin conductance level was recorded using a Schwarzer EDA48 Amplifier using the method of constant voltage (.5 volts). Two $0.70 \mathrm{~cm} \mathrm{Ag/AgCl} \mathrm{surface}$ electrodes were filled with isotonic paste and attached to the palmar side of the middle phalanges of the second and third fingers of the non-dominant hand.

EMG and ECG signals were fed through a Picker/Schwarzer Coupler (ED14/12) 12-channel EEG measurement amplifier to a PXI data-acquisition system (PXI 4472; National Instruments) using a low pass filter with a time constant of $0.3 \mathrm{~s}$. A $1000 \mathrm{~Hz}$ sample frequency was used. Further, possible $50 \mathrm{~Hz}$ interference was eliminated using a notch filter. Additionally, a high pass filter of $1.60 \mathrm{~Hz}$ and a low pass filter of $300 \mathrm{~Hz}$ were used. Skin conductance and blood pressure were fed through a $500 \mathrm{~Hz}$ anti-aliasing filter directly to the PXI data acquisition system. All data were recorded and stored using the lab server for further off-line processing.

\section{Imagination scripts}

Participants were asked to imagine as vividly as possible the following scripts: neutral, core disgust, and A-R disgust (see the Appendix for a full description of all scripts). A prerecording was made for all scripts while being read by an experimenter. They were stored as audio files (16 bit wav-files) on the lab computer (Dell GX270, Pentium 4A, 3 GHz, Windows 2000 Service Pack 4) which the experimenter could control to enable a synchronized presentation and data acquisition. The scripts were presented via speakers. In earlier research in our lab, these scripts successfully induced disgust (e.g., de Jong, Peters, \& Vanderhallen, 2002).

Visual Analog Scale (VAS)

Five VASs were administered. Participants rated on a $100 \mathrm{~mm}$-line their ability to imagine the scripts, whether they had experienced fear, disgust, dizziness, and a tendency to vomit during the scripts.

\section{Procedure}

The experiment was conducted at the Department of Clinical Psychological Science in a 'soundproof' room, separated from the data-recording lab by means of a 'one-way' screen. To avoid confounding effects by caffeine or other food substances on blood pressure, participants were instructed not to eat or drink anything in the two hours prior to the experiment. On arrival at the lab, participants had to provide informed consent following a brief introduction and completed a package of questionnaires, containing the MBPI, MFS (full version), BIQ, DS, DQ, and the DPSS-R. After this, the participant was hooked up to all physiological instruments. Given the potential risk of vasovagal fainting to occur during the experiment (in particular in the high fearful group during A-R imagery), the experimenter was not blind to group membership 
of the participants. To minimize the influence of eye-blink responses on the EMG signals and to make sure that the participants fully concentrated on the content of the scripts presented, the participants were instructed to close their eyes during the imagination procedure. Before presenting the scripts, participants received a five minute resting period in order to habituate to the laboratory setting. After dimming the lights, the scripts were presented. Each script lasted about five minutes. Before and after each script, participants completed the VASs and were asked to rinse their mouth with a sip of water. To minimize the influence of undesirable carry over effects we presented the scripts as follows: neutral script, core disgust script or A-R script (the order in which these two disgust scripts were presented was counterbalanced). After this, all electrodes and cuffs were removed and the participant received a small financial incentive (10 euros) for participating.

\section{Data reduction}

First, physiological data for EMG signals were digitally rectified, and integrated with a time constant of $0.3 \mathrm{~s}$ using specifically designed software programs. Mean levator labii and corrugator activity during each of the scripts was calculated. Similarly, for systolic and diastolic blood pressure and skin conductance level, mean levels during each script were determined. If values were outside the normal range ( $\geq 3$ SDs) for systolic blood pressure, diastolic blood pressure, and skin conductance, signals were visually inspected and possibly rejected as artefacts. Next, in line with recommendations by Shapiro et al. (1996), mean arterial pressure (MAP) was calculated using the following formula: DBP + $1 / 3$ (SBP - DBP).

The ECG signal was analyzed off-line with specifically designed software to detect R-waves and the various components comprising the T-wave amplitude $(\mathrm{P}, \mathrm{Q}$, and R-waves). The Twave amplitude was computed by the difference in $\mu \mathrm{V}$ between the maximum value included in a 100-300 ms window after the R-wave and the mean value of the 40-ms isoelectric line between P-wave and Q-wave (Rau, 1991). If the software could not identify a P, Q, or R-wave, the signal was rejected as an artefact. Next, the mean amplitude during the scripts was calculated. The T-wave amplitude indicates a withdrawal of sympathetic activity (Kline et al., 1998).

Heart rate variability was defined as the variability of the intervals between successive heart beats (R-tops of the ECG). Usually, three components are distinguished: low frequency, middle frequency and high frequency. The high frequency component is under the influence of the nervus vagus, and therefore is the most likely index for parasympathetic activity (Task Force of the European Society of Cardiology and the North American Society of Pacing Electrophysiology, 1996). Therefore, only the power in this High Frequency band (HF: .15 - .40 Hz) was studied in this experiment. The CARSPAN software package (version 2.11; IEC ProGamma, Groningen, the Netherlands) was used to correct the Inter-Beat-Intervals (IBI) and calculate the power in the $\mathrm{HF}$ band by means of spectral analysis on the IBIs according to a discrete Fourier Transform algorithm. CARSPAN was also used to correct possible artifacts in the ECG signals by replacing artefactual data by estimating an interpolation from both preceding and succeeding intervals. All data were visually inspected to ensure adequate corrections. After obtaining the HF band of 


\section{CHAPTER 8}

heart rate variability, in accordance with recommendations by the Task Force of the European Society of Cardiology and the North American Society of Pacing Electrophysiology (1996), normalized units were calculated for the power in the HF-band by means of the following formula: HF power / (total power - VLF) x 100. This was done for each script.

Due to technical difficulties, blood pressure was not measured in three persons in the nonblood fearful group, and for one non-blood fearful individual all physiological data were lost. Further, for one person in the blood fearful group, data for corrugator and levator was only partially recorded whereas for two others in that group, data for heart rate variability were not available. For one of these, all cardiac data were unavailable.

\section{Statistical analyses}

Missing values were observed only on the MFS and DQ $(<1 \%)$ and were extrapolated using SPSS' missing value analysis. This technique imputes missing data via regression. Next, a test on the means of both the high and low blood fear group was conducted to establish whether the two groups indeed differed with respect to blood fearfulness and on disgust propensity and sensitivity.

To determine whether the two disgust scripts elicited a distinct subjective and physiological response pattern, a series of repeated measurement ANOVAs was performed. Two a priori contrasts were specified. In order to determine whether guided imagery of the two disgust types elicited subjective (emotions, symptoms) and physiological responses, the first contrast compared both disgust scripts with the neutral script. To examine whether the different disgust types displayed a distinct reaction pattern, the second contrast compared the subjective and physiological responses during the core disgust script versus the reaction during the A-R disgust script. Further, the group variable was included in all analyses to determine whether high and low blood fearful individuals reacted differently to both disgust types, or to A-R disgust specifically $\neq$.

The analyses were conducted in this order: self-report indices, general physiological arousal and disgust indices (facial EMG of corrugator and disgust-specific levator labii, skin conductance level), core disgust parameter (saliva production), A-R disgust parameters (power in HF band of heart rate variability, T-wave amplitude, and mean arterial pressure).

\section{Results}

\section{Descriptives}

As expected, the high blood fearful group scored significantly higher than the low blood fear group on fear of blood (BIQ, MFS, MBPI), disgust propensity (DS, DQ, DPSS-RP) and disgust

¥¥ First, a one-way ANOVA compared the mean physiological activity on all parameters between the high and low blood fearful participants during the neutral script. Only corrugator activity and saliva production were significantly higher in the high blood fearful group. 
sensitivity (DPSS-RS). Table 1 presents a summary of these findings. Next, a 2 (Scale: DS-Core Disgust, DS-A-R Disgust) x 2 (Group: High, low blood fearful) ANOVA was performed to determine whether high blood fearful individuals displayed elevated disgust propensity specifically for A-R stimuli. Most importantly for the present context, the interaction term Scale $x$ Group $\left(f(1,58)=5.48, p=.02, \eta_{p}^{2}=.09\right)$ and a series of post-hoc paired sample $t$-tests showed that the high blood fearful group scored significantly higher on DS-A-R than on DS-Core $(t(29)=-2.48 ; p=.02)$, whereas the low blood fearful group displayed no differences between the DS-subscales $(t(29)=.85 ; p=.40)$. So, although the high blood fearful participants scored higher on both core and A-R disgust propensity, elevated disgust propensity in this group was most pronounced for A-R disgust.

Table 1.

Mean scores on all questionnaires (MBPI, MFS, BIQ, DS, DQ, and DPSS-R) for the high and low blood fearful groups.

\begin{tabular}{lccccl}
\hline Indices & $\operatorname{HBF}(\mathrm{n}=30)$ & $\mathrm{LBF}(\mathrm{n}=30)$ & Possible range & $t(58)$ & $p$ \\
\hline MBPI total & $63.50(23.11)$ & $8.30(8.67)$ & $0-160$ & -12.25 & $<.01$ \\
MFS total & $74.23(30.30)$ & $21.37(12.14)$ & $0-200$ & -8.87 & $<.01$ \\
BIQ-Fear & $27.83(6.53)$ & $14.70(3.20)$ & $10-50$ & -9.89 & $<.01$ \\
BIQ-Faint & $11.37(2.17)$ & $10.10(.40)$ & $10-30$ & -3.14 & $<.01$ \\
DS total & $19.55(3.97)$ & $14.02(3.87)$ & $0-32$ & -5.46 & $<.01$ \\
Core & $11.73(2.92)$ & $9.23(2.86)$ & $0-18$ & -3.35 & $<.01$ \\
A-R & $12.97(2.31)$ & $8.80(2.92)$ & $0-18$ & -6.13 & $<.01$ \\
DQ total & $114.30(36.78)$ & $142.77(36.54)$ & $24-216$ & 3.01 & $<.01$ \\
DPSS-RP & $23.70(4.40)$ & $17.90(3.14)$ & $16-80$ & -5.87 & $<.01$ \\
DPSS-RS & $19.63(4.94)$ & $13.80(3.21)$ & $16-80$ & -5.43 & $<.01$ \\
\hline Valid N & & & & & 60
\end{tabular}

Abbreviations: $\mathrm{HBF}=$ High blood fearful, $\mathrm{LBF}=$ Low blood fearful, MBPI $=$ Multi-dimensional Blood $/$ Injury Phobia Inventory, MFS = Medical Fear Survey, BIQ = Blood-Injury Phobia Questionnaire, DS = Disgust Scale, A-R = AnimalReminder subscale, DQ = Disgust and Contamination Sensitivity Questionnaire, DPSS-RP $=$ Disgust Propensity and Sensitivity Scale-Revised Propensity, DPSS-RS = Disgust Propensity and Sensitivity Scale-Revised Sensitivity. Note. Standard deviations are described in parentheses.

\section{Differential reactivity to the disgust scripts}

All means and standard deviations of the subjective and physiological parameters can be found in Table 2.

\section{Self-report}

Imaginary ability. A 3 (Script: Neutral, A-R disgust, Core disgust) x 2 (Group: High, low blood fearful) ANOVA was performed for the ability to imagine the scripts. Interaction Script $x$ Group was not significant $\left.(f 2,57)=.41, p=.66, \eta_{p}^{2}=.01\right)$. Next, according to a significant effect for Script, some scripts could be better imagined than others $\left(f(2,57)=3.76, p=.03, \eta_{p}^{2}=.12\right)$. Post-hoc contrasts revealed that overall, participants could imagine the A-R script less well than the neutral script $\left(F(1,58)=7.65, p<.01, \eta_{p}^{2}=.12\right)$. Yet, although the core script was 


\section{CHAPTER 8}

imagined equally well to the neutral script $\left(f(1,58)=1.37, p=.25, \eta_{p}^{2}=.03\right)$, no differences were observed between the imaginability of the core and A-R script $(f(1,58)=2.04, p=.16$, $\left.\eta_{p}^{2}=.03\right)$. Further, high and low fearful participants did not differ in their ability to imagine either script $\left(\neg(1,58)=.21, p=.65, \eta_{p}^{2}<.01\right)$.

Table 2.

Means (standard deviations) of the self-report and physiological parameters for all scripts as a function of blood fear.

\begin{tabular}{|c|c|c|c|c|c|c|}
\hline & \multicolumn{2}{|l|}{ Neutral } & \multicolumn{2}{|l|}{ Core Disgust } & \multicolumn{2}{|l|}{ A-R Disgust } \\
\hline & $\overline{\mathrm{LBF}}$ & HBF & LBF & $\mathrm{HBF}$ & LBF & $\mathrm{HBF}$ \\
\hline \multicolumn{7}{|l|}{ Self-report } \\
\hline Imagine & $74.10(20.06)$ & $78.30(16.64)$ & $71.43(24.07)$ & $73.77(16.74)$ & $68.57(21.81)$ & 67.33 (21.41) \\
\hline Disgust & 1.43 (2.99) & $6.93(14.02)$ & $42.20(26.88)$ & $63.63(22.71)$ & $31.93(29.42)$ & $57.47(20.71)$ \\
\hline Fear & $2.10(4.20)$ & $10.97(12.33)$ & $8.00(12.44)$ & $23.53(19.45)$ & $9.13(12.00)$ & $27.50(18.50)$ \\
\hline Vomit & $.97(1.87)$ & $5.50(14.16)$ & $8.53(16.17)$ & $40.13(29.45)$ & $2.67(7.07)$ & $24.90(28.01)$ \\
\hline Dizzy & $1.67(2.94)$ & $2.77(5.48)$ & $2.97(7.32)$ & $9.40(11.50)$ & $1.77(3.43)$ & 7.97 (12.01) \\
\hline \multicolumn{7}{|c|}{ General parameters } \\
\hline $\operatorname{Lev}(\mu \mathrm{V})$ & $9.26(3.40)$ & $10.73(4.83)$ & $10.83(6.62)$ & $13.42(4.98)$ & $10.92(5.90)$ & $14.33(6.38)$ \\
\hline Corr $(\mu \mathrm{V})$ & $9.11(3.91)$ & 13.39 (9.89) & $9.28(4.05)$ & $15.73(9.04)$ & $9.20(3.01)$ & $15.53(9.04)$ \\
\hline $\mathrm{SCL}(\mu \mathrm{S})$ & $1.65(.92)$ & $1.45(.94)$ & $1.65(.85)$ & $1.57(1.06)$ & $1.71(.83)$ & $1.61(1.10)$ \\
\hline \multicolumn{7}{|c|}{ Digestive component } \\
\hline Saliva (g) & $1.71(1.20)$ & $2.67(1.84)$ & $1.69(1.22)$ & $3.02(2.11)$ & $1.73(1.15)$ & $3.23(2.41)$ \\
\hline \multicolumn{7}{|c|}{ Cardiac components } \\
\hline $\operatorname{HRV}(\mathrm{nu})$ & $71.03(31.60)$ & $64.55(29.62)$ & $65.89(30.63)$ & $52.00(20.02)$ & $50.71(114.61)$ & $62.52(42.17)$ \\
\hline \multirow[t]{2}{*}{ TWA $(\mu \mathrm{V})$} & 333.32 & 316.00 & 345.73 & 324.70 & 343.60 & 324.89 \\
\hline & $(130.09)$ & $(129.06)$ & $(136.70)$ & $(130.85)$ & $(137.71)$ & $(130.84)$ \\
\hline $\mathrm{MAP}(\mathrm{mmHg})$ & $92.39(11.20)$ & $92.51(12.46)$ & $91.86(10.73)$ & $94.45(12.45)$ & $92.82(9.34)$ & 93.75 (12.58) \\
\hline Valid N & & & & & & 60 \\
\hline
\end{tabular}

Abbreviations: HRV = Heart Rate Variability in High Frequency Band, Lev = Levator, Corr = Corrugator, $\mathrm{SCL}=\mathrm{Skin}$ Conductance Level, TWA = T-wave Amplitude, MAP = Mean Arterial Blood Pressure, LBF = Low Blood Fearful Group, $\mathrm{HBF}=$ High Blood Fearful Group; $\mathrm{g}=$ grams; nu = normalized units.

Standard deviations are presented in parentheses

Differential emotional reactivity. A 2 (Emotion: Disgust, Fear) x 3 (Script: Neutral, A-R disgust, Core disgust) $\times 2$ (Group: High, low blood fearful) ANOVA revealed significant effects for Script $\left(f(2,57)=99.70, p<.01, \eta_{p}^{2}=.78\right)$, Script $x$ Emotion $\left(f(2,57)=70.81, p<.01, \eta_{p}^{2}=.71\right)$ and Script $x$ Group $\left(F(2,57)=5.39, p=.01, \eta_{p}^{2}=.16\right)$. The third order interaction Script $\mathrm{x}$ Emotion $x$ Group was not significant $\left(F(2,57)=1.54, p=.22, \eta_{p}^{2}=.05\right)$. Planned contrasts for the Script effect showed that both disgust scripts evoked stronger emotions than the neutral script $\left(F(1,58)=192.70, p<.01, \eta_{\mathrm{p}}^{2}<.77\right)$, but that there was no difference between the core and A-R disgust script $\left(\Re(1,58)=1.98, p=.17, \eta_{p}^{2}=.03\right)$. Furthermore, the significant contrast "disgust scripts versus the neutral script" for the Script $\mathrm{x}$ Emotion effect $(f(1,58)=$ $139.16, p<.01, \eta_{p}^{2}=.71$ ) revealed that specifically for the disgust scripts, the experience of disgust is stronger than that of fear. The contrast "A-R vs. core disgust" was significant $(\Re(1,58)$ $=14.26, p<.01, \eta_{p}^{2}=.20$ ), showing that the core disgust script evoked stronger disgust than the A-R script. Finally, the contrasts for the Script $x$ Group interaction indicated that high blood fearfuls experienced higher levels of fear and disgust than low blood fearfuls during both 
disgust scripts compared to the neutral script $\left.(F 1,58)=10.77, p<.01, \eta_{p}^{2}=.16\right)$. This effect was similar for both types of disgust scripts $\left.(\digamma 1,58)=.74, p=.39, \eta_{p}^{2}=.01\right)$.

Vasovagal vs. digestive symptoms. A 2 (Response: Dizziness, Tendency to vomit) $\times 3$ (Script: Neutral, A-R disgust, Core disgust) $\times 2$ (Group: High, low blood fearful) ANOVA revealed significant Script $\left(F(2,57)=21.77, p<.01, \eta_{p}^{2}=.43\right)$, Script $\times$ Response $(F(2,57)=18.23, p<$ $\left..01, \eta_{p}^{2}=.39\right)$ and Script $\times$ Group $\left(F(2,57)=11.08, p<.01, \eta_{p}^{2}=.32\right)$ effects. The third order interaction Script $x$ Response $x$ Group was also significant $\left.(F 2,57)=7.48, p<.01, \eta_{p}^{2}=.21\right)$. Planned contrasts for the Script effect showed that both disgust scripts evoked stronger symptoms than the neutral script $\left.(F 1,58)=43.01, p<.01, \eta_{p}^{2}<.42\right)$ and core disgust script evoked stronger symptoms than the A-R disgust script $\left(F(1,58)=12.91, p<.01, \eta_{p}^{2}=.18\right)$. Both planned contrasts for the Script $x$ Response effect were significant, demonstrating that the tendency to vomit was stronger than the experience of dizziness during the disgust scripts $\left.(F 1,58)=28.49, p<.01, \eta_{p}^{2}=.33\right)$ and that this difference was more prominent during the core disgust script than the A-R disgust script $(f 1,58)=11.85, p<.01, \eta_{p}^{2}=.17$; see Table 2). The first planned contrast (disgust vs. neutral) for the Script $x$ Group interaction and the Script $x$ Response $x$ Group interaction indicated that high blood fearfuls generally experienced symptoms more strongly than the low blood fearful participants during the disgust scripts $\left.(F 1,58)=22.37, p<.01, \eta_{p}^{2}=.28\right)$, yet this was most apparent for the tendency to vomit during the disgust scripts $\left(f(2,57)=13.39, p<.01, \eta_{p}^{2}=.19\right)$. The second planned contrast (core disgust vs. A-R disgust) did not reach significance for the Script $x$ Group interaction $\left.(F 1,58)=2.11, p=.15, \eta_{p}^{2}=.04\right)$ nor for the Script $\times$ Response $\times$ Group interaction term $\left(F(2,57)=2.90, p=.09, \eta_{p}^{2}=.05\right)$. Thus, high blood fearfuls experienced more dizziness and especially a stronger tendency to vomit than low blood fearfuls independent of the type of disgust elicitor.

\section{General disgust and arousal parameters}

\section{Levator labii}

A 3 (Script: Neutral, A-R disgust, Core disgust) $x 2$ (Group: high, low blood fearful) ANOVA was performed for the disgust-specific $m$. levator. The interaction term Script $x$ Group did not reach significance $\left.(F 1,55)=.61, p=.55, \eta_{p}^{2}=.02\right)$. Yet, the main effect Script was significant $\left.(F 2,55)=4.87, p=.01, \eta_{p}^{2}=.15\right)$. The first contrast for Script showed that levator activity increased during both disgust scripts compared to the neutral script $(f 1,56)=9.80, p<.01$, $\eta_{p}^{2}=.15$ ). The second planned contrast showed that levator activity did not differ between the A-R and core disgust script $\left.(\digamma 1,56)=.73, p=.40, \eta_{p}^{2}=.01\right)$.

\section{Corrugator}

A 3 (Script: Neutral, A-R disgust, Core disgust) $\times 2$ (Group: high or low blood fearful) ANOVA was performed for the $\mathrm{m}$. corrugator. The interaction term Script $\times$ Group was not significant $\left(F(2,55)=1.96, p=.15, \eta_{p}^{2}=.07\right)$ and neither was the main effect for Script $(F(2,55)=2.40$, 


\section{CHAPTER 8}

$p=.10, \eta_{p}^{2}=.08$ ), so the two disgust scripts did not evoke increases in corrugator activity compared to the neutral script.

\section{Skin conductance level}

A 3 (Script: Neutral, A-R disgust, Core disgust) $\times 2$ (Group: high or low blood fearful) ANOVA was performed for skin conductance level. Interaction term Script $x$ Group was not significant $\left.(F 2,56)=1.25, p=.29, \eta_{p}^{2}=.04\right)$, so both high and low blood fearful individuals displayed similar patterns of skin conductance during the various scripts. The main effect Script was significant $\left.(F 1,58)=3.91, p=.03, \eta_{p}^{2}=.12\right)$. Planned contrasts showed that compared to the neutral script, the two disgust scripts induced significant increases in skin conductance level $\left(F(1,57)=5.56, p=.02, \eta_{p}^{2}=.09\right)$. The second contrast showed that skin conductance level generally did not differ between the A-R and core disgust script $\left(f(1,57)=2.67, p=.11, \eta_{p}^{2}=\right.$ .05).

\section{Core disgust parameter}

\section{Salivary excretion}

A 3 (Script: Neutral, A-R disgust, Core disgust) x 2 (Blood Fear: high or low) ANOVA was performed on salivary excretion in grams. Both the interaction term Script $\times$ Group $(F(2,57)=$ 2.47, $\left.p=.09, \eta_{p}^{2}=.08\right)$ and the main effect Script $\left(F(2,57)=2.54, p=.09, \eta_{p}{ }^{2}=.08\right)$ approached significance. Contrasts for main effect Script showed that overall, participants produced significantly more saliva during the two disgust scripts compared to the neutral script $\left.(F 1,58)=4.91, p=.03, \eta_{p}^{2}=.08\right)$. Yet, the salivary excretion did not differ significantly between A-R script and the core disgust script $\left.(F 1,58)=1.41, p=.24, \eta_{p}^{2}=.02\right)$. For the interaction term Script $x$ Group, the salivary excretion was greater in the high blood fearful group compared to the low fearful group during the two disgust scripts versus the neutral script $\left(F(2,57)=5.02, p=.03, \eta_{p}^{2}=.08\right)$. The second contrast showed that the difference in saliva production during $A-R$ disgust versus the core disgust script did not differ between the two groups $\left(f(1,58)=.59, p=.45, \eta_{p}^{2}=.01\right)$.

\section{$A-R$ parameters}

\section{Heart rate variability}

A 3 (Scripts: Neutral, A-R disgust, Core disgust) $\times 2$ (Group: high or low blood fearful) ANOVA was performed for power in normalized units in the high frequency band. The interaction term Script $x$ Group was not significant $\left.(F 2,54)=1.86, p=.17, \eta_{p}^{2}=.06\right)$ while main effect Script did approach significance $\left.(F 2,54)=2.51, p=.09, \eta_{p}^{2}=.09\right)$. Contrasts for the main effect Script demonstrated that heart rate variability did not differ during the two disgust scripts 
compared to the neutral script $\left(f(1,55)=1.46, p=.23, \eta_{p}^{2}=.06\right)$ nor between the A-R script and the core disgust script $\left(f(1,55)=.04, p=.83, \eta_{p}^{2}<.01\right)$.

\section{T-wave amplitude}

For the T-wave amplitude, a 3 (Script: Neutral, A-R disgust, Core disgust) x 2 (Group: high or low blood fearful) ANOVA was performed. The interaction term Script $x$ Group was not significant $\left(F(1,55)=.26, p=.78, \eta_{p}^{2}=.01\right)$. The main effect Script was significant $(f(1,55)=$ 9.10, $\left.p<.01, \eta_{p}^{2}=.25\right)$. Next, planned contrasts for the main effect Script showed that generally, participants responded to the two disgust scripts with an increase in T-wave amplitude compared to the neutral script $\left(f(1,56)=18.47, p<.01, \eta_{\mathrm{p}}^{2}=.25\right)$ but no significant differences were observed in T-wave amplitude during the A-R script versus the core disgust script $\left(F(1,56)=.18, p=.68, \eta_{p}^{2}<.01\right)$.

\section{Mean arterial pressure}

A 3 (Script: Neutral, A-R disgust, Core disgust) x 2 (Group: high or low blood fearful) ANOVA was performed for the mean arterial pressure. The interaction term Script $x$ Group was significant $\left(F(2,53)=3.31, p=.04, \eta_{p}^{2}=.11\right)$. The main effect Script $(F(2,53)=1.29, p=.28$, $\left.\eta_{\mathrm{p}}^{2}=.05\right)$ was not significant. For the interaction term Script $x$ Group, planned contrasts revealed that a border-significant increase was observed in mean arterial pressure during both disgust scripts, but only in high blood fearful participants $\left(f(1,54)=2.93, p=.09, \eta_{p}^{2}=.05\right)$. Further, the second contrast revealed a border-significant effect $\left(f(1,54)=3.19, p=.08, \eta_{p}^{2}=\right.$ $.06)$. Thus, mean arterial pressure tended to be lower during the core disgust script compared to the A-R disgust script in low blood fearful participants, while the reverse appeared true for the high blood fearful group.

\section{Discussion}

The present study represents the first attempt to test the idea of differential responsivity of cardiovascular and digestive components of the ANS during core and A-R disgust. The main findings were as follows: i) guided imagery of disgust elicited a physiological response pattern characterized by increases in saliva production, in T-wave amplitude and in EMG activity of the $\mathrm{m}$. levator labii, ii) A-R and core disgust did not evoke a distinct physiological response pattern in this respect, yet iii) overall, the core disgust script did evoke stronger subjective experiences than the A-R script (i.e., stronger feelings of disgust, fear, dizziness and tendency to vomit), and finally iv) disgust-induced subjective responsivity was most pronounced in high blood fearful individuals, although this was not mimicked by stronger physiological reactivity.

In line with earlier research (e.g., Vrana, 1993, de Jong, Peters, \& Vanderhallen, 2002), guided imagery successfully elicited self-reported feelings of disgust and induced disgustspecific activity in facial EMG (i.e., increased activity of the m. levator labii). Most relevant for 


\section{CHAPTER 8}

the present context, the elicited feelings and expressions of disgust during the core and A-R disgust scripts were accompanied by changes in activity in the digestive as well as the cardiac components of the ANS. For the digestive component, increases were observed in saliva production, which most likely indicates an increase in parasympathetic activity (Proctor \& Carpenter, 2007). For the cardiac components, increases were observed in T-wave amplitude which generally can be taken to indicate sympathetic withdrawal (Kline et al., 1998). There was no evidence for increased parasympathetic activity of the cardiac component of the ANS; participants' heart rate variability (represented by power in the HF band) remained unaffected by the disgust induction.

The disgust-induced physiological changes were paralleled by a rise in subjective indicators of digestive and cardiac reactivity (i.e., vomit tendencies and feelings of dizziness, respectively). These subjective symptoms were significantly stronger during the core disgust than during the A-R disgust script. This was not the case for the physiological indices. By and large, the physiological response pattern was very similar for core and A-R disgust. Previous research indicated that core and A-R disgust can be reliably distinguished from each other on a subjective level (Olatunji et al., 2007). So it appears that although the content of elicitors in A-R disgust may have gradually evolved away from core disgust elicitors (Rozin \& Fallon, 1987; Rozin et al., 1999), the autonomic response program for both disgust types remained the same: a defensive response program shielding the organism from contamination by pathogens. This may also explain why mainly the digestive component of the PNS became activated in the present disgust imagery procedures, as vomiting is responsible for the expulsion of potentially hazardous pathogens from the body (e.g., by vomiting) (Guyton \& Hall, 2006).

For both types of disgust-imagery, the pattern of disgust-induced ANS reactivity was largely similar for high and low blood fearful individuals, and no evidence emerged to indicate that high blood fearful individuals showed an increase in parasympathetic cardiac activity during (A-R) disgust and/or decrease in mean arterial pressure. This contradicts the idea that specifically people who respond with a greater parasympathetic activity (and subsequent drop in blood pressure) during confrontation with (A-R) disgust stimuli are at risk for developing blood phobia (e.g., as these people may be more prone to emotional fainting; de Jong \& Merckelbach, 1998). In addition, these results are in apparent contrast with the earlier findings that blood fearful individuals did demonstrate parasympathetic reactivity in cardiac measures during exposure to blood-related stimuli (e.g., surgery films), such as a decrease in blood pressure (Öst, Sterner, Lindahl, 1984; Rohrmann \& Hopp, 2008 ; Vögele, Coles, Wardle, \& Steptoe, 2003) or an increase in heart rate followed by an abrupt drop (Palomba et al., 2000; Sarlo et al., 2002). However, because all of these earlier studies used blood-related material, it may well be that a diphasic response in cardiac components may be specific to blood rather than for (A-R) disgust stimuli in general.

Although high and low blood fearful participants showed a very similar physiological response pattern, marked differences were evident at the subjective level. Irrespective of the type of disgust imagery, high blood fearful participants generally experienced stronger increases in subjective emotional reactivity (both disgust and fear), and a relatively strong experienced tendency to vomit and feelings of dizziness in response to the disgust scripts. The differences in 
subjective symptoms (dizziness and vomit tendencies) between the high and low blood fearful participants were most pronounced for the core rather than the A-R script. This is particularly noteworthy since this contradicts earlier postulations that a propensity for A-R stimuli may be associated with specific symptomatology in blood phobia (i.e., fainting; de Jong \& Merckelbach, 1998).

Sustaining the alleged role of (subjective) disgust in blood phobia and consistent with the present finding that high blood fearful individuals experienced relatively strong disgust during disgust imagery, they also reported a stronger habitual tendency to respond with disgust in response to a variety of potential disgust elicitors than low fearful individuals. In line with earlier research (de Jong \& Merckelbach, 1998), elevated disgust propensity was most pronounced for A-R disgust. In apparent contrast with this, the enhanced experience of disgust in high blood fearful individuals during the present imagery procedure was similar for A-R and core disgust imagery. Interestingly, blood fearful participants not only displayed elevated levels of disgust propensity but also heightened disgust sensitivity (the tendency to evaluate the experience of disgust more negatively). These findings are in line with recent indications that disgust sensitivity could play a pivotal role in the etiology of blood fear (e.g., van Overveld et al., 2006). As high blood fearful individuals generally experience disgust in response to blood (Tolin et al., 1997), it may well be that an extreme sensitivity for experiencing disgust induces an avoidance pattern for the physical correlates of disgust, rather than the confrontation with blood stimuli per se.

Some comments are in order regarding the methodological aspects of the current experiment. First, it could be that the imagination procedure itself influenced the results of the response patterns. For example, the use of imagination procedures renders it difficult to establish whether participants intentionally decide to imagine certain scripts less vividly to avoid possible aversive consequences (i.e., fainting). From that perspective, it would be interesting to compare imagery with other types of elicitors (e.g., using film clips or behavioral approach experiments) to examine if these would yield different results. In one of the few studies comparing guided imagery of disgust with an intense (core) disgust film, both methods successfully induced disgust as reflected by self-report and facial EMG, yet, only the pattern following the film clip differentiated between high and low (spider) fearful groups (de Jong, Peters, \& Vanderhallen, 2002).

Second, the present design only included neutral and disgusting materials. Therefore, it remains to be established whether the physiological reactivity in response to the disgust scripts are specific to disgust or could be associated with other emotions as well. Thus, to determine the specificity of the various parameters to disgust, future research may consider including scripts on both negative (i.e., fear) and positive emotions (i.e., happiness). In addition, although the A-R script did evoke disgust and did not differ in imaginability compared to the other scripts, it may be argued that the A-R script was relatively bizarre. Consequently, the A-R disgust script may not have been as effective as the other script types in inducing its alleged concomitant physiological response pattern.

Third, the order of presentation of the scripts was relatively fixed in the sense that the neutral script was always presented prior to the disgust scripts in an attempt to prevent 


\section{CHAPTER 8}

undesirable carry-over effects from the disgust script on the neutral condition. Thus, any differences between the neutral and the two disgust scripts may be explained in terms of habituation or cross-over effects. At least some of the present parameters are known for their short-lived and event-related reactivity (i.e., levator labii) so these may be less sensitive to cross-over and habituation effects. It might have been more problematic for other measures (i.e., SCL and power in the HF band). Yet, no changes were noted in HRV during the disgust scripts compared to the neutral script, whereas SCL increased rather than decreased during the disgust scripts. This is in apparent contrast with a habituation explanation, and in line with recent work that found similar elevations in SCL during disgusting film clips (Rohrmann \& Hopp, 2008). Thus, it appeared that habituation and/or the order of presentation of the scripts did not play a major role in the present pattern of findings.

In conclusion, the present findings indicated that inducing disgust coincided with an increase in parasympathetic activity (i.e., saliva production), with a reduction in sympathetic activity (i.e., increases in T-wave amplitude) and with increases in facial EMG of the m. levator. In conflict with the proposed role of disgust-induced autonomic activity in blood phobia (Page, 1994), the disgust-induced physiological reactivity was not particularly pronounced in high blood fearful participants. Germane to this, physiological activation was shown to be relatively independent of the type of disgust elicitor (core versus A-R). We therefore propose that the physiological reaction pattern of core disgust functions as a hard-wired defensive mechanism to protect the individual against contamination with possible pathogens, and provides a basis for all disgust reactions. 


\section{Appendix}

\section{Neutral}

You are about to look up a friend who has moved to another city. You are standing at the platform of the station, waiting for the right train. Concentrate on what you see on the station. Pay attention to all the sounds which you hear. A man comes by with strong aftershave. Concentrate yourself on the fragrance. Gradually it becomes more busy at the platform. Concentrate yourself on what you observe when the train approaches the platform. You are standing at the door with two other people. You get in the train. You are searching for a seat. Pay attention to what you see. You have found a seat. You lay down your stuff and will sit down. Concentrate yourself on your surroundings. Look around the carriage to see what kind of people sit there. How do they look? What are they doing? Concentrate on what you hear. You look outside. What do you see if you look outside? The conductor comes in and asks for the tickets. What does he look like? What happens when he enters the carriage? You look in your pockets to grab your ticket. You show your ticket to the conductor. On your watch you notice that in some minutes, you will arrive at the station of your destination. The train decreases in speed. Concentrate yourself on what you feel in your body when the train slows down. You have arrived at your end destination. Consider what it looks like when the train drives in the station. Concentrate on what you hear. Concentrate yourself on what happens in the carriage. You take your case and get up. You are walking through the station in search of the exit. What does the station look like? Concentrate yourself on what you hear. You follow the stream of people towards the exit. Concentrate yourself on how you feel. From your friend you obtained a route description. Following the route description, you walk through the streets of the city. What do you see around you? Which people are walking there? Concentrate on the sounds of the city. You reach the street where you must be. Halfway the street you discover the house number where you must be. You ring and your friend opens the door.

\section{Oral disgust}

You work as an assistant in home care. You open the door at an old man. You hear from the sounds coming from the chamber that something is wrong. Concentrate yourself on what you hear. You open the door. The man appears sick and has vomited. Apparently he was not able to reach the bathroom in time. Concentrate yourself on what you smell. Which fragrances can you distinguish? Concentrate yourself on what you see. What does the man look like? How does he sit? You walk up to the man. Concentrate yourself on the fragrance. Pay attention to the details which you see now. What does his shirt look like? See how fresh vomit mixes with the dried-up remains of vomit from an hour ago. Concentrate on the face of the man, what does this look like? Observe the lumps of vomit that drip down along his chin. The man vomits again. Concentrate yourself on his body. What do you see happening? Concentrate yourself on his face. Concentrate on the sounds of vomiting. Take in the sour fragrance. What does the goo look like that the man spits out? You are gagging. Concentrate yourself on what you feel happing in your stomach, throat and mouth. Incorporate the sour taste in your mouth. Some splashes of vomit spatter on your hands and your face. How do you feel? While you help the 


\section{CHAPTER 8}

man out of his clothing, you notice that he is defecating himself. Concentrate yourself on what you see, what you smell and what you hear. You are helping the man. Concentrate on your physical feelings. You are washing the man. What does this look like? Which operations do you have to perform? What does this do to you?

\section{Animal-reminder disgust}

You are sitting at a square. Directly across you sits a somewhat dirty looking man. Look closely at the man. Pay attention to what you see. You look at to the face of the man and you see that his right eye is not real. Concentrate yourself on what you feel. You look more closely and notice that his right eye is made of glass. What does it look like? Concentrate on what you see and how you feel. You look at the man. You look at his face with his glass eye. Pay attention to what you see. Then, the man brings a spoon to his glass eye. He puts the spoon behind his eye and hops the eye from its socket. Concentrate yourself on what you see and what you hear. Concentrate on your feelings. Pay attention to your pulse and your breathing. You look at the empty cavity where the glass eye used to be. Concentrate yourself on the empty eye socket. Pay attention to what the empty cavity looks like. Concentrate yourself on what you see and how you feel. You look at to the spoon bearing the glass eye. Concentrate yourself on the spoon with the glass eye. You look at the man and you see that he is now taking out a cow's eye. Concentrate on how the cow's eye looks like. Pay attention to what you feel. You see the cow's eye lying on the hand of the man. The man studies the eye from all sides. Pay attention to what you see. Concentrate on what you feel. After the man has inspected the cow's eye thoroughly, you see how he brings the eye to his face. Concentrate yourself on what you see and how you feel. The man takes the cow's eye in the direction of the empty eye socket. Then, he puts the cow's eye in his own empty eye socket. What does this do to you? Pay attention to what you see. Concentrate on what you feel. Pay attention to your pulse and your breathing. You look closely at the man again and look at the cow's eye that is now in the face of the man. Concentrate yourself on the cow's eye in the face of the man. Pay attention to what you see. At that moment, the man looks at to you. He is looking straight in your eyes. Concentrate yourself on what you see and how you feel. 


\section{Chapter 9}

\section{Differential UCS expectancy bias in spider fearful individuals: evidence towards an association between spiders and disgust-relevant outcomes}




\section{CHAPTER 9}

\section{Abstract $\$$ §§}

Recently, differential UCS expectancies were found for high- and low predatory fear-relevant animals (Davey et al., 2003). The present study extends these findings to spider phobia. In a hypothetical experiment, high $(n=27)$ and low $(n=28)$ spider fearful individuals estimated the probability that slides of spiders, maggots, pit bull terriers, or rabbits would be followed by a sip of nauseating juice, a shock, or nothing. Maggots were selectively associated with the disgusting juice, pit bull terriers with the harm-related shock, and rabbits with nothing. Spiders were associated with both aversive UCSs, but significantly stronger in the high fear group. Additionally, an expectancy bias towards disgust-relevant consequences was the single best predictor of spider fear. These findings imply that in accordance with the disease-avoidance model, expectations of disgust-relevant consequences are involved in spider phobia.

\footnotetext{
$\S \S$ Published as: van Overveld, M., de Jong, P. J., \& Peters, M. L. (2006). Differential UCS-expectancy bias in spider fearful individuals: Evidence toward an association between spiders and disgust-relevant outcomes. Journal of Behavior Therapy and Experimental Psychiatry, 37, 60-72.
} 
There is considerable evidence that phobic individuals have a tendency to over-associate encounters with phobic stimuli with the occurrence of aversive consequences (e.g., Davey, 1992a; Tomarken, Mineka \& Cook, 1989). Thus, upon confrontation with their phobic objects, fearful individuals expect an aversive consequence to occur. Accordingly, it has been shown under laboratory conditions that phobic individuals display inflated expectancies that aversive consequences, for example electrical shocks, will follow fear-relevant stimuli. This has been found in different kinds of anxiety disorders, such as snake phobia (McNally \& Heatherton, 1993), flight phobia (Pauli, Wiedemann, Montoya, 1998), and panic disorder (Wiedemann, Pauli \& Dengler, 2001). Such bias logically acts in a way that contributes to the continuation and heightening of fear.

Expectancy bias seems also involved in spider phobia. In a 'thought' experiment procedure, spider phobics reported higher expectations of an aversive outcome (electrical shock) to follow the occurrence of spider stimuli than non-phobics (Cavanagh \& Davey, 2000; Öhman, Dimberg \& Öst, 1985). Moreover, when actually exposed to a random combination of slides (snakes, spiders, electrical outlets, flowers) and outcomes (shock, tone or nothing), specifically high spider fearful participants overestimated the covariation between spiders and the occurrence of aversive outcomes (shock) (Kennedy, Rapee \& Mazurski, 1997).

The expectation that aversive, harm-related consequences will follow upon the presentation of spider slides is consistent with the predator-defense model. This model postulates that a fear of small animals is derived from defensive strategies, which have developed as a result of the threat of predators (Öhman et al., 1985). Spiders and snakes would thus be feared due to their harmful, predatory properties. Yet, there is accumulating evidence that not only threat-related beliefs contribute to a fear of spiders, but also disgust-related preoccupation with the spider (Sawchuk, Lohr, Tolin, Lee \& Kleinknecht, 2000; Tolin et al., 1997). Accordingly, when viewing spider slides, spider phobics not only report feelings of fear, but also feelings of disgust (Sawchuk et al., 2002; Tolin et al., 1997). In addition, spider phobics report that spiders are not only their most feared objects, they are also the ones they find most disgusting (Thorpe \& Salkovskis, 1998). Also, when confronted with spiders, highly spider fearful people respond with greater disgust-specific facial EMG activity (activity of the $\mathrm{m}$. levator labii) than explicitly nonfearful people (de Jong, Peters, \& Vanderhallen, 2002).

Underlining the relative importance of disgust-related convictions, recent research has shown that fear of being contaminated with a disgusting object (a spider) is a better predictor of spider fear than the fear of physical harm (de Jong \& Muris, 2002). All of these findings are consistent with the disease-avoidance model, which states that a fear of harmless, small animals is not the result of expecting harm from a confrontation with these animals, but rather originates from a fear of contamination with dirt or disease (Matchett \& Davey, 1991).

So far, research on expectancy bias has almost exclusively focused on expectations of harmrelated consequences (e.g., an electrical shock) while according to the disease-avoidance model, disgust-related outcomes might provide a more likely explanation of spider phobia. Germane to this, it has been shown that outcome expectancies vary depending on the type of CS that is presented (Davey, Cavanagh, \& Lamb, 2003). That is, in a 'thought' experiment, participants were asked to rate the probability that slides of different kinds of animals would be 


\section{CHAPTER 9}

followed by one of three consequences, namely by an electrical shock, a sip of a disgusting drink or no response at all. Three types of animals were used in this experiment: low predatory fear-relevant animals (spider, maggot, cockroach, slug), high predatory fear-relevant animals (tiger, wolf, shark, snake) and safe fear-irrelevant animals (rabbit, kitten, sheep, chicken). Results indicated that the fear-relevant high predatory animals were selectively associated with the harm-related consequence (shock) and fear-relevant but low predatory animals with the disgust consequence (nauseating drink). Neutral animals were associated with neither shock nor drink (Davey et al., 2003). Most important for the present context is that the low-predatory fear-relevant animals in that study were associated with a disgusting outcome, and not with a harm-related consequence (shock). The present study has elaborated on these findings on the existence of differential UCS-expectancies for various animal types, and extended them to spider phobia.

Therefore, the major goal of the present study was to examine the relative importance of disgust and harm-related expectancies in spider fear. As mentioned above, spiders may be feared due to relatively strong harm-related outcome expectancies (predator-defense model) or relatively inflated UCS disgust representations (as would be predicted by the disease-avoidance conceptualization of spider phobia) or by both. To investigate these issues, a group of high spider fearful individuals and a group of low spider fearful individuals were asked to participate in a hypothetical aversive conditioning experiment. They had to estimate the probability that pictures of spiders, maggots, pit bull terriers or rabbits would be followed by one of three outcomes: a) the insertion of a sip of nauseating juice in the mouth via a catheter, b) the administration of a painful electrical shock, or c) nothing at all.

In conclusion, the present study was designed to test: (1) the robustness of the earlier finding of Davey et al. (2003) that differential UCS-expectancies can be found in individuals, independent of prior fear; (2) the prediction that, in line with previous research (de Jong \& Muris, 2002; Tolin et al., 1997), spiders will not only be associated with harm- but also with disgust-related consequences; (3) the hypothesis that, in accordance with previous research (de Jong \& Muris, 2002), high spider fearful participants will associate spiders more strongly with both aversive consequences, yet, disgust-related convictions will differentiate best between high and low spider fearful groups. In other words, high spider fearful people will be characterized by a stronger disgust-related preoccupation with spiders compared to low spider fearful people.

\section{Methods}

\section{Participants.}

Participants were selected from a large sample of Dutch students at the Faculties of Health Science, Medicine, and Psychology at the University of Maastricht $(N=359)$. They were selected on the basis of their spider fear rating on a Visual Analog Scale ranging from 0 (no fear at all) to 100 (extremely fearful). The 35 lowest scoring individuals (VAS < 11) were invited for 
the final screening, as well as the 35 persons with the highest scores (VAS > 77). The final decision to include participants in either the low spider fear group or the high spider fear group was made on the basis of their scores on the Fear of Spiders Questionnaire (see below). A total of 55 persons agreed to participate, of whom 27 were assigned to the low fear group, and 28 to the high fear group. Since the prevalence of spider phobia is much higher in women than in men (Davey, 1997), only women were selected to participate. The mean age was 19.1 years $(S D=1.5)$ in the low fear group and 18.7 years $(S D=0.8)$.

\section{Materials}

Fear of Spiders Questionnaire (FSQ; Szymanski \& $O^{\prime}$ Donohue, 1995). The FSQ is a questionnaire measuring fear of spiders. Participants rate 18 items on an 8-point Likert-type scale, ranging from 0 to 7 (scoring range: $0-126$ ). Items assess how strongly people tend to avoid spiders, and how anxious they become when encountering a spider (e.g. "If I came across a spider now, I would get help from someone else to remove it"). The FSQ has good psychometric properties. It is known to have a high internal consistency $(a=0.92)$ (Szymanski \& O' Donohue, 1995) as well as high test-retest reliability (0.91) (Muris \& Merckelbach, 1996). The FSQ was used to make the final decision to assign a participant to either the high spider fear group (FSQ $>30$ ) or the low spider fear group (FSQ $<30$ ). There was an average score of 5.48 on the FSQ $(S D=7.28)$ in the low spider fear group, and of $65.54(S D=19.13)$ in the high spider fear group. The mean of the high spider fear group is comparable with previous studies using analogue, non-clinical samples $(M=62.3, S D=21.6$; de Jong, Peters, \& Vanderhallen, 2002; $M=61.7, S D=25.6$; Woody \& Tolin; 2002), but are clearly lower than studies using clinical samples $(M=89.1, S D=19.6$; Muris \& Merckelbach, 1996).

\section{UCS-expectancies}

Participants were presented with a verbal description of the experiment, after which they filled in the questionnaire. The instruction was as follows:

\section{Thought experiment}

This brief questionnaire studies how people perceive experimental research. This will be done by studying beliefs about certain animals.

\section{Description of the experiment}

You are about to participate in a thought experiment. This means that we will ask you to imagine as vividly as possible the following situation. You are invited here to participate in an experimental study at the laboratory. You will be asked to sit down in a comfortable chair, and view a series of slides, projected on the large screen in the lab. Two electrodes will be placed on your upper arm and before the experiment starts a level of electrical shock will be selected in consultation with you. This will be done so the level is certainly unpleasant, yet not painful. During the experiment you will receive shocks at certain moments. Also, a catheter will be 


\section{CHAPTER 9}

inserted in your mouth, and taped to your cheek, so at certain moments a fluid can be injected. This fluid will taste very bitter, and is quite nauseating. The fluid is, however, just like the shock, unpleasant yet harmless and without side effects.

During the experiments you will be shown a series of slides of four types of animals: pit bull terriers, spiders, maggots and rabbits (at this point, some exemplary slides were shown of a growling pit bull terrier, a spider, maggots and a white rabbit; see also the Appendix). Each slide will be shown for exactly six seconds and will immediately be followed by one of these three consequences: either you will receive a short but unpleasant electrical shock, or a shot of the nauseating, bitter fluid will be injected into your mouth, or nothing will happen.

Now imagine you are seated in the chair, with the electrodes attached to your right upper arm and the catheter inserted in your mouth. The light is waning and the first slide will soon appear on the screen. You may now fill in the questionnaire.

The thought experiment itself consisted of a questionnaire on which participants had to make UCS-expectancy judgments of the association between slides of certain animals and one of the three possible consequences: injection into the mouth of a nauseating but harmless drink (disgust-related outcome), an unpleasant but not painful electric shock (harm-related outcome) or no response at all. The animals were divided into three categories: high-predatory animals (pit bull terriers), low-predatory, high-disgusting animals (maggots) and neutral animals (rabbits). Spiders were included as an additional category to examine whether different UCSexpectancies would be observed between the high spider fearful group and the low spider fearful group.

For each of the slides, participants had to rate how many of these slides were to be followed by the drink, the shock, or by nothing at all on a $100 \mathrm{~mm}$ VAS, ranging from 0 ('none at all') to 100 ('all'). Thus, 12 UCS-expectancy judgments were obtained (4 animals $\times 3$ consequences). The sequence of these outcome ratings was fixed in a random order. First, participants had to rate the chance that slides of four animals (in this order: pit bull terriers, spiders, maggots and rabbits) were followed by an electrical shock. Next, ratings had to be given for the disgusting consequence, and finally, for expecting nothing. At the end of the questionnaire, participants also rated their fear of each of the four types of animals on a VAS scale ranging from 0 ('not afraid at all') to 100 ('very afraid').

\section{Procedure.}

On arrival at the lab, participants completed the Dutch translation of the FSQ. Next, the experimenter read aloud the introduction to the 'thought experiment' questionnaire, and showed one exemplary slide for each animal, after which the participants had to complete the questionnaire. Afterwards, they received a small reward for participating in the experiment. 


\section{Results}

\section{Data reduction and analysis}

To test the hypothesis that differential UCS-expectations exist for pit bull terriers, maggots and rabbits, expectancy ratings were subjected to a 3 (Animal Type: pit bull terriers, maggots, rabbits) x 2 (Outcome: harm-related consequence, disgust-related consequence) $\times 2$ (Spider Fear: high and low) ANOVA. Comparisons were made for the variable Outcome, in which the expectation of nothing was subtracted from the expectation of an aversive consequence (harmrelated: expectation of shock minus expectation of nothing; disgust-related: expectation of juice minus expectation of nothing).

Next, a series of post-hoc $t$-tests was conducted in order to specify which consequence was associated most strongly with each of the animals. For each animal, three $t$-tests were performed that compared the expectation of shock versus nothing, juice versus nothing and shock versus juice.

Finally, a regression analysis was used to study the relative contributions of the expectations of juice and shock to the fear of the three animals, (pit bull terriers, maggots and spiders). A VAS scale on animal fear (i.e., fear of pit bull terriers, maggots or spiders) was used as the dependent and both UCS-expectancies (shock and juice) as the predictor variables.

\section{UCS-expectations for pit bulls, maggots and rabbits.}

The 3 (Animal Type: pit bull terriers, maggots, rabbits) $\times 2$ (Outcome: shock minus nothing, juice minus nothing) $\times 2$ (Spider Fear: high and low) ANOVA revealed a main effect for Animal Type $(f(2,52)=79.94 ; p<.01 ; d=.75)$. This means that there was a difference between the animals to which they were associated with either of the aversive consequences. This is displayed in figure $1 \mathrm{a}$, which clearly shows that participants expected an aversive consequence to follow after the presentation of pit bulls and maggots but not after the slides of rabbits. The main effect for Outcome was not significant $(f(1,53)=2.99 ; p=.09 ; d=.53)$, indicating that, independent of animal type, both groups expected the aversive consequences (shock and juice) to occur equally often.

The crucial Animal Type $x$ Outcome interaction term was significant $(f(2,52)=29.01 ; p<$ $.01 ; d=.53$ ) which means that differential UCS-expectations existed for the animals in this study. The interaction term Animal Type $\mathrm{x}$ Outcome $\mathrm{x}$ Spider Fear was not significant $(F(2,52)$ $=1.25 ; p=.30 ; d=.05$ ), indicating that this differential pattern of associations was the same for low and high spider fear participants. In addition, none of the other interactions with spider fear, nor the spider fear main effect $(f 1,53)=.00 ; p<.95 ; d=.00)$ reached significance. This indicates that there were no significant differences in UCS-expectations for the three animals between low and high spider fearful individuals. 


\section{CHAPTER 9}

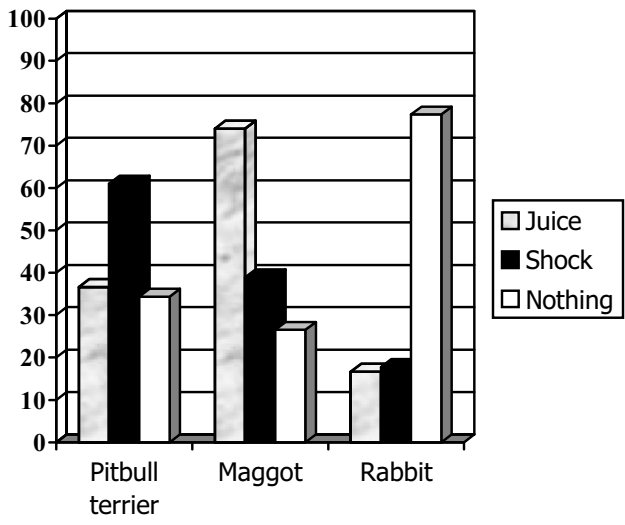

Figure 1a. UCS-expectancies for different animal types

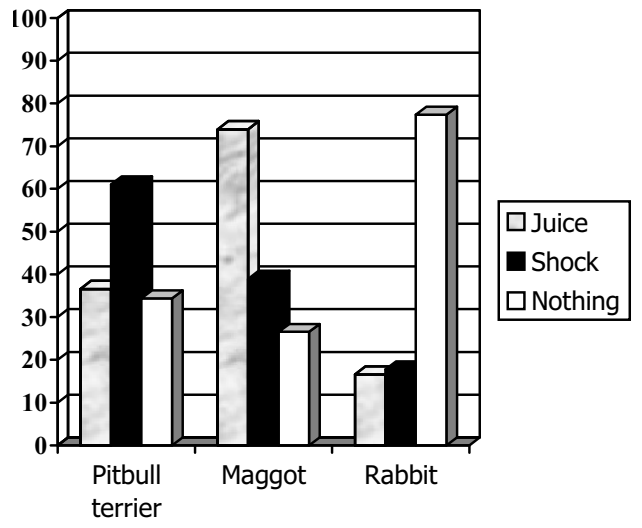

Figure 1b. UCS-expectancies for spiders in both groups

Subsequently, a series of post-hoc one-sample $t$-tests were performed, revealing that pit bull terriers were significantly more strongly associated with the expectation of the shock compared to the expectations of the juice $(t=6.88 ; p<.01)$ or with no consequence $(t=7.51 ; p<.01)$. For the pit bull terriers, no differences were observed between expectations of the juice and no consequence $(t=.69 ; p=.49)$. Maggots were significantly more strongly associated with juice compared to shock $(t=11.27 ; p<.01)$ or with expectations of nothing $(t=15.25 ; p<.01)$. Participants also expected a shock significantly more often than nothing after presentation of maggots $(t=3.62 ; p<.01)$. After the presentation of a rabbit, nothing was expected to occur significantly more often than either shock $(t=-4.31 ; p<.01)$ or juice $(t=-16.51 ; p<.01)$. The expectation of the juice and the expectation of the shock did not differ significantly after presentation of a rabbit $(t=-.46 ; p<.65)$.

\section{UCS-expectations for spiders.}

A 2 (Outcome: shock minus nothing, juice minus nothing) $\times 2$ (Spider Fear: high-fear, low-fear group) ANOVA was performed to examine whether the two groups differed in their UCSexpectations after hypothetical presentation of a spider slide. The intercept of this analysis was significant $(F(1,53)=35.04 ; p<.01 ; d=.40)$, which indicates that, in general, people expect an aversive consequence to occur with greater probability than a neutral consequence after the presentation of a spider slide. The strengths of the expectations of the aversive juice and the aversive shock were similar. Main effect Outcome was not significant $(f 1,53)=.79 ; p<.38$; $d=.02$ ). Most important for the present context, results did reveal a significant difference in the between-groups factor Spider Fear $(F 1,53)=12.56 ; p<.01 ; d=.19)$. This shows that although both groups expected an aversive consequence after imaginary presentation of a spider, this expectation was especially pronounced in the high spider fearful group. The inflated expectations in the high spider fear group were similar for both consequences, as was evidenced by the absence of a significant effect for the Outcome $\times$ Spider Fear interaction term $(f(1,53)=.30 ; p<.59 ; d=.01)$. 


\section{UCS-expectations and contributions to animal fear.}

Regression analyses were used to study the relative contributions of expectations of juice and shock to fear of the three animals (pit bull terriers, maggots and spiders). In the regression analyses, Animal fear (i.e., fear of pit bull terriers, maggots or spiders), as indexed by the VAS scales, was used as the dependent and both UCS-expectancies (shock and juice) as the predictor variables. Table 1 summarizes the results of the regression analyses. The analyses indicated that with respect to fear of pit bull terriers, the single best predictor was the expectation of a harm-related consequence $(\beta=.30 ; p=.03)$, while the disgust expectancy was irrelevant $(\beta=.02 ; p=.88)$. A second analysis indicated that a fear of maggots was not predicted by expectations of either harm-related consequences $(\beta=-0.03 ; p=.82)$ or disgustrelated consequences $(\beta=.05 ; p=.74)$. For spider fear, the single best predictor was the expectation of a disgusting response (juice) following presentation of the spider slides $(\beta=.29$; $p=.03)$. Shock expectancies had no independent predictive properties $(\beta=.17 ; p=.21)$.

Table 1.

Summary of the regression analyses for UCS-expectations and prediction of animal fear.

\begin{tabular}{|c|c|c|}
\hline Spider fear & $R^{2}$-sq & \\
\hline Variable & $\beta$ & $p$ \\
\hline Juice & .296 & .172 \\
\hline Shock & .03 & .21 \\
\hline Pit bull terrier fear & $R^{2}$-sq & \\
\hline Variable & $\beta$ & $p$ \\
\hline Juice & .021 & .302 \\
\hline Shock & .88 & .03 \\
\hline Maggot fear & $R^{2}-\mathrm{sq}$ & \\
\hline Variable & $\beta$ & $p$ \\
\hline Juice & .046 & .74 \\
\hline Shock & -.033 & .82 \\
\hline Rabbit fear & $R^{2}$-sq & \\
\hline Variable & $\beta$ & $p$ \\
\hline Juice & .332 & .21 \\
\hline Shock & -.110 & .68 \\
\hline
\end{tabular}

A series of bivariate correlations were conducted, to examine whether the fear of each animal (as measured with a VAS scale) would be significantly correlated with certain UCSexpectations (either the expectation of juice minus nothing or shock minus nothing). The results are displayed in Table 2. Significant correlations were observed between fear of pit bull terriers and the expectation of shock $(r=.30 ; p=.03)$, and between fear of spiders and the expectation of juice $(r=.35 ; p<.01)$. The correlation between spiders and the expectation of the shock approached significance $(r=.27 ; p=.05)$. The spiders were significantly negatively correlated with the expectation of no consequence $(r=-.43 ; p<.01)$, indicating that the more 


\section{CHAPTER 9}

fearful the participants were of spiders, the more they expected one of the aversive consequences. The same inverse correlation was found between the maggots and expectations of no consequence $(r=-.34 ; p=.01)$. Fear of maggots was not significantly correlated with expectations of any aversive consequence, indicating that people who are more fearful of maggots do not necessarily expect a specific consequence to occur. Rabbits were not significantly associated with any consequence.

Table 2.

Summary of bivariate Pearson's $p-m$ correlations for animal fear and UCS-expectations.

\begin{tabular}{lllll}
\hline & fear of pit bulls & fear of maggots & fear of rabbits & fear of spiders \\
\hline expectation of shock & $r=.30 *$ & $r=-.04$ & $r=.18$ & $r=.27$ \\
& $p=.03$ & $p=.80$ & $p=.20$ & $p=.05$ \\
expectation of juice & $r=.03$ & $r=.05$ & $r=.24$ & $r=.35 * *$ \\
& $p=.84$ & $p=.73$ & $p=.08$ & $p<.01$ \\
expectation of nothing & $r=-.24$ & $r=-.34 *$ & $r=-.07$ & $r=-.43 * *$ \\
& $p=.08$ & $p=.01$ & $p=.62$ & $p<.01$ \\
\hline
\end{tabular}

** is significant at the 0.01 level (2-tailed), * is significant at the 0.05 level (2-tailed)

\section{Discussion}

The major findings can be summarized as follows: i) evidence was found for differential UCSexpectations for different animals. More specifically, participants selectively associated a pit bull terrier (high-predatory animal) with a harm-related consequence, maggots (high-disgusting animals) with a disgusting consequence and a rabbit (neutral animal) with no consequence at all, ii) spiders were associated with aversive consequences (shock and juice) in both the low and high spider fearful groups, iii), although both groups expected an aversive consequence following a spider, the high spider fearful group expected significantly more often that either of the aversive consequences would occur, and finally, iv) the single best predictor of a fear of spiders was the expectation of a disgusting consequence, while the single best predictor of a fear of pit bull terriers was the expectation of harm-related consequences.

In line with previous research (Davey et al, 2003), the present data add to the evidence that differential UCS-expectancies exist for various groups of animals. The major goal of the present study was to investigate to what extent the expectations of disgust- and harm-related consequences are implicated in spider fear. In spider phobics, an expectancy bias has been found for the expectation of harm-related consequences (Cavanagh \& Davey, 2000; Kennedy et al., 1997; Öhman, Dimberg \& Öst, 1985). However, based on the accumulating evidence that the emotion of disgust contributes significantly to the existence and maintenance of spider phobia, the possibility arises that an expectancy bias towards disgusting consequences might also be relevant. Data from the present study reveal that the spiders were indeed associated with both aversive consequences (shock and juice) in both the high and low spider fearful groups, but significantly more strongly in the high spider fearful group. This evidence is in line 
with previous research, showing that spider phobics associate spiders with both harm-related and disgusting qualities (Davey, 1992b; de Jong \& Muris, 2002; Thorpe \& Salkovskis, 1998).

In addition, it was also studied which of the consequences would contribute most to spider fear. The data revealed that the expectation of a disgusting consequence was the strongest predictor of spider fear. Thus, results of this study are consistent with previous research (de Jong \& Muris, 2002; de Jong et al., 2002), which found that the perceived probability of involuntary physical contact with a disgusting object is a better predictor of spider fear than the perceived probability of being attacked (de Jong \& Muris, 2002). It seems that, independent of fear, all participants associate spiders with harm-related beliefs. Typically, the high spider fearful group also associates spiders more strongly with disgusting consequences than the low spider fearful group.

The bivariate correlations showed that, in line with a predator-defense explanation for a fear of pit bull terriers, a fear of fierce animals (pit bull terriers) was associated with inflated expectations of harm-related outcomes (shock) on pit bull trials. Fear of maggots was not associated with inflated expectancies of disgust-related outcomes on maggot trials. One testable explanation could be that fear of small animals is mainly determined by the interaction of disgust with the perceived likelihood of involuntary physical contact (de Jong \& Muris, 2002). For animals that are virtually universally considered as highly disgusting (e.g., maggots), the variability of fear is mainly determined by the variability in the perceived likelihood of involuntary physical contact. So, since most people consider maggots highly disgusting (as confirmed by the present study), a fear of maggots will be predominantly determined by the perceived probability of involuntary physical contact.

Since spider fearful individuals tend to have higher levels of disgust sensitivity (de Jong, Andrea \& Muris, 1997; Merckelbach et al., 1993; Mulkens, de Jong \& Merckelbach, 1996), it was expected that spider fearful individuals would not only associate spiders but also maggots relatively strongly with disgust-relevant outcomes. This was not the case. One explanation might be that people in general associate maggots already strongly with disgusting consequences, such that a ceiling effect prevents disgust sensitivity to further contribute to people's expectancy that maggots will be followed by disgust-relevant outcomes.

For spiders, the results revealed a different pattern. Here, the expectation of disgust-related consequences following their presentation was significantly stronger in the high spider fearful group compared to the low fearful group. It would be interesting to examine in future research whether this association between spider fear and disgust-relevant outcome expectancies is causal in nature. If indeed disgust-relevant outcomes expectancies are causally related to a fear of spiders, then priming or inflating disgust-relevant outcome representations should enhance the experience of spider fear. Preliminary evidence suggests this might be the case. In a study by Webb \& Davey (1992), participants saw either a threat-related movie (i.e., extreme violence) or a disgusting movie (i.e., a surgery), after which they saw a slide show featuring animal slides. They had to rate how afraid they were of each animal. Results showed that participants who saw the threat-related movie were significantly more afraid of predatory animals, and participants who saw the disgusting movie were significantly more afraid of disgusting animals (Webb \& Davey, 1992). A second indication that expectancy bias might be causally involved in 


\section{CHAPTER 9}

spider phobia was found by de Jong, van den Hout and Merckelbach (1995). They showed that treated spider phobics who showed a residual bias to overestimate the covariation between spiders and harm-relevant outcomes had a relatively greater chance of a return of phobic fear complaints after two years of follow-up. Results of the present study suggest that a residual expectancy bias towards disgusting consequences might be relevant as well.

The present study even reveals that expectations of disgust-related consequences following spiders are at least equally relevant in spider fear as expectations of harm-related consequences. It is not illogical that spiders are also associated with disgust-relevant consequences. Most spider species known in European countries are completely harmless (Davey, 1994a), and only a very small fraction of all known spiders species do actually possess lethal qualities (Roberts, 1995). However, throughout history, spiders have become linked with the spread of numerous diseases. In 1307 in southern Italy, a strange disease occurred called 'Tarantism' after people were bitten by spiders (Russell, 1979). In the middle ages, spiders became associated with the black plague (de Jong, 1996) and even today, diagnoses of several diseases that follow from a bite by the brown recluse spider greatly outnumber their presence in the USA (Vetter, Cushing, Crawford \& Royce, 2003). Therefore, it has been suggested that a fear of small animals, such as spiders, can be related better to a disease-avoidance model than a predator-defense model (Matchett \& Davey, 1991). The disease-avoidance model postulates that spiders would be associated with their potential to spread disease, dirt and contamination. By being disgusted of spiders, people would want to avoid physical contact, thus, protecting people against diseases. Spiders would acquire such a disgust-evoking status by means of three possible routes: 1 ) by directly spreading disease, 2 ) by being contingently associated with dirt or contamination, or 3) by resembling prototypical disgust stimuli (Davey, 1992).

The finding that disgust-related expectations can be observed in spider fearful individuals following presentation of a spider not only supports the idea that disgust may contribute to the occurrence of spider phobia, it may also have important implications for treatment. To make the treatment for spider phobia more successful, it would be advisable to reduce the expectancy bias for both components (expectation of harm-related and disgust-related consequences). This could be done by addressing not only the harm-related beliefs people may hold about spiders, but their disgust-evoking status as well (de Jong, Vorage, van den Hout, 2000).

Finally, some comments are in order with respect to several limitations in the present study. First, the current study used non-clinical participants who displayed an extreme fear of spiders, as measured with the FSQ. Although differences in UCS-expectancies were observed between the high and low spider fearful group, the data did not reveal significant differences between harm- and disgust expectancies in the high fearful group. Both aversive consequences were expected significantly more often following presentation of a feared stimulus. It could be argued that individuals with clinical levels of spider fear might be more focused on aspects of harm than on the possibility of contamination with a disgusting object. Although the results of the present study are in line with previous research using analogue groups (de Jong \& Muris, 2002; de Jong, Peters \& Vanderhallen, 2002; Woody \& Tolin, 2002), it cannot be ruled out that a treatment-seeking sample will be characterized by a different expectancy bias. 
Second, this study has only included female participants. It is known that women tend to have higher levels of disgust sensitivity than men (Davey, 1994b; Ware et al., 1994). This may have resulted in higher baseline-measurements of expectations of disgust consequences following disgust-relevant animals. It could be that compared to a sample that does have an equal distribution of males and females, the UCS-expectations for disgust consequences following spiders in the present study are higher.

Third, it was established that highly spider fearful individuals displayed relatively strong expectancies for both harm and disgust-relevant outcomes following spider slides. Expecting either of these types of consequences may motivate phobics to avoid spiders, and thus, to avoid potentially disconfirming information. However, recently it has been shown that even if people obtain disconfirming information, they still persist in their dysfunctional expectations (de Jong \& Merckelbach, 2000; Pauli, Montoya \& Martz, 1996, 2001). Therefore, it would be important to examine this relative insensitivity to disconfirming information to determine which of the expectations is most strongly associated with fear of spiders. Hence, it would be interesting to subject both high and low spider fearful individuals to an illusory correlation paradigm including both types of outcomes to examine for both types of expectancy biases the relative (in)sensitivity to corrective information.

Additionally, an implication of the present study could be that an expectancy bias for disgust-related consequences may contribute to the etiology and maintenance of several other disgust-relevant disorders as well, such as eating disorders (Troop et al., 2000; Troop, Treasure \& Serpell, 2002), obsessive-compulsive disorder (Mancini, Gragnani \& D'Olimpio, 2001; Woody \& Tolin, 2002), and blood phobia (Sawchuk et al., 2002). Also, studying expectations of both harm-related and disgust-related consequences could provide an explanation as to why previous studies found no expectancy bias for these disorders. For instance, in a study by Pury \& Mineka (1997), both high and low blood fearful participants overestimated the co-occurrence of a harm-related consequence following fear-relevant slides. More importantly, no differences were observed between the high and low fearful groups. It could be, that although no expectancy bias for harm-related consequences (the shock) was apparent, an expectancy bias for disgust-related consequences (juice) might well be found. 
CHAPTER 9

\section{Appendix}

These stimulus pictures were used as exemplars

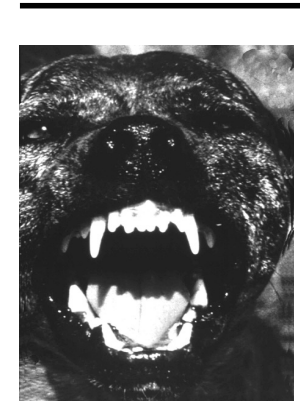

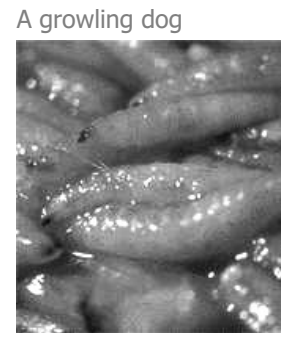

Maggots
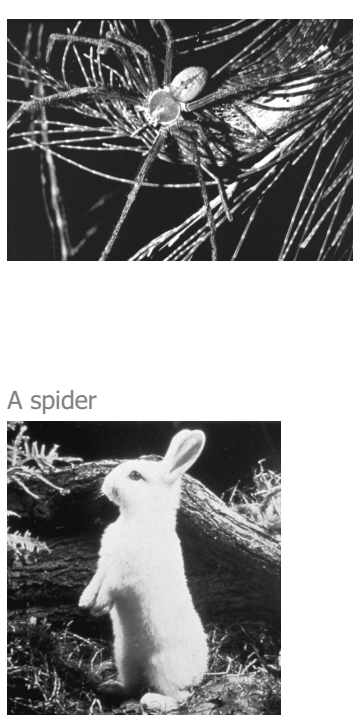

A rabbit

Note. The original slides were in color. 


\section{Chapter 10}

\section{Disgust and fear related UCS-expectancy bias in blood fearful individuals}

'On a moonlight night you got yer dead toad frog

Got yer dead rabbit and yer dead raccoon The blood and the guts they're gonna make you swoon

You got yer dead skunk in the middle of the road Stinkin' to high heaven!' Loudon Wainwright III 


\section{CHAPTER 10}

\section{Abstract}

People with specific fears tend to overestimate the occurrence of unpleasant consequences (e.g., electrical shock) following presentation of feared objects. Increasing evidence suggests that blood phobia is qualitatively different from other specific phobias since confrontation with phobic stimuli involves disgust and repulsion rather than (threat-induced) fear. Therefore, this study examined the role of disgust-related UCS-expectancies following confrontation with blood phobia-relevant stimuli. High $(n=30)$ and low $(n=30)$ blood fearful individuals estimated the probability that hypothetical presentation of slides containing a rabbit, maggots, a dog, a weapon, or a bloody wound would be followed by a sip of nauseating juice, a fear-related electrical shock, or nothing. Although participants generally expected shock and juice following blood, UCS-expectancies for both aversive outcomes following blood were significantly more pronounced in high blood fearful participants. This implicates that UCS-expectancy biases may also be involved in the development and maintenance of blood phobia. 
Individuals with specific phobias hold strong convictions that catastrophic consequences occur upon confrontation with phobic stimuli and tend to overestimate the predictive relationship between phobic stimuli (CS) and aversive outcomes (UCS) (UCS-expectancy bias; Davey, 1992). Accordingly, laboratory studies show that, for example, spider phobics are characterized by a relatively strong expectancy bias for aversive UCS-outcomes (e.g., electrical shock) following spiders (e.g., Davey, 1992; van Overveld, de Jong, \& Peters, 2006). Similar findings have been observed in panic disorder (Wiedemann, Pauli, \& Dengler, 2001), and snake phobia (McNally \& Heatherton, 1993). In apparent conflict with the idea that associative biases are generally involved in the maintenance of specific phobias, a series of experiments in the context of bloodinjection-injury (BII) fears showed that the tendency to selectively associate BII-relevant stimuli with aversive outcomes was not especially pronounced in BII-fearful individuals (Pury \& Mineka, 1997).

However, studies on phobia-relevant associative biases (including the experiments by Pury and Mineka) predominantly focused on fear-related UCS-outcomes (electrical shock or loud tone). Meanwhile, in addition to fear-related preoccupations disgust seem to be critically involved in blood phobia (Page, 1994; Sawchuk et al., 2000). Indirect evidence for this comes from studies showing that blood fearful individuals systematically display enhanced levels of disgust propensity (de Jong \& Merckelbach, 1998; Sawchuk et al., 2000) and disgust sensitivity (van Overveld et al., 2006). Studies directly testing individuals' emotional responses upon confrontation with blood-relevant materials underline the role of disgust in blood fears: blood fearful individuals report primarily to experience disgust when confronted with BII-stimuli (Sawchuk et al., 2000; Tolin, Lohr, Sawchuk, \& Lee, 1997). Since affective similarity between stimuli and outcomes seems an important factor in generating associative biases (e.g., Tomarken et al., 1995), inflated expectancies of outcomes that elicit disgust rather than fear may be primarily involved in blood phobia. If so, this may explain the absence of relatively strong associative biases in BII-fearful individuals in previous research using fear-related rather than disgust-related aversive outcomes (Pury \& Mineka, 1997).

In a first exploration of this, de Jong and Peters (2007a) followed the thought experiment procedure of McNally and Heatherton (1993). High and low blood fearful individuals estimated which part of a series of hypothetical slides containing certain stimuli (i.e., rabbits, flowers, and blood-donation scenes) would be followed by a disgust-relevant outcome (a sip of disgusting juice), a fear-relevant outcome (electrical shock), or a neutral outcome (nothing), if they were to participate in such an experiment. Participants generally expected aversive outcomes (shock and juice) following blood slides, but in apparent conflict with the hypothesis that disgustrelated associative biases would be especially pronounced in fearful individuals, no differences emerged between groups (or outcomes).

However, the absence of significant differences between high and low fearful individuals might have been caused by having only one category of salient negative stimuli (i.e., blooddonation slides) within the design. In the absence of concurrent negatively valenced stimuli, participants may have generally associated the (single) category of slides with negative connotations (i.e., blood-slides) with the aversive outcomes (cf. de Jong, Merckelbach, Arntz, \& Nijman, 1992). Therefore, in the present study, two slides were included as a concurrent 


\section{CHAPTER 10}

category of negative slides and which were clearly prototypical of the investigated emotions, namely fear (a gun pointed at the viewer) and disgust (maggots). Adding such stimuli renders the stimuli of interest less salient and more ambiguous as to the stimulus/outcome associations. Thus, the presence of these competing negatively valenced stimuli is likely to undermine the UCS-expectancy bias for blood in low blood fearful individuals, thereby increasing the sensitivity of the procedure to detect differential UCS-expectancy biases (cf. de Jong et al., 1992).

Another feature of the study by de Jong and Peters (2007a) that might have blurred their results concerns their operationalisation of UCS-expectancy bias. They asked participants to estimate which part of all slides would be followed by the various consequences throughout the entire experiment (expectancies of covariation; e.g., McNally \& Heatherton, 1993). Such a covariation rating task is quite abstract and easily misunderstood (cf. Amin \& Lovibond, 1997). These features may well have introduced noise, thereby undermining further the sensitivity of the procedure to find individual differences in UCS-expectancy bias. Therefore, the present study adopted a more simple and straightforward procedure. Participants were asked to rate the probability that a particular outcome would occur following a specific slide that was representative for a certain category (see Davey, Cavanagh, \& Lamb, 2003). Instead of estimating covariation expectancies for the full experiment, participants only imagined what they believed would happen for that specific slide at that particular moment.

In short, the present study tested the following issues: (1) are high blood fearful individuals characterized by UCS-expectancy biases, and (2) are enhanced expectancy biases (if present) specific for disgust-outcomes?

\section{Methods}

Participants.

Students at the faculties of Medicine, Health Sciences, and Psychology at Maastricht University were recruited via posters, and advertisements in the university buildings. Eventually, a total of 212 students completed the Blood-subscale of the Medical Fear Survey (MFS; Kleinknecht, Thorndike, \& Walls, 1996). The 30 highest and 30 lowest scoring individuals were invited to participate. As the majority of students at these faculties are women, the research population consists predominantly of women $(86.7 \%)$. Mean age was 22.9 years $(S D=6.86)$. Participants were classified as high blood fearful, if two of the following criteria were met: a) they rated themselves at least 7 on a scale from 1 (= not blood fearful at all) to 10 (=extremely blood fearful), b) MFS-Blood > 5, and c) Blood-Injury Phobia Questionnaire-Fear subscale (BIQ-Fear; de Jong \& Merckelbach, 1998) > 20. The participants were classified as low blood fearful, if they: a) rated themselves non-blood fearful $(<5)$, b) MFS-Blood $<5$, c) BIQ-Fear $<18$. 


\section{Materials}

Disgust Scale (DS; Haidt et al., 1994). The DS measures disgust propensity across eight domains: Body-Envelope Violations, Animals, Bodily Products, Hygiene, Sex, Food, Death, and Magical Thinking. The first part contains 16 true/false type questions. Participants rate their agreement to 16 statements (e.g., 'I think homosexual activities are immoral.'). The second part inquires how disgusting participants consider 16 experiences (e.g., 'You hear about an adult woman who has sex with her father.') using a scale from 0 (= 'not disgusting at all') to 2 ( $=$ 'very disgusting'). Internal consistencies are poor for the subscales $(a=.34-.64$, Haidt et al., $1994 ; .33-.74$, present study), but high for the total score ( $a=.84$, Haidt et al., 1994; .85, present study).

Disgust Propensity and Sensitivity Scale-Revised (DPSS-R; van Overveld et al., 2006). This index measures disgust propensity, which is how rapidly one experiences disgust, and disgust sensitivity, which is how unpleasant the disgust experience is to the individual (Cavanagh \& Davey, 2000). Individuals rate their agreement with 16 propositions concerning the frequency of certain bodily symptoms (e.g., 'I find something disgusting'), and their emotional impact (e.g., 'When I am disgusted, I am worried that I might pass out) on a scale from 1 (= 'never') to 5 (= 'always'; range: $16-80$ ). Both subscales propensity ( $a=.69$, van Overveld et al., 2006; .87 , present study) and sensitivity ( $a=.71$, van Overveld et al., 2006; .82, present study) show satisfactory reliability.

Blood-Injury Phobia Questionnaire (BIQ; de Jong \& Merckelbach, 1998); The BIQ examines fear of blood and fainting history using two 10-item subscales. On the first part, BIQ-fear, participants rate their fear of ten blood-items (e.g. 'Please rate how afraid you are of: 1. blood, 2. hospitals, etc.') on a scale from 0 (= 'no fear') to 4 (= 'maximal fear'; range $=0-40)$. The BIQ-Fear is reliable (.82-.87, Merckelbach, Muris, de Jong \& de Jongh, 1999; .91, present study). On the second part, BIQ-Fainting, participants rate their fainting history for the BIQFear items (e.g., 'Please rate how often you have fainted in the presence of: 1 . blood, etc.') on a scale from 0 (= 'never') to 2 (= 'often'; range: 0 -20). Internal consistency of the BIQ is satisfactory ( $a=.73-.78$, Merckelbach et al., 1999; .81, present study).

Medical Fear Survey (MFS; Kleinknecht, Thorndike, \& Walls, 1996); This questionnaire assesses fear of medical stimuli using five 10-item subscales (Mutilation, Sharp Objects, Examinations and Symptoms as Intimation of Illness, Injections and Blood Draws, Blood). Participants rate whether a series of statements are typical of them on a scale from 0 (= very slightly) to 4 (= extremely). The subscales are reliable ( $a=.84-.94$, Kleinknecht, Kleinknecht, Sawchuk, Lee \& Lohr, 1999; .87 - .97, present study)

Multidimensional Blood / Injury Phobia Inventory (MBPI; Wenzel \& Holt, 2003); This questionnaire measures fear of blood, covering a broad domain of stimuli and responses. It contains 40 items on 4 types of stimuli (injections, hospitals, blood, injury) and 5 possible types of responses (fear, avoidance, worry, fainting, disgust). Participants rate the degree to which each item is typical of them on a scale from 0 (= very slightly or not at all) to 4 (= extremely). A total score and six subscales (Injections, Hospitals, Fainting, Blood-Self, Injury, Blood/Injury- 


\section{CHAPTER 10}

others) can be calculated. Internal consistency is high for both total score $(a=.91$; Wenzel \& Holt, 2003; .97, present study) and subscales ( $a=.86-.96$, present study).

\section{Procedure}

Upon arrival at the lab, participants completed the DS, DPSS-R, BIQ, MFS, MBPI. Next, they received a verbal introduction and a binder containing the UCS-questionnaire (introduction in Appendix).

In accordance with previous studies (Davey et al., 2003; van Overveld et al., 2006), slides of high-disgusting animals (maggots), high-predatory animals (growling dog), and neutral animals (rabbit) were used to test whether inflated expectancy biases in high blood fearful individuals (if present) are specific to blood or represent a generally enhanced UCS-expectancy bias. Further, although dogs are primarily associated with fear and physical harm, they also elicit disgust-relevant associations (Huijding, 2006). Therefore, a slide of a gun pointed at the viewer was included as an additional and fear-relevant category. To determine UCSexpectancies for blood, a blood slide was included.

In the binder, exemplary color slides*** of each stimulus were presented on the page prior to the page containing questions regarding that stimulus. So, on the first page, participants would read: 'The following slide appears: maggots' together with an exemplary slide. On the next page, participants would read: 'Imagine that during the experiment you will be shown the following slide: maggots. What do you think the chances are that this slide depicting maggots will be followed by an electrical shock?' This question was repeated for all outcomes (i.e., juice, nothing). Ratings were obtained using a 100-mm VAS from 0 ('very small') to 100 ('very large'). The sequence of ratings was fixed in a random order, and resulted in 15 UCS-expectancy ratings per person ( 5 slides $\times 3$ outcomes) pertaining to the probability that the slides (in this order: maggots, rabbit, blood, dog, weapon) would be followed by a particular outcome (in this order: shock, juice, nothing). After completion, participants received a small financial incentive.

\section{Results}

\section{Statistical analyses}

Missing values were estimated using regression analyses following recommendations of Schafer \& Graham (2002). Independent sample $t$-tests examined whether low and high fearful groups differed on all questionnaires (MFS, BIQ, MBPI, DS, DPSS-R). To examine if UCS-expectations for blood differ as a function of group, ANOVAs with within-subjects factor outcome type (shock minus nothing, juice minus nothing) and between-subjects factor blood fearful (high, low) were used. Similar ANOVAs for the other stimuli determined whether a possible expectancy bias amongst blood fearful individuals is specific to blood, or indicates a heightened overall UCS-

*** The exemplary slides were obtained from the International Affect Picture System (IAPS numbers: 1300, 1610, $6230)$, except the slides depicting a small bloody wound and maggots. All slides are presented in the Appendix. 
expectancy bias. Subsequently, to determine which outcome was most strongly associated with each stimulus, three post-hoc $t$-tests were performed (comparing expectation of shock versus nothing, juice versus nothing, and shock versus juice).

\section{Descriptives}

Table 1 shows that, as expected, the high blood fearful group scored significantly higher on fear of blood (BIQ, MFS, MBPI), disgust propensity (DS, DPSS-RP) and disgust sensitivity (DPSS-RS).

Table 1.

Mean scores on questionnaires for high and low blood fearful participants

\begin{tabular}{lllll}
\hline Indices & High fear & Low fear & $t(58)$ & $p$ \\
\hline DS total & $19.55(3.97)$ & $14.02(3.87)$ & -5.46 & $<.01$ \\
DPSS-R & & & \\
Propensity & $23.70(4.40)$ & $17.90(3.14)$ & -5.87 & $<.01$ \\
Sensitivity & $19.63(4.94)$ & $13.80(3.21)$ & -5.43 & $<.01$ \\
BIQ-Fear & $27.83(6.53)$ & $14.70(3.20)$ & -9.89 & $<.01$ \\
BIQ-Faint & $11.37(2.17)$ & $10.10(.40)$ & -3.14 & $<.01$ \\
MFS & & & & $<.01$ \\
Injections & $17.77(8.49)$ & $3.27(3.34)$ & -8.70 & $<.01$ \\
Sharp Objects & $10.47(7.64)$ & $4.13(3.16)$ & -4.20 & $<.01$ \\
Disease & $11.47(5.47)$ & $6.07(3.56)$ & -4.53 & $<.01$ \\
Mutilation & $21.27(8.15)$ & $6.70(4.79)$ & -8.44 & $<.01$ \\
& & & & $<.01$ \\
Blood & $13.27(7.37)$ & $1.20(1.85)$ & -8.70 & $<.01$ \\
MBPI total & $63.50(23.11)$ & $8.30(8.67)$ & -12.25 & $<.01$ \\
Injections & $13.70(5.79)$ & $1.23(2.73)$ & -10.67 & $<.01$ \\
Hospitals & $5.00(4.39)$ & $.83(1.56)$ & -4.90 & $<.01$ \\
Fainting & $4.00(4.71)$ & $.27(.94)$ & -4.26 & $<.01$ \\
Blood-self & $9.17(4.36)$ & $.33(.66)$ & -10.96 & -6.61 \\
Injury & $8.23(4.07)$ & $2.53(2.40)$ & & \\
\hline Note. Standary & & & & \\
\end{tabular}

Note. Standard deviations are described in parentheses

\section{UCS-expectations for blood}

A 2 (Outcome: shock minus nothing, juice minus nothing) $\times 2$ (Blood Fear: high or low) ANOVA was performed. The significant intercept $(f 1,58)=18.13 ; p<.01 ; d=.24)$ indicates that overall, participants expected aversive outcomes following the blood slide. There was no main effect of Outcome $(\neg 1,58)=.42 ; p=.84 ; d<.01)$, so generally, the participants expected shock and juice equally strongly. Yet, the main effect of Group was significant $(F 1,58)=4.46$; $p=.04 ; d=.07$ ) indicating that high blood fearful participants expected aversive outcomes more strongly than low blood fearful participants (see Figure 1). This inflated outcome expectancy was similar for both outcome types as interaction term Outcome $x$ Group was not significant $(F(1,58)=0.18 ; p=.68 ; d<.01)$. 


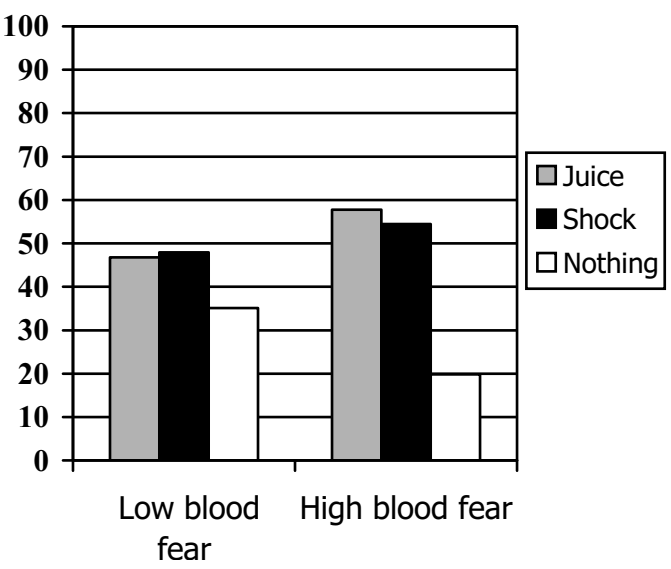

Figure 1. UCS-expectancies for the blood slide in high and low blood fearful participants.

\section{UCS-expectations for the other stimuli.}

A 4 (Slide Type: rabbit, dog, maggots, weapon) $\times 2$ (Outcome: shock minus nothing, juice minus nothing) $\times 2$ (Blood Fear: high, low) ANOVA revealed a main effect for Slide Type $(F(3,56)=33.35 ; p<.01 ; d=.64)$ showing that some slides were associated with aversive outcomes more strongly than others. The main effect for Outcome was significant $(\Re(1,58)=$ 50.43; $p<.01 ; d=.46$ ) indicating that, overall, participants expected shock more often than juice. The main effect of Group was not significant $(f 1,58)=0.16 ; p=.69 ; d<.01)$, so no differences were observed between groups in expectations of aversive outcomes in general.

In line with our expectations, interaction term Slide Type $\mathrm{x}$ Outcome was significant $(F(3,56)$ $=32.97 ; p<.01 ; d=.64$ ), indicating that type of UCS-expectancy bias varied as a function of stimulus type. Most importantly, this effect was similar for both groups, as indicated by nonsignificant interaction term Slide Type $\mathrm{x}$ Outcome $\mathrm{x} \operatorname{Group}(F(3,56)=1.13 ; p=.34 ; d=.06)$. Post-hoc paired samples $t$-tests showed that participants associated the rabbit significantly more strongly with nothing compared to outcomes juice $(t(59)=-11.74 ; p<.01)$ or shock $(t(59)=-6.252 ; p<.01)$, and stronger with shock than juice $(t(59)=4.38 ; p<.01)$. Maggots were associated most strongly with juice, compared to shock $(t(59)=-7.53 ; p<.01)$ or nothing $(t(59)=5.85 ; p<.01)$. There were no differences in expectations of shock versus nothing $(t(59)=.56 ; p=.58)$. The dog was associated significantly stronger with shock, compared to the juice $(t(59)=8.53 ; p<.01)$ or nothing $(t(59)=6.27 ; p<.01)$. There were no differences between UCS-expectations of juice versus nothing $(t(59)=-.88 ; p=.38)$. The weapon was associated significantly stronger with shock, compared to juice $(t(59)=7.23 ; p<.01)$ or nothing $(t(59)=4.09 ; p<.01)$. There was a trend towards a stronger association nothing than with juice $(t(59)=-1.83 ; p=.07)$. Thus, the rabbit was most strongly associated with nothing, maggots with juice, and dog and weapon with shock (see Figure 2). 


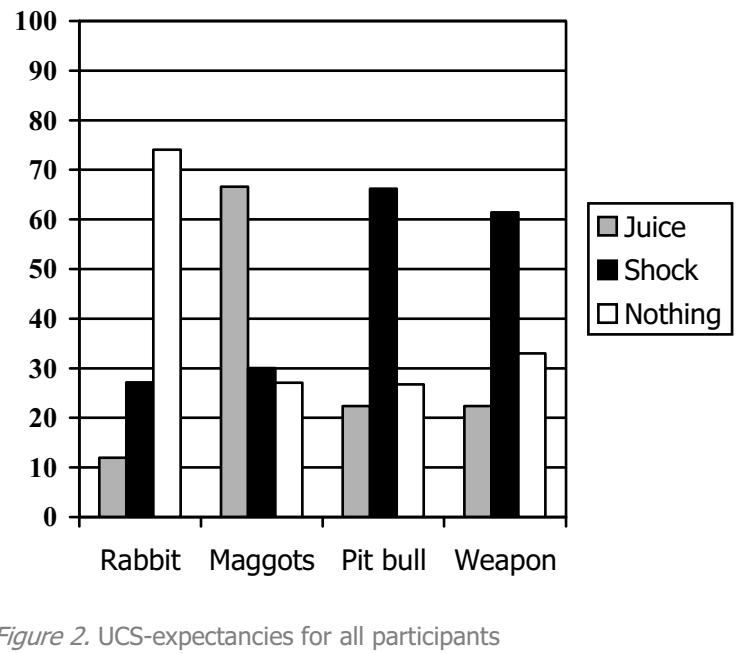

\section{Discussion}

The main findings are: i) participants generally expected both fear- and disgust-related outcomes following blood; ii) UCS-expectancies for aversive outcomes following blood were most pronounced in high blood fearful individuals; iii) replicating previous research, differential outcome expectancies were evident for prototypical disgust- and fear-related stimuli in all participants.

Corroborating earlier work (Davey et al., 2003; van Overveld et al., 2006), differential UCSexpectancies were observed. More specifically, participants associated dog and weapon with a feared outcome (shock), maggots with a disgust-related outcome (juice), and rabbit with a neutral outcome (nothing). Most importantly, only UCS-associations for the blood slide differentiated between groups. High blood fearful individuals expected both aversive outcomes following blood significantly more strongly than low blood fearful individuals. This sustains the notion that blood fearful individuals are characterized by an UCS-expectancy bias.

The finding that high blood fearful individuals display a UCS-expectancy bias for bloodrelated stimuli contrasts with earlier work by de Jong and Peters (2007a). In that study, high and low blood fearful participants generally expected aversive outcomes (shock and juice) following blood slides, but no differences emerged between groups. However, by using only one salient negative stimulus (i.e., blood-donation slides) in their experiment, participants may have generally associated the (only) category of slides with negative connotations (i.e., blood-slides) with the aversive outcomes (cf. de Jong, Merckelbach, Arntz, \& Nijman, 1992). Therefore, in the present study, two slides were included which were prototypical of fear (a gun) and disgust (maggots) as a concurrent category of negative slides. The present results are consistent with the prediction that this would undermine the UCS-expectancy bias for blood-relevant stimuli in low fearfu/ individuals. Thus, the inclusion of additional salient negative stimuli indeed increased the sensitivity of the procedure, and suggests that the absence of differential UCS-expectancy 


\section{CHAPTER 10}

biases in the study by de Jong and Peters (2007a) might have been the result of methodological issues (i.e., inflated UCS-expectancies for blood-slides in their control group by using only blood-related slides as salient negative stimuli).

UCS-expectancies for both aversive outcomes were equally strong, suggesting that fear- and disgust expectancies may be equally important to blood phobia. However, the fear-related outcome expectations might have been inflated by the use of an exemplary slide depicting a small, bloody wound. That is, the fear-related outcome expectancies may partially be the result of a close semantic connotation between a (painful) wound and a conceptually related outcome (i.e., painful electrical shock). Therefore, expectations of specifically fear-related outcomes may be reduced when using an exemplary slide depicting only blood. Nevertheless, although UCSexpectancies for both aversive outcomes were equally strong in blood-fearful individuals, the current findings clearly support the view that enhanced UCS-expectancy biases appear a universal phenomenon in phobic complaints.

The finding that blood fearful individuals display a disgust and fear-related UCS- expectancy bias is an important first step in determining whether this type of expectancy bias is indeed involved in the maintenance of the phobic complaints. It should be acknowledged, however, that although the available evidence is consistent with the alleged reciprocal relationship between blood fear and UCS-expectancy bias (e.g., Davey, 1997), it can not be ruled out on the basis of the present findings that UCS-expectancy biases are mere epiphenomena of (blood) fears. While causality problems of the present type are hard to solve, they are theoretically very important. One way to further explore this causality issue would be to see how UCS-expectancy bias is affected by successful treatment. If UCS-expectancy bias plays a critical role in the maintenance of complaints, it should be substantially reduced after successful treatment. Moreover, a critical implication of the proposed reciprocal relationship between complaints and expectancy bias would be that successfully treated patients who nevertheless show a posttreatment bias are more vulnerable to relapse than patients who do not display such posttreatment bias (cf. de Jong et al., 1995). Finally, the most rigorous way to test the causal properties of UCS-expectancy bias would be to specifically reduce UCS-expectancy bias and to test whether this results in a reduction of blood fear (cf. MacLeod, Rutherford, Campbell, Ebsworthy, \& Holker, 2002).

If UCS-expectancy biases are indeed involved in the maintenance of psychopathological symptoms, then it could be that differential UCS-expectancy biases are relevant to specific types of psychopathology. Thus, expectations of disgusting outcomes may be more relevant in disorders where disgust is hypothesized to be predominantly involved, such as blood phobia (Page, 1994), whereas for disorders where fear appears the primary motor of phobic distress (e.g., dog phobia), fear-related expectancies may be relatively more important. Additionally, it would be interesting to investigate whether treatment can be enhanced by focusing on specific UCS-expectancies and belief systems regarding phobic objects. Furthermore, if (residual) UCSexpectancy biases indeed undermine the consolidation of treatment effects (cf. de Jong, van den Hout, \& Merckelbach, 1995), it may be of clinical significance to signal such biases in a preliminary stage in order to prevent relapse. 
Further, several points should be addressed in future research. First, as the high blood fearful participants lacked a clinical diagnosis of blood phobia, it may be questioned whether this group was genuinely blood-fearful. However, scores on indices of blood fear were significantly different between low and high blood fearful groups. More importantly, scores of high fearful participants resembled earlier reports using clinical populations (Kleinknecht, 1993; Wenzel \& Holt, 2003). Future research should nevertheless investigate if similar or more pronounced results emerge when using a treatment-seeking sample.

Second, the present study relied on a thought experiment procedure. Although such a procedure is widely used in research on expectancy bias, it should be acknowledged that participants' ratings when asked to predict which outcome will follow the presentation of a particular slide in an imaginary experiment may be influenced by demand characteristics. Most important, it can not be ruled out that the results partly reflect participants' guesses of the experimenter's intentions rather than their own judgments about blood-outcome associations. It would therefore be important to verify whether a similar pattern of outcome expectancies would emerge during actual encounters with blood-related stimuli in an actual experiment (cf. de Jong \& Peters, 2007b). Following this, it would be interesting for future studies to expose high and low blood fearful individuals to an actual illusory correlation (IC) experiment (e.g., de Jong \& Peters, 2007a), rather than to an imaginary experiment. Such a procedure also allows exploring whether initial disgust and fear-related outcome expectancies vary with respect to their sensitivity to corrective experiences. The relative (in)sensitivity to disconfirming information may provide important clues with respect to the importance of disgust-relevant and fearrelevant UCS representations in blood phobia. 


\section{CHAPTER 10}

\section{Appendix}

\section{Introduction}

This brief questionnaire studies how people perceive experimental research. You are about to participate in a thought experiment. This means that we will ask you to imagine a specific situation as vividly as possible. After this, you will be asked to complete a questionnaire. You will now receive a short description of an experiment, after which you are to imagine being a participant in that experiment.

\section{Description of the experiment}

You are invited here to participate in an experimental study at the laboratory. You will be asked to sit down in a comfortable chair, and view a series of slides, projected on a large screen. Two electrodes will be placed on your upper arm and before the experiment starts a level of electrical shock will be selected in consultation with you. This will be done so the level is certainly unpleasant, yet not painful. During the experiment you will receive shocks at certain moments. Also, a catheter will be inserted in your mouth, and taped to your cheek, so at certain moments a fluid can be injected. This fluid tastes very bitter, and is quite nauseating. The fluid is, however, just like the shock, unpleasant yet harmless and without side effects.

During the experiment, you will view a series of slides of five different categories: maggots, rabbits, blood, dogs, and weapons. Each slide is presented for exactly six seconds and is immediately followed by one of three consequences: either you will receive a short but unpleasant electrical shock, or a shot of the nauseating, bitter fluid will be injected into your mouth, or nothing will happen.

Now imagine that you are seated in the chair, with electrodes attached to your right upper arm and a catheter inserted in your mouth. The light is waning and the first slide will soon appear on the screen. You may now complete the questionnaire. 


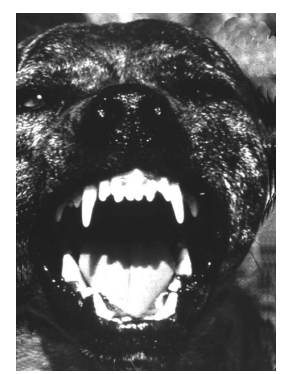

Growling dog

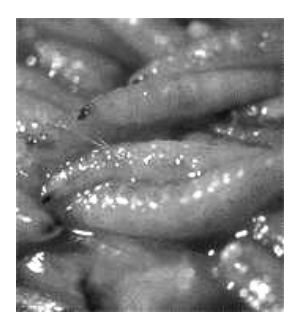

Maggots

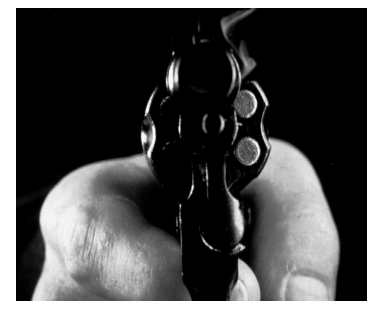

A weapon

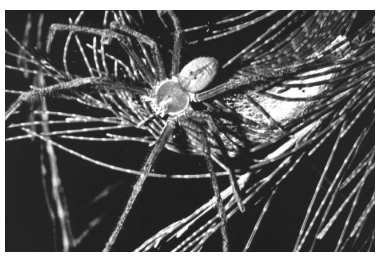

A spider

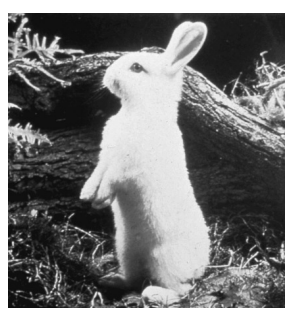

A Rabbit

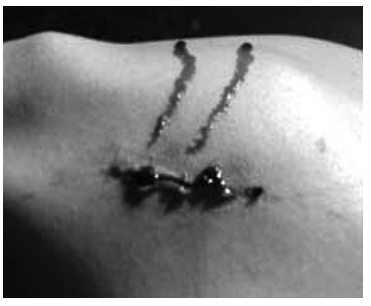

A bloody wound

Note. The original slides were in color. 
Chapter 11

Contamination and harm relevant UCS-expectancy bias in spider phobic individuals: influence of treatment and its relationship with the return of fear 


\section{CHAPTER 11}

\section{Abstract}

Phobic individuals expect aversive UCS's following encounters with phobic stimuli. Contamination rather than harm-related outcome expectancies differentiated best between high and low spider fearful individuals (van Overveld et al., 2006). This study investigated the proposed reciprocal relationship between these types of biased outcome expectancies and spider fear and tested whether: a) treatment seeking spider fearful individuals $(n=60)$ displayed inflated contamination- and harm-related UCS-expectancies compared to low spider fearful participants $(n=30)$; b) biased expectancies disappear following successful treatment, and c) residual bias at post-treatment is predictive of a return of complaints. Spider fearfuls associated spiders relatively strongly with both harm- and contamination-related outcomes, and these biased expectancies were effectively reduced following treatment. However, as residual bias at post-treatment did not predict return of spider fear at two months and two years followup, it remains unclear whether UCS-expectancies enhance fear of spiders. 
Recent models of specific phobias assume that cognitive biases play an important role in the genesis and maintenance of these phobias (Davey, 1997). One such bias is the UCS-expectancy bias, which can be defined as the tendency to over-associate fear-relevant conditioned stimuli (CS) with the occurrence of aversive unconditioned stimuli (UCS) (e.g., Davey, 1992; Tomarken, Mineka, \& Cook, 1989). Previous work demonstrated that spider fearful undergraduates are indeed characterized by UCS-expectancy biases for aversive outcomes to follow a confrontation with spiders, whereas non-spider fearful individuals do generally not display such biases (Cavanagh \& Davey, 2000; van Overveld, de Jong, \& Peters, 2006). Additionally, although differential UCS-expectancies exist for various animal types (Cavanagh, Davey, \& Lamb, 2003; van Overveld et al., 2006), only expectancy biases for phobia-relevant animals (i.e., spiders) were significantly more pronounced in spider fearful individuals (van Overveld et al., 2006). Thus, UCS-expectancies only appear inflated for phobia-relevant stimuli. Moreover, spider fearful individuals do not only markedly overestimate UCS-expectancies of a harmful outcome following a hypothetical confrontation with a spider in comparison to nonfearfuls, they also consistently underestimate the probability of a harmful outcome following other, fear-irrelevant stimuli (Cavanagh \& Davey, 2000). Such cognitive biases may maintain fear of spiders by inducing behavioral avoidance towards fear-relevant stimuli (e.g., Whittal \& Goetsch, 1997; Jones \& Menzies, 2000), thereby reducing the opportunity for the extinction of fear. Moreover, avoidance of the CS (i.e., spiders) will impede the correction of UCS-expectancy biases (Davey, 1997).

There is increasing evidence that in addition to harm-related UCS-expectancies, contamination-related expectancies are also involved in spider fear. Spider fearful individuals are characterized by disgust and contamination-related associations with regard to spiders on explicit (de Jong \& Muris, 2002; van Overveld et al., 2006) as well as implicit measures (Huijding \& de Jong, 2007). Furthermore, spider fearful individuals do not only display an UCSexpectancy bias for harm-related consequences, but also display a UCS-expectancy bias for contamination-related consequences to follow a confrontation with spiders (van Overveld, de Jong, \& Peters, 2006). Underlining the relative importance of contamination-related UCSexpectancies in spider distress, previous research already showed that contamination-related UCS-expectancy bias was the single best predictor of phobic avoidance during a behavioral approach test (de Jong \& Peters, 2007).

The present study was designed to further investigate the role of contamination-related UCS-expectancy bias in the maintenance of phobic complaints. As an important first step, the present study sought to replicate the earlier findings in a sample of treatment seeking (rather than analogue) spider distressed individuals. As a second step, the present study tested if contamination-related UCS-expectancies disappear following successful treatment. If indeed contamination-related UCS-expectancy bias plays a critical role in the maintenance of spider distress, the bias should be substantially reduced after successful treatment. Third, a critical implication of the proposed reciprocal relationship between complaints and expectancy bias would be that successfully treated patients who nevertheless display a post-treatment bias are more vulnerable to a return of complaints than patients who do not display such a posttreatment bias (cf. de Jong et al., 1995). Therefore, we also tested the prediction that residual 


\section{CHAPTER 11}

UCS-expectancy biases following successful treatment are predictive of the return of phobic complaints at two-months and two-years follow-up. Additionally, it was studied whether residual contamination- and harm-related UCS-expectancies differ in prognostic value for the return of spider fear.

Thus, UCS-expectancy biases were examined in a treatment-seeking sample of spider fearful individuals and in a group of non-spider fearful control participants. If UCS-expectancy biases indeed contribute to spider fear, then, 1) such UCS-expectancy biases should be significantly stronger in the treatment-seeking sample compared to the control group (replicating earlier results in analogue groups, e.g., van Overveld et al., 2006), 2) the UCS-expectancy biases should be effectively reduced following treatment (cf. de Jong et al., 1992), and, 3) residual bias following successful treatment should be associated with a return of phobic symptoms (de Jong et al., 1995). Since previous research provided evidence indicating that contaminationrather than harm-related expectancies differentiate best between spider fearful and non-spider fearful groups (de Jong \& Peters, 2007; van Overveld et al., 2006), it was anticipated that residual UCS-expectancies for contamination-related outcomes would be most strongly associated with the return of spider fear. To test whether enhanced expectancy biases in high spider fearful individuals are confined to spiders or, instead, represent a more generally enhanced harm- and/or contamination-related UCS expectancy bias, two additional categories of fear-relevant animals were included in the design: A category of low-predation contamination-relevant animals (maggots) and a category of high-predation harm/pain-relevant animals (pit bull terriers) (see also Davey et al., 2003; van Overveld et al., 2006).

\section{Methods}

\section{Participants}

A treatment-seeking sample of high spider fearful individuals $(n=60)$ and an explicitly nonfearful control group $(n=30)$ were recruited through advertisements in local media, and posters. To establish if participants were indeed high or low spider fearful, the Spider Phobia Questionnaire (SPQ; Klorman, Weerts, Melamed, \& Lang, 1974) was used. High spider fearful participants also completed the Symptom Check List (SCL-90; Derogatis, 1977) to ensure that no comorbid disorders were present. The high fearful and non-fearful groups were comparable in sex, age, and educational level. The participants were predominantly female in both groups ( $81.7 \%$ and $80 \%$, respectively). This skewed gender distribution is consistent with the finding that spider phobia is more prevalent amongst women (Fredrikson et al., 1996). Further, of the high spider fearful individuals, $12.7 \%$ completed only primary education, $45.5 \%$ completed secondary education and $41.8 \%$ completed tertiary educational levels. The high spider fearful group had a mean age of 34 years $(S D=11.1$; range 19-77). Of the low spider fearful group, $3.6 \%$ completed primary education, $53.6 \%$ completed secondary education, and $42.9 \%$ obtained tertiary educational levels. The low spider fearful group had a mean age of 35.2 years $(S D=13.1$; range 18-58). 


\section{Materials}

Spider Phobia Questionnaire. The SPQ examines an individuals' fear of spiders. Participants rate their agreement with 31 items on a dichotomous scale (yes $=1 /$ no $=0$ ) (e.g., "I dislike looking at pictures of spiders in a magazine."). Thus, a sum score ranging between 0 and 31 is obtained. Higher scores reflect higher levels of spider fear. The SPQ is internally consistent with Cronbach's alphas between .83 and .91 (Klorman et al., 1974; Muris \& Merckelbach, 1996).

UCS-expectancy questionnaire. This questionnaire assesses participants' expectancies that an aversive consequence will occur following a hypothetical confrontation with a specific animal. The UCS-expectancy questionnaire is similar to the questionnaire used in previous research in an analogue group (van Overveld et al., 2006) and is based on the procedure originally designed by McNally and Heatherton (1993). Before completing the questionnaire, participants receive a verbal introduction, in which they are asked to imagine as vividly as possible that they are about to participate in an experiment. It is explained that during this hypothetical experiment, they will be shown a series of slides depicting several animal types (i.e., dogs, rabbits, maggots, and spiders). Furthermore, participants are told that after each slide, one of three possible consequences will occur: an electrical shock is administered, they will have to drink a sip of a disgusting juice, or nothing happens. After this verbal introduction, participants are asked how many of the slides depicting a certain animal type (i.e., dogs, rabbits, maggots, and spiders) they believe will be followed by one of the before mentioned consequences. Participants rate their expectancies on a 100-mm VAS, ranging from 0 ('none at all') to 100 ('all'). A total of 12 UCS-expectancy judgments were obtained for each participant (4 animals $\mathrm{x}$ 3 consequences). The sequence of the UCS-ratings is fixed in a random order (for a more elaborate description of the procedure, see van Overveld et al., 2006, as well as the verbal introduction in the Appendix). As we were interested in the specific UCS-expectancies for each animal type, UCS-expectancies were calculated by subtracting the expectancy of a neutral outcome from the outcome of interest (for example, Contamination-expectancy for spiders = UCS-expectancy of a sip of juice following spiders - UCS-expectancy of the neutral consequence following spiders).

To control for the influence of undesirable test-retest effects that might undermine the sensitivity of the present design for testing the influence of treatment on UCS expectancy bias, one group of high fearful participants $(n=30)$ only completed the UCS questionnaire after treatment, whereas a second group of high fearful participants $(n=30)$ were tested before treatment as well as after treatment (cf. de Jong et al., 1992).

Behavioral Approach Task (BAT). The BAT is a widely used instrument to assess the degree of avoidance for specific stimuli such as spiders (Arntz, Lavy, van den Berg, \& Rijsoort, 1993; de Jong, Vorage, \& van den Hout, 2000). Here, participants had to approach a medium-sized common house spider (Tegenaria atrica) in eight steps, ranging from looking at a spider in a closed jar, to having a spider walk on their hands. The steps increased in difficulty and participants were informed that they could stop at any point. After each step, they completed two VAS-scales (from 0-100) to assess peak anxiety and peak disgust during the last step. 


\section{CHAPTER 11}

The participants' score was then calculated by summing the amount of actual steps taken on the BAT (range: 0-8). Thus, high BAT-scores represent low avoidance of spiders.

Treatment. All spider fearful individuals received a 2.5 hours, single-session exposure in vivo treatment (see also Öst, 1989). During this procedure, participants were intensively exposed to spiders, had to approach spiders actively, and remained in contact with them until their fear would fade away. This treatment is one of the most effective treatments available in reducing fear of spiders (Öst, 1989). The treatment was conducted by five female students at the university of Groningen, who successfully passed a training course on how to administer this particular type of therapy and followed a Dutch protocol (de Jong \& Keijsers, 1999) based on the work of Öst (1989).

\section{Procedure}

The treatment seeking participants were randomly assigned to one of two groups. Participants in the "after treatment" group first underwent a 2.5 hour exposure in vivo treatment before they participated in the experimental procedure. Participants in the "before treatment" group participated in the experimental procedure before they received treatment. To allow testing of the influence of repeated assessment (test retest effects) the latter group also participated in the experiment after treatment.

On arrival at the lab, all participants completed the SPQ. Only the "before treatment" group and the low fearful control participants also completed the UCS-expectancy questionnaire at this point. After this, all participants conducted a Behavioral Approach Task (BAT) with a real-life medium sized common house spider. For the low fearful group, the data acquisition for the present experiment ended here. However, this research was part of a larger study, and all participants were administered a number of computer tasks in addition to the measurements currently presented (see also Huijding \& de Jong, 2007). High spider fearful individuals $(n=60)$ then received a one-session exposure in vivo treatment. After the treatment session, both fearful groups received the UCS-questionnaire and completed a second BAT. Next, all treated individuals were asked to participate again after a two-months follow-up period. A total of 41 participants agreed to return to the lab and completed all questionnaires and another BAT. After two years, all treated individuals were approached once more, and were asked to complete the SPQ. Two persons refused to complete the questionnaires because they experienced a return of fear, one person could no longer be reached as she moved abroad, and 18 people did not respond even after repeated prompting. Eventually, 39 participants completed the questionnaires at two-years follow up. However, some patients who dropped out at two months follow-up were willing to complete the questionnaires at two-years follow-up and vice versa. Consequently, 29 people of the high spider fearful group participated in all three measurements (post-treatment, two-months, and two-years follow-up). A schematic overview of the study is represented in Figure 1. 


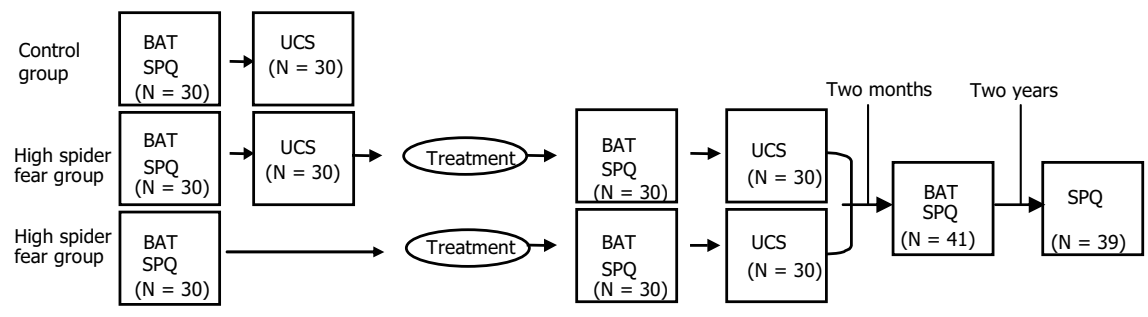

Figure 1. Schematic diagram of the study design.

\section{Results}

\section{Descriptive statistics}

Table 1 depicts the mean scores of the high and low spider fearful groups on all questionnaires. A one-way ANOVA confirmed that high spider fearful individuals were characterized by significantly higher levels of spider fear compared to low spider fearful individuals, as measured by the SPQ $\left(F(1,87)=430.38, p<.01, \eta^{2}=.83\right)$. Additionally, a series of independent samples $\mathrm{t}$-tests showed that at the first measurement at the start of the experiment, no systematic differences were revealed in SPQ-scores, BAT performance, and peak fear / disgust during the BAT between the high spider fearful participants $(n=29)$ who completed all measurements and those who (at some point) dropped out (all $p^{\prime} s>.49$ ).

Further, the number of BAT-steps taken on the BAT prior to treatment was significantly lower in the high spider fearful group compared to the low spider fearful group $(\neg 1,89)=$ $87.71, p<.01, \eta^{2}=.50$ ). In addition, the spider fearful group reported to experience significantly higher levels of peak fear $\left.(\digamma 1,89)=195.95, p<.01, \eta^{2}=.69\right)$ and peak disgust $\left.(F 1,89)=49.38, p<.01, \eta^{2}=.36\right)$ during the last step of the BAT.

Next, a series of paired sample $t$-tests were used to compare post-treatment measurements with pre-treatment measurements. To correct for familywise error, Bonferroni corrected alphas were set to .0125 . These revealed that, following treatment, spider fearful individuals displayed a significant decrease in self-reported spider phobic complaints (SPQ) $(t(59)=10.05, p<.01$, $\left.\eta^{2}=1.29\right)$, as well as an increase in the number of BAT-steps taken $(t(58)=-12.25, p<.01$, $\left.\eta^{2}=1.52\right)$, and a significantly decreased level of peak disgust $\left(t(57)=6.90, p<.01, \eta^{2}=.87\right)$ and peak fear $\left(t(58)=6.35, p<.01, \eta^{2}=1.08\right)$ during the BATs.

Then, a series of paired sample $t$-tests were conducted to compare post-treatment scores with scores at two-months follow-up. Using Bonferroni corrections, alpha was set at .0125. This revealed no significant differences on the SPQ $\left(t(41)=.03, p=.97, \eta^{2}<.01\right)$, indicating that spider fearful individuals generally did not display a return of spider fear at two months followup. However, a significant decrease was observed with regard to the number of BAT-steps taken at two-months follow-up $\left(t(39)=3.63, p<.01, \eta^{2}=.28\right)$ compared to the posttreatment measurements, whereas no significant differences were observed in experienced 


\section{CHAPTER 11}

peak disgust $\left(t(38)=-2.06, p=.05, \eta^{2}=.25\right)$ and peak fear $\left(t(39)=-2.47, p=.02, \eta^{2}=.35\right)$ during that BAT.

For the two-years follow-up measurement, Bonferroni corrections yielded a significance level of .025. A paired-sample $t$-test showed that SPQ-scores at the two-years follow-up measurement were significantly lower compared to SPQ-scores at post-treatment measurement $\left(t(38)=2.51, p=.02, \eta^{2}=.32\right)$. Yet, they were similar to SPQ-scores at two-months follow-up $\left(t(28)=2.15, p=.04, \eta^{2}=.33\right)$. Since effect-sizes appear equal, this may simply be due to the small N. Thus, treatment effects were shown to be consolidated in most cases, and overall return of complaints was minimal. So, in general, it can be concluded that treatment was effective and that although a significant return of avoidance behavior (BAT) was noted after two months, the level of post-treatment spider fear remained stable or even improved (SPQ) throughout the two years.

Table 1

Mean scores on the SPQ, mean no. of BAT steps and mean peak fear and disgust during the final BAT-step for high and low spider fearful groups at pre-treatment, post-treatment, and follow-up measurements.

\begin{tabular}{lllcll}
\hline & Low fear & High fear & & & \\
& Pre & Pre & Post & Follow-Up & Follow-up \\
\hline Indices & & & & Two-months & \multicolumn{1}{l}{ Two-years } \\
SPQ & $2.40(3.59)$ & $20.83(4.15)$ & $14.00(6.25)$ & $14.24(7.11)$ & $11.26(7.29)$ \\
BAT-steps & $7.77(.92)$ & $3.85(2.13)$ & $6.90(1.84)$ & $6.38(2.05)$ & - \\
Peak fear & $8.33(14.52)$ & $77.88(25.15)$ & $45.51(33.91)$ & $58.60(34.89)$ & - \\
Peak disgust & $4.17(13.46)$ & $55.17(38.50)$ & $25.26(30.9)$ & $32.35(37.00)$ & - \\
\hline Valid $\mathrm{n}$ & 30 & 60 & 60 & 41 & 39
\end{tabular}

Note. Standard deviations are described between parentheses

UCS-expectations for maggots, dogs, rabbits.

A 3 (Slide Type: rabbits, maggots, dogs) x 2 (Outcome: shock minus nothing, juice minus nothing) $\times 2$ (Group: low spider fearful, high spider fearful) ANOVA was used to determine if earlier findings on UCS-expectancies could be replicated. For this analysis, only the control and "before treatment" groups were included. The analysis showed that the various classes of animals were differentially associated with specific outcomes (Animal x Outcome: $f(2,55)=$ 13.63, $p<.01, \eta^{2}=.33$ ). Although the low spider fear group had significantly higher expectations of both types of aversive outcomes (Group: $f(1,56)=14.20, p<.01, \eta^{2}=.20$ ) than the high spider fear group, the pattern of expectancies was similar for both groups (Animal x Outcome x Group: $f(2,55)=.33, p=.72, \eta^{2}=.01$ ).

Next, contrasts were obtained and revealed that rabbits were most strongly associated with nothing (nothing compared to shock: $f(1,59)=171.87, p<.001, \eta^{2}=.74$; nothing compared to juice: $\left.f(1,59)=177.71, p<.001, \eta^{2}=.75\right)$. Maggots were associated most strongly with juice (juice compared to nothing: $f(1,57)=12.43, p<.001, \eta^{2}=.18$; juice compared to shock: $\left.f(1,57)=30.83, p<.001, \eta^{2}=.35\right)$. Dogs were associated equally strongly with shock and with nothing (shock compared to nothing: $f(1,58)=.04, p=.84, \eta^{2}<.01$ ), whilst 
associations with juice were significantly lower (juice versus nothing: $f(1,58)=5.75, p=.02$, $\eta^{2}=.09$; juice compared to shock: $f(1,58)=14.37, p<.001, \eta^{2}=.20$ ) (see also Figure 2 ).

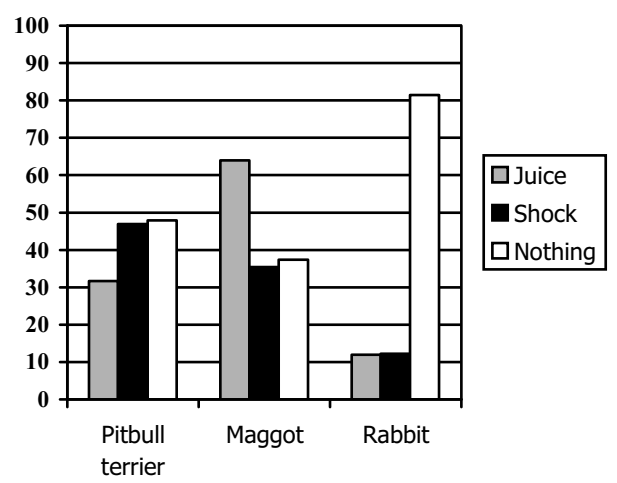

Figure 2. UCS expectancies for different animal types in high $(n=30)$ and low spider fearful individuals $(n=30)$

\section{UCS-expectancies for spiders}

A 2 (Outcome: shock minus nothing, juice minus nothing) x 2 (Group: high and low spider fearful) ANOVA compared the UCS-expectancies for spiders between the "before treatment" and the control group. The intercept was significant which revealed that participants generally expected an aversive outcome following spiders rather than nothing to occur $(f(1,57)=10.70$, $\left.p<.01, \eta^{2}=.16\right)$. However, a significant group effect indicated that this tendency was particularly more pronounced in high spider fearful individuals $\left(f(1,58)=17.23, p<.01, \eta^{2}=\right.$ .23). Figure 3 shows that only high spider fearful participants expected an aversive outcome more strongly after the spider, and that this was similar for both outcome types (Outcome $\mathrm{x}$ Group effect: $\left.f(1,57)=.87, p=.35, \eta^{2}=.02\right)$.

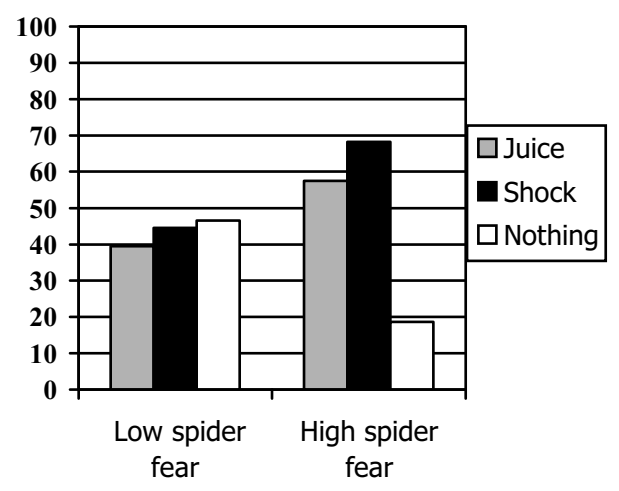

Figure 3. UCS expectancies for spiders in high $(n=30)$ and low $(n=30)$ spider fearful participants 


\section{CHAPTER 11}

\section{UCS-expectancies for spiders following treatment}

To determine whether exposure in vivo treatment effectively reduced UCS-expectancies for spiders, a 2 (Outcome: shock minus nothing, juice minus nothing) x 2 (Group: "before treatment" group, "after treatment" group) ANOVA was used. Thus, pre-treatment UCSexpectancies (shock minus nothing, juice minus nothing) for spiders in the "before treatment" group were compared to the post-treatment UCS-expectancies for spiders of the "after treatment" groupt††. The post-treatment UCS expectancies for spiders of the "before treatment group" were thus excluded in the current analysis. The main effect of Group was significant $\left(F(1,58)=11.82, p<.01, \eta^{2}=.17\right)$, indicating that the UCS-expectancies differed between pre- and post-measurements. The reduction of UCS-expectancies was similar for both types of outcomes as was evidenced by the absence of a significant Outcome $x$ Group interaction $\left(f(1,58)=.15, p=.70, \eta^{2}<.01\right)$. Figure 4 displays a visual summary $\neq \neq \neq$.

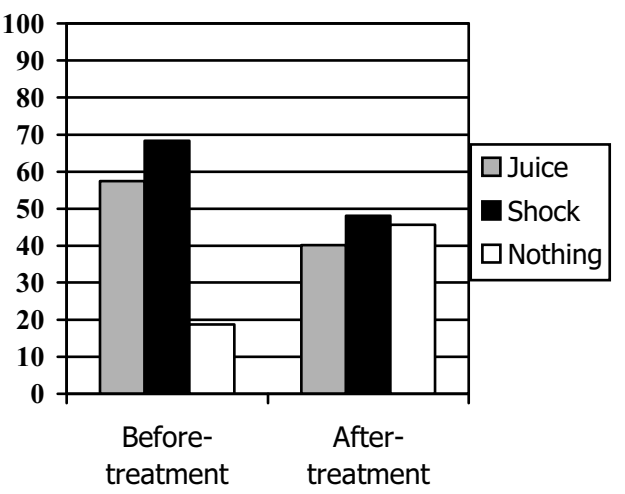

Figure 4. UCS expectancies for spiders for spider fearful participants with either a pre- $(n=30)$ or only posttreatment measurement $(n=30)$

††† The research was designed to prevent possible carry-over effects. To determine whether any carry-over effects were present, a 2 (Outcome: shock minus nothing, juice minus nothing) x 2 (Group : "Before treatment" group, "After treatment" group) ANOVA was conducted on post-treatment UCS-expectancies for spiders. The "before treatment" group completed the UCS-expectancy questionnaire twice, while the "after treatment" group completed the UCS-expectancy questionnaire at post-treatment for the first time. At the post-treatment measurement, no differences were observed in UCS-expectancies for spiders between the two groups $\left(f(1,57)=.83, p=.37, \eta^{2}=\right.$ .01). Thus, there was no evidence of carry-over effects in the phobic group that completed the UCS-questionnaire both before and after treatment.

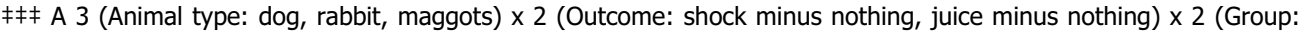
"Before treatment" group, "After treatment" group) ANOVA was conducted to determine whether carry-over effects could be observed for the various animal types. Again, pre-treatment UCS-expectancies for the various animals of the "before treatment" group were compared with only the post-treatment UCS-expectancies of the "after treatment" group. Most importantly for the present context, there was no main effect of Group $\left(f(1,58)=.42, p=.52, \eta^{2}=\right.$ .01 ), indicating that there were no differences in UCS-expectancies between the group with or without a pretreatment measurement. Thus, carry-over effects were not found for the various animal types. 
Additionally, a 2 (Outcome: shock minus nothing, juice minus nothing) x 2 (Time : pretreatment, post-treatment) ANOVA in the "before treatment" group was conducted to examine the decrease in UCS-expectancies for spiders in individuals with a pre- and post-treatment assessment. A similar pattern of a reduction in the UCS-expectancies of both outcome types following spiders was observed after treatment $\left(\Re(1,28)=.82, p=.37, \eta^{2}=.03\right)$.

\section{Prediction of return of fear at two months}

To examine whether the presence of residual UCS-expectancy biases for shock and juice after treatment predict the return of spider phobic complaints at two-months follow-up, a multiple regression analysis was performed with return of spider fear (SPQ-score at follow-up minus the SPQ-score at post-treatment measurement) as dependent variable and the UCS-expectancies at post-treatment measurement for spiders (shock minus nothing, juice minus nothing) as predictor variables. Both predictor variables were entered simultaneously in the regression model. The overall model was not significant $\left(f(2,40)=.97, p=.39, R^{2}=.05\right)$. Neither residual expectancy bias for shock $(\beta=.24, t(36)=-.88, p=.48)$ nor for juice $(\beta=-.40, t(36)$ $=.86, p=.24$ ) could predict a return of spider fear at two-months follow-up.

A similar regression analysis was conducted for the difference in BAT-scores (BAT-scores at follow-up minus the BAT-scores at post-treatment). The overall model was not significant $(\neg 2$, $\left.38)=1.50, p=.24, R^{2}=.08\right)$. Neither post-treatment UCS-expectancies for shock $(\beta=-.44$, $t(36)=-1.362, p=.18)$ nor juice $(\beta=.21, t(36)=.66, p=.51)$ significantly predicted return of spider fear, as indexed by a drop in the number of BAT-steps taken at follow-up compared to the BAT at post-treatment measurement.

\section{Prediction of return of fear at two years}

Regression analyses were performed with the difference in SPQ-scores (SPQ-score at two-years follow-up minus the post-treatment SPQ-score) as dependent variable, and the residual biases at post-treatment measurement for aversive outcomes to follow spiders (juice minus nothing, shock minus nothing) as predictors. The overall model was not significant $(f(2,37)=.42, p=$ $\left..67, R^{2}=.02\right)$. Neither residual biases for the $\operatorname{shock}(\beta=-.28, t(36)=-.88, p=.38)$ nor for the juice $(\beta=.28, t(36)=.86, p=.39)$ predicted SPQ-scores at two years follow-up§§§.

$\S \S \S ~ A ~ 2$ (Outcome: shock minus nothing, juice minus nothing) $\times 2$ (Group : high spider fearful group who did or did not participate at two-years follow-up) ANOVA was conducted for spiders, comparing residual UCS-expectancies for spiders at post-treatment measurement between the people who eventually participated at two-years follow-up, versus those who would drop out. Interaction term Outcome $x$ Group was not significant $(f(1,57)=1.21, p=.27$, $\left.\eta^{2}=.02\right)$, so generally no differences in residual UCS-expectancies for spiders were observed between the people who did or did not participate again after two years. So although it is not unlikely that the people who dropped out did display a return of fear after two years follow-up, this return of complaints is apparently not related to enhanced residual biases in this particular group. 


\section{CHAPTER 11}

\section{Discussion}

The main findings of the present study are as follows: i) high spider fearful participants displayed inflated spider-outcome expectancies for both contamination-related and harmrelated outcomes, ii) both types of UCS-expectancies were significantly reduced following treatment, iii) residual post-treatment UCS-expectancy biases were not associated with a return of phobic symptoms.

The primary aim of the current study was to examine whether UCS-expectancy biases would contribute to spider fear. Therefore, we tested whether these UCS-expectancy biases would be more pronounced in the treatment-seeking group than in the explicitly non-spider fearful group, whether these UCS-expectancy biases are reduced following successful treatment, and finally whether residual UCS-expectancy bias following treatment would be associated with a return of spider fear.

Regarding the first step, it can be concluded that, consistent with prior research in analogue groups (Cavanagh \& Davey, 2000; Diamond, Matchett, \& Davey, 1995; Kennedy, Rapee, \& Mazurski, 1997, van Overveld et al., 2006), inflated UCS-expectancies for aversive outcomes as well as an underestimation of nothing to follow spiders was most pronounced in the high spider fearful group. In addition, and in accordance with earlier findings (Davey et al., 2003; van Overveld et al., 2006), the various animal types were selectively associated with different UCSexpectancies. More specifically, participants generally expected either harmful outcomes (shock) or nothing following confrontation with a harm-related animal (growling dog), contaminationrelated outcomes (sip of nauseating, disgusting juice) following confrontation with a disgusting animal (maggots), and no response at all following confrontation with a neutral animal (rabbit). Most important for the present context, the pattern of selective associations for these animal types was similar for high and low spider fearful groups, whereas UCS-expectancies were, as expected, inflated specifically for spiders in the high spider fearful group. Adding to the idea that contamination-related preoccupations are somehow involved in spider fear, this UCSexpectancy bias was not restricted to harm-related outcomes but also evident for contamination-related outcomes (see also van Overveld et al., 2006, and de Jong \& Peters, 2007 for similar findings in analogue groups). Meanwhile, it should be acknowledged that the UCS-expectancies were strongest for the harm-related outcome. Although this may indicate that expectancies of physical harm are more important in spider fear than contamination-related preoccupations (e.g., Arntz et al, 1993; Edwards \& Salkovskis, 2005), the findings are also consistent with the view that spider phobia essentially reflects a fear of physical contact with disgusting animals (de Jong \& Muris, 2002). Future studies using a harm-related outcome that does not involve direct physical contact with the skin (the critical borderline between the self and the environment) are necessary to more definitely differentiate between both possible explanations of the enhanced expectancies for shock following spiders.

With regard to the second step, the data showed that after a one-session exposure in vivo treatment, UCS-expectancies were indeed significantly reduced. The UCS-expectancy biases for both types of aversive outcomes (shock and juice) were significantly lower in high spider fearful participants at post-treatment measurements compared to UCS-expectancies in high spider 
fearful participants who did not yet received treatment. This is in line with earlier studies that showed that cognitive biases can be successfully modified as a result of treatment (e.g., de Jong et al., 1992).

Regarding the third issue, the present findings showed that the return of fear at two-months and two years follow-up, as indexed by an increase on the SPQ at both measurements and a decrease in the number of BAT-steps taken at two-months follow-up, could not be predicted by residual post-treatment UCS-expectancy biases. This contrasts with earlier findings showing that residual cognitive bias predicted the return of phobic complaints at two-years follow-up (de Jong, van den Hout, \& Merckelbach, 1995). However, underlining recent findings on the effectiveness of exposure in vivo in spider phobia (cf. Choy, Fyer, \& Lipsitz, 2007), no significant return of spider fear, as indexed by the SPQ, was observed at two-months follow-up, and treatment effects appeared further consolidated at two-years follow-up. Although there was a significant decrease in number of BAT-steps at two-months follow-up, no differences were observed in peak disgust and peak fear during the BAT. Therefore, it may be argued that treatment effects were still maintained at two months and even after two years follow-up. Thus, return of phobic complaints appeared to have been only minimal (cf. Teachman \& Woody, 2003) thereby undermining the sensitivity of the present study to find evidence for the hypothesized relationship between residual bias and return of fear. Therefore, the present study does not allow firm conclusions with respect to role of (residual) UCS-expectancies in the return of spider fear.

Several critical comments are in order. First, the possibility remains that the spider fearfuls who did not participate in the follow-up studies did experience a return of fear, and may perhaps also be characterized by a significant increase in UCS-expectancies. However, as the spider fearful persons who refused to participate again after two-years were not characterized by a relatively strong residual UCS-expectancy bias immediately following treatment, it appears that the high spider fearful group at two-years follow-up is still representative of the research population. Second, it should be acknowledged that the present thought experiment procedure could have been influenced by demand characteristics. Most importantly, it cannot be ruled out that the results partly reflect participants' guesses of the experimenter's intentions, which may not necessarily correspond with their own personal judgments about spider-outcome expectancies. However, previous research indicates that the findings that are obtained via similar thought experiments are comparable to the results obtained when conducting the actual 'in vivo' covariation experiments (Davey, 1992; Davey \& Dixon, 1996). Even so, it remains to be established whether UCS-thought experiments are indeed valid instruments for measuring cognitive biases. To more definitely settle this issue, it would be important to verify whether similar results emerge when using an actual (illusory correlation) experiment (e.g., de Jong \& Peters, 2007) or when using more implicit measures that are less susceptible to demand, such as the affective Simon test (e.g., de Jong, van den Hout, Rietbroek, \& Huijding, 2003).

Third, to more directly examine the important issue of causal contributions of UCSexpectancy biases, it would be interesting to conduct an experiment in which harm- and/or contamination-related UCS-expectancy biases are induced in non-anxious individuals and to test whether such a manipulation would indeed result in heightened fear responses upon 


\section{CHAPTER 11}

confrontation with spiders (see for such an approach in the context of attentional bias: Mathews \& Mackintosh, 2000; Mathews \& MacLeod, 2002). It should be noted, however, that this type of study might raise ethical concerns: if indeed UCS-expectancy biases play a causal role in the development of anxiety disorders, one should be very careful with such manipulations (remembering Watson's little Albert). An alternative approach, and even more exciting from a clinical stance, would be to examine whether the unlearning of UCS-expectancy biases in fearful individuals will be paralleled by a decrease of phobic complaints (cf. Pauli, Montoya, \& Martz, 2001).

In summary, consistent with the alleged reciprocal relationship between UCS-expectancy bias and phobic complaints, untreated spider fearful individuals were characterized by UCSexpectancy biases specifically for spiders, and these biases were significantly reduced following treatment. However, it remains to be established whether residual contamination and/or harmrelevant UCS-biases at post-treatment measurement are predictive of return of fear later in life. 


\section{Appendix}

\section{Thought experiment}

This brief questionnaire studies how people perceive experimental research. This will be done by studying beliefs about certain animals.

\section{Description of the experiment}

You are about to participate in a thought experiment. This means that we will ask you to imagine as vividly as possible the following situation. You are invited here to participate in an experimental study at the laboratory. You will be asked to sit down in a comfortable chair, and view a series of slides, projected on the large screen in the lab. Two electrodes will be placed on your upper arm and before the experiment starts a level of electrical shock will be selected in consultation with you. This will be done so the level is certainly unpleasant, yet not painful. During the experiment you will receive shocks at certain moments. Also, a catheter will be inserted in your mouth, and taped to your cheek, so at certain moments a fluid can be injected. This fluid will taste very bitter, and is quite nauseating. The fluid is, however, just like the shock, unpleasant yet harmless and without side effects.

During the experiments you will be shown a series of slides of four types of animals: dog, spiders, maggots and rabbits (at this point, some exemplary slides were shown of a growling dog, a spider, maggots and a white rabbit). Each slide will be shown for exactly six seconds and will immediately be followed by one of these three consequences: either you will receive a short but unpleasant electrical shock, or a shot of the nauseating, bitter fluid will be injected into your mouth, or nothing will happen.

Now imagine you are seated in the chair, with the electrodes attached to your right upper arm and the catheter inserted in your mouth. The light is waning and the first slide will soon appear on the screen. You may now fill in the questionnaire. 
Chapter 12

\section{Discussing the dirt road}

'And they came from the road And he wanted the sun Just to call his own And they walked on the dirt'

PJ Harvey 


\section{CHAPTER 12}

In the present chapter, it will be discussed whether the role of disgust can be seen as a dirt road to anxious psychopathology, and in particular for specific phobias. Moreover, an attempt will be made to provide a first road map for this dirt road. To do so, the relation between disgust and psychopathology will be discussed from multiple perspectives, namely by studying self-report indices of disgust propensity and sensitivity, psychophysiogical parameters, behavioral withdrawal tendencies, and cognitive biases. This will be done by addressing the various research questions on the role of disgust propensity as well as disgust sensitivity in specific phobias, whether separate disgust domains (core, A-R) exist and may be involved in specific fears, and whether people with specific fears tend to display enhanced disgust-related cognitive biases.

\section{Enhanced disgust propensity: a risk factor for developing specific phobias?}

Disgust propensity is defined as the individual tendency to respond with the emotion of disgust. If there is a dirt road to psychopathology, then disgust propensity seems a likely candidate for being a risk factor for developing certain specific phobias that may be disgust-related, such as spider and blood phobia. In accordance with this idea, elevated levels of disgust propensity have been observed in spider phobic individuals (Tolin, Lohr, Sawchuk, \& Lee, 1997, Sawchuk, Lohr, Tolin, Lee, \& Kleinknecht, 2000) and blood phobic individuals (Sawchuk et al., 2000; Schienle et al., 2003). Additionally, elevated levels of disgust propensity were found in various other conditions, such as obsessive compulsive disorders (Thorpe, Patel, \& Simonds, 2003), eating disorders (Troop, Murphy, Bramon, \& Treasure, 2000) and sexual dysfunctions (de Jong, van Overveld, Weijmar-Schultz, Peters, \& Buwalda, in press).

It should be noted, however, that the questionnaires that are currently being used to measure disgust propensity display content overlap with indices of psychopathology, such as specific phobias. For example, questionnaire items involving 'dead bodies' are observed in an index for blood phobia (Blood-Injury Phobia Questionnaire; BIQ; Merckelbach, Muris, de Jong \& de Jongh, 1999) as well as in an index for disgust propensity, the Disgust Scale (DS; Haidt et al., 1994). This may artificially inflate correlations between indices of specific phobias and trait disgust propensity. Currently, only one instrument is available to index disgust propensity irrespectively of disgust elicitors, the Disgust Propensity and Sensitivity Scale (DPSS; Cavanagh \& Davey, 2000). Its psychometric properties were, however, not yet established. Therefore, factor analyses were performed in the present dissertation on the DPSS-R and confirmed that disgust propensity could be reliably established using a revised version of the DPSS (Chapter 3). Moreover, recent work confirmed this factor structure in an independent sample (Olatunji et al., 2007).

As noted above, earlier research linked enhanced disgust propensity to specific fears, such as spider and blood phobia (de Jong \& Merckelbach, 1998; Matchett \& Davey, 1991). Accordingly, the present findings sustain this idea and indicated that elevated levels of disgust propensity (as indexed by the DPSS-R) were associated with fear of spiders (Chapters 3 and 6 ) and fear of blood (Chapters 3, 6, 8, and 10). In line with the view that enhanced disgust 
propensity may be a risk factor for various psychopathological complaints, disgust propensity was also associated with obsessive compulsive symptoms (Chapter 6). Again, this is particularly noteworthy since the DPSS-R does not contain item overlap with items on psychopathology indices, thereby avoiding spuriously inflated correlations. Thus, enhanced disgust propensity in people with specific phobias does not appear to be merely related to artificial overlap between indices of disgust and psychopathology.

Further, disgust propensity (as indexed by DPSS-R) predicted the number of steps that participants were willing to complete during a series of disgust-evoking behavioral experiments (Chapter 4). Thus, disgust propensity appeared associated with behavioral avoidance for specific objects. Yet, although predictive validity of disgust propensity for the behavioral experiments was good, disgust propensity (as indexed by the DPSS-R) did not predict physiological responsivity during a disgust-inducing film. Individual differences in disgust propensity did not correlate well with physiological changes in disgust-relevant parameters such as saliva production, power in the high frequency band of heart rate variability, or heart rate frequency (Chapter 4). Nor did disgust propensity correlate with increased startle amplitudes during disgust slides in an experiment using the startle eye blink response paradigm (Chapter 5). Yet, during the film (Chapter 4) and the viewing of the disgust slides (Chapter 5), increases were noted in disgust-specific facial EMG (i.e., m. levator labii) and subjective reports of disgust. So although the experiments successfully evoked disgust, increases in physiological reactivity were not associated with scores on the DPSS-R.

This may simply indicate that implicit measurements such as physiological reactivity and explicit measurements like self-report questionnaires present largely dissociated phenomena. In previous research on the relation between subjectively experienced stress and objectively observed increases in stress-related physiology, similar dissociations have already been reported (e.g., Segerstrom \& Miller, 2004). It is likely that a certain level of discordance exists between implicit, spontaneous behavior and explicit behavior which can be consciously controlled. Thus, it may be interesting to establish other, more suitable indices of disgust propensity who measure disgust propensity at an implicit level (e.g., using tasks like the Extrinsic Affective Simon Task; Huijding \& de Jong, 2007). This way, it could be examined whether correlations can be found between such implicit parameters of disgust propensity and autonomic physiological reactivity.

\section{Disgust sensitivity and specific phobias}

In the etiology of specific phobias, not only disgust propensity, but also disgust sensitivity may be relevant. Disgust sensitivity is defined as the tendency of the individual to rate the experience of disgust as something horrid. Elevated levels of disgust sensitivity may motivate individuals to avoid specific items or situations due to the prospect of experiencing disgust. Yet, despite its clinical relevance, it has rarely been explored in relation to psychopathology. Moreover, current indices do not measure disgust sensitivity. The data from this dissertation show that the DPSS-R can indeed reliably establish two factors, disgust propensity and disgust 


\section{CHAPTER 12}

sensitivity (Chapter 3). Supporting the relevance of differentiating between these constructs, disgust propensity and disgust sensitivity were differentially associated with particular forms of psychopathology (Chapter 3, 6, 7).

Disgust sensitivity proved the single best predictor for blood phobic complaints (Chapter 3), and was associated with symptoms of obsessive compulsive disorders (i.e., cleaning compulsions) (Chapter 6), eating disorders (Chapter 6), and emetophobia (Chapter 7). Both propensity and sensitivity appeared involved in fear of blood (Chapters 3, 6, 8, and 10) and obsessive compulsive disorders (Chapter 6), so these conditions may reflect a combination of both. Disgust propensity may then be associated with a fear of contact with disgusting and contaminating stimuli (see also de Jong \& Muris, 2002), while elevated disgust sensitivity may present a strong tendency to avoid the expected response to those stimuli (i.e., experiencing disgust).

However, disgust propensity and sensitivity also correlated with symptoms of depression, a disorder which was presumed unrelated to disgust. Relatedly, another study also noted that both disgust traits were correlated with claustrophobia and height phobia, for which no rationale exists (Davey \& Bond, 2005). Moreover, previous research already correlated disgust propensity with separation anxiety (Muris, Merckelbach, Schmidt, \& Tierney, 1999), agoraphobia (Muris et al., 2000), and acrophobia (Davey \& Bond, 2005). Interestingly, this is not just characteristic for the DPSS-R, but also holds for the more widely used questionnaires. A possible explanation could be that the indices of disgust propensity and disgust sensitivity share content overlap with some of these questionnaires. For example, it could be that correlations between disgust propensity, disgust sensitivity and scales on neuroticism and depression could be artificially inflated due to item overlap (e.g., items on worrying or experiencing negative emotions). Future studies should examine these peculiarities more closely by including indices of unrelated psychopathologies and adapting scales in such a way that (thematic) item overlap is avoided (e.g., by removing shared items from one of the questionnaires).

Interestingly, scores on disgust sensitivity (indexed by DPSS-R) did not predict physiological reactivity during a disgusting film clip, nor predict performance on a series of behavioral experiments (Chapter 4). Whether this indicates that the predictive validity of the DPSS-R is insufficient, remains an issue for further investigation. If disgust sensitivity is indeed a risk factor for certain conditions, then future research must investigate whether elevated levels of disgust sensitivity have predictive value for disgust-related withdrawal tendencies. Especially, since this may have serious implications for the treatments of disorders where disgust sensitivity is assumed to be predominantly involved. Most importantly, because the current treatment of choice for specific phobias, gradual exposure in vivo, may then not be sufficient. An alternative and perhaps more successful approach could then be to expose phobic participants to physiological manifestations of disgust (e.g., nausea) in order to extinguish disgust-induced avoidance behavior (cf. thought provocation procedures; Clark, 1986).

Further, although the DPSS-R appears a valuable addition to the arsenal of available disgust indices, it remains to be investigated whether the concepts of disgust propensity and disgust sensitivity can be successfully differentiated from related concepts, in particular, anxiety sensitivity (Reiss et al., 1986). Future research should focus on this issue more closely by 
administering the DPSS-R and the Anxiety Sensitivity Index (ASI; Reiss, 1986) as well as various indices on related psychopathologies. This is particularly important as most participants find it hard to distinguish between anxiety and disgust when mild levels are experienced. It is crucial to establish that disgust indeed presents a factor that contributes uniquely to psychopathology and is not merely an epiphenomenon of anxiety. Previous research already investigated disgust propensity (as indexed by the DS) in relation to anxiety sensitivity (as indexed by the ASI) and demonstrated that the correlation between disgust propensity and clinical complaints is independent of anxiety sensitivity (Muris, Merckelbach, Schmidt, \& Tierney, 1999). Nevertheless, similar studies using the DPSS-R have yet to be conducted.

So, according to the studies in this dissertation, disgust propensity and sensitivity can be reliably distinguished using the DPSS-R and appear differentially related to various specific fears. Interestingly, previous research already observed differential relations between propensity for various disgust domains with specific phobias (de Jong \& Merckelbach, 1998).

\section{Core and A-R disgust in specific phobias}

In the current literature, two distinct types of disgust are well-established, namely, core and animal-reminder disgust (A-R). Remarkably, studies on the validity of these two domains are scarce. Furthermore, although the two disgust types are generally accepted, none of the indices presently available measures these particular domains. It has been suggested that clustering several scales of the DS may examine disgust propensity for core and A-R disgust (Olatunji, Sawchuk, de Jong, \& Lohr, 2006). However, the subscales of the DS are not internally consistent (Haidt et al., 1994). Thus, it may not be surprising that the present data showed that a recently revised version of the DS (DS-R; Olatunji et al., 2007) still does not appear a valid and reliable instrument to assess multiple dimensions of disgust (core, A-R) (Chapter 2). Yet, a two-factor distribution measuring core and A-R disgust did correlate meaningfully with relevant indices. So, several adjustments to the DS-R may eventually show that this distinction is indeed useful. Thus, future researchers should either consider making several adjustments to the DS (e.g., continuous scoring format; see also Olatunji et al., 2007) or using an alternative index (e.g., the DES). It is crucial to do so, since differential associations of these disgust domains may be related to specific fears. A propensity for core disgust may be associated with spider phobia, while a propensity for A-R disgust may be associated with blood phobia (de Jong \& Merckelbach, 1998; Olatunji et al., 2006; Sawchuk et al., 2000). The findings from the present dissertation were largely consistent with this idea. A propensity for A-R disgust was associated with fear of blood (Chapter 3, 8). Core disgust propensity (as indexed by the DQ), however, correlated with both fear of spiders and fear of blood (Chapter 3 ).

These differential associations may help explain certain specific symptomatology in specific fears (e.g., fainting in blood phobia; Page, 1994). Therefore, the current dissertation investigated the psychophysiological patterns of reactivity that are associated with core and A-R disgust to determine whether these disgust types could be characterized by distinct physiological patterns. Earlier studies suggested that disgust is associated with parasympathetic 


\section{CHAPTER 12}

activity (Levenson, 1992) and it has been proposed that core disgust was associated with increased saliva production (Boon, 2002), whereas confrontation with A-R disgust stimuli was associated with decreases in heart rate and blood pressure (Page, 2003). The present dissertation partly sustained these findings. During imagery of both core and animal-reminder disgust stimuli, increases were noted in the production of saliva, which is consistent with an increase in activity in the digestive tract (Chapter 8). This most likely indicates an increase in parasympathetic activity (Proctor \& Carpenter, 2007). For the cardiovascular tract, increases were observed in T-wave amplitude which generally can be taken to indicate sympathetic withdrawal (Kline et al., 1998). However, during disgust imagery, no changes were observed in the high frequency power band of heart rate variability, in heart rate, or in mean arterial blood pressure. So, although core and A-R disgust did not evoke parasympathetic activity in cardiovascular parameters, inducing disgust was associated with an increase in digestive parasympathetic parameters. Furthermore, during a core disgust film (Chapter 4), increases in saliva production were paralleled by enhanced power in the HF band and a decrease in heart rate frequency, which suggested parasympathetic activity in cardiovascular parameters.

These inconsistent findings of chapters 4 and 8 indicate that the way how material is presented could profoundly affect psychophysiological responsivity. During imagery, the participants remain in control of how vividly the objects are imagined and can influence to what extent the emotion is actually experienced themselves. This is not the case for film clips, where such a loss of control may intensify the experience of the stimulus. However, this presents a troublesome paradox in disgust research. On the one hand, anxiety and disgust tend to be closely associated and participants generally find it very difficult to distinguish them when they are experienced in mild levels (e.g., emotions that are experienced during imagery procedures). On the other hand, using film clips could confound physiological investigations since an active desire to want to avoid the stimuli presented is most likely to be associated with increases in sympathetic activity (i.e., the fight/flight response). This may overshadow disgust-induced parasympathetic activity. Consequently, this presents a difficult issue in disgust research, for which no clear answer appears readily available.

Based on the present findings, propensity for core and A-R disgust were established as separate constructs with a similar psychophysiological response pattern. A shared response pattern for different types of disgust confirms earlier postulations that all disgust types eventually evolved from core disgust, while it undermines the idea that the two disgust types may explain unique symptomatology (i.e., vasovagal syncope) in specific conditions (i.e., blood phobia). Contrary to our expectations, A-R disgust was not characterized by a specific cardiovascular pattern. Yet, blood fearful individuals did experience both fear and disgust during A-R imagery. Furthermore, high blood fearful individual participants experienced more dizziness and nausea compared to low fearful individuals during both core and A-R imagery (Chapter 8). Thus, although core and A-R disgust did not have distinct physiological responsitivity, they did evoke stronger subjective experiences of dizziness and nausea. Together with the finding that high blood fearful individuals evaluated disgust relatively more negative (higher disgust sensitivity) (Chapter 3), such responsivity could function as a driving force for the existence and maintenance of blood phobia. 
Thus, if the appreciation of disgust is relatively important to the etiology of certain specific phobias, then it could very well be that people with disgust-related specific fears are characterized by elevated levels of disgust-related pre-occupations. So next, we investigated the role of disgust-related pre-occupations.

\section{UCS-expectancies in psychopathology}

Although it is generally accepted that cognitive biases contribute to the existence and maintenance of specific phobias (Davey, 1997), few studies have been conducted on cognitive biases associated with disgust. One such bias may be the UCS-expectancy bias, which can be defined as the tendency to over-estimate catastrophic outcomes to occur upon confrontation with specific stimuli.

Research under laboratory conditions showed that phobic participants were characterized by inflated expectancies for fear-related outcomes (e.g., electrical shocks) to follow the presentation of phobia-relevant stimuli (McNally \& Heatherton, 1993; Wiedemann, Pauli \& Dengler, 2001). Yet, although disgust appears involved in various clinical conditions and thus, disgust-related outcome expectancies could also prove important, most studies on UCSexpectancy bias only included harm- or fear-relevant outcomes in their design. Moreover, previous studies indicated that people hold separate UCS-expectancies for specific objects (Davey, Cavanagh, \& Lamb, 2003). More specifically, in a hypothetical thought experiment, participants associated disgusting yet non-fierce fear-relevant animals (i.e., maggots) with a disgusting outcome (i.e., sip of a nauseating drink), fierce fear-relevant animals (i.e., sharks) with a fear-related outcome (i.e., electrical shock), and neutral animals (i.e., rabbits) with a neutral outcome (i.e., nothing at all).

In this dissertation, it was confirmed that participants hold differential UCS-expectancies for various animal types in a series of hypothetical thought experiments. It was consistently shown that disgusting maggots were associated with a disgusting outcome (i.e., sip of a nauseating drink), a fear-relevant growling dog with a fear-related outcome (i.e., electrical shock) and a neutral rabbit with no outcome at all in all participants (Chapters 9, 10, 11).

Additionally, high spider fearful individuals (Chapter 9, 11) and high blood fearful individuals (Chapter 10) consistently demonstrated more pronounced aversive UCS-expectancies for their phobic stimulus compared to low fearful individuals. Yet, phobic individuals did not display stronger disgust-expectancies in general, but only for specific phobia-relevant stimuli. This strengthens the idea that enhanced UCS-expectancy biases may be involved in the maintenance of specific fears.

Yet, no evidence emerged to suggest that UCS-expectancy biases may exert a causal influence on specific fears. Contrary to earlier findings that residual cognitive bias predicted the return of phobic complaints at two-years follow-up (de Jong, van den Hout, \& Merckelbach, 1995), the data showed that residual UCS-expectancy biases at post-treatment measurements were not associated with a return of complaints at two-years follow-up (Chapter 11). Thus, it remains to be seen whether UCS-expectancies have a causal role in developing 


\section{CHAPTER 12}

psychopathological complaints. So, further studies are needed to examine whether UCSexpectancy biases contribute causally to specific clinical disorders or that they are merely an epiphenomenon of clinical complaints. Additionally, it may be that disgust- and fear-related expectancy biases contribute differentially to various conditions. Previous research already noted that some specific phobias may best be understood in terms of contamination fear (such as spider phobia; de Jong \& Muris, 2002). This could indicate that both harm- and disgustrelated expectancy biases could be important in the etiology of disgust-related conditions, whereas disgust-related expectancy biases may not be involved in conditions where fear appears predominantly involved (e.g., height phobia).

An interesting alternative approach could be to induce harm- and / or disgust-related UCSexpectancies in the laboratory and test whether this would coincide with increases in fear for certain stimuli. Given the practical and ethical concerns, however, another approach may be to reduce specific UCS-expectancies and examine whether this coincides with a reduction in fear (cf. MacLeod, Rutherford, Campbell, Ebsworthy, \& Holker, 2002). If UCS-expectancy biases are indeed crucially involved in various types of psychopathology, then it could be that reducing disgust-related UCS-expectancies in psychopathologies in which disgust is presumed to be crucially involved results in a reduction of complaints (e.g., fear of blood, washing compulsions in OCD, eating disorders, and emetophobia).

Interestingly, in earlier work, blood phobic participants showed an a priori UCS-expectancy bias for both disgust- and fear-related outcomes to follow hypothetical presentation of blood slides. This bias vanished when participants engaged in the actual experiment and discovered that the combination of the presentation of blood slides and the occurrence of aversive outcomes was completely random (de Jong \& Peters, 2007a). Perhaps similar training sessions can be constructed to teach phobic individuals that although certain aversive outcomes may occur following a confrontation with phobic stimuli, these outcomes may not appear consistently and are not as catastrophic as participants imagine them to be.

Finally, the present dissertation focused mainly on UCS-expectancies using the thought experiment paradigm (see also McNally \& Heatherton, 1993). Future studies should consider exposing high and low fearful individuals to actual illusory correlation (IC) experiments (e.g., de Jong \& Peters, 2007b). This is important to establish whether UCS expectancy biases are robust phenomena that can be readily corrected when disconfirming information is presented. Moreover, such a procedure would allow exploring whether disgust and fear-related outcome expectancies vary with respect to their sensitivity to corrective experiences. Such relative (in)sensitivity to disconfirming information may provide important clues with respect to the importance of disgust- and fear-relevant UCS representations to the etiology of certain disorders. 


\section{Closing remarks}

In conclusion, the findings from the present dissertation showed that the most widely used index of disgust propensity (DS-R) may not be psychometrically sound. The DPSS-R proved a suitable alternative to measure disgust propensity. Additionally, this questionnaire is the only scale that also covers disgust sensitivity. Further, the first psychometric evaluations of the DPSS-R were presented by using factor analyses and assessing the DPSS-R in relation to actual disgust behavior and psychophysiological reactivity. Next, the studies from this dissertation provide a first empirical exploration whether disgust propensity and sensitivity are differentially associated with various disorders.

Additionally, previous findings that found separate disgust domains to be associated with distinct disorders (e.g., core disgust in spider phobia, and A-R disgust in blood phobia) were largely confirmed in this dissertation. Yet, it was also shown that core and A-R disgust may not be measured reliably with the DS-R. This emphasizes the need for a reliable index to measure both disgust types adequately. Further, this dissertation provided one of few studies currently available on physiological reactivity of core and A-R disgust.

In agreement with a dirt road to specific phobias, UCS expectancy biases for both harmand disgust-related consequences following phobic objects were more pronounced in spider and blood fearful participants compared to healthy control participants. Thus, disgust appeared associated with specific phobias on self-report measurements, in behavioral experiments, on physiological parameters and using a cognitive approach. The logical next step would be to determine whether disgusting pre-occupations causally contribute to specific phobias or whether they present mere epiphenomena of these conditions (cf. Davey, Bickerstaffe, \& MacDonald, 2006).

In other words, now that we have discovered a dirt road to specific phobias, it is vital to learn whether it is a one- or a two-way street. 
Summary

'Spare me the suspense'

Interpol 


\section{SUMMARY}

In a sauté pan, heat the olive oil, then sauté the onions and the garlic until they are light brown. Beat 3 eggs in a large bowl, crumbling in the feta cheese. Add parsley, half a teaspoon oregano, black pepper, and 24 medium-sized wolf spiders. Melt 4 teaspoons of butter and preheat the oven to $375^{\circ}$. Lay the phyllo dough on the bottom of the baking pan. The ends of each sheet should extend over the edges of the pan. Brush them with butter. Spread spider-and-spinach mixture evenly over the layer of phyllo dough. Make layers with the phyllo dough. Trim the overhanging edges and bake uncovered in the oven until the dough turns a golden brown, about 35-40 minutes (Gordon, 1998).

Despite its excellent nutritional value, most readers of this recipe for a Spider-akopita will not hurry towards the attic to catch the ingredients. Contrary to various non-western countries (Menzel \& D'Aluisio, 1998), spiders are generally not accepted as food in the Netherlands. Most Dutch people will be greatly disgusted at the thought of having to sink their teeth in the body of a spider. Research shows that the mere thought that a spider has been in contact with a perfectly edible object, like a cookie, will render this cookie inedible to most participants (Mulkens et al., 1996). The avoidance of these cookies (or the Spider-akopita) appears associated with feelings of disgust for the spider. Thus, in the present dissertation, it will be investigated whether not only anxiety, but also disgust may be involved in developing certain forms of psychopathology (for example, spider and blood phobia).

In Chapter 1, the theoretical background will be outlined for the current dissertation and it will be confirmed that a confrontation with spiders does indeed not just evoke anxiety, but also strong feelings of disgust in most individuals. Thus, it is possible that disgust is involved in developing certain specific fears. In the literature, it is already known that some forms of psychopathology, such as specific phobias for spiders or blood, are associated with enhanced levels of disgust propensity (de Jong \& Merckelbach, 1998, Matchett \& Davey, 1991, Schienle et al., 2005). Disgust propensity can be defined as a personality trait which determines how readily someone experiences the emotion of disgust. Yet, for the development of psychopathology, another factor may also be relevant, namely disgust sensitivity. This can be defined as the individual tendency to evaluate experiencing disgust as a relatively unpleasant experience. It could well be that only in those people who consider the (physiological) expressions of disgust unpleasant, the chance to develop clinical complaints is increased since they may actively avoid experiencing disgust. So far, this has not been studied. The present dissertation will examine this.

Furthermore, two disgust types are distinguished in the literature: core and animal-reminder disgust (A-R). Core disgust is the disgust that is experienced at the prospect of having to consume certain stimuli (e.g., the Spider-akopita). A-R disgust is the disgust that we experience when confronted with stimuli that remind humans of the similarities between them and members of the animal kingdom (e.g., a rotting skunk cadaver at the side of the road may remind us of our own mortality). It has been speculated in the literature that these two disgust types are differentially related to psychopathology. Core disgust has been proposed to be associated with spider phobia, while A-R disgust has been associated with blood phobia (de 
Jong \& Merckelbach, 1998). In this dissertation, it will be studied whether these two types are indeed differentially related with these conditions, and whether they can be distinguished from each other at a physiological level

Lastly, dysfunctional, irrational expectations can lead to the development of clinical complaints (Davey, 1997). In this dissertation, it will also be investigated whether people with certain specific fears (such as fear of spiders or blood) are characterized by irrational expectancies that a confrontation with the feared object, animal, or situation will be automatically followed by an aversive threat- or disgust-related outcome (like pain or contamination).

Chapter 2 describes a psychometric evaluation of the Disgust Scale-Revised (DS-R; Haidt et al., 1994). This scale assesses disgust propensity for eight specific types of stimuli. Although this questionnaire is the most widely used index in disgust research, its psychometric qualities have rarely been examined. Yet, it is known that the eight subscales are fairly unreliable. A recent validation study showed that clustering the eight subscales to three subscales (measuring Core disgust, A-R disgust, and Contamination) significantly improved its psychometric properties. In the current study, this three-factor distribution was investigated and it did indicate a significant improvement with respect to a one- or two-factor model. However, the results also showed that reliability for the subscales remained low. Although internal consistency improved compared to the original eight-factor distribution, the DS-R is still in need of several adjustments.

Chapter 3 describes a study that involves a new and recently introduced questionnaire, the Disgust Propensity and Sensitivity Scale (DPSS; Cavanagh \& Davey, 2000). One of the problems with indices like the DS is that they measure disgust propensity by enquiring about specific disgust stimuli (such as insects). Thus, indices on psychopathology (e.g., questionnaires on spider phobia) may display content overlap with these indices, which may artificially inflate correlations. The DPSS examines disgust propensity without asking about specific disgust elicitors, which may be an advantage. Further, it is the only questionnaire available that also addresses disgust sensitivity. In the present study, the questionnaire was revised to a shorter version, the DPSS-Revised (DPSS-R). From the results, it was shown that the DPSS-R could reliably distinguish disgust propensity and disgust sensitivity, and that both factors were differentially correlated with psychopathology (spider- and blood phobia).

Chapter 4 also describes a validation study on the DPSS-R. In this study, the predictive validity was examined for disgust propensity and disgust sensitivity. Hereto, it was examined whether higher scores on disgust propensity and disgust sensitivity were correlated with stronger avoidance behavior on a series of (disgusting) behavioral experiments, and whether this is paralleled by stronger psychophysiological disgust-related responsivity during a disgust video. The results showed that disgust propensity but not disgust sensitivity was associated with stronger withdrawal tendencies during the behavioral experiments. Although the video did induce disgust-related physiological activity (i.e., activation of disgust-specific facial muscle activity of the $\mathrm{m}$. levator labii, increases in the HF-frequency of heart rate variability), the two DPSS-R subscales were not associated with physiological activity. The results showed that the 
DPSS-R appears a valid instrument for predicting avoidance behavior, but not physiological responsitivity.

In Chapter 5, it was investigated whether individual differences in disgust propensity and disgust sensitivity are paralleled by differences in the magnitude of the eye-blink reflex. The eye-blink reflex is induced by a sudden, loud noise, but more importantly, is greatly amplified in the presence of anxiogenic stimuli. This amplification is dependent of individual differences in how prone people are to experience fear (Caseras et al., 2006). In this experiment, it was tested whether individual differences in disgust propensity and disgust sensitivity can influence the startle eye-blink response in a similar way. A series of slides was shown to 48 women depicting positive, neutral, anxious, and disgusting stimuli, while during $2 / 3$ of the slides, a 'probe' (loud noise) was presented at unexpected moments. Additionally, facial muscle activity of the $\mathrm{m}$. corrugator (indicator for arousal) and the $\mathrm{m}$. levator labii (indicator for disgust) was measured. It was shown that the startle eye-blink response was not significantly enhanced during anxious and disgusting slides compared to neutral slides. It was concluded that individual differences in disgust propensity and disgust sensitivity were not associated with the induced eye-blink reflex during exposure to disgusting stimuli.

Chapter 6 provides a first exploration whether both factors, disgust propensity and disgust sensitivity, as measured using the DPSS-R, are correlated with various forms of psychopathology. A group of students $(N=216)$ completed the DPSS-R, as well as several questionnaires on presumably disgust-related forms of psychopathology (obsessive compulsive disorder, spider phobia, blood phobia, and eating disorders) and a presumably disgustunrelated disorder (depression), and neuroticism. Disgust propensity was associated with spider phobia, while disgust sensitivity was associated more strongly with obsessive compulsive disorders, and eating disorders. Both were associated with blood phobia. Independent of neuroticism, disgust propensity and disgust sensitivity appeared differentially related to psychopathology.

Chapter 7 involves a first exploration on the relationship between the two disgust traits, disgust propensity and disgust sensitivity, with a relatively under-diagnosed disorder, namely emetophobia (fear of vomiting). Given the strong association with a universal disgust stimulus (vomit) and because participants frequently report to avoid the physiological symptoms that are associated vomit (e.g., nausea), it was hypothesized that disgust sensitivity may be relatively more important in this disorder compared to disgust propensity. Thus, a group of members on a website devoted to emetophobia and which consists of a large sample of emetophobic individuals $(n=172)$, and a control group $(n=39)$ was asked to complete a package of questionnaires via internet. The results indicated that the emetophobic group scored significantly higher on both disgust propensity and disgust sensitivity. In line with our hypotheses, disgust sensitivity predicted emetophobic complaints best.

Chapter 8 addressed the question whether core and A-R disgust are characterized by separate physiological response patterns. It has been proposed in the literature that these disgust types may be differentially associated with psychopathology. More specifically, core disgust is associated with spider phobia, while A-R disgust is associated with blood phobia. (de Jong \& Merckelbach, 1998). If these differential associations are supported by separate 
physiological response patterns, this may help explain typical symptomatolgy in psychopathology (e.g., fainting in blood phobia but not in spider phobia). Both high and low blood fearful individuals were asked to imagine various scripts vividly (core, $A-R$, neutral) while both digestive (saliva production) and cardiovascular ( $T$-wave amplitude, blood pressure, heart rate variability) components of the autonomic nervous system were measured. In line with our expectations, subjective experiences differentiated between high and low blood fearful individuals, although not specifically for the A-R script. Although both disgust scripts were associated with an increase in disgust-induced saliva production, in T-wave amplitude, and activity in facial muscle activity of the $\mathrm{m}$. levator, no differences were observed in physiological reactivity between the core and A-R script. This may indicate that although people distinguish different types of disgust propensity, these types are characterized by a shared physiological response pattern which may function as a mechanism to shield the individual from contamination by hazardous pathogens.

Chapter 9 studies the recent finding that people hold differential expectancies (UCSexpectancies) for the possible consequences that may follow the confrontation with various animal types (Davey et al., 2003). In the current study, it was examined whether similar UCSexpectancies are observed in high spider fearful individuals, and whether they particularly expect disgust-related outcomes to follow a confrontation with a spider. High $(n=27)$ and low ( $n=28$ ) spider fearful individuals engaged in a hypothetical thought experiment, where they were asked to rate the probability that a series of slides depicting spiders, maggots, pit bulls, or rabbits, would be followed by administration by the experimenter of a pain/fear-related electrical shock, a disgust-related sip of a nauseating juice, or nothing. The results indicated that the participants generally associated the maggots with the disgusting juice, the pit bull with the electrical shock, and rabbits with nothing. Spiders were associated with both aversive outcomes, although this was more pronounced in the high spider fearful group. UCSexpectancies for the disgust-related outcome type (nauseating juice) proved the single best predictor for spider phobia. These findings support the idea that disgust is involved in spider phobia.

Chapter $\mathbf{1 0}$ focuses on a study to determine whether blood phobic participants may also be characterized by a tendency to over-estimate the likelihood that an aversive outcome will follow after the presentation of a specific (phobic) object. As disgust has been proposed to be crucially involved in blood phobia (Page, 1994), such an over-estimation of disgust-outcomes may be relevant to the etiology of this condition.

High $(n=30)$ and low $(n=30)$ blood fearful individuals participated in a hypothetical thought experiment, where they were asked to rate the possibility that hypothetical presentation of various slides (i.e., rabbit, maggots, dog, weapon, and a bloody wound) would be followed by the administration by the experimenter of a pain/fear-related electrical shock, a disgust-related sip of a nauseating juice, or nothing. Although in general, both aversive outcome types were associated with hypothetical presentation of blood, the tendency to expect an aversive outcome following blood was more pronounced in high blood fearful individuals. This is consistent with the idea that a bias in UCS-expectancies may also be involved in the etiology of blood phobia. 


\section{SUMMARY}

Chapter 11 describes a study to examine whether a bias in UCS-expectancies may display a reciprocal relationship with spider phobia. Both high $(n=60)$ and low spider anxious individuals ( $n=30$ ) engaged in a hypothetical thought experiment, where they were asked to rate the possibility that hypothetical presentation of various slides (i.e., rabbit, maggots, dog, and a spider) would be followed by the administration by the experimenter of a pain/fear-related electrical shock, a disgust-related sip of a nauseating juice, or nothing. Further, it was investigated whether a) stronger UCS-expectancies would be observed in spider fearful individuals who actively seek treatment for their spider fear compared to low spider fearful individuals, b) whether these UCS-expectancies were reduced by an exposure in vivo treatment, c) whether residual bias following treatment could predict a return of complaints after a twoyears follow-up period.

Spider fearful individuals associated spiders relatively strongly with both fear/ pain- as well as disgust-related outcomes, and these UCS-expectancies were effectively reduced following treatment. Residual bias did not predict a return of spider fear after two years. Since the present study could only partially confirm a reciprocal relationship, further research is needed to establish whether a bias in UCS-expectancies may exert a causal influence on the development of certain specific fears.

Chapter 12 summarizes the main findings of this dissertation. First, it was concluded that the most widely used index currently being used, the Disgust Scale, did not appear a valid and reliable instrument for measuring disgust propensity. The findings from this dissertation showed that the DPSS-R may provide a valid and reliable alternative, which as an additional advantage, is the only instrument to index both disgust propensity and disgust sensitivity. Moreover, these two disgust traits appeared differentially associated with distinct types of psychopathology. Further, disgust propensity for core and A-R disgust were shown to be separate categories of disgust but with a similar physiological response pattern consisting mostly of increases in the parasympathetic branch of the autonomic nervous system (increases in saliva production, HFfrequency of heart rate variability, and reduction in heart rate) and activity in disgust-specific facial musculature (activity in disgust-specific m. levator labii). Lastly, it was shown that both fear- and disgust-related UCS-expectancies were more pronounced in high spider- and bloodfearful individuals compared to low fearful individuals, but only for their phobic object. This bias in UCS-expectancies could be successfully reduced via a one-session exposure in vivo treatment. In addition to these general findings, several recommendations for future research were made. 


\section{Samenvatting}

'I just keep repeating myself, I just keep repeating myself' Super Furry Animals 


\section{SAMENVATTING}

Verwarm olijfolie in een pan, en bak daarin de uien en knoflook snel bruin. Klop 3 eieren in een grote kom, en kruimel de feta-kaas erin. Voeg vervolgens peterselie, een halve theelepel oregano, zwarte peper en 24 medium grote wolfspinnen toe. Smelt 4 eetlepels boters en verwarm ondertussen de oven op 190 . Leg de yufkas (dunne deegvellen) in de braadpan, en zorg dat de rand net over de pan vallt. Besmeer ze met boter. Bedek de yufka gelijkmatig met het spinspinazie mengsel en leg de volgende yufka eroverheen zodat laagjes ontstaan. Snij de rand af en bak het geheel in de oven voor 35/40 minuten tot het deeg goudbruin is (Gordon, 1998).

Ondanks de uitstekende voedingswaarde zal menig lezer van bovenstaand recept voor de spin-akopita toch niet de ingrediënten gaan verzamelen op zolder. Spinnen worden in Nederland, in tegenoverstelling tot menig niet-westers land (Menzel \& D'Aluisio, 1998) niet gezien als acceptabel voedsel. De meeste Nederlanders zullen walgen van de gedachte de tanden in een spinnelijfje te moeten zetten. Sterker nog, onderzoek toont aan dat zelfs de gedachte dat een spin ook maar in contact is geweest met een eetbaar object, bijvoorbeeld een koekje, al voldoende is voor veel mensen om een dergelijk item niet meer te willen nuttigen (Mulkens et al., 1996). De vermijding van het koekje (of een spin-akopita) lijkt geassocieerd met de walging die de spin oproept. In de huidige dissertatie zal dan ook worden onderzocht of walging, naast angst, betrokken zou kunnen zijn bij het ontwikkelen van bepaalde vormen van psychopathologie (bijvoorbeeld spin- en bloedfobie).

In Hoofdstuk 1 wordt het theoretisch kader geschetst van deze dissertatie, en wordt bevestigd dat de confrontatie met spinnen bij menigeen inderdaad niet alleen angst oproept, maar ook sterke gevoelens van walging. Het is dus mogelijk dat walging een rol speelt bij het ontwikkelen van bepaalde specifieke angsten. Uit de literatuur is reeds bekend dat een aantal vormen van psychopathologie, zoals een specifieke fobie voor spinnen of bloed, geassocieerd zijn met hogere niveaus van walgingsgeneigdheid (de Jong \& Merckelbach, 1998, Matchett \& Davey, 1991, Schienle et al., 2005), ofwel, een persoonlijksheidstrek die bepaalt hoe snel iemand walging ervaart. Voor de ontwikkeling van psychopathologie kan echter een andere factor ook van belang zijn, namelijk walgingsgevoeligheid. Dit is de mate waarin het individu het ervaren van walging eigenlijk vervelend vindt. Het zou kunnen dat juist alleen voor die mensen die de (lichamelijke) expressie van walging als negatief ervaren, de kans toeneemt om klinische klachten te ontwikkelen omdat zij actief het ervaren van de emotie walging gaan vermijden. Tot op heden is dit echter niet onderzocht. Vandaar dat dit in deze dissertatie werd bestudeerd.

Eveneens worden in de literatuur twee hoofdcategorieën onderscheiden wat betreft het type stimuli waarvoor mensen walging ervaren: kernwalging en animal-reminder walging (A-R). Kernwalging betreft de walging voor stimuli bij het vooruitzicht deze te moeten nuttigen (bijvoorbeeld de spin-akopita). A-R walging is de walging die we ervaren bij stimuli die de mens confronteren met de overeenkomsten die de mens deelt met dieren (bijvoorbeeld een rottend stinkdierlijkje aan de kant van de weg, dat ons confronteert met de vergankelijkheid van ons eigen leven). $\mathrm{Er}$ is wel gespeculeerd in de literatuur dat deze twee types differentieel met psychopathologie samenhangen. Zo zou kernwalging een sterkere rol spelen bij spinangst 
terwijl A-R walging juist weer bij bloedfobie een grotere rol wordt toegedicht (de Jong \& Merckelbach, 1998). In deze dissertatie zal de vraag worden bestudeerd of deze twee types mogelijk differentieel met verschillende stoornissen samenhangen, en of ze ook van elkaar kunnen worden onderscheiden op fysiologisch niveau.

Tenslotte kunnen disfunctionele, irreëele verwachtingen leiden tot de ontwikkeling van klinische klachten (Davey, 1997). In deze dissertatie is onderzocht of mensen met een bepaalde specifieke angst (zoals angst voor spinnen of bloed) zich kenmerken door de irrationele verwachting dat een confrontatie met het gevreesde object, dier of situatie automatisch gevolgd zal worden door een akelige aan dreiging of walging gerelateerde uitkomst (zoals pijn of besmetting).

Hoofdstuk 2 beschrijft een psychometrische studie naar de Disgust Scale-Revised (DS-R; Haidt et al., 1994). Deze schaal meet walgingsgeneigdheid voor een achttal specifieke types stimuli. Hoewel deze vragenlijst veruit de meestgebruikte vragenlijst is in het onderzoek naar de emotie walging, zijn de psychometrische eigenschappen van de DS-R zelden onderzocht. Desalniettemin is bekend dat de acht subschalen vrij onbetrouwbaar zijn. Een recente validatiestudie toonde aan dat een mogelijke clustering van de acht subschalen tot drie subschalen (Kernwalging, A-R walging, en Contaminatie) een aanzienlijke verbetering zou zijn. In de huidige studie is deze driedeling onderzocht, en blijkt deze inderdaad een significante verbetering ten opzichte van een één of twee-factoren model. De betrouwbaarheid van de subschalen bleef echter laag. Hoewel de betrouwbaarheid van de afzonderlijke subschalen verbetert ten opzichte van de oorspronkelijke achtdeling, laten de resultaten ook zien dat de DS-R op een aantal punten nog verbetering behoeft.

Hoofdstuk 3 beschrijft een studie naar een nieuwe, recent ontwikkelde vragenlijst, de Disgust Propensity and Sensitivity Scale (DPSS; Cavanagh \& Davey, 2000). Eén van de problemen van lijsten als de DS is dat deze walgingsgeneigdheid meten door te vragen naar specifieke walgingsstimuli (bijvoorbeeld insecten). Hierdoor ontstaat er regelmatig inhoudelijke overlap met lijsten naar psychopathologie (bijvoorbeeld vragenlijsten naar spinangst), hetgeen correlaties tussen beide indices kunstmatig kan verhogen. De DPSS onderzoekt walgingsgeneigdheid zonder specifiek naar objecten te vragen, hetgeen een sterk voordeel kan zijn. Voorts is het de enige vragenlijst naar walgingsgevoeligheid. De huidige studie onderzocht de psychometrische eigenschappen van de DPSS door middel van factoranalyse. Op basis van de resultaten is de vragenlijst gereviseerd tot een kortere versie, de DPSS-Revised (DPSS-R). Uit de resultaten bleek dat de DPSS-R walgingsgevoeligheid en walgingsgeneigdheid valide en betrouwbaar meet, en dat beide factoren ook differentieel samenhingen met psychopathologie (spin- en bloedfobie).

Hoofdstuk 4 beschrijft eveneens een validatiestudie naar de DPSS-R. In deze studie werd de predictieve validiteit bestudeerd van zowel walgingsgevoeligheid als walgingsgeneigdheid. Hiertoe is bekeken of hogere scores op walgingsgevoeligheid en walgingsgeneigdheid samengaan met sterker vermijdingsgedrag op een serie (walgelijke) gedragsexperimenten en of er parallel hieraan sterker psychofysiologische walgingsgerelateerde responsiviteit blijkt bij het bekijken van een walgelijke video. Uit de resultaten bleek dat walgingsgeneigdheid, maar niet walgingsgevoeligheid geassocieerd was met het sterker vermijdingsgedrag gedurende de 


\section{SAMENVATTING}

gedragstesten. Hoewel de video wel fysiologische en walgingsgerelateerde activiteit induceerde (meer specifiek, activatie in walgingsspecifieke faciale spieractiviteit van de $\mathrm{m}$. levator labii, toename in de HF-frequentie band van hartslagvariabiliteit), hingen de twee DPSS-R subschalen niet samen met deze fysiologische activatie. Deze resultaten lieten zien dat de DPSS-R een valide instrument is voor het voorspellen van gedrag, maar niet voor fysiologische responsen.

In Hoofdstuk 5 wordt bekeken of individuele verschillen in walgingsgevoeligheid en walgingsgeneigdheid gepaard gaan met verschillen in de sterkte van de oogknipper-reflex. De oogknipper-reflex wordt opgewekt door een onverwacht hard geluid, maar belangrijker is dat de oogknipper-reflex versterkt wordt in aanwezigheid van anxiogene stimuli. Deze versterking is afhankelijk van individuele verschillen in hoe gevoelig mensen zijn voor het ervaren van angst (Caseras et al., 2006). In dit experiment is getest of individuele verschillen in walgingsgevoeligheid en walgingsgeneigdheid op identieke wijze deze oogknipperreflex beïnvloeden. Aan 48 vrouwen werd een serie dia's getoond van positieve, neutrale, angstige, en walgelijke stimuli, waarbij gedurende $2 / 3$ van de dia's op onverwachte momenten een 'probe' (hard geluid) werd gepresenteerd. Eveneens werd faciale spieractiviteit van de $\mathrm{m}$. corrugator (indicator voor arousal) en de $\mathrm{m}$. levator labii (indicator voor walging) gemeten. Hieruit bleek dat de oogknipper-reflex niet sterker was tijdens anxiogene en walgelijke dia's ten opzichte van neutrale dia's. Geconcludeerd werd dat individuele verschillen in walgingsgevoeligheid en walgingsgeneigdheid niet geassocieerd waren met de uitgelokte oogknipper-reflex tijdens blootstelling aan walgelijke stimuli.

Hoofdstuk 6 bespreekt een eerste exploratie of beide factoren, walgingsgevoeligheid en walgingsgeneigdheid, zoals gemeten met de DPSS-R samenhangen met enkele specifieke vormen van psychopathologie. Een groep studenten $(N=216)$ vulde daartoe zowel de DPSS-R in, alsook enkele vragenlijsten naar mogelijk gerelateerde vormen van psychopathologie (obsessief compulsieve stoornis, spinfobie, bloedfobie, en eetstoornissen), een mogelijk ongerelateerde vorm van psychopathologie (depressie), en neuroticisme.

Walgingsgeneigdheid bleek geassocieerd met spinfobie, terwijl walgingsgevoeligheid meer geassocieerd bleek met obsessief compulsieve symptomen en eetstoornis. Beiden waren geassocieerd met bloedfobie. Onafhankelijk van neuroticisme, bleken walgingsgevoeligheid en walgingsgeneigdheid dus differentieel gerelateerd aan psychopathologie.

Hoofdstuk $\mathbf{7}$ behandelt een eerste exploratie naar de relatie tussen beide persoonlijkheidsfactoren, walgingsgevoeligheid en walgingsgeneigdheid, met een relatief ondergediagnosticeerde stoornis, namelijk emetofobie (angst voor overgeven). Vanwege de sterke associatie met een universeel walgelijke stimulus (braaksel) en doordat patiënten juist rapporteren de fysieke symptomen te willen vermijden die deze substantie bij hen opwekt (bijvoorbeeld misselijkheid), zou het kunnen dat juist walgingsgevoeligheid bij deze stoornis een sterkere rol speelt dan walgingsgevoeligheid. Daarom werd een groep leden van een Nederlandse website naar emetofobie $(n=172)$, waar een grote groep patiënten zich heeft verenigd, alsook een controle groep $(n=39)$ gevraagd een pakket vragenlijsten in te vullen op internet. Uit de resultaten bleek dat de emetofobe groep significant hoger scoorde op zowel walgingsgevoeligheid als walgingsgeneigdheid. Conform de verwachtingen bleek juist walgingsgevoeligheid een sterke voorspeller van emetofobe klachten. 
Hoofdstuk 8 gaat in op de vraag of kern- en animal-reminder walging (A-R) mogelijk gekenmerkt worden door een afzonderlijk fysiologisch reactiepatroon. Uit de literatuur is bekend dat deze types walgingsgeneigdheid differentieel zouden kunnen samenhangen met verschillende vormen van psychopathologie. Zo zou kernwalging juist geassocieerd zijn met spinangst terwijl A-R-walging meer geassocieerd zou zijn met bloedfobie (de Jong \& Merckelbach, 1998). Als een dergelijke differentiële associatie wordt ondersteund door afzonderlijke fysiologische patronen, zou dit een verklaring kunnen zijn voor specifieke symptomatologie bij psychopathologie (bijv. flauwvallen onder bloedangstigen maar niet onder spinangstigen).

Zowel hoog als laag-bloedangstige mensen werd gevraagd om enkele scripts actief in te beelden (kern, A-R, neutraal) terwijl onderwijl digestieve (speekselproduktie) alswel cardiovasculaire (T-wave amplitude, bloeddruk, hartslagvariabiliteit) componenten van het autonome zenuwstelsel werden gemeten. Conform de verwachting bleken subjectieve expressies van walging te differentiëren tussen hoog en laag bloed angstigen, maar dit was niet specifiek voor A-R walging. Hoewel beide walgingsscripts samengingen met een walgingsgeïnduceerde verhoging in speekselproduktie, toename in T-wave amplitude, en activiteit in faciale spieractiviteit van de $\mathrm{m}$. levator labii, bleken er geen verschillen in fysiologische reactiviteit tussen het kern- en A-R script. Dit zou er op kunnen wijzen dat hoewel mensen verschillende types walgingsgeneigdheid onderscheiden, deze types gekenmerkt worden door dezelfde onderliggende fysiologische reactiepatronen die als functie hebben het individu te beschermen tegen schadelijke pathogenen.

Hoofdstuk 9 gaat in op een recente bevinding dat mensen verschillende verwachtingen koesteren (UCS-verwachtingen) voor de mogelijke consequenties die volgen op een confrontatie met bepaalde diertypes (Davey et al., 2003). In de huidige studie werd gekeken of dergelijke UCS-verwachtingen eveneens kunnen worden aangetoond onder hoog spinangstige mensen, en of deze in het bijzonder aan walging gerelateerde uitkomsten verwachten tijdens confrontatie met een spin. Hoog $(n=27)$ en laag $(n=28)$ spinangstigen namen daartoe deel aan een kort gedachtenexperiment, waarin hen werd gevraagd om de waarschijnlijkheid in te schatten dat een serie dia's van spinnen, maden, pit bulls, of konijnen zou worden gevolgd door toediening door de onderzoeker van een pijn/letsel-gerelateerde electrische schok, een walgings-gerelateerd misselijkmakend drankje, en niets. Uit de resultaten bleek dat de deelnemers over het algemeen maden associeerden met het misselijkmakende drankje, pit bulls met de electrische schok, en konijnen met niets. Spinnen werden geassocieerd met beide aversieve consequenties, en sterker in de hoog-spin angstige groep. De UCS-verwachting voor de aan walging gerelateerde consequentie (misselijkmakende drankje) bleek de beste voorspeller voor spinangst. Deze bevindingen ondersteunen de idee dat walging betrokken is bij spinangst.

Hoofdstuk 10 richt zich op een studie waarbij werd bekeken of de neiging om de waarschijnlijkheid te overschatten dat een aversieve consequentie volgt na de presentatie van een bepaald (veelal fobisch) object, eveneens zichtbaar is onder mensen met bloedfobie. Omdat bij bloedfobie walging een cruciale rol wordt toebedicht (Page, 1994), zou een dergelijke 


\section{SAMENVATTING}

overschatting van walgings-consequenties van belang kunnen zijn in de etiologie van deze stoornis.

Hoog $(n=30)$ en laag $(n=30)$ bloedangstigen werd hiertoe gevraagd deel te nemen aan een kort gedachtenexperiment, waarbij hen gevraagd werd de waarschijnlijkheid te schatten dat hypothetische presentatie van bepaalde typen dias (konijn, made, hond, wapen, en een bloedige wond) zouden gevolgd door toediening door de onderzoeker van een aan pijn/letselgerelateerde electrische schok, een aan walging-gerelateerd misselijkmakend drankje, en niets. Ofschoon in het algemeen beide types aversieve consequenties werden verwacht na hypothetische presentatie van bloed, was de verwachting dat een aversieve consequentie zou volgen na de bloed-dias sterker onder hoog angstige individuen. Dit is consistent met de idee dat een bias in UCS-verwachtingen ook betrokken kan zijn in de etiologie van bloedfobie.

Hoofdstuk 11 beschrijft een studie waarin wordt bestudeerd of een bias in UCSverwachtingen een reciproke relatie vertoont met spinangst. Zowel hoog $(n=60)$ als laag spinangstigen $(n=30)$ namen deel aan een kort gedachtenexperiment, waarin de waarschijnlijkheid moest worden geschat dat hypothetische presentatie van bepaalde typen dia's (made, hond, konijn, spin) worden gevolgd door toediening van een electrische schok, misselijkmakend drankje of niets. Onderzocht werd verder of a) spinangstige mensen die actief een behandeling zochten voor hun spinangst sterkere UCS-verwachtingen vertoonden dan laag spinangstigen, b) of deze UCS-verwachtingen werden gereduceerd als gevolg van een exposure in vivo behandeling, $c$ ) of residuele bias na de behandeling een voorspeller was voor terugkeer van de klachten na twee jaar follow-up. Spinangstige individuen associeerden spinnen relatief sterk met zowel pijn/letsel- als walgingsgerelateerde consequenties, en deze verwachtingen werden effectief verminderd na behandeling. Residuele bias bleek echter geen voorspeller voor een terugkeer van spinangst na twee jaar. Aangezien de huidige studie een reciproke relatie deels bevestigde, is vervolgonderzoek wenselijk naar de vraag of een bias in UCSverwachtingen een causale relatie heeft met het ontwikkelen van bepaalde specifieke angsten.

Hoofdstuk 12 vat de belangrijkste bevindingen van het proefschrift samen. Allereerst kon worden geconcludeerd dat het belangrijkste meetinstrument uit de literatuur op dit moment, de Disgust Scale, niet het meest valide en betrouwbaar meetinstrument bleek voor het meten van walgingsgeneigdheid. Uit deze dissertatie bleek echter de DPSS-R een valide en betrouwbaar alternatief, met als voordeel dat dit instrument zowel walgingsgevoeligheid als walgingsgeneigdheid meet. Bovendien bleken deze factoren differentieel geassocieerd met verscheidene vormen van psychopathologie. Daarnaast bleken walgingsgeneigdheid voor kernen A-R walging twee afzonderlijke categorieën maar wel gekenmerkt door eenzelfde fysiologisch reactiepatroon, dat vooral bestaat uit een verhoging in parasympathische activiteit (toename in speekselproduktie, HF-frequentie van hartslagvariabiliteit, reductie in hartslag) en activiteit in walgingsspecifieke faciale spieractiviteit ( $\mathrm{m}$. levator labii). Tenslotte bleken zowel angst- als walgings-gerelateerde UCS-verwachtingen sterker aanwezig onder hoog spinangstige mensen en hoog bloed-angstige mensen, in vergelijking tot niet-angstige mensen, maar alleen voor hun fobisch object. Een bias in de UCS-verwachtingen kon succesvol worden gereduceerd door middel van een één-sessie exposure in vivo behandeling. Naast deze algemene bevindingen worden enkele aanbevelingen voor toekomstig onderzoek gedaan. 


\section{References}

'And I talk to the filth and I walk to the door

$I^{\prime} m$ knee deep in myself But I want to get more of that stuff

Of that stuff'

The Jesus and Mary Chain 


\section{REFERENCES}

Achard, A. (2002). Dagboek van een zoeaaf; 1870; een oorlogsdagboek. Aqua Fortis: Tienen.

Alaoui-Ismaïli, O., Robin, O., Rada, H., Dittmar, A., \& Vernet-Maury, E. 1997). Basic emotions evoked by odorants: comparison between autonomic responses and self-evaluation. Physiology \& Behavior, 62, 713-720.

Amin, J. M., \& Lovibond, P. F. (1997). Dissociations between covariation bias and expectancy bias for fear-relevant stimuli. Cognition and Emotion, 11, 273-289.

Angyal, A. (1941). Disgust and related aversions. Journal of Abnormal and Social Psychology, 36, 393-412.

Arntz, A., Lavy, E., van den Berg, G., \& van Rijsoort, S. (1993). Negative beliefs of spider phobics: a psychometric evaluation of the spider phobia beliefs questionnaire. Advances in Behavior Research and Therapy, 15, $257-277$.

Arrindell, W.A., Mulkens, S., Kok, J., \& Vollenbroek, J. (1999). Disgust sensitivity and the sex difference in fears to common indigenous animals. Behaviour Research and Therapy, 37, 273-280.

Balaban, M. T., \& Taussig, H. N. (1994). Salience of fear/threat in the affective modulation of the human startle blink. Biological Psychology, 38, 117-131.

Barron, B. A., \& van Loon, G. R. (1989). Role of sympathoadrenomedullary system in cardiovascular response to stress in rats. Journal of the Autonomic Nervous System, 28, 179-187.

Boon, M. E. (2002). Walging gemeten; een onderzoek naar de differentiële responsiviteit van orale en animalreminder walging en de domeinspecifieke relatie tussen angst en walging. Master thesis, Maastricht University, Faculty of Health Sciences, Maastricht.

Boschen, M. J. (2007). Reconceptualizing emetophobia: A cognitive- behavioral formulation and research agenda. Journal of Anxiety Disorders, 21, 407-419.

Bouman, T. K., \& van Hout, W. J. P. J. (in preparation). A questionnaire for emetophobia.

Bradley, M. M., Cuthbert, B. N., \& Lang, P. J. (1999). Affect and the startle reflex. In: Dawson, M. E., Schell, A. M., \& Bohmelt, A. H. (Eds.), Startle Modification: Implications for Neuroscience, Cognitive Science, and Clinical Science. Cambridge University Press, New York.

Buchanan, T., \& Smith, J. L. (1999). Using the internet for psychological research: personality Ttesting on the World Wide Web. British Journal of Psychology, 90, 125-144.

Caseras, F. X., Fullana, M. A., Riba, J., Barbanoj, M. J., Aluja, A., \& Torrubia, R., (2006). Influence of individual differences in the Behavioral Inhibition System and stimulus content (fear versus blood-disgust) on affective startle reflex modulation. Biological Psychology, 72, 251-256.

Cavanagh, K., \& Davey, G. C. L. (2000). The development of a measure of individual differences in disgust. Paper presented to the British Psychological Society, Winchester, UK.

Cavanagh, K., \& Davey, G. C. L. (2000). UCS expectancy biases in spider phobics: underestimation of aversive consequences following fear-irrelevant stimuli. Behaviour Research and Therapy, 38, 641-651.

Charash, M., \& McKay, D. (2002). Attention bias for disgust. Journal of Anxiety Disorders, 16, 529-541.

Charash, M., McKay, D., \& DiPaolo, N. (2006). Implicit attention bias for disgust. Anxiety, Stress \& Coping, 19, 353364.

Choy, Y., Fyer, J. D., \& Lipsitz, J. D. (2007). Treatment of specific phobias in adults. Clinical Psychology Review, 27, 266 - 286.

Clark, D. M. (1986). A cognitive approach to panic. Behaviour Research and Therapy, 24, 461-470.

Clark, L. A., \& Watson, D. (1995). Constructing validity: basic issues in objective scale development. Psychological Assessment, 7, 309-319.

Connolly, K. M., O'Neill, K., Flessner, C. A., \& Olatunji, B. O. (2006). Fear, disgust, and fainting in blood-injectioninjury fears: a multi-method approach. Anxiety, Stress \& Coping, 19, 409-420.

Cook, E. W., Hawk, L. W., Davis, T. L., \& Stevenson, V. E. (1991). Affective individual differences and startle reflex modulation. Journal of Abnormal Psychology, 100, 5-13.

Corr, P. J., Kumari, V., Wilson, G. D., Checkley, S., \& Gray, J. A. (1997). Harm avoidance and affective modulation of the startle reflex: A replication. Personality and Individual Differences, 22, 591-593.

Darwin, C. R. (1965). The expression of the emotions in man and animals. Chicago: University of Chicago Press, original work published 1872.

Dattilio, F. M. (2003). Emetic exposure and desensitization procedures in the reduction of nausea and a fear of emesis. Clinical Case Studies, 2, 199-210. 
Davey, G. C. L. (1992). An expectancy model of laboratory preparedness effects. Journal of Experimental Psychology, $121,24-40$.

Davey, G. C. L. (1992). Characteristics of individuals with fear of spiders. Anxiety Research, 4, 299-314.

Davey, G. C. L. (1994). The "disgusting" spider: the role of disease and illness in the perpetuation of fear of spiders. Society and Animals, 2, 17-25.

Davey, G. C. L. (1994). Self-reported fears to common indigenous animals in an adult UK population: the role of disgust sensitivity. British Journal of Psychology, 85, 541-544.

Davey, G. C. L. (1997). Phobias; a handbook of theory, research, and treatment. Wiley: Chichester.

Davey, G. C. L. (2003). Doing clinical psychology research: What is interesting isn't always useful. The Psychologist, $16,412-416$.

Davey, G. C. L., Bickerstaffe, S., \& MacDonald, B. (2006). Experienced disgust causes a negative interpretation bias: A causal role for disgust in anxious psychopathology. Behaviour Research \& Therapy, 44, 1375-1384.

Davey, G. C. L., \& Bond, N. (2006). Using controlled comparisons in disgust psychopathology research: The case of disgust, hypochondriasis and health anxiety. Journal of Behavior Therapy and Experimental Psychiatry, 37, 4-15

Davey, G. C. L., Buckland, G., Tantow, B., \& Dallos, R. (1998). Disgust and eating disorders. European Eating Disorders Review, 6, 201-211.

Davey, G. C. L., Cavanagh, K., \& Lamb, A. (2003). Differential aversive outcome expectancies for high- and lowpredation fear-relevant animals. Journal of Behavior Therapy and Experimental Psychiatry, 34, 117-128.

de Jong, P. J. (1996). Walging en spinfobie. Gedragstherapie, 29, 165-179.

de Jong, P., Andrea, H., \& Muris, P. (1997). Spider phobia in children: disgust and fear before and after treatment. Behaviour Research and Therapy, 35, 559-562.

de Jong, P. J., van den Hout, M. A., \& Merckelbach, H. (1995). Covariation bias and the return of fear. Behaviour Research and Therapy, 33, 211-213.

de Jong, P. J., van den Hout, M. A., Rietbroek, H., \& Huijding, J. (2003). Dissociations between implicit and explicit attitudes towards phobic stimuli. Cognition and Emotion, 17, 521-545.

de Jong, P. J., \& Keijsers, G. P. J. (1999). Protocollaire behandeling van patiënten met een specifieke fobie: 1-sessie exposure in vivo. In G. P. J. Keijsers, A. van Minnen, \& C. A. L. Hoogduin (Eds.) Protocollaire behandelingen in de ambulante GGZ: Deel 2. Zeist: Bohn Stafleu Van Loghum (pp.69-99).

de Jong, P., \& Merckelbach, H. (1998). Blood-injection-injury phobia and fear of spiders: domain specific individual differences in disgust sensitivity. Personality and Individual Differences, 24, 153-158.

de Jong, P. J., Merckelbach, H., Arntz, A., \& Nijman, H. (1992). Covariation detection in treated and untreated spider phobics. Journal of Abnormal Psychology, 101, 724-727.

de Jong, P., \& Muris, P. (2002). Spider phobia: interaction of disgust and perceived likelihood of involuntary physical contact. Journal of Anxiety Disorders, 16, 51-65.

de Jong, P. J., van Overveld, M., Weijmar-Schultz, W., Peters, M.L., \& Buwalda, F.M. (in press). Disgust and contamination sensitivity in vaginismus and dyspareunia. Archives of Sexual Behavior.

de Jong, P. J., \& Peters, M. L. (2007a). Blood-injection-injury fears: Harm- vs. disgust-relevant selective outcome associations. Journal of Behavioral Therapy and Experimental Psychiatry, 38, 263-274.

de Jong, P. J., \& Peters, M. L. (2007b). Contamination vs. harm-relevant outcome expectancies and covariation bias in spider phobia. Behaviour Research and Therapy, 45, 1271-1284.

de Jong, P. J., Peters, M. L., \& Vanderhallen, I. (2002). Disgust and disgust sensitivity in spider phobia: facial EMG in response to spider and oral disgust imagery. Journal of Anxiety Disorders, 16, 477-493.

de Jong, P. J., Visser, S., \& Merckelbach, H. (1996). Startle and spider phobia; Unilateral probes and the prediction of treatment effects. Journal of Psychophysiology, 10, 150-160.

de Jong, P. J., Vorage, I., \& van den Hout, M. A. (2000). Counterconditioning in the treatment of spider phobia: Effects on disgust, fear, and valence. Behaviour Research and Therapy, 38, 1055-1069.

Deacon, B. \& Olatunji, B. O. (2007). Specificity of disgust sensitivity in the prediction of behavioral avoidance in contamination fear. Behaviour Research and Therapy, 45, 2110-2120.

Derogatis, L. R. (1977). SCL-90-R; Administration, scoring, and procedures. Baltimore: John Hopkins University.

Diamond, D., Matchett, G., \& Davey, G. C. L. (1995). The effect of prior fear levels on UCS-expectancy ratings to a fear-relevant stimulus. The Quarterly Journal of Experimental Psychology, 48, 237-247. 


\section{REFERENCES}

Druschel, B. A., \& Sherman, M. F. (1999). Disgust sensitivity as a function of the Big Five and gender. Personality and Individual Differences, 26, 739-748.

Dugan, W., McDonald, M. V., Passik, S. D., Rosenfeld, B. D., Theobald, D., \& Edgerton, S. (1998). Use of the Zung Self-Rating Depression Scale in cancer patients: Feasibility as a screening tool. Psycho-Oncology, 7, 483-493.

Edwards, S., \& Salkovskis, P. M. (2006). An experimental demonstration that fear, but not disgust, is associated with return of fear in phobias. Journal of Anxiety Disorders, 20, 58-71.

Egloff, B., \& Schmukle, S. C. (2002). Predictive validity of an implicit association test for assessing anxiety. Journal of Personality and Social Psychology, 83, 1441-1455.

Eysenck, H. J., \& Eysenck, S. B. G. (1975). Manual of the Eysenck Personality Questionnaire. San Diego, CA: Educational and Industrial Testing Service.

Fallon, A. E., \& Rozin, P. (1983). The psychological basis of food rejections by humans. Ecology of Food and Nutrition, 13, 15-26.

Fessler, D. M. T., \& Haley, K. J. (2006). Guarding the perimeter: The outside-inside dichotomy in disgust and bodily experience. Cognition and Emotion, 20, 3-19.

Filion, D. L., Dawson, M. E., \& Schell, A. M. (1998). The psychological significance of human startle eyeblink modification: a review. Biological Psychology, 47, 1-43

Fredrikson, M., Annas, P., Fischer, H., \& Wik, G. (1996). Gender and age differences in the prevalence of specific fears and phobias. Behaviour Research, and Therapy, 34, 33-39.

Fridlund, A. J., \& Cacioppo, J. T. (1986). Guidelines for human electromyographic research. Psychophysiology. 23, 567-598.

Garner, D.M., \& Garfinkel, P. E. (1979). The Eating Attitudes Test: An index of the symptoms of anorexia nervosa. Psychological Medicine, 9, 1-7.

Gawronski, B., LeBel, E. P., \& Peters, K. R. (2007). What do implicit measures tell us? Perspectives on Psychological Science, 2, 181-193.

Gerlach, A. L., Spellmeyer, G., Vögele, C., Huster, R., Stevens, S., Hetzel, G., \& Deckert, J. (2006). Blood-injury phobia with and without a history of fainting: Disgust sensitivity does not explain the fainting response. Psychosomatic Medicine, 68, 331-339.

Gilleard, C. (2007). Old Age in Ancient Greece: Narratives of desire, narratives of disgust. Journal of Aging Studies, 21, 81-92.

Gordon, D. G. (1998). The Eat-A-Bug Cookbook; 33 ways to cook grasshoppers, ants, water bugs, spiders, centipedes, and their kin. Ten Speed Press, Berkeley.

Griffiths, J., \& Troop, N. A. (2006). Disgust, fear and dieting concerns in a non-clinical population. Anxiety, Stress \& Coping, 19, 421-433.

Grillon, C., Ameli, R., Foot, M., \& Davis, M. (1993). Fear-potentiated startle: relationship to the level of state / trait anxiety in healthy subjects. Biological Psychiatry, 33, 566-574.

Guyton, A. C., \& Hall, J. E. (2006). Textbook of medical physiology - $11^{\text {th }}$ edition. Philadelphia, Elsevier Saunders.

Haidt, J., McCauley, C., \& Rozin, P. (1994). Individual differences in sensitivity to disgust: a scale sampling seven domains of disgust elicitors. Personality and Individual Differences, 16, 701-713.

Hamm, A. O., Greenwald, M. K., Bradley, M. M., \& Lang, P. J. (1993). Emotional learning, hedonic change, and the startle probe. Journal of Abnormal Psychology, 102, 453-465.

Hayslett, M. M, \& Wildemuth, B. M. (2004). Pixels or pencils? The relative effectiveness of Web-based versus paper surveys. Library \& Information Science Research, 26, 73-93.

Hiemstra, T. (2007). Differential predictive validity of automatic disgust and harm associations for spontaneous disgust-specific physiological responsivity. Unpublished Master thesis. University of Groningen, the Netherlands.

Himle, J. A., McPhee, K., Cameron, O. G., \& Curtis, G. C. (1989). Simple phobia: evidence for heterogeneity. Psychiatry Research, 28, 25-30.

Hornby, P. J. (2001). Central neurocircuitry associated with emesis. The American Journal of Medicine, 111, 106S112S.

van Hout, W. J. P. J., \& Bouman, T. K. (2006). Clinical features of emetophobia (fear of vomiting): Prevalence and comorbidity. Manuscript submitted for publication. 
van Hout, W. J. P. J., Oude Lansink, P., \& Bouman, T. K. (2005). De fenomenologie en comorbiditeit van emetofobie (angst voor overgeven). Gedragstherapie, 38, 49-64.

Hu, L.-T., \& Bentler, P. M. (1999). Cut-off criteria for fit indexes in covariance structure analysis: Conventional criteria versus new alternatives. Structural Equation Modeling, 6, 1-55.

Huijding, J. (2006). Automatic affective associations and psychopathology. (Doctoral dissertation, University of Groningen).

Huijding, J., \& de Jong, P.J. (2006). Specific predictive power of automatic spider-related affective associations for controllable and uncontrollable fear responses toward spiders. Behaviour Research and Therapy, 44, 161-176.

Huijding, J., \& de Jong, P. J. (2007). Beyond fear and disgust: the role of specific harm and contamination-related associations in spider phobia. Journal of Behavior Therapy and Experimental Psychiatry, 38, 200-211.

Huijding, J., \& de Jong, P. J. (in press). Implicit and explicit attitudes towards spiders: sensitivity to treatment and predictive value for generalization of treatment effects. Cognitive Therapy and Research.

Jones, M. K., \& Menzies, R. G. (2000). Danger expectancies, self-efficacy and insight in spider phobia. Behaviour Research and Therapy, 38, 585-600.

Jöreskog, K. G., \& Sörbom, D. (2002). LISREL 8.54. Chicago, Scientific Software International, Inc.

Kennedy, S. J., Rapee, R. M., \& Mazurski, E .J. (1997). Covariation bias for phylogenetic versus ontogenetic fearrelevant stimuli. Behaviour Research and Therapy, 35, 415-422.

Kleinknecht, R. A. (1993). Rapid Treatment of Blood and Injection Phobias with Eye Movement Desensitization. Journal of Behavioral Therapy and Experimental Psychiatry, 24, 211-217.

Kleinknecht, R. A., Kleinknecht, E. E., Sawchuk, C. N., Lee, T. C., \& Lohr, J. (1999). The Medical Fear Survey: psychometric properties. The Behavior Therapist, 22, 109-119.

Kleinknecht, R. A., Thorndike, R. M., \& Walls, M. M. (1996). Factorial dimensions and correlates of blood, injury, injection and related medical fears: cross-validation of the Medical Fear Survey. Behaviour Research and Therapy, 34, 323-331.

Klieger, D. M., \& Siejak, K. K. (1997). Disgust as the source of false positive effects in the measurement of ophidiophobia. Journal of Psychology, 131, 371-382.

Kline, R. B. (1998). Principles and practice of structural equation modeling. New York: Guilford.

Kline, K. P., Ginsburg, G. P., Johnston, J. R. (1998). T-wave amplitude: relationships to phasic RSA and heart period changes. International Journal of Psychophysiology, 29, 291-301.

Klorman, R., Weerts, T. C., Hastings, J. E., Melamed, B. G., \& Lang, P. J. (1974). Psychometric descriptions of some specific fear questionnaires. Behavior Therapy, 5, 401-422.

Koch, M. D., O'Neill, K., Sawchuk, C. N. \& Connolly, K. (2002). Domain-specific and generalized disgust sensitivity in blood-injection phobia: The application of behavioral approach/avoidance tasks. Journal of Anxiety Disorders, 16, 511-527.

Lang, P. J. (1979). A bio-informational theory of emotional imagery. Psychophysiology, 16, 495-512.

Lang, P. J., Bradley, M. M., \& Cuthbert, B. N. (1990). Emotion, attention, and the startle reflex. Psychological Review, 97, 377-395.

Lang, P. J., Bradley, M. M., \& Cuthbert, B. N. (1999). International affective picture system (IAPS). NIHMH centre for the study of emotion and attention, University of Florida, Gainesville, FL.

Lelliott, P., McNamee, G., \& Marks, I. (1991). Features of agora-, social, and related phobias and validation of the diagnoses. Journal of Anxiety Disorders, 5, 313-322.

Levenson, R.W. (1992). Autonomic nervous system differences among emotions. Psychological Science, 3, 23-27.

Lipsitz, J. D., Fyer, A.J., Paterniti, A., \& Klein, D. F. (2001). Emetophobia: preliminary results of an internet survey. Depression and Anxiety, 14, 149-152.

Lumley, M. A., \& Melamed, B. G. (1992). Blood phobics and nonphobics: Psychological differences and affect during exposure. Behaviour Research and Therapy, 30, 425-434

Lydiard, R. B., Laraia, M. T., Howell, E. F., \& Ballenger, J. C. (1986). Can panic disorder present as irritable bowel syndrome? Journal of Clinical Psychiatry, 47, 470-472.

MacLeod, C., Rutherford, E., Campbell, L., Ebsworthy, G., \& Holker, L. (2002). Selective attention and emotional vulnerability: assessing the causal basis of their association through the experimental manipulation of attentional bias. Journal of Abnormal Psychology, 111, 107-123. 


\section{REFERENCES}

Mancini, F., Gragnani, A., \& D'Olimpio, F. (2001). The connection between disgust and obsessions and compulsions in a non-clinical sample. Personality and Individual Differences, 31, 1173-1180.

Marks, L. M. (1987). Fears, phobias, and rituals. New York: Oxford University Press.

Marks, I. M., \& Mathews, A. M. (1979). Brief standard self-rating for phobic patients. Behaviour Research and Therapy, 17, 263-267.

Massop, M. (2005). Misselijk van angst; leven met een overgeeffobie. Antwerpen: Houtekiet.

Matchett, G., \& Davey, G. C. L. (1991). A test of disease-avoidance model of animal phobias. Behaviour Research and Therapy, 29, 91-94.

Mathews, A., \& Mackintosh, B. (2000). Induced emotional interpretation bias and anxiety. Journal of Abnormal Psychology, 109, 602-615.

Mathews, A., \& MacLeod, C. (2002). Induced processing biases have causal effects on anxiety. Cognition \& Emotion, $16,331-354$.

McDougall, S., Widdop, R., \& Lawrence, A. (2005). Central autonomic integration of psychological stressors: Focus on cardiovascular modulation. Autonomic Neuroscience, 123, 1-11.

McNally, R. J. (2002). Disgust has arrived. Journal of Anxiety Disorders, 16, 561-566.

McNally, R. J., \& Heatherton, T. F. (1993). Are covariation biases attributable to a priori expectancy biases? Behaviour Research and Therapy, 31, 653-658.

Menzel, P., \& D'Aluisio, F. (1998). Man eating bugs; the art and science of eating insects. Ten Speed Press: Berkely.

Merckelbach, H., de Jong, P. J., Arntz, A., \& Schouten, E. (1993). The role of evaluative learning and disgust sensitivity in the etiology and treatment of spider phobia. Advances in Behaviour Research and Therapy, 15, 243255.

Merckelbach, H., Muris, P., de Jong, P. J., \& de Jongh, A. (1999). Disgust sensitivity, blood-injection-injury fear, and dental anxiety. Clinical Psychology and Psychotherapy, 6, 279-285.

Miller, W. I. (1997). The anatomy of disgust. Harvard University Press, Cambridge.

de Montaigne, M., (1995). Four essays. Penguin Books, New York.

Moran, D. J., \& O'Brien, R. M. (2005). Competence imagery: a case study treating emetophobia. Psychological Reports, 96, 635-636.

Mulkens, S. A. N., de Jong, P. J., \& Merckelbach, H. (1996). Disgust and spider phobia. Journal of Abnormal Psychology, 105, 464-468.

Muris, P., \& Merckelbach, H. (1996). A comparison of two spider fear questionnaires. Journal of Behavioral Therapy and Experimental Psychiatry, 27, 241-244.

Muris, P., Merckelbach, H., Nederkoorn, S., Rassin, E., Candel, I., \& Horselenberg, R. (2000). Disgust and psychopathological symptoms in a non clinical sample. Personality and Individual Differences, 29, 1163-1167.

Muris, P., Merckelbach, H., Schmidt, H., \& Tierney, S. (1999). Disgust sensitivity, trait anxiety and anxiety disorders symptoms in normal children. Behaviour Research and Therapy, 37, 953-961.

Nederkoorn, C., Smulders, F. T. Y. \& Jansen, A. (1999). Recording of swallowing events using electromyography as a non-invasive measurement of salivation. Appetite, 33, 361-369.

Norris, M. B., Noble, A. C., \& Pangborn, R. M. (1984). Human saliva and taste responses to acids varying in anions, titratable acidity, and pH. Physiology and Behavior, 32, 237-244.

Olatunji, B. O. (2006). Evaluative learning and emotional responding to fearful and disgusting stimuli in spider phobia. Journal of Anxiety Disorders, 20, 858-876.

Olatunji, B. O., Arrindell, W. A., \& Lohr, J. M. (2005). Can the sex differences in disgust sensitivity account for the sex differences in blood-injection-injury fears? Personality and Individual Differences, 39, 61-71.

Olatunji, B. O., Cisler, J. M., Deacon, B. J., Connolly, K., \& Lohr, J. M. (2007). The Disgust Propensity and Sensitivity Scale-Revised: Psychometric properties and specificity in relation to anxiety disorder symptoms. Journal of Anxiety Disorders, 21, 918-930.

Olatunji, B. O., \& Deacon, B. J. (2008). Specificity of disgust sensitivity in the prediction of fear and disgust responding to a brief spider exposure. Journal of Anxiety Disorders, 22, 328-336.

Olatunji, B. O., Lohr, J. M., Sawchuk, C. N., \& Tolin, D. F. (2007). Multimodal assessment of disgust in contamination-related obsessive-compulsive disorder. Behaviour Research and Therapy, 45, 263-276. 
Olatunji, B. O., \& McKay, D. (2007). Disgust and psychiatric illness: Have we remembered? British Journal of Psychiatry, 190, 457-459.

Olatunji, B. O., \& Sawchuk, C. N. (2005). Disgust: characteristic features, social manifestations, and clinical implications. Journal of Social and Clinical Psychology, 24, 932-962.

Olatunji, B. O., Sawchuk, C. N., Arrindell, W. A., \& Lohr, J. M. (2005). Disgust sensitivity as a mediator of the sex differences in contamination fears. Personality and Individual Differences, 38, 713-722.

Olatunji, B. O., Sawchuk, C. N., de Jong, P. J., \& Lohr, J. M. (2006). The structural relation between disgust sensitivity and blood-injection-injury fears: A cross-cultural comparison of US and Dutch data. Journal of Behavior Therapy and Experimental Psychiatry, 37, 16-29.

Olatunji, B. O., Sawchuk, C. N., de Jong, P. J., \& Lohr, J. M. (2007). Disgust sensitivity in anxiety disorder symptoms: Factor structure and psychometric properties of the Disgust Emotion Scale. Journal of Psychopathology and Behavioral Assessment, 19, 281-297.

Olatunji, B. O., Sawchuk, C. N., Lohr, J. M, \& de Jong, P. J. (2004). Disgust domains in the prediction of contamination fear. Behaviour Research and Therapy, 42, 93-104.

Olatunji, B. O., Smits, J. A., Connolly, K. M., Willems, J., \& Lohr, J. M. (2007). Examination of the rate of decline in fear and disgust during exposure to threat-relevant stimuli in blood-injection-injury phobia. Journal of Anxiety Disorders, 21, 445-455.

Olatunji, B. O., Williams, N. L., Lohr, J. M., \& Sawchuk, C. N. (2005). The structure of disgust: domain specificity in relation to contamination ideation and excessive washing. Behaviour Research and Therapy, 43, 1069-1086.

Olatunji, B. O., Williams, N. L., Tolin, D. F., Sawchuk, C. N., Abramowitz, J. S., Lohr, J. M., \& Elwood, L. (2007). The Disgust Scale: Item analysis, factor structure, and suggestions for refinement. Psychological Assessment, 19, 281-297.

Öhman, A., Dimberg, U., \& Öst, L.-G. (1985). Animal and social phobias: biological constraints on learned fear responses. In S. Reiss \& R.R. Bootzin (Eds). Theoretical issues in behavior therapy (pp. 123-175). New York: Academic Press.

Öst, L-G. (1989). One-session treatment for specific phobias. Behaviour Research and Therapy, 27, 1-7.

Öst, L-G., Sterner, U., \& Lindahl, I.-L. (1984). Physiological responses in blood phobics. Behaviour Research and Therapy, 22, 109-117.

van Overveld, M., de Jong, P. J., \& Peters, M. L. (2006). Differential UCS-expectancy bias in spider fearful individuals: Evidence toward an assocation between spiders and disgust-relevant outcomes. Journal of Behavior Therapy and Experimental Psychiatry, 37, 60-72.

van Overveld, W.J.M., de Jong, P.J., Peters, M.L. (in press). Digestive and cardiovascular responses to core and animal-reminder disgust. Biological Psychology.

van Overveld, M., de Jong, P. J., Peters, M. L., van Hout, W. J. P. J., \& Bouman, T. K. (2008). An internet-based study on the relation between disgust sensitivity and emetophobia. Journal of Anxiety Disorders, 22, 524-531.

van Overveld, W. J. M., de Jong, P. J., Peters, M. L., Cavanagh, K., \& Davey, G. C. L. (2006). Disgust propensity and disgust sensitivity: separate constructs that are differentially related to specific fears. Personality and Individual Differences, 41, 1241-1252.

Page, A. C. (1994). Blood-injury phobia. Clinical Psychology Review, 14, 443-461.

Page, A. C. (2003). The role of faintness elicited by blood and injection stimuli. Journal of Anxiety Disorders, 17, 4558.

Palomba, D., Sarlo, M., Angrilli, A., Mini, A. \& Stegagno, L. (2000). Cardiac responses associated with affective processing of unpleasant film stimuli. International Journal of Psychophysiology, 36, 45-57.

Pauli, P., Montoya, P., \& Martz, G-E (1996). Covariation Bias in Panic-Prone Individuals. Journal of Abnormal Psychology, 105, 658-662.

Pauli, P., Montoya, P., \& Martz, G-E (2001). On-line and a posteriori covariation estimates in panic-prone individuals: effects of a high contingency of shocks following fear-irrelevant stimuli. Cognitive Therapy and Research, 25, 103116.

Pauli, P., Wiedemann, G., \& Montoya, P. (1998). Covariation bias in flight phobics. Journal of Anxiety Disorders, 12, 555-565. 


\section{REFERENCES}

Philips, H. C. (1985). Return of fear in the treatment of a fear of vomiting. Behaviour Research and Therapy, 23, 4553.

Phillips, M. L., Senior, C., Fahy, T., \& David, A. S. (1998). Disgust - the forgotten emotion of psychiatry. British Journal of Psychiatry, 172, 373-375.

Pollard, C. A., Tait, R. C., Meldrum, D., Dubinsky, I. H., \& Gall, J. S. (1996). Agoraphobia without panic: case illustrations of an overlooked syndrome. The Journal of Nervous and Mental Diseases, 184, 61-62.

Power, M. J., \& Dalgleish, T. (1997). Cognition and emotion: From order to disorder. Psychology Press, Hove.

Prkachin, K. M., Williams-Avery, R. M., Zwaal, C., \& Mills, D. E. (1999). Cardiovascular changes during induced emotion: an application of Lang's theory of emotional imagery. Journal of Psychosomatic Research, 47, 255-267.

Proctor, G. B., \& Carpenter, G. H. (2007). Regulation of salivary gland function by autonomic nerves. Autonomic Neuroscience: Basis and Clinical, 133, 3-18.

Pury, C. L. S., \& Mineka, S. (1997). Covariation bias for blood-injury stimuli and aversive outcomes. Behaviour Research and Therapy, 35, 35-47.

Rau, H. (1991). Responses of the T-wave amplitude as a function of active and passive tasks and beta-adrenergic blockade. Psychophysiology, 28, 231-239.

Reiss, S. (1991). Expectancy theory of fear, anxiety and panic. Clinical Psychology Review, 11, 141-153.

Reiss, S., Peterson, R. A., Gursky, D. M., \& McNally, R. J. (1986). Anxiety sensitivity, anxiety frequency, and the prediction of fearfulness. Behaviour Research and Therapy, 24, 1-8.

Ritow, J. K. (1979). Brief treatment of a vomiting phobia. The American Journal of Clinical Hypnosis, 21, 293-494.

Ritz, T., Wilhelm, F. H., Gerlach, A. L., Kullowatz, A., \& Roth, W. T. (2005). End-tidal $\mathrm{pCO}_{2}$ in blood phobics during viewing of emotion- and disease-related films. Psychosomatic Medicine, 66, 661-668.

Roberts, M. J. (1995). Spiders of Britain \& Northern Europe. London: HarperCollins Publishers.

Rohrmann, S., \& Hopp, H. (2008). Cardiovascular indicators of disgust. International Journal of Psychophysiology, 68, 201-208.

Rozin, P., \& Fallon, A. E. (1987). A perspective on disgust. Psychological Review, 94, $23-41$.

Rozin, P., Fallon, A. E., \& Mandell, R. (1984). Family resemblance in attitudes to food. Developmental Psychology, 20, 309-314.

Rozin, P., Haidt, J., McCauley, C. R. (1999). Disgust: the body and soul emotion. In Dalgleish, T., \& Power, M. (1999). Handbook of Cognition and Emotion. New York: John Wiley \& Sons Ltd.

Rozin, P., Haidt, J., \& McCauley, C. R. (2000). Disgust. In Lewis, M. \& Haviland Jones, J. M. (Ed.), Handbook of Emotions, $2^{\text {nd }}$ edition (637-652). New York: Guilford Press.

Rozin, P., Haidt, J., McCauley, C., Dunlop, L., \& Ashmore, M. (1999). Individual differences in disgust sensitivity: comparisons and evaluations of paper-and-pencil versus behavioral measures. Journal of Research in Personality, 33, 330-351.

Rozin, P., Markwith, M., \& McCauley, C. (1994). Sensitivity to indirect contact with other persons: AIDS aversion as a composite of aversion to strangers, infection, moral taint, and misfortune. Journal of Abnormal Psychology, 103, 495-504.

Rozin, P., Nemeroff, C., Horowitz, M., Gordon, B., \& Voet, W. (1995). The borders of the self: contamination sensitivity and potency of the body apertures and other body parts. Journal of Research in Personality, 29, 318340.

Russel, J. F. (1979). Tarantism. Medical History, 23, 404-425.

Sanderman, R., Arrindell, W. A., Ranchor, A. V., Eysenck, H. J., \& Eysenck, S. B. G (1995). Het meten van persoonlijkheidskenmerken met de Eysenck Personality Questionnaire (EPQ): een handleiding. Noordelijk Centrum voor Gezondheidsvraagstukken, Groningen.

Sarlo, M., Palomba, D., Angrilli, A., \& Stegagno, L. (2002). Blood phobia and spider phobia: two specific phobias with different autonomic cardiac modulations. Biological Psychology, 60, 91-108.

Sartre, J. P. (1976). Walging. Uitgeverij de Arbeiderspers, Amsterdam.

Sawchuk, C. N., Lohr, J. M., Lee, T. C., \& Tolin, D. F. (1999). Exposure to disgust-evoking imagery and information processing biases in blood-injection-injury phobia. Behaviour Research and Therapy, 37, 249-257.

Sawchuk, C. N., Lohr, J. M., Tolin, D. F., Lee, T. C., \& Kleinknecht, R. A. (2000). Disgust sensitivity and contamination fears in spider and blood-injection-injury phobias. Behaviour Research and Therapy, 38, 753-762. 
Sawchuk, C. N., Lohr, J. M., Westendorf, D. H., Meunier, S. A., \& Tolin, D. F. (2002). Emotional responding to fearful and disgusting stimuli in specific phobia. Behaviour Research and Therapy, 40, 1031-1046.

Schafer, J. L, \& Graham, J. W. (2002). Missing data: our view of the state of the art. Psychological Methods, 7, 147177.

Schienle, A., Schäfer, A., Walter, B., Stark, R., \& Vaitl, D. (2005). Elevated disgust sensitivity in blood phobia. Cognition \& Emotion, 19, 1229-1241.

Schienle, A., Stark, R., \& Vaitl (2001). Evaluative conditioning: A possible explanation for the acquisition of disgust responses? Learning and Motivation, 32, 65-83.

Schienle, A., Stark, R., Walter, B, \& Vaitl, D. (2003). The connection between disgust sensitivity and blood-related fears, faintness symptoms and obsessive-compulsiveness in a non-clinical sample. Anxiety, Stress and Coping, 16, 185-193.

Segerstrom, S. C., \& Miller, G. E. (2004). Psychological stress and the human immune system: a meta-analytical study of 30 years of inquiry. Psychological Bulletin, 130, 601-630.

Shapiro, D., Jamner, L. D., Lane, J. D., Light, K. C., Mytrek, M. Sawada, Y., \& Steptoe, A. (1996). Blood pressure guidelines. Psychophysiology, 33, 1-12.

Snell, W. E., Jr., Gum, S., Shuck, R. L., Mosley, J. A., \& Hite, T. L. (1995). The Clinical Anger Scale: Preliminary reliability and validity. Journal of Clinical Psychology, 51, 215-226.

van Strien, T., Frijters, J. E. R., Bergeres, G. P. A., \& Defares, P. B. (1986). Nederlandse vragenlijst voor eetgedrag. Swets, \& Zeitlinger, Lisse.

Szymanski, J., \& O'Donohue, W. (1995). Fear of spiders questionnaire. Journal of Behavior Therapy and Experimental Psychiatry, 26, 31-34.

Task Force of the European Society of Cardiology and the North American Society of Pacing and Electrophysiology (1996). Heart rate variability: standards of measurement, physiological interpretation and clinical use. Circulation, 293, 1043-65.

Taylor, S. (1993). The structure of fundamental fears. Journal of Behavior Therapy and Experimental Psychiatry, 24, 289-299.

Teachman, B. A., \& Woody, S. R. (2003). Automatic processing in spider phobia: implicit fear associations over the course of treatment. Journal of Abnormal Psychology, 112, 100-109.

Thordarson, D. S., Radomsky, A. S., Rachman, S., Shafran, R., Sawchuk, C. N., \& Hakstian, A. R. (2004). The Vancouver Obsessional Compulsive Inventory (VOCI). Behaviour Research and Therapy, 42, 1289-1314.

Thorpe, S. J., Patel, S. P., \& Simonds, L. M. (2003). The relationship between disgust sensitivity, anxiety and obsessions. Behaviour Research and Therapy, 41, 1397-1409.

Thorpe, S., \& Salkovskis, P. M. (1998). Studies on the role of disgust in the acquisition and maintenance of specific phobias. Behaviour Research and Therapy, 36, 877-893.

Tolin, D. F., Lohr, J. M., Sawchuk, C. N., \& Lee, T. C. (1997). Disgust and disgust sensitivity in blood-injection-injury and spider phobia. Behaviour Research and Therapy, 35, 949-953.

Tomarken, A. J., Mineka, S., \& Cook, M. (1989). Fear-relevant selective associations and covariation bias. Journal of Abnormal Psychology, 98, 381-394.

Tomarken, A. J., Sutton, S. K., \& Mineka, S. (1995). Fear-relevant illusory correlations: what types of associations promote judgmental bias? Journal of Abnormal Psychology, 104, 312-326.

Troop, N. A., Murphy, F., Bramon, E., \& Treasure, J. L. (2000). Disgust sensitivity in eating disorders: a preliminary investigation. International Journal of Eating Disorders, 27, 446-451.

Troop, N. A., Treasure, J. L., \& Serpell, L. (2002). A further exploration of disgust in eating disorders. European Eating Disorders Review, 10, 218-226.

Tsao, S.D. \& McKay, D. (2004). Behavioral avoidance tests and disgust in contamination fears: distinctions from trait anxiety. Behaviour Research and Therapy, 42, 207-216.

Veale, D., \& Lambrou, C. (2006). The psychopathology of vomit phobia. Behavioural and Cognitive Psychotherapy, 34, $139-150$.

Vetter, R. S., Cushing, P. E., Crawford, R. L \& Royce, L. A. (2003). Diagnoses of brown recluse spider bites (loxoscelism) greatly outnumber actual verifications of the spider in four western American states. Toxicon, 42, 413-418. 


\section{REFERENCES}

Vögele, C., Coles, J., Wardle, J., \& Steptoe, A. (2003). Psychophysiologic effects of applied tension on the emotional fainting response to blood and injury. Behaviour Research and Therapy, 41, 139-155.

Vrana, S. R. (1993). The psychophysiology of disgust: differentiating negative emotional contexts with facial EMG. Psychophysiology, 30, 279-286.

Vrana, S. R., Spence, E.L., \& Lang, P. J. (1988). The startle probe response: A new measure of emotion? Journal of Abnormal Psychology, 97, 487-491.

Walls, M. M., \& Kleinknecht, R. A. (1996). Disgust factors as predictors of blood-injury fear and fainting. Paper presented at the annual meeting of the Western Psychological Association, San Jose, CA.

Wardle, J. (1987). Eating style: A validation study of the Dutch Eating Behaviour Questionnaire in normal subjects and women with eating disorders. Journal of Psychosomatic Research, 31, 161-169.

Ware, J., Jain, K., Burgess, I. \& Davey, G. C. L. (1994). Disease-avoidance model: factor analysis of common animal fears. Behaviour Research and Therapy, 32, 57-63.

Webb, K., \& Davey, G. C. L. (1992). Disgust sensitivity and fear of animals: effect of exposure to violent or repulsive material. Anxiety, Stress and Coping, 5, 329-335.

Wenzel, A., \& Holt, S. J. (2003). Validation of the Multidimensional Blood-injury Phobia Inventory: evidence for a unitary construct. Journal of Psychopathology and Behavioral Assessment, 25, 203-211.

Whittal, M. L., \& Goetsch, V. L. (1997). The impact of panic expectancy and social demand on agoraphobic avoidance. Behaviour Research and Therapy, 35, 813-821.

White, K. S., \& Sher, K. J. (1977). Saliva: A review and experimental investigation of major techniques. Psychophysiology, 14, 203-212.

Wiedemann, G., Pauli, P., \& Dengler, W. (2001). A priori expectancy bias in patients with panic disorder. Journal of Anxiety Disorders, 15, 401-412.

Wolpe, L., \& Lang, P. J. (1964). A fear survey schedule for use in behaviour therapy, Behaviour Research and Therapy, 2, 27-30.

Woody, S. R., McLean, C., \& Klassen, T. (2005). Disgust as a motivator of avoidance of spiders. Journal of Anxiety disorders, 19, 461-475.

Woody, S. R., \& Teachman, B. A. (2000). Intersection of disgust and fear: Normative and pathological views. Clinical Psychology: Science and Practice, 7, 291-311.

Woody, S. R., \& Tolin, D. F. (2002). The relationship between disgust sensitivity and avoidant behavior: studies of clinical and nonclinical samples. Journal of Anxiety Disorders, 16, 543-559

Yartz, A. R., \& Hawk Jr, L. W., (2002). Addressing the specificity of affective startle modulation: fear versus disgust. Biological Psychology, 59, 55-68.

Zung, W. W. K. (1965). A self-rating depression scale. Archives of General Psychiatry, 12, 63-70. 


\section{Biography}

'Spider Pig,

Spider pig,

Does whatever a spider pig does'

Homer Simpson 
BIOGRAPHY 


\section{Biography}

Mark van Overveld was born on july 13th 1975 in Oudenbosch, Netherlands. In 1994, he obtained his VWO diploma at the Thomas More College in Oudenbosch. Next, he studied both Health Sciences (with a major in Mental Health Sciences) and Biological Psychology at Maastricht University. In 2002, he received his master's degree for both studies and started working on the PhD-project 'Disgust in specific phobias' at the Department of Clinical Psychological Science (former Department of Medical, Clinical, and Experimental Psychology). The studies that were conducted during this PhD-period are presented in this dissertation. 


\section{Publications}

'Forget Everything And Remember, Find Earth And Reap.' Ian Brown 


\section{PUBLICATIONS}

\section{Publications}

de Jong, P. J., van Overveld, M., Weijmar-Schultz, W., Peters, M.L., \& Buwalda, F.M. (in press). Disgust and contamination sensitivity in vaginismus and dyspareunia. Archives of Sexual Behavior.

van Overveld, M., de Jong, P. J., \& Peters, M. L. (2006). Differential UCS-expectancy bias in spider fearful individuals: Evidence toward an assocation between spiders and disgust-relevant outcomes. Journal of Behavior Therapy and Experimental Psychiatry, 37, 60-72.

van Overveld, M., de Jong, P. J., Peters, M. L., van Hout, W. J. P. J., \& Bouman, T. K. (2008). An internet-based study on the relation between disgust sensitivity and emetophobia. Journal of Anxiety Disorders, 22, 524-531.

van Overveld, W.J.M., de Jong, P.J., \& Peters, M.L. (2004). Walging: van emotie tot angststoornis. Nederlands Tijdschrift voor de Psychologie. 59, 33-44.

van Overveld, W. J. M., de Jong, P. J., Peters, M. L., Cavanagh, K., \& Davey, G. C. L. (2006). Disgust propensity and disgust sensitivity: separate constructs that are differentially related to specific fears. Personality and Individual Differences, 41, 1241-1252.

van Overveld, W. J. M., de Jong, P. J., \& Peters, M. L. (2008). Disgust propensity and disgust sensitivity are differentially associated with psychopathological symptoms. Journal of Affective Disorders, 107, p S120. (poster announcement for the conference of the International Society for Affective Disorders, Kaapstad, 2008).

\section{Submitted manuscripts}

van Overveld, W.J.M., de Jong, P.J., Huijding, J., \& Peters, M.L. (2007). Contamination and harm relevant UCSexpectancy bias in spider phobic individuals: influence of treatment and its relationship with the return of fear.

van Overveld, W.J.M., de Jong, P.J., Peters, M.L. (2007). Digestive and cardiovascular responses to core and animalreminder disgust.

van Overveld, W.J.M., de Jong, P.J., \& Peters, M.L. (2007). Disgust propensity and disgust sensitivity are differentially associated with psychopathological symptoms.

van Overveld, W.J.M., de Jong, P.J., \& Peters, M.L. (2007). The Disgust Propensity and Sensitivity Scale - Revised: its predictive value for avoidance behavior and physiological responsivity.

van Overveld, W.J.M., de Jong, P.J., \& Peters, M.L. (2007). The Disgust Scale-R: $A$ valid and reliable index to investigate separate disgust domains?

\section{Presentations}

van Overveld, W.J.M., de Jong, P.J., \& Peters, M.L. (2003). Disgust / disease versus harm-related outcome expectancies in spider phobia. Poster presentation at the $37^{\text {th }}$ annual conference of the Association for Advancement of Behavior Therapy, Boston, USA.

van Overveld, W.J.M., de Jong, P.J., \& Peters, M.L. (2004). The Disgust Propensity and Sensitivity Scale: a study on psychometric qualities. Poster presentation at the $38^{\text {th }}$ jaarlijkse congres van de Association for Advancement of Behavior Therapy, New Orleans, USA.

van Overveld, W.J.M., de Jong, P.J., \& Peters, M.L. (2005). Startle potentiation and facial EMG during disgust and fear stimuli in high and low spider fearful individuals. Poster presentation at the second Meeting on Experimental Psychopathology, University of Leuven, Belgium.

van Overveld, W.J.M., de Jong, P.J., Huijding, J., \& Peters, M.L. (2005). Differential UCS expectancy bias in spider phobic individuals: influence of treatment and its relationship with the return of fear. Poster presentation at the $35^{\text {th }}$ annual conference of the European Association for Behavioural and Cognitive Therapies, Thessaloniki, Greece. 
van Overveld, W.J.M., de Jong, P.J., Huijding, J., \& Peters, M.L. (2005). Differential UCS expectancy bias in spider phobic individuals: influence of treatment and its relationship with the return of fear. Poster presentation at the $39^{\text {th }}$ annual conference of the Association for Behavioral and Cognitive Therapies, Washington DC, USA.

van Overveld, W.J.M. (2006). Why do insects make our skin crawl? Presentation for Studium Generale, University of Wageningen.

van Overveld, W.J.M., de Jong, P.J., Peters, M.L., van Hout, W.J.P.J., \& Bouman, T.K. (2006). Emetophobic individuals are characterized by elevated levels of disgust propensity and sensitivity. Poster presentation at the $36^{\text {th }}$ annual conference of the European Association for Behavioural and Cognitive Therapies, Paris, France.

van Overveld, W.J.M (2007). Disgust and clinical disorders. Presentation at the Departement of Psychology, University of Jena, Jena, Germany.

van Overveld, W.J.M., de Jong, P.J., \& Peters, M.L. (2007). Disgust and fear-related UCS expectancy bias in blood fearful individuals. Poster presentation at the World Congress for Behavioral and Cognitive Therapies in Barcelona, Spain.

van Overveld, W.J.M (2007). The Disgust Propensity and Sensitivity Scale: a valuable addition to research? Presentation at the World Congress for Behavioral and Cognitive Therapies in Barcelona, Spain.

van Overveld, W.J.M., de Jong, P.J., \& Peters, M.L. (2007). Disgust and fear-related UCS expectancy bias in blood fearful individuals. Poster presentation at the $2^{\text {nd }}$ annual EPP-day in Utrecht.

van Overveld, W.J.M., de Jong, P.J., \& Peters, M.L. (2007). Disgust and fear-related UCS expectancy bias in blood fearful individuals. Presentation at the $2^{\text {nd }}$ annual EPP-dag in Utrecht.

van Overveld, W.J.M., de Jong, P.J., \& Peters, M.L. (2008). Disgust propensity and disgust sensitivity are differentially associated with psychopathological symptoms. Poster presentation at the $4^{\text {th }}$ biannual conference of the International Society for Affective Disorders in Cape Town, South Africa. 


\section{Dankwoord}

'Thanks to everyone for everything you've done But now it's time to go.'

Supergrass 


\section{DANKWOORD}

In veel opzichten is het schrijven van een dissertatie een zware exercitie. Vergeleken met het neerpennen van mijn scriptie, dat zich liet omschrijven als een aangenaam fietstochtje over een asfaltweg door fraai Limburgs heuvellandschap, bleek mijn proefschrift bij tijd en wijle een uitputtingsslag langs robuuste grindweggetjes kris-krassend door de Himalayas. En ik ben al geen fietser. Het moge dan ook geen verbazing wekken dat het bereiken van het einde van deze dirtroad dan ook voor een groot deel te wijten is aan een paar speciale mensen in mijn omgeving.

Allereerst bleken mijn beide begeleiders wél voortvarende fietsers. Ik kan niet anders dan concluderen dat ik werkelijk bevoorrecht ben geweest te hebben mogen werken met deze twee bijzondere wetenschappers. Madelon, enige jaren geleden strompelde ik je kamer binnen omdat ik op zoek was naar een afstudeerstage met 'iets biologisch' en jij de blokcoördinator was van één van de leukste blokken uit het curriculum naar psychofysiologie. Je wist me over te halen iets compleet anders te doen, en zo zette ik mijn eerste stapjes in het (pijn-)onderzoek. Het werd een relatief moeizaam onderzoek dat onder jouw begeleiding echter zo nimmer heeft aangevoeld. En ook de afgelopen 'walgelijke' jaren vlogen voorbij. Hoe druk je het zelf ook had, er was altijd tijd voor een snelle vraag of even af te spreken als ik ergens niet mee vooruitkwam, of het nu ging over bespreken van weer een afwijzing, een komende sollicitatie, of even na te bespreken hoe een congres was verlopen. Dank voor de vele nuttige tips die je altijd voor me had en de erg plezierige open-deur policy. Ook de ruimte en het vertrouwen die ik regelmatig mocht genieten heb ik ten zeerste gewaardeerd.

Peter, hoewel na mijn start als aio je betrekkelijk snel verhuisde richting Groningen en dat walgelijke mannetje achterliet in de catacomben van DMKEP, heb ik het nooit ervaren dat je feitelijk enkele honderden kilometers van het project was verwijderd. Bedankt voor al je enorme toewijding, inzet en actieve meedenken. Of het nu ging over onbenullige vragen of ik een bepaald mailtje wel zo kon sturen naar iemand of het design van een nieuwe studie, je stond altijd klaar om me snel een antwoord te verschaffen via mail of telefoon. Zelfs op de meest vreemde tijdstippen kon ik nog rekenen op een volledig gecorrigeerd document vol rode strepen en gouden tips. Om nog maar te zwijgen van de stortvloed aan ideeën die het gemiddelde gesprek bij het koffiezetapparaat met jou oplevert.

Ik had me geen betere voorbeelden voor mijn vorming tot wetenschapper kunnen wensen dan jullie beiden, twee wetenschappers met een overweldigende passie voor het vak, die zowel publiceren als integriteit hoog in het vaandel hebben staan. Ik kan jullie niet genoeg bedanken voor de plezierige samenwerking van de afgelopen jaren. Bovendien werkt jullie beider enthousiasme over 'het onderzoek' zonder meer aanstekelijk. Dank, dank, dank...

Angela, je bent onbetwist de grote onmisbare spin zonder wie er geen DMK-web mogelijk zou zijn. Je bent niet alleen een fier bewaakster van de schatkist van het Departement, maar vooral ook iemand die de sociale cohesie van de vakgroep nauwlettend in het oog houdt waardoor we nog wel eens dankbaar in Thembi of Spa eindigen en onze collegas beter leren kennen. Een groot goed. Bedankt voor al je steun en goede zorgen in deze.

Behind every great man, there is a great statistician. Of zoiets was het meen ik. Erik, dank voor al je hulp en je bereidheid elk futiel of stom statistisch verzoekje mijnerzijds toe te lichten met een heldere verklaring. Elsa, bedankt voor je hulp rondom de bureaucratische rompslomp 
in de laatste fase. Rense, bedankt voor het lenen van een koelkast zonder welke één der studies vele malen walgelijker was geworden dan wenselijk. Charlie, Rosanne, en Theo, ook jullie bedankt voor alle hulp omtrent de studies in het lab en ontwerpen van de benodigde software ervoor. Er is geen crisis die jullie niet bezweren konden, al mijn pogingen ten spijt! Zeker ook dank aan Ruud en Jeroen van Datawyse wiens vele tips het einddocument werkelijk dissertatiewijs maakten, en Tristan voor de bijpassende en fraaie verpakking ervan.

Corine, Corine, Denise, Esther, Jeanine, Jill, Karoline, Linda, Linsey, Lotte, Maaike, Manon, Mattijn, Nicole, Roy, Ruud, Saskia, Tim en alle promovendi van DMKEP en CPS met wie ik heb mogen samenwerken of stoom afblazen, bedankt voor alle onvergetelijke ervaringen. Dankbaar denk ik aan het krabbelen van onderzoeksideetjes op bierviltjes na een kerstborrel met Jorg, quizen met de Belgische delegatie, ping-pongen in Soesterberg met Jeffrey, de vele promovendi-etentjes, de altijd gezellige maandelijkse promovendi-vergaderingen. Anneke, bedankt voor onze tijd samen op kamer 1.356. Bij jou op de kamer bleef ik mooi op de hoogte van alle ontwikkelingen en roddels binnen DMKEP en jouw enthousiaste houding was erg prettig als kamergenoot. Lea, ook jij bedankt voor een leuke tijd samen. De discussies die reikten van Word-perikelen tot de BeeGees en Lou Reed zal ik absoluut missen. Wie anders binnen DMKEP kent dat ene hitje van de prinses van Monaco als je er even niet opkomt?

Natuurlijk ook de mensen van de PhD-Academy. In 2006 besloten Saskia en Mariolina ProvUM, een welhaast begraven organisatie voor promovendi over de hele universiteit, een doorstart te gunnen. Al snel groeide het initiatief, waarin jullie mij vanaf de start ook betrokken ten behoeve van het sociale comité, uit tot een bloeiende organisatie met vertegenwoordigers op alle onderzoeksinstituten binnen de UM, die maandelijks goedbezochte en leuke evenementen organiseert. Van filmavonden tot discussies in de Salon, of gewoon een spetterend feest. Saskia, Mariolina, Gjalt-Yorn, Adrienne, Merel, Stephanie, Siu-Hing, Antoine, Sharon, Mirjam, en alle andere mensen van de PhD-academy, dank voor alle gezellige momenten.

Dank ook aan de verschillende scriptiestudenten van wie ik uiteindelijk minstens zoveel heb geleerd als vice versa. Met name Dieneke, Ernst, Franca, Ilona, Joëlle, Marthe, bedankt voor de onvergetelijke ervaringen in het lab, de persoonlijke verhalen en alle mooie momenten.

Tenslotte nog een woord aan enkele mensen van buiten de academische contreien die weliswaar niet vanuit hun professie maar wel ongezien kundig me door strubbelingen loodsten.

Maike en Wim, dank voor alle hulp op alle mogelijke vlakken. Hoewel ik vermoed dat jullie een grotere rol in het proces hadden gewenst, hebben jullie onbewust een grotere invloed op het proefschrift gehad dan jullie misschien beseffen. Bijvoorbeeld door altijd voor mij en Jacqueline klaar te staan wanneer we het nodig hebben. Ik zal jullie vast te weinig met lange verhalen over mijn werk vervelen, soms is er simpelweg ook niet veel te vertellen. Maar als het stil is, betekent dat niet dat jullie invloed absent is. Sterker nog, één van de grootste drijfveren voor het voltooien hiervan is nog immer de wetenschap dat juist jullie trots op dit bundeltje papiertjes zullen zijn. 


\section{DANKWOORD}

Maartje en Maddy, zonder twee excellente paranymfen zal de promotiedag in het honderd lopen. Gelukkig weet ik me gesteund met twee zusjes met een uitzonderlijk talent in zowel organisatorisch als sociaal opzicht, dus dank dat ik me daar geen zorgen over hoef te maken.

Wiebe, Jeanneke, BJ, Chris, Niels, Kristien, en alle andere vrienden die ik rijk ben, dank voor alle dierbare herinneringen en mooie vriendschappen zonder welke het leven kleurloos en saai zou zijn. Zonder jullie kundige afleidingsmanoeuvres komt er geen letter op papier. Anirion, Elthan, Myngwen, en Risa, het is fijn om zo af en toe even de Word-documenten terzijde te schuiven om gezamenlijk een avondje het dagelijkse leven compleet te vergeten. Whachawedu?

Zomotta, waar zou ik zijn zonder jou. Elke zondag sta je voor me klaar om de beslommeringen van de week van me af te werpen. Ik hoop nog lang onderdeel van dit uniek stukje muziekgeschiedenis te mogen zijn. Niets zo prachtig als het lab uitrennen, de auto in om uren later een vlammend optreden te verzorgen voor een uitverkochte Melkweg, en de volgende dag weer vrolijk te ploeteren aan een review. Op het hoogtepunt deden we dertig optredens in een jaar door heel Nederland naast het proefschrift, maar ik heb er elke seconde van genoten en nieuwe energie uit kunnen putten. Op naar de volgende plaat!

Last but not least, Jacqueline. Hoewel je zelf uiteindelijk het schrijven van het proefschrift moest staken, heb je altijd onverminderd in mij geloofd. Bovendien heb je me nimmer het gevoel gegeven dat ik me bezwaard zou moeten voelen omdat het mij wel lukte. Zoals ik enorme bewondering koester voor jouw vermogen om vrijwel meteen een nieuwe en succesvolle carrière in een volstrekt nieuwe richting te beginnen, bleef jij trots op mij en hebt me geholpen waar je kon. Er is geen pilotstudie waar je niet aan hebt meegedaan, geen artikel waar je me niet over hebt uitgehoord, er er was geen muzikale quote die zonder jouw kritisch meeluisteren in het proefschrift kwam. Alle walgelijke tests uit deze dissertatie heb je aan den lijve ondergaan, en uiteraard heb je me daarvoor met Kerst ook wel eens teruggepakt. Je glimlach en je humor zijn voor mij onmisbare elementen voor het slagen van alles wat ik onderneem. Relativerende woorden als 'Spiders are ugly creatures, but frogs are worse!' staan in mijn geheugen gegrifd. Daarom passeerde één typerende quote ongezien jouw ogen, van één van mijn favoriete artiesten die één van jouw favoriete artiesten covert, en die treffend parafraseert hoeveel je betekent voor mij.

'You could have it all. My empire of dirt'

Johnny Cash 